\title{
WNT Signaling in Cardiac and Vascular Disease
}

Sébastien Foulquier, Evangelos P. Daskalopoulos, Gentian Lluri, Kevin C. M. Hermans, Arjun Deb, and W. Matthijs Blankesteijn Department of Pharmacology and Toxicology, Cardiovascular Research Institute, Maastricht University, Maastricht, The Netherlands (S.F., K.C.M.H., W.M.B.); Recherche Cardiovasculaire (CARD), Institut de Recherche Expérimentale et Clinique (IREC), Université catholique de Louvain, Brussels, Belgium (E.P.D.); Department of Medicine, Division of Cardiology, David Geffen School of Medicine (G.L., A.D.); and Department of Molecular Cell and Developmental Biology, University of California at Los Angeles, Los Angeles, California (A.D.)

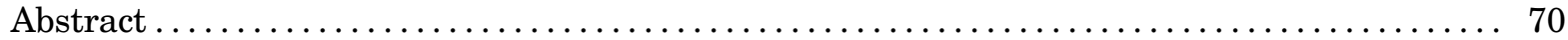

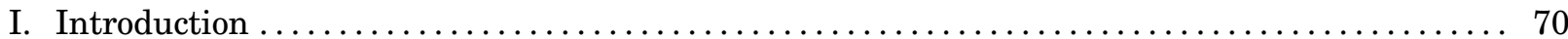

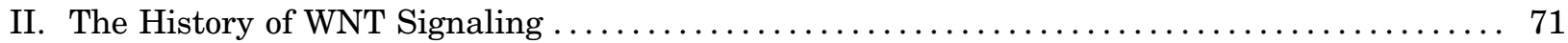

III. Components of the Receptor Complex .................................. 71

A. Synthesis and Posttranslational Modifications of WNT $\ldots \ldots \ldots \ldots \ldots \ldots \ldots \ldots \ldots \ldots \ldots \ldots$

B. Frizzled Proteins............................................. 73

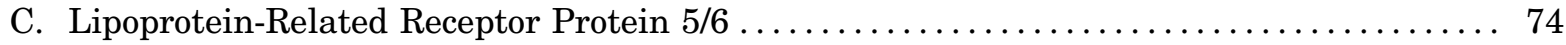

D. Receptor Tyrosine Kinase-like Orphan Receptor $1 / 2$ and Ryk................... 74

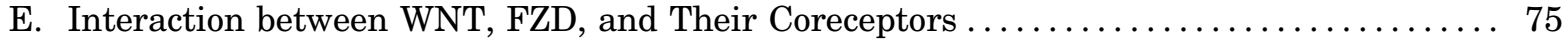

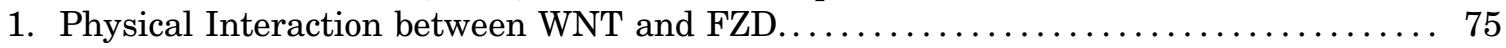

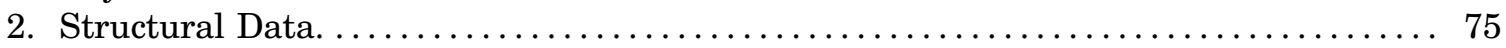

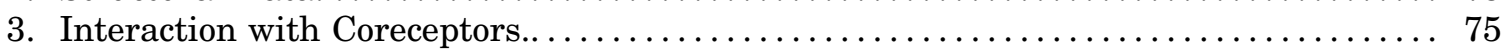

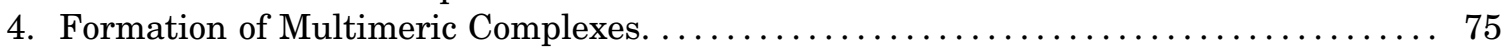

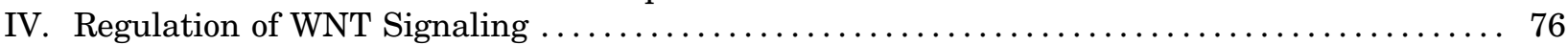

A. WNT/Notum/Tiki .............................................. 76

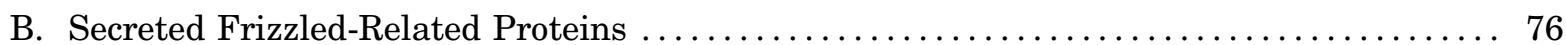

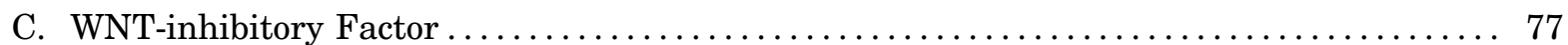

D. Leucine-rich Repeat-containing G Protein-Coupled Receptor 4/5/R-Spondin . . . . . . . . . 77

E. Dickkopf ................................................... 78

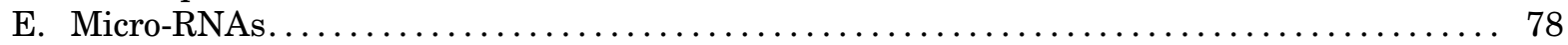

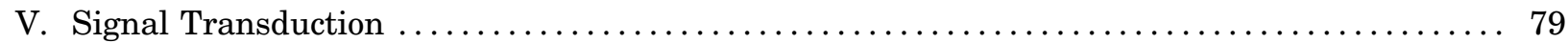

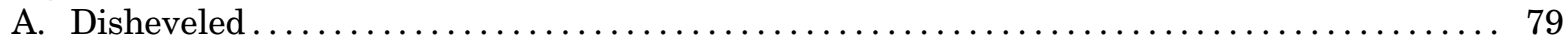

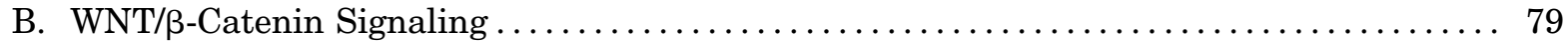

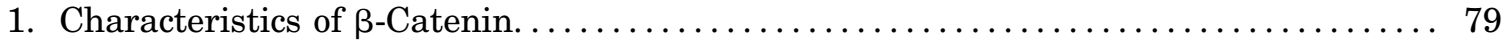

2. The $\beta$-Catenin Destruction Complex. .............................. 80

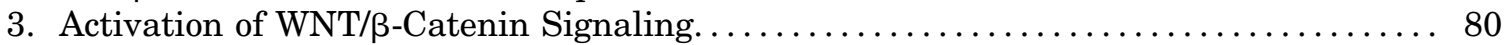

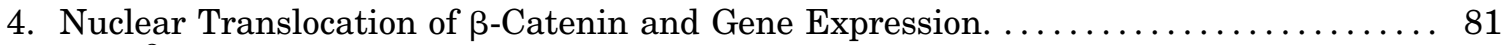

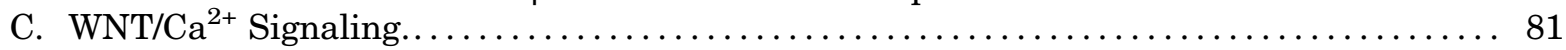

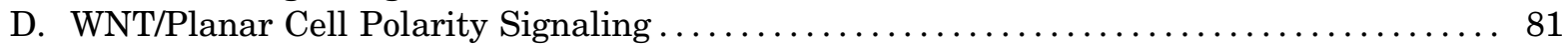

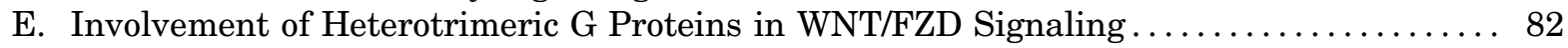

VI. WNT Signaling in Cardiac and Vascular Development $\ldots \ldots \ldots \ldots \ldots \ldots \ldots \ldots \ldots \ldots \ldots \ldots 83$

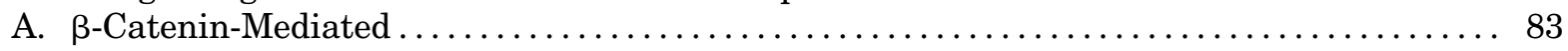

B. Involvement of Non- $\beta$-Catenin-Mediated WNT Signaling $\ldots \ldots \ldots \ldots \ldots \ldots \ldots \ldots \ldots \ldots$

C. Formation of Outflow Tract, Valves, and Conduction System ................... 84

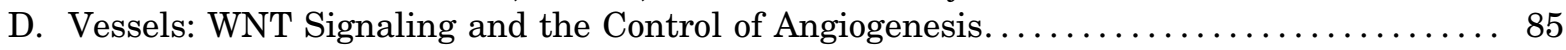

1. WNT Signaling Elements in Endothelial Cells .......................... 85

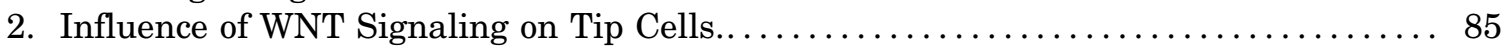

3. Influence of WNT Signaling on Stalk Cells.......................... 86

S.F. and E.P.D. contributed equally to the work.

Address correspondence to: W. Matthijs Blankesteijn, Department of Pharmacology and Toxicology, Cardiovascular Research Institute Maastricht, Maastricht University, 50 Universiteitssingel, 6229 ER Maastricht, The Netherlands. E-mail: wm.blankesteijn@maastrichtuniversity.nl

https://doi.org/10.1124/pr.117.013896. 
4. WNT Signaling and Stabilization and Specification of the Vascular Network. ..... 86

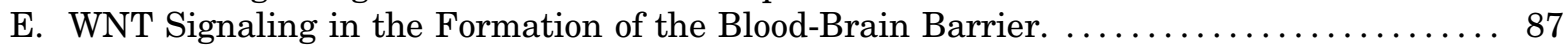

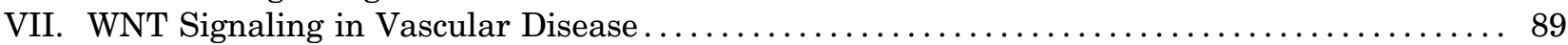

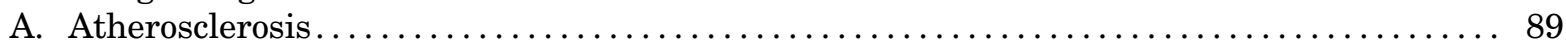

1. Clinical Evidence for WNT Signaling in Atherosclerosis. .................... 89

2. WNT Signaling and Endothelial Dysfunction......................... 89

3. WNT Signaling in Macrophages. .................................... 91

4. WNT Signaling in Vascular Smooth Muscle Cells. . . . . . . . . . . . . . . . . . . . 92

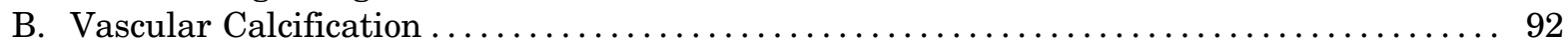

C. Norrie Disease ............................................... 93

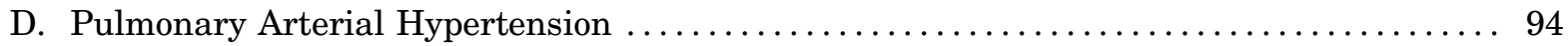

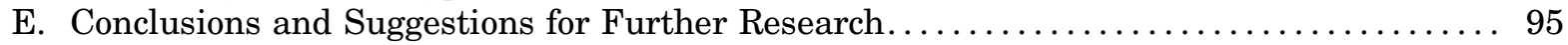

VIII. WNT Signaling in Cardiac Disease .................................... 95

A. WNT Signaling in Myocardial Infarction.............................. 95

1. Effects of Modulation of WNT Expression and Secretion.................... 95

2. Modulation at the Level of Secreted Frizzled-Related Proteins................. 96

3. Interventions at the Receptor Complex. ............................ 97

4. The $\beta$-Catenin Destruction Complex. .............................. 98

5. $\beta$-Catenin-Mediated Gene Transcription. ........................... 98

B. WNT Signaling in Valvular Disease $\ldots \ldots \ldots \ldots \ldots \ldots \ldots \ldots \ldots \ldots \ldots \ldots \ldots \ldots \ldots$

C. WNT Signaling in Cardiac Arrhythmias ................................. 100

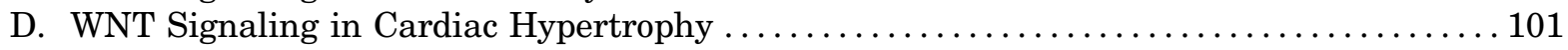

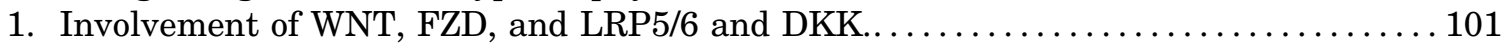

2. The Role of Secreted Frizzled-Related Proteins in Cardiac Hypertrophy. ........... 102

3. The $\beta$-Catenin Destruction Complex. .............................. 102

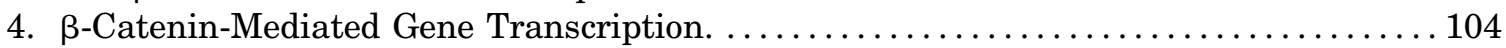

E. WNT Signaling and Heart Failure .................................. 105

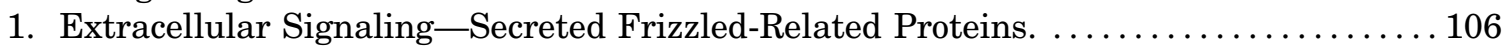

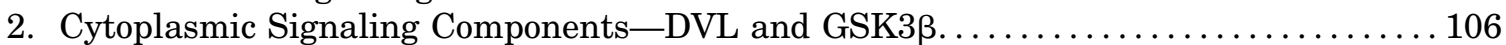

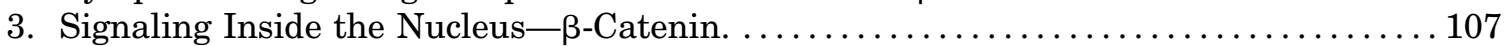

F. Conclusions and Directions for Future Research $\ldots \ldots \ldots \ldots \ldots \ldots \ldots \ldots \ldots \ldots \ldots \ldots \ldots \ldots$

IX. WNT Signaling in Stem Cells ......................................... 107

A. WNT Signaling Controls Stem Cell Differentiation......................... 108

B. WNT Signaling and Cardiovascular Regeneration in Lower Vertebrates .............. 108

C. WNT Signaling in Cardiomyocyte Differentiation of Stem/Progenitor Cells ............ 108

D. WNT Signaling in Cardiac Fibrosis and Repair ................................ 109

E. Conclusions and Future Perspectives ................................ 109

X. Crosstalk between WNT Signaling and Other Signal Transduction Pathways ............ 109

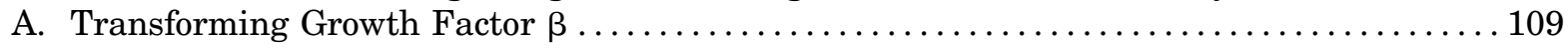

1. Extracellular Crosstalk between WNT and TGF $\beta$ Signaling. .................. 109

2. Crosstalk at the Level of the Signal Transduction........................... 109

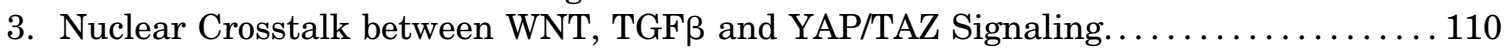

B. Renin Angiotensin System . .................................... 110

C. Vascular Endothelial Growth Factor................................... 111

\begin{abstract}
ABBREVIATIONS: AC, arrhythmogenic cardiomyopathy; AGE, advanced glycation end product; AngII, angiotensin II; APC, adenomatous polyposis coli; AR, androgen receptor; BBB, blood-brain barrier; BIO, 6-bromoindirubin-3'-oxime; BMP, bone morphogenetic protein; BNP, brain natriuretic peptide; BRB, blood-retina barrier; CBP, CREB binding protein; CK1, casein kinase 1; CNS, central nervous system; COX-2, cyclooxygenase-2; CRD, cysteine-rich domain; Cx, connexin; DEP, disheveled-EGL10-plekstrin; DIX, disheveled-axin; DKK, dickkopf; DVL, disheveled; E, embryonic day; EC, endothelial cell; EMT, epithelial-mesenchymal transformation; FZD, frizzled; GSK3, glycogen synthase kinase-3; HUVEC, human umbilical vein endothelial cells; IL, interleukin; IWR, inhibitors of WNT response; JNK, c-Jun NH kinase; KO, knockout; LDL, low-density lipoprotein; LGR, leucine-rich repeat-containing G protein-coupled receptor; LiCl, lithium chloride; LRP, lipoprotein-related receptor protein; MI, myocardial infarction; MMP, matrix metalloproteinase; MSC, mesenchymal stem cells; NFAT, nuclear factor of activated T-cells; ox, oxidized; PAH, pulmonary arterial hypertension; PASMC, pulmonary artery smooth muscle cells; PCP, planar cell polarity; PKC, protein kinase C; PLVAP, plasmalemma vesicle-associated protein expression; PORCN, porcupine; PRR, (pro)renin receptor; RAGE, receptor for advanced glycation end products; RAS, renin angiotensin system; ROR, receptor tyrosine kinase-like orphan receptor; sFRP, secreted frizzled-related protein; SHF, second heart failure; SHHF, spontaneously hypertensive rat heart failure; TCF/LEF, T-cell factor/lymphoid enhancer factor; TM, transmembrane; TNF, tumor necrosis factor; TNK, tankyrases; VEGF, vascular endothelial growth factor; WD, windorphen; WIF, WNT inhibitory factor; WLS, Wntless; WT, wild type.
\end{abstract}




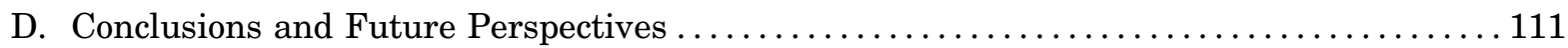

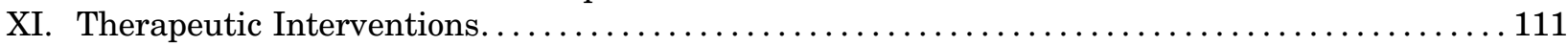

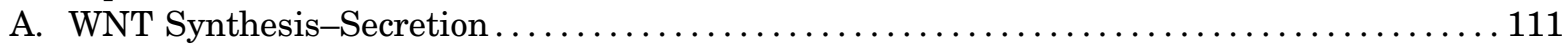

B. Extracellular Pharmacological Targeting of WNT Signaling: WNT-FZD Interaction ..... 112

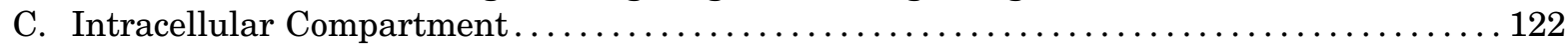

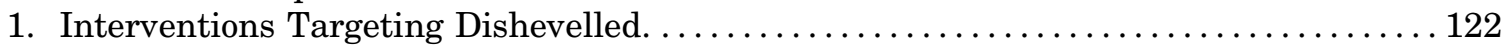

2. Interventions Targeting Axin. ........................................ 122

3. Interventions Targeting CK1 ....................................... 124

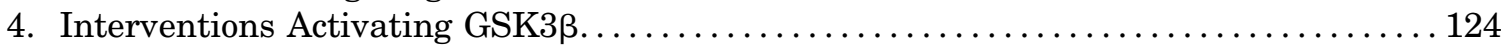

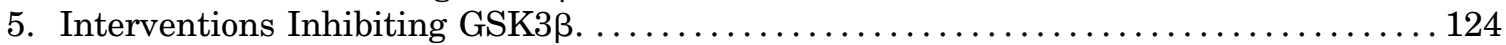

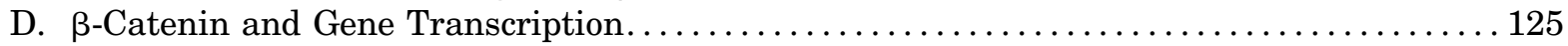

1. Targeting of the T-cell Factor/Lymphoid Enhancer Factor Transcription Factors. . . . 126

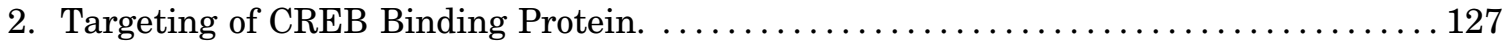

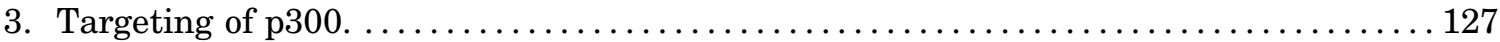

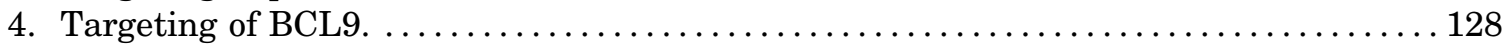

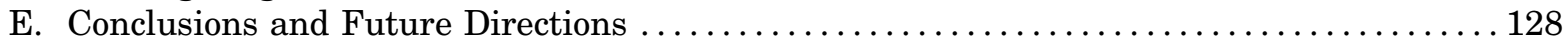

XII. Future Perspectives................................................. 129

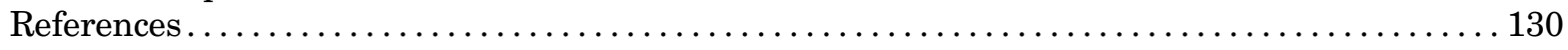

\begin{abstract}
WNT signaling is an elaborate and complex collection of signal transduction pathways mediated by multiple signaling molecules. WNT signaling is critically important for developmental processes, including cell proliferation, differentiation and tissue patterning. Little WNT signaling activity is present in the cardiovascular system of healthy adults, but reactivation of the pathway is observed in many pathologies of heart and blood vessels. The high prevalence of these pathologies and their significant contribution to human disease burden has raised interest in WNT signaling as a potential target for therapeutic intervention. In this review, we first will focus on the constituents of the pathway and their regulation and the different signaling routes. Subsequently, the role of WNT signaling in cardiovascular development is addressed, followed by a detailed discussion of its
\end{abstract}

involvement in vascular and cardiac disease. After highlighting the crosstalk between WNT, transforming growth factor- $\beta$ and angiotensin II signaling, and the emerging role of WNT signaling in the regulation of stem cells, we provide an overview of drugs targeting the pathway at different levels. From the combined studies we conclude that, despite the sometimes conflicting experimental data, a general picture is emerging that excessive stimulation of WNT signaling adversely affects cardiovascular pathology. The rapidly increasing collection of drugs interfering at different levels of WNT signaling will allow the evaluation of therapeutic interventions in the pathway in relevant animal models of cardiovascular diseases and eventually in patients in the near future, translating the outcomes of the many preclinical studies into a clinically relevant context.

\section{Introduction}

Cardiovascular diseases include a wide variety of conditions, such as atherosclerosis leading to tissue ischemia, cardiac hypertrophy, valvular defects, and cardiac arrhythmias. At a first glance, these conditions may seem to have only few similarities, but the underlying disease mechanisms such as inflammation, fibrosis, and endothelial dysfunction are common denominators in cardiovascular diseases. This is also illustrated by the fact that, despite the apparent differences in the symptoms, the current pharmacotherapeutic arsenal for the treatment of these conditions is remarkably similar. Inhibitors of the renin-angiotensin system, $\mathrm{Ca}^{2+}$-antagonists, inhibitors of the sympathetic nervous system, diuretics, and statins form the cornerstones of the therapy, supplemented with anticoagulants where needed. Although these drugs have been proven to be useful in treating the symptoms of many cardiovascular diseases, their effect on disease progression is limited. Moreover, recent efforts to develop new drugs for cardiovascular diseases, particularly for heart failure, have not been very successful (Vaduganathan et al., 2013).

Obviously, the ideal cardiovascular therapy would induce regression of the disease process and eventually cure the patient. This illustrates that there is a need for a better understanding of the underlying signaling mechanisms involved in the disease process. In this review we will discuss the role of the WNT signal transduction pathway in cardiovascular diseases. WNT signaling is well known for its role in developmental biology (van Amerongen and Nusse, 2009), but there are many indications that the pathway is reactivated in disease (Clevers and Nusse, 2012). In this review, we will first provide an overview of the components of the cascade and their regulation, followed by their interactions in the different signaling pathways and a description of their role in the development of the cardiovascular system. Subsequently, we will provide an overview of the experimental evidence for involvement of WNT signaling in vascular and cardiac pathology. 
Next, we will discuss the role of WNT signaling in stem cell maintenance and differentiation, a relatively new field that may hold promise for the regeneration of defective or malfunctioning tissue. Finally, we will provide an overview of the drugs developed for pharmacological intervention at different levels of the signaling cascade. In this review, we will use the term "WNT signaling" to refer to the pathway as a whole in all its complexity. Specific signaling routes will be indicated by " $\beta$-catenin-mediated WNT signaling" or "WNT/ $\beta$-catenin signaling" and "non- $\beta$-catenin-mediated WNT signaling," respectively.

\section{The History of WNT Signaling}

It is now more than 30 years ago that Nusse and Varmus (1982) published the first report on a gene they discovered in a search for preferential integration sites for mouse mammary tumor virus. Although the sequence of the gene, which they named int1, did not share any homology with other genes known at that time, overexpression studies established that int 1 behaved as a bona fide protooncogene (Tsukamoto et al., 1988). The int1 gene displayed a high degree of conservation across species, which appeared to be key in identifying the role of this gene (Nusse and Varmus, 2012). Around the same time, Nusslein-Volhard and Wieschaus (1980) investigated the molecular mechanisms leading to developmental mutants of Drosophila. They identified a class of segment-polarity genes, showing similar patterning defects when mutated. The names of these genes were derived from the description of their phenotype, such as Wingless, Armadillo, and Arrow. Cloning and sequencing of both the int1 and Wingless genes revealed that they were in fact homologs, making them one of the first examples of a gene that is active in both normal development and in malignancies (Rijsewijk et al., 1987).

Expression of WNT proteins has been observed during the development of multicellular organisms throughout the animal kingdom, but not in plants. Multiple orthologs of WNT genes are found in most animals, with a staggering total of 19 WNT genes in mouse and man subdivided into 12 conserved subfamilies (Kusserow et al., 2005). WNT proteins have the capacity to induce polarization of cells by activating the planar cell polarity (PCP) pathway and to activate their lineage-specifying signaling, two prerequisites for the development of an asymmetric shape and cellular diversity of metazoans (Loh et al., 2016). Over time, activation of more pathways, including the $\mathrm{WNT} / \beta$-catenin (frequently referred to as "canonical" WNT signaling) and WNT/Ca ${ }^{2+}$ signaling, have been identified.

Over the years, research on WNT signaling has been hampered by several experimental difficulties. First, there was the problem of the purification of WNT proteins. The transportation of WNT proteins to the extracellular compartment appeared to be a complicated process, involving the covalent linking of a palmitoleate moiety, which is essential for the biologic activity of the protein. It took until 2003 before a protocol was developed that allowed the purification of active WNT protein (Willert et al., 2003). The development of specific antibodies for WNT proteins has also been a timeconsuming and frustrating endeavor (Nusse and Varmus, 2012). Moreover, it took more than a decade to identify proteins from the frizzled (FZD) family to act as the principal receptors for WNT proteins (Wang et al., 2016a) The identification of coreceptors, multiple signaling pathways, and endogenous antagonists further have contributed to our knowledge on this intriguing yet complex regulatory network.

The role of WNT signaling in disease has been an area of intense research in the last decades. Some 20 years ago, mutations in the human adenomatous polyposis coli (APC) gene were found to lead to multiple polyps in the colon, significantly increasing the risk of developing colon carcinoma (Kinzler and Vogelstein, 1996). This observation led to the discovery of $\beta$-catenin as an intracellular signaling protein in the $\mathrm{WNT} / \beta$-catenin signaling pathway (Korinek et al., 1997). In the meantime, WNT signaling has been linked to many diseases, including osteoporosis, neurodegenerative diseases, and cardiovascular diseases (Hermans and Blankesteijn, 2015). It is important to note, however, that in most of these diseases an aberrant activation of the pathway rather than a mutation of one of its components is contributing to the disease process. This may have important implications for the choice of the therapeutic target, as will be discussed in section XI of this review.

\section{Components of the Receptor Complex}

A. Synthesis and Posttranslational Modifications of WNT

WNTs are secreted proteins for which homologs have been detected in multicellular organisms ranging from sponges to vertebrates. They contain $22-24$ conserved cysteine residues, responsible for the maintenance of the spatial structure by the formation of disulfide bonds. The 19 WNT genes identified in mammals range in molecular weight from $39-46 \mathrm{kDa}$ and belong to $12 \mathrm{sub}-$ families: many WNT genes (including WNT2, $-3,-5,-7$, $-8,-9$, and -10 ) have shown duplications, giving rise to two closely related family members (e.g., WNT $3 \mathrm{a}$ and WNT 3b). Wnts can also be classified according to their function. A large group of Wnts is capable of inducing oncogenic transformation and duplication of the body axis in developing Xenopus embryos (WNT1, -3a, -7a, $-7 b,-8)$, whereas another group does not (e.g., WNT4, $-5 \mathrm{a},-6$, and -11). This distinction is related to different signal transduction pathways that are activated by these WNTs, as will be further discussed in section $V$ of this review (Croce and McClay, 2008). However, it has 
to be noted that not only the subtype of WNT protein, but also the FZD family member and probably also the coreceptors, are important in determining the choice of the signaling pathway (Schulte, 2015).

WNT proteins are subjected to extensive posttranslational modification to become secreted in a biologically active form. All WNT proteins carry a signal sequence that is required for their secretion (Langton et al., 2016). All WNT proteins except Drosophila WntD carry a palmitate group at a conserved cysteine residue located near the $\mathrm{N}$ terminus $\left(\mathrm{Cys}^{77}\right.$ in WNT3A) and a palmitoleate group at a conserved serine residue $\left(\mathrm{Ser}^{209}\right.$ in WNT3A). Originally, the palmitate was thought to be necessary for receptor binding, internalization, and signaling, whereas the palmitoleate group was thought to be required for intracellular transport (Willert et al., 2003; Schulte, 2010; Harterink and Korswagen, 2012). However, after the resolution of the crystal structure of the $\mathrm{xWnt} 8 / \mathrm{Fzd}_{8}$ complex, WNT was shown to have a three-dimensional structure resembling a hand, with two extension resembling the thumb and index finger grasping the CRD of FZD. The palmitoleated $\mathrm{Ser}^{209}$ residue was found to be located at the tip of the so-called "thumb" region, which, together with the index finger, has been shown to be involved in the interaction of WNT proteins with receptors from the FZD family (Janda et al., 2012; Langton et al., 2016). Since the palmitated $\mathrm{Cys}^{77}$ residue is not located at a distinctive region involved in receptor interaction, the role of its lipidation has become less clear. However, the addition of a hydrophobic group may help to concentrate active
WNT proteins at the cell membrane, which may assist in activation of signaling (Kaemmerer and Gassler, 2016). The addition of the palmitoleate group to WNT proteins takes place in the endoplasmic reticulum by an enzyme called porcupine (PORCN). PORCN is a member of the membrane-associated $O$-acyl transferase enzymes, which promote the coupling between a palmitoleate and the conserved serine residue in the thumb region. Next to lipidations, WNT proteins can also be glycosylated at two specific sites (Lorenowicz and Korswagen, 2009). For WNT3A, this glycosylation was found to precede the palmitoyleation (Komekado et al., 2007). The glycosylation appears to be critical in controlling WNT folding and secretion, but is not essential for WNT signaling (Willert and Nusse, 2012).

Apart from its importance in binding to FZD, attaching a palmitoleate to the WNT protein also plays a crucial role in the secretion of WNT proteins because it stimulates the binding of WNTs to Wntless (WLS), a chaperone protein that escorts the palmitoleated WNT to the plasma membrane (Lorenowicz and Korswagen, 2009; Langton et al., 2016). As illustrated in Fig. 1, the binding of WNT to WLS occurs in the endoplasmic reticulum and is essential for the progression through the Golgi network. When reaching the plasma membrane, the WNT/WLS complex dissociates and WLS is recycled back to the Golgi apparatus by the retromer complex, which is responsible for endosome to Golgi trafficking (MacDonald et al., 2009; Harterink and Korswagen, 2012). Interestingly, WNT proteins have been shown to be released in a polarized fashion in

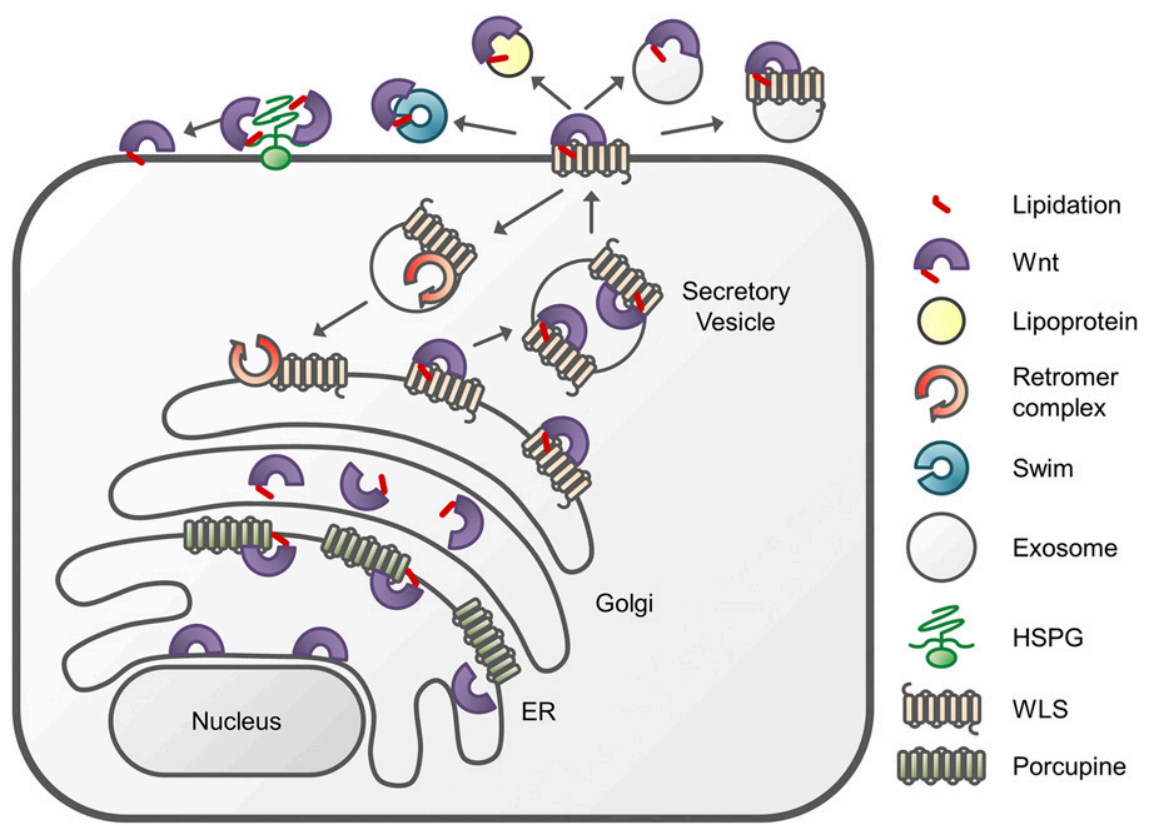

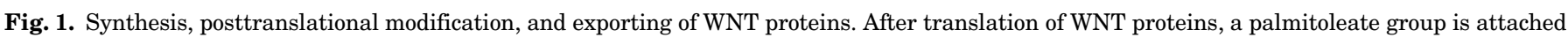

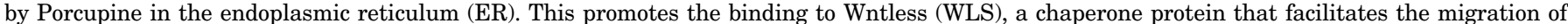

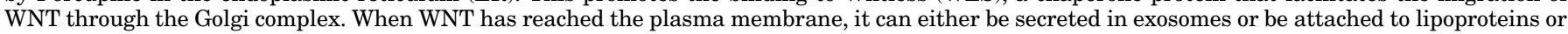

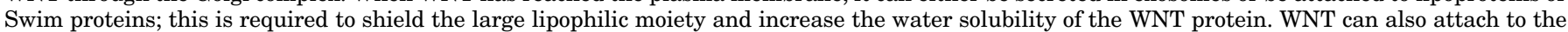

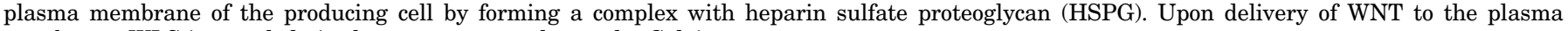
membrane, WLS is recycled via the retromer complex to the Golgi apparatus. 
epithelial cells, with different secretion profiles on the basal and apical sides of the cell (Lorenowicz and Korswagen, 2009; Langton et al., 2016). However, there is still debate whether secretion of WNT proteins from cells is actually necessary for their biologic activity, because WNTs have been found attached to cytonemes, actin-based cellular extensions that extend distances of several hundreds of micrometers (Stanganello and Scholpp, 2016).

Due to the attachment of the lipophilic groups, the solubility of WNT proteins in an aqueous environment is poor. Therefore, four mechanisms have been proposed for the secretion of WNT proteins into the extracellular compartment: 1) association with a lipoprotein particle such as lipophorin in Drosophila (Panakova et al., 2005); 2) binding to the secreted WNT-interacting molecule Swim, a member of the lipocalin family (Mulligan et al., 2012); 3) spreading of WNT over the plasma membrane with the lipid group inserted in the membrane (Stanganello et al., 2015); and 4) secretion of WNT proteins in exosomes (Gross et al., 2012). The first two mechanisms are thought to increase the water solubility by shielding the palmitoleate group, whereas in the latter two mechanisms this group is inserted in the hydrophobic membrane environment (Langton et al., 2016).

\section{B. Frizzled Proteins}

The name "frizzled" is derived from a Drosophila homolog of this gene, the mutants of which give rise to a distinctive derangement of the orientation of cutical wing hairs and bristles (Gubb and Garcia-Bellido, 1982). Up until now, 10 FZD homologs have been identified in mammals. On the basis of structural homology, they can be clustered in five subfamilies: $\mathrm{FZD}_{1 / 2 / 7}, \mathrm{FZD}_{5 / 8}, \mathrm{FZD}_{9 / 10}, \mathrm{FZD}_{4}$, and $\mathrm{FZD}_{3 / 6}$; smoothened is a protein structurally related to the FZDs but no member of the FZD family. Major differences exist in the genetic structure of the different $F Z D$ genes with $F Z D_{3}$ and $F Z D_{6}$ having five introns in their coding region and $F Z D_{4}$ having a single exon, whereas the other FZDs are intronless.

FZD proteins are a family of seven transmembrane (TM) receptors, meaning that there are seven helices of hydrophobic amino acids present in the protein allowing its embedding in a membranous structure. Although this feature is similar to many other members of the class of $\mathrm{G}$ protein-coupled receptors, the International Union of Basic and Clinical Pharmacology has categorized FZD proteins into a separate class, together with the smoothened protein, a member of the Hedgehog family (Foord et al., 2005). The reason for this classification is that FZDs have a unique extracellular domain at the N-terminal side, in which five disulfide bonds formed by 10 highly conserved Cys residues determine the three-dimensional shape. This so-called cysteine-rich domain (CRD) is thought to be the site of interaction with WNT proteins. Interestingly, similar CRDs are present in other non-seven transmembrane (7TM) proteins such as soluble frizzled-related proteins (sFRPs), the receptor tyrosine kinase-like orphan receptor (ROR), carboxypeptidase $\mathrm{Z}$ and a splice variant of collagen XVIII. For several of these proteins, interaction with WNT has actually been demonstrated (Xu and Nusse, 1998). Upstream of the CRD, the N-terminal part of the FZD proteins contains a signal sequence, necessary for transport to the plasma membrane, which is cleaved off in the active receptor protein. The CRD of the receptor is connected to the 7TM domain by means of a spacer of variable length (Schulte, 2010). On the intracellular side of the receptor, three loops and the free C-terminal end of the protein are present as in all $G$ protein-coupled receptors. Although several domains important for $\mathrm{G}$ protein coupling still need to be defined (Gammons et al., 2016), there is increasing evidence that, among coupling to other signal transduction pathways, G protein signaling can actually be activated by FZD proteins, as discussed in more detail in section $V . E$ of this review (Dijksterhuis et al., 2014). A remarkable feature of the C-terminal part of the FZDs is the fully conserved KTxxxW domain, located at the eighth helix (Gammons et al., 2016). Mutation experiments have shown that this domain is indispensable for interaction with the PDZ-domain of disheveled (DVL), a protein involved in most WNT/FZD signal transduction pathways (Schulte, 2010).

Targeted mutagenesis experiments have revealed that FZDs can show significant functional redundancy within their subfamily, so mice lacking a single FZD gene quite frequently show no clear phenotype. In the context of this review, we focus on the (combinations of) deletions that give rise to a cardiovascular phenotype; for a comprehensive overview of the phenotypes of all FZD knockouts, we refer the reader to the recent review by Wang et al. (2016a). When focusing on the $\mathrm{FZD}_{1 / 2 / 7}$ subfamily, $F z d_{1}^{-/-}$mice show no clear phenotype, whereas about half of the $F z d_{2}^{-1-}$ mice show a cleft palate and $\pm 15 \%$ of the $F z d_{7}^{-1-}$ have a ventricular septal defect. Combining the different targeted mutants aggravates the frequency of the phenotypes: all $F z d_{1}^{-1-}$; $F z d_{2}^{-1-}$ double knockouts were found to have a cleft palate and the majority of them had cardiac defects as well, ranging from a ventricular septal defect to a double outlet right ventricle (Yu et al., 2010). The combination of $F z d_{2}^{-I-} ; F z d_{7}^{-1-}$ was found to be embryonically lethal due to failure of closing its neural tube and failure to develop cardiac asymmetry, impairing cardiac function (Yu et al., 2012). These results demonstrate that adequate signaling via the $\mathrm{FZD}_{1 / 2 / 7}$ subfamily is a prerequisite for a correct development of the heart.

Loss of $\mathrm{FZD}_{4}$ has been shown to reduce vascular cell proliferation and migration. Moreover, tube formation by endothelial cells (ECs) is impaired, resulting in a diminished arterial network in heart and kidney 
(Descamps et al., 2012). A severe defect in retinal vascularization was observed in mice lacking $\mathrm{Fzd}_{4}$ (Wang et al., 2012). This is associated with the progressive development of hearing loss and degeneration of the cerebellum, the latter caused by leakage of the blood-brain barrier in these mice (Wang et al., 2001). The combined findings stress the importance of proper $\mathrm{FZD}_{4}$ signaling for the development and maintenance of vascular function, which will be further elaborated in section VII.C.

\section{Lipoprotein-Related Receptor Protein 5/6}

After the identification of FZD proteins as receptors for WNT, further research revealed that additional binding partners are required to activate $\mathrm{WNT} / \beta$-catenin signaling. This led to the simultaneous publication by three independent research groups of two members of the lowdensity lipoprotein receptor family. These proteins are called low-density lipoprotein-related receptor (LRP) 5 and 6 and are homologs of Drosophila Arrow (Pinson et al., 2000; Tamai et al., 2000; Wehrli et al., 2000). These receptor proteins are over 1600 amino acids in size and have a single TM domain. The bulky extracellular part of LRP5 and 6 has a modular structure consisting of four six-bladed $\beta$-propellers connected by EGF-like domains. A flexible hinge region between the two pairs of $\beta$-propellers allows for some bending of the protein, giving rise to a concave top surface of the extracellular domain (Chen et al., 2011; Cheng et al., 2011b). The $\beta$-propeller part is connected to the transmembrane region via three LDL type A repeats. Overexpression of the LRP6 intracellular domain can activate WNT signaling in a constitutive fashion, confirming the regulatory role of the extracellular domain in the control of the signal transduction (Niehrs and Shen, 2010).

The intracellular part of LRP5/6 contains multiple phosphorylation sites, the phosphorylation of which is a key step in the initiation of the signal transduction via $\mathrm{WNT} / \beta$-catenin signaling. Several kinases have been found to phosphorylate LRP6, either in a WNTinducible ( $\mathrm{G}$ protein receptor kinase $5 / 6$ ) or WNTindependent (protein kinase A, PFTAIRE protein kinase 1) way. For an overview, we refer to a review article on this topic (Niehrs and Shen, 2010).

WNT proteins can bind to LRP5/6 at two different sites, albeit with lower affinity than to FZD proteins (Tamai et al., 2000). The most $\mathrm{N}$-terminal $\beta$-propeller can bind most WNT members, with the exception of WNT3 and WNT3A that bind to the third $\beta$-propeller. Several endogenous inhibitors of LRP5 and 6, including members of the dickkopf (DKK) family, SOST and WISE, were shown to interact with the first $\beta$-propeller, whereas DKK proteins also bind to the third $\beta$-propeller. Interestingly, antibodies specific for each of the two binding sites have been generated, allowing the specific blocking of each site (Joiner et al., 2013).
Mice lacking either the Lrp5 or - 6 gene show distinct phenotypes. Inactivating mutations of Lrp5 led to low bone mass and abnormal retinal vascularization, combined with defects in cholesterol and glucose metabolism. Delays in mammary development and resistance to WNT1-induced mammary tumorigenesis have also been reported. Global deletion of Lrp6 led to postnatal lethality with skeletal, neurologic, and mammary gland defects and cardiac abnormalities including a double outlet right ventricle. Double mutants show abnormal posterior patterning of the epiblast. Several mutations in LRP5 and - 6 are linked to human diseases, including osteoporosis pseudoglioma, Alzheimer's disease and degenerative joint disease (Joiner et al., 2013). Similar to the phenotype of the $F Z D_{4}$ mutant described above, mutations in LRP5 can lead to familial exudative vitreoretinopathy (Toomes et al., 2004). The R611C mutation in LRP6 was found to be associated with elevated serum lipids and glucose, hypertension, and premature coronary artery disease (Mani et al., 2007). Moreover, increased LRP5 levels have been associated with calcified aortic valves, a finding closely linked to the importance of WNT signaling in bone formation, as described in more detail in section VIII.B (Rajamannan, 2011).

\section{Receptor Tyrosine Kinase-like Orphan Receptor $1 / 2$ and Ryk}

ROR1 and - 2 were initially identified because of their layout showing similarity to tyrosine kinase receptors. These receptor proteins contain a single TM domain and the intracellular parts display kinase activity. Unlike other tyrosine kinase receptors, however, these receptor proteins were found to have an extracellular CRD domain that closely resembles the CRDs of FZD proteins (Saldanha et al., 1998). This suggests that WNT proteins serve as ligands for ROR1 and -2 , which was indeed shown to be the case (Oishi et al., 2003). In the meantime, it has become clear that, in contrast to LRP5/6, ROR1 and -2 are mostly involved in $\beta$-catenin-independent WNT signaling (Green et al., 2014).

Ror2 null mice show skeletal defects, abnormal orientation of inner ear hairs, and abnormalities in lungs and ventricular septal defects (Takeuchi et al., 2000). The phenotype of Ror1-deficient mice is less severe, but deletion of Ror 1 augments the phenotype of Ror2 deficiency and introduces additional defects such as transposition of the great arteries. This suggests a genetic interaction between Ror 1 and -2 in cardiac and skeletal development (Nomi et al., 2001).

Ryk proteins also have a single membrane-spanning domain, like the ROR proteins, but contain an extracellular domain with homology to WNT inhibitory factor (WIF) rather than the CRD found in FZD and ROR proteins. Moreover, Ryk also deviates from RORs in that its intracellular domain has no enzymatic activity. This suggests that Ryk most likely functions 
as a coreceptor for WNTs together with FZD proteins (Green et al., 2014).

\section{E. Interaction between WNT, FZD, and Their Coreceptors}

1. Physical Interaction between WNT and FZD. The selectivity and specificity of the WNT-FZD interaction is far from being fully understood. This obviously has to do with the large number of WNT and FZD homologs and the role of coreceptors in the binding and signal transduction. Moreover, the highly lipophilic nature of the WNT proteins forms an experimental challenge for traditional receptor-binding studies as it results in high levels of nonspecific binding (Dijksterhuis et al., 2014). Despite these technical difficulties, several authors reported experimental approaches to study the physical interaction between WNTs and the CRDs of different FZD homologs, including an enzyme-linked immunosorbent assay-based protein-protein interaction assay (Carmon and Loose, 2008, 2010) and immunoprecipitation (Sato et al., 2010a). Moreover, in many publications, functional assays such as TOPFlash/TOPGal reporter assays or receptor internalization were used as a readout for WNT/FZD interaction (Schulte, 2010). In most of these experiments, the affinity of the WNT/FZD interaction was found to be in the nanomolar range. From the available experimental evidence, of which a nice overview is provided by Dijksterhuis et al. (2014), it can be deduced that most FZDs can interact with multiple WNTs, whereas FZD ${ }_{9}$ and ${ }_{-10}$ appear to interact exclusively with WNT2 and WNT7B, respectively. Interestingly, there appears to be no such thing as "WNT/ $\beta$-catenin" or "WNT/ $\beta$-catenin independent" FZDs as both WNT3A (a prototype activator of WNT/ $\beta$-catenin signaling) and WNT5A (a prototype activator of WNT/ $\beta$-catenin independent signaling) can bind to the vast majority of FZDs. However, a word of caution is in place here: the fact that a physical interaction between certain WNTs and FZDs can be detected under artificial experimental conditions does not necessarily mean that these interactions also occur in vivo and/or can lead to signaling under physiologic conditions.

2. Structural Data. Despite the publication of the structure of the CRD of the FZD protein (Dann et al., 2001), little was known about the site(s) of interaction between WNT and FZD until the publication of the crystal structure of a glycosylated Xenopus Wnt8/mouse $\mathrm{Fzd}_{8}$-CRD complex (Janda et al., 2012). A first hint toward the mode of interaction could be derived from the observation that the water solubility of this complex was far better than for xWnt8 alone, suggesting a shielding of the hydrophobic palmitoleate group by the $\mathrm{mFzd}_{8}$ CRD. This allowed the crystallization of the $\mathrm{xWnt} 8 / \mathrm{mFzd}_{8}{ }$-CRD complex in a detergent-free environment. In this complex, Wnt appears to be shaped like a hand where the two ends, conveniently named "thumb" and "index finger," grasp the $\operatorname{mFzd}_{8}$ CRD. The thumb region, consisting of amino acids 185-195, contains two disulfide bridges stabilizing the tertiary structure of the loop. The palmitoleate group, located at the tip of the thumb region, directly fits into a groove located in the $\mathrm{mFzd}_{8}-\mathrm{CRD}$ in which multiple nonpolar amino acids are present for interaction with the lipid extension. These amino acids appear to be present in all FZD CRDs, suggesting a conserved mode of interaction between multiple WNTs and FZDs.

The site of interaction between the index finger of $\mathrm{xWnt8}$ and the CRD of $\mathrm{mFzd}_{8}$ is located on the opposite side of the horseshoe and consists of a loop formed by the amino acid residues $\mathrm{Arg}^{301}$ to $\mathrm{Cys}^{338}$. The structure of the index finger consists of a long beta strand in which three disulfide bonds maintain the tertiary structure. The tip is characterized by an unusual disulfide tandem formed by $\mathrm{Cys}^{320}, \mathrm{Cys}^{321}$, and a $\operatorname{Trp}^{319}$ residue, the side chain of which fits in pocket on the $\mathrm{FZD}_{8}$ surface. The C-terminal part of the $\mathrm{xWnt8}$ protein, containing only the index finger, could bind to several FZD CRDs with variable affinity, suggesting that this interaction may be responsible for selectivity of Wnt/FZD interaction (Janda et al., 2012).

3. Interaction with Coreceptors. The sites of interaction between WNTs and their coreceptors, such as LRP5/6, Ryk, and ROR2, are not yet resolved by crystallography but based on the identification of conserved regions. An analysis of the 19 WNT sequences showed a large patch of high conservation, located at the top of the central part of the WNT protein. This patch contains three short but highly conserved and solvent-exposed stretches of amino acids that localize in close proximity in the top central part of the WNT protein and was predicted to be the interaction site for the coreceptors. However, experimental evidence that this site is actually binding to the coreceptors still has to be provided (Janda et al., 2012).

4. Formation of Multimeric Complexes. An increasing number of $\mathrm{G}$ protein-coupled receptors has been shown to be able to aggregate into homologous or heterologous oligomeric complexes, affecting their ligand binding, signaling, and regulation (Milligan, 2009). Several studies have shown the formation of multimeric FZD complexes (Schulte, 2015), which is sufficient to activate signaling (Carron et al., 2003). Spontaneous dimerization was also reported for $\mathrm{FZD}_{3}$ receptors in Xenopus, whereas the forced dimerization of the CRDS of $\mathrm{xFZD}_{7}$ induced activation of signal transduction in this species (Carron et al., 2003). Although the presence of CRD dimers appears to be contradicted by the 1:1 stoichiometry observed in the crystallography data for the xWnt8/FZD 8 complex described above, a computational modeling study showed the docking of a dimeric $\mathrm{FZD}_{1}$ CRD complex inside the U-shaped space formed by the thumb and index finger of WNT1 (Ain et al., 2013). Additional research will be 
required to identify the stoichiometry of WNT/FZD interaction under physiological conditions and the potential effects on signaling of single versus multimeric CRDs.

\section{Regulation of WNT Signaling}

WNT signaling is not only characterized by a large number of ligands, receptors, and coreceptors, but can also be regulated at many different levels. Regulation can take place by intervention in the synthesis and excretion of WNT (e.g., by Notum), its lipidation (e.g., by PORCN), by inactivation of the WNT protein (e.g., by Tiki), by scavenging of WNT proteins (e.g., by sFRPs), and by controlling the density of FZD proteins at the plasma membrane (e.g., by LGR5). Moreover, inhibitors of the LRP5/6 coreceptors (DKK, sclerostin) have been described to control signaling. Interestingly, non-WNTrelated activators of the signaling pathways, such as $\mathrm{R}$-spondins and norrin, have also been identified. In this review, we will focus on those regulatory mechanisms that have been described in mammals and are likely to have a link with human disease (Fig. 2).

\section{A. WNT/Notum/Tiki}

In addition to a complex intracellular machinery for the secretion of WNT proteins, there are several extracellular mechanisms controlling the activity of secreted WNT proteins. Membrane-associated glypicans (named Dally in Drosophila) can bind WNT proteins to the cell surface, regulating their extracellular distribution and signaling activity. Adenomatous polyposis coli downregulated 1 (APCDD1) has a similar mode of action and has been shown to inhibit WNT signaling in cell culture assays (Cruciat and Niehrs, 2013). More recently, WNTspecific metalloproteinases from the Tiki family have been described that can cleave the first 9-20 amino acids from amino-terminal side of WNT2B, $-3,-3 \mathrm{~A},-5 \mathrm{~A},-5 \mathrm{~B},-6$, $-8 \mathrm{~A},-8 \mathrm{~B}$, and -16 , thereby rendering them inactive (Zhang et al., 2016b). Finally, a carboxylesterase named Notum has been shown to enzymatically remove the palmitoleate from WNT proteins, which results in a loss of biologic activity (Kakugawa et al., 2015). The many mechanisms involved in the secretion and (in)activation of WNT proteins highlight the complexity and tight control of WNT activity.

\section{B. Secreted Frizzled-Related Proteins}

As the name implies, $\mathrm{sFRPs}$ resemble FZD proteins in that they contain a CRD at the N-terminal side of the protein that shows $30 \%-50 \%$ sequence similarity with the FZD CRD. The first sFRP, originally named SFRB, which later turned out to be sFRP3, was in fact discovered based on its homology to FZD proteins (Hoang et al., 1996). In humans, five different sFRPs have been identified varying in size from 295 to 346 amino acids (Cruciat and Niehrs, 2013). The CRD contains 10 conserved Cys residues; the disulfide bridges formed by these residues contribute to the tertiary structure of the CRD. At the C-terminal side of the protein, a Netrin-related motif is located containing six conserved Cys-residues that form three disulfide

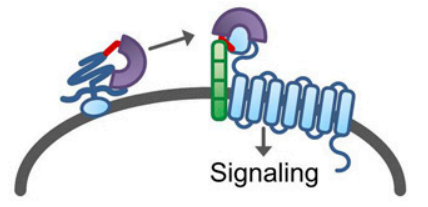

A: No inhibition

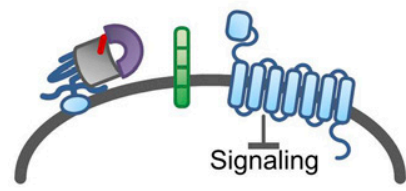

D: WIF

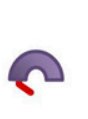

Wnt
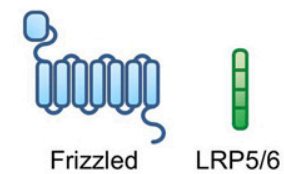

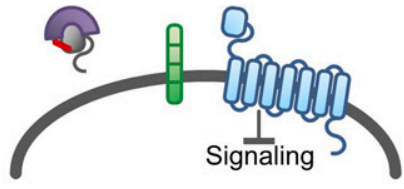

B: sFRP

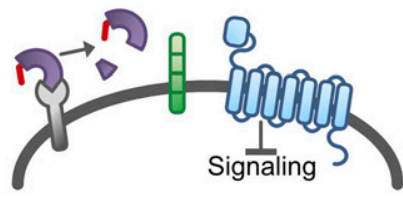

E: Tiki

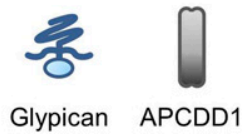

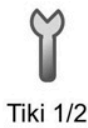

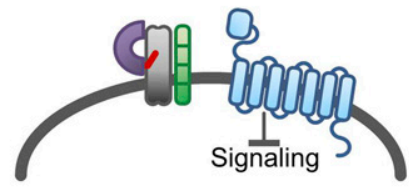

C: APCDD1

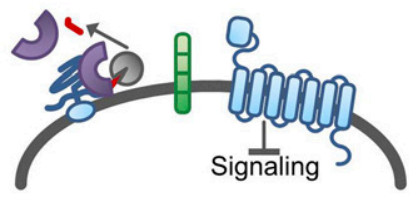

F: Notum

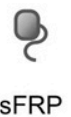

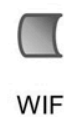

WIF
Notum

Fig. 2. Mechanisms of regulating WNT signaling activity. (A) In the absence of regulating mechanisms, WNT can bind to the FZD/LRP5/6 complex and activate signaling. (B) In the classic view, soluble frizzled-related proteins (sFRPs) can bind WNT and prevent its interaction with the receptor complex. However, sFRPs can modulate Wnt signaling in different ways, as described in the text (C). Adenomatous polyposis coli downregulated-1 (APCDD1) is a membrane-bound glycoprotein that can bind WNT and prevent its interaction with the receptor complex. (D) WNT-inhibitory factor (WIF) is a secreted protein shown to attenuate WNT signaling by binding the protein that prevents the association with the receptor complex. (E) Tiki is a transmembrane protein with proteolytic activity, cleaving an peptide of 8-20 amino acids from the N-terminal part of WNT proteins. This causes the formation of oligomers of WNT proteins that are inactive in signal transduction. (F) Notum is a carboxylesterase capable of removing the palmitoleic acid residue from WNT, making the protein biologically inactive. 
bridges (Chong et al., 2002). Netrin-related motifs are present in many unrelated proteins, including the axonguidance molecule Netrin, tissue inhibitors of metalloproteinases (TIMPs), type-1 procollagen C-protein enhancer proteins, and complement proteins $\mathrm{C} 3, \mathrm{C} 4$, and C5 (Cruciat and Niehrs, 2013). The sFRPs can be classified into two subgroups based on phylogenetic analysis: the sFRP1, -2 , and -5 subgroup and the sFRP3 and -4 subgroup (Jones and Jomary, 2002). Posttranslational modifications have been observed in sFRP1 and -5 ( $N$-glycosylation and sulphation of two Tyr residues), but these are absent in sFRP2, -3 , and -4 (Bovolenta et al., 2008).

The main mechanism of action of sFRPs is the inhibition of WNT signaling by scavenging extracellular WNT proteins, thereby preventing their interaction with the receptor complex. At first sight, the CRD region of the sFRPs appears to be responsible for this scavenging effect, as it can be anticipated to bind WNT proteins in a fashion similar to FZD proteins (Cruciat and Niehrs, 2013). However, structure-function analyses of sFRP1 has shown that the Netrin-related motif in the C-terminal region contributes to the inhibition of WNT function (Bhat et al., 2007; Bovolenta et al., 2008). The binding affinity for the interaction of sFRPs and WNT proteins has been reported to be in the nanomolar range, similar to the affinities of the WNT-FZD interaction. However, some specificity has been reported for the sFRP-WNT interaction: WNT3A binds to all sFRPs except sFRP5, whereas WNT5 only binds to SFRP1 and -2. As for the WNT-FZD interaction, so far no comprehensive studies have been published where all possible combinations were tested in a systematic way (Bovolenta et al., 2008).

In the meantime, several studies on sFRPs have offered conflicting results. Different sFRPs were shown to have opposing effects on apoptosis in breast tumors, and sFRP2 was shown to block the inhibitory effects of sFRP1 on kidney tubule formation (Bovolenta et al., 2008). Moreover, structural analysis of the CRDs of sFRPs has suggested that these CRDs might be able to form homo- or heterodimers with CRDs or either FZD or other sFRPs (Dann et al., 2001). Based on these observations, four mechanisms have been proposed by which sFRPs can modulate WNT signaling, two of which are inhibitory and two of which are stimulatory: 1) by scavenging WNT protein, which inhibits signaling because WNT is unavailable for interaction with its receptors; 2) by forming homo- or heteromeric sFRP complexes, which would favor WNT signaling; 3) by direct interaction with the CRDs of FZD, which would inhibit WNT signaling; and 4) by binding WNT to the Netrin-related motif, presenting WNT to the CRD of FZD, which would stimulate WNT signaling (Bovolenta et al., 2008). It is at present not clear how this control mechanism is directed in either of these four directions.

\section{WNT-inhibitory Factor}

WIF1 is a 379 -amino acid protein that has been shown to inhibit WNT signaling by binding WNT proteins (Hsieh et al., 1999). The protein contains a WIF domain, five EGF repeats, and a hydrophilic tail. In fact, the WIF domain is similar to that present at the extracellular end of the RYK tyrosine kinase receptor. WIF1 has been shown to interact with multiple WNTs from both the WNT1 (WNT/ $\beta$-catenin) and WNT5A (WNT/ $\beta$-catenin independent) subclasses. However, it is not clear what the mechanism of action is for the regulation of WNT signaling (Cruciat and Niehrs, 2013).

\section{Leucine-rich Repeat-containing G Protein-Coupled Receptor 4/5/R-Spondin}

Regulation of WNT signaling does not only depend on the amount of WNT available for receptor activation, but also on the receptor density on the cell membrane. A very effective mechanism for the regulation of the density of FZD proteins has been identified by analysis of WNT target gene expression (Fig. 3). Two highly homologous single transmembrane proteins, RNF43 and ZNRF3, were found to be upregulated as result of activation of WNT signaling. Both RNF43 and ZNRF3 are E3 ligases, capable of transferring ubiquitin groups to multiple lysine residues located in the intracellular loops of FZD proteins via the RING domain that is present in their cytoplasmic tail. This ubiquitination induces the endocytosis of the FZD proteins, making them inaccessible for ligand stimulation and causing an effective downregulation of WNT signaling (Hao et al., 2012; Koo et al., 2012).

Interestingly, the localization at the plasma membrane of the E3 ligases RNF43 and ZNRF3 is under the control of another group of WNT target genes, LGR4, -5, and -6. LGR5 was originally identified as a marker for intestinal stem cells (Barker et al., 2007). Together with LGR4 and -6 , these receptors form a subgroup of the leucine-rich repeat-containing $\mathrm{G}$ protein-coupled receptor (LGR) family. This seven-transmembrane receptor family is characterized by a large extracellular domain consisting of leucine-rich repeats, which is connected to the seven-transmembrane domain via a hinge region. Other members of this family are the receptors for follicle-stimulating hormone (LGR1), luteinizing hormone (LGR2), and thyroid-stimulating hormone (LGR3) (de Lau et al., 2014). The ligands for LGR4 and 5, members of the R-spondin family, are known as secreted enhancers of WNT signaling (de Lau et al., 2011). Binding of an R-Spondin to the leucine-rich repeat of LGR4/5 initiates the formation of a complex with RNF43 or ZNRF3, which is rapidly internalized. The loss of RNF43 and ZNRF3 from the plasma membrane leads to an attenuation of the ubiquitination of FZD, so more FZD remains at the plasma membrane to interact 

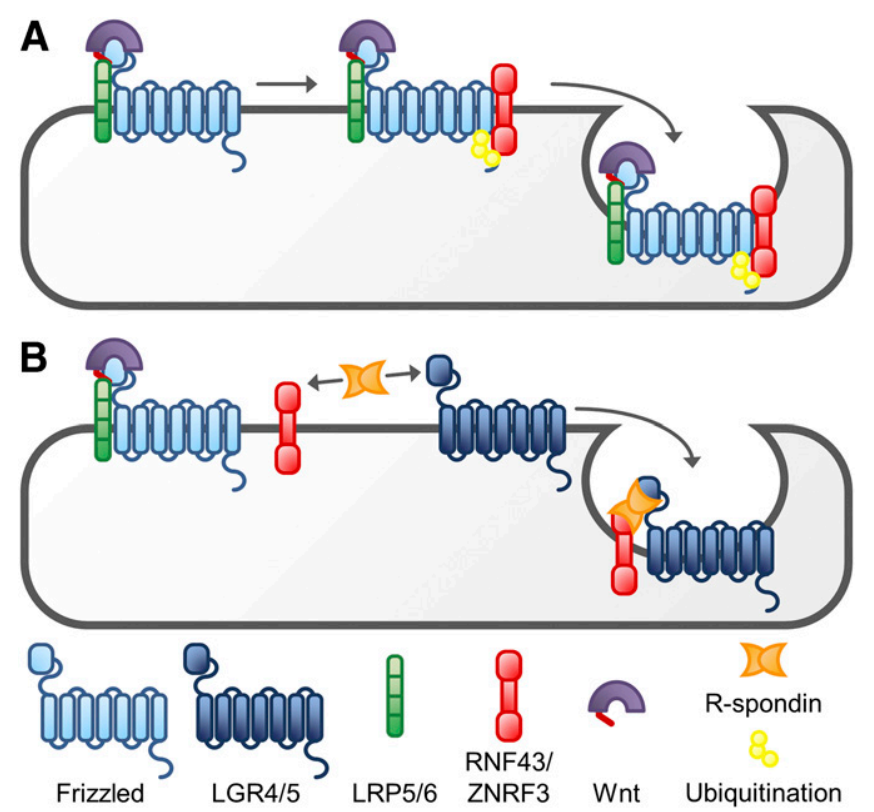

Fig. 3. Regulation of the amount of FZD protein on the plasma membrane. (A) Association of the transmembrane E3 ligases RNF43 and/or ZNRF3 with the FZD receptor complex induces its ubiquitination and leads to internalization of the receptor complex, making it unavailable for stimulation with WNT. (B) R-spondin is capable of redirecting the E3 ligases toward LGR4 or -5 , inducing the internalization of this transmembrane protein rather than the FZD/LRP5/6 complex. This effectively leaves more FZD receptors at the plasma membrane, available for stimulation with WNT proteins.

with WNT and activate the signal transduction (de Lau et al., 2014). It has to be noted, however, that an alternative mechanism has been described for the internalization of FZD4, i.e., via a DVL2-mediated recruitment of $\beta$-arrestin-2 (Chen et al., 2003).

\section{E. Dickkopf}

The first DKK protein was originally identified as an inducer of the Spemann organizer, a tissue with strong head-inducing capacity, which explains the name of the protein (dickkopf is German for big head) (Glinka et al., 1998). The DKK family consists of four members in vertebrates, named DKK1-4, varying in size between 255 and 350 amino acids. DKK1, -2 , and -4 have a high structural similarity with an N-terminal cysteine-rich domain (DKK_N) and a C-terminal collipase fold (DKK_C). DKK3 deviates from the other DKK family members in that this protein contains an additional soggy domain that is also found in dickkopf-like protein-1 (Cruciat and Niehrs, 2013).

DKK proteins $-1,-2$, and -4 can bind to LRP5/6 coreceptors with nanomolar affinity, thereby acting as functional antagonists of $\mathrm{WNT} / \beta$-catenin signaling (Joiner et al., 2013). As described above, the extracellular part of LRP5/6 consists of two pairs of $\beta$-propellers connected by EGF-like domains. Crystallization studies revealed that the DKK1_C domain binds to LRP6 $\beta$-propeller pair located closest to the plasma membrane. Unexpectedly, however, the complex appeared to consist of an equimolar mixture of a bound LRP6 inner propeller pair-DKK1_C complex and an unbound LRP6 inner propeller pair, a finding that has not yet been explained. The DKK1_N binds to the outer LRP6 $\beta$-propeller pair at the site where interaction with most of the WNT proteins takes place (Ahn et al., 2011).

Apart from binding to LRP5/6, DKK1 and -2 have been shown to bind to Kremen receptors 1 and 2 with high affinity. The interaction between LRP6, DKK, and Kremen results in the rapid endocytosis of the complex from the plasma membrane, thereby providing an additional mechanism for the inhibition of WNT signaling (Mao et al., 2002).

\section{E. Micro-RNAs}

Micro-RNAs (miRNAs) are short noncoding RNA molecules that can regulate the translation of many genes by controlling mRNA stability. Most miRNAs are encoded by genomic regions distant from annotated genes but some are located in introns of annotated genes. The primary transcripts are subsequently processed by Drosha and Dicer to form the typical double-stranded RNA strands with a length of 20-25 nucleotides. One of these strands associates with the RNA-induced silencing complex to interact with the target mRNA. This interaction is initiated by the seed sequence, a stretch of 6-8 nucleotides located at the $5^{\prime}$ terminus of the miRNA and stabilized by the anchor sequence at the $3^{\prime}$-side of the miRNA. It is now increasingly clear that the translation of multiple genes can be regulated by a single miRNA, but on the other hand, single target mRNA can have multiple miRNA binding sites (Song et al., 2015). Over the last years an overwhelming number of studies have been published where the role of miRNA regulation in a variety of diseases and signaling pathways, including WNT signaling, is reported (Anton et al., 2011).

The expression of virtually all components of the $\mathrm{WNT} / \beta$-catenin signal transduction pathway are under the control of at least one, but usually many more, miRNAs. This is also true for many of the inhibitors, including sFRPs, DKK proteins, and WIF. The result is a highly dynamic posttranscriptional regulation of the pathway, which may contribute to diseases such as cancer. A comprehensive overview of the many miRNAs regulating the different components of WNT signaling is beyond the scope of this review so we refer to a recent publication by Peng et al. (2017), where the crosstalk between miRNAs WNT/ $\beta$-catenin signaling is extensively discussed. Here we will focus on the miRNAs that have been reported to regulate WNT components in a cardiovascular context.

Regulation of WNT signaling by miRNAs has been shown to affect cardiac physiology in several studies. As an example, overexpression of miR-19b targets the expression of ctnnb1, the gene encoding $\beta$-catenin, thereby disturbing the left-right symmetry and the 
development of the heart in zebrafish embryos (Li et al., 2014a). Both miR-218 and miR-499 have been shown to affect cardiomyocyte differentiation of cardiac and mesenchymal stem cells via modulation of the expression of sFRP2 and $\beta$-catenin, respectively (Zhang et al., 2012; Wang et al., 2016b). miRNAs can also modulate the activation of cardiac fibroblasts. This was recently shown for miR-154, which, by suppressing the DKK2 expression, activated WNT signaling (Sun et al., 2016). Moreover, the stability of atherosclerotic lesions was found to be enhanced by miR-210, targeting the expression of the APC gene and thereby regulating smooth muscle cell differentiation and survival (Eken et al., 2017). It is highly likely that these studies just form the starting point for a multitude of investigations where the modulation of WNT signaling via miRNAs will be further explored in a cardiovascular context.

\section{Signal Transduction}

\section{A. Disheveled}

The DVL proteins form an essential component in the transduction of WNT signaling from the receptor level to the intracellular compartment. DVL acts as a scaffold for the formation of the WNT/FZD receptor complex. The protein was originally described as a segment polarity gene required for wingless signaling in Drosophila (Klingensmith et al., 1994; Noordermeer et al., 1994) where disheveled mutants show a phenotype similar to the wingless mutant (Nusslein-Volhard and Wieschaus, 1980). Three DVL homologs have been identified in vertebrates, but the domain structure of the protein is highly conserved. At the $\mathrm{N}$-terminal side of the protein, a DIX (disheveled-axin) domain that, as the name implies, is involved in the binding of the axin protein. The DIX domain is involved in the transduction of WNT/ $\beta$-catenin signals, where a complex of DVL, FZD, axin, and LRP5/6 is formed. In the central part of the DVL protein, there is a PDZ domain where the interaction with the KTxxxW motif of FZD proteins takes place. At the C-terminal side of the protein the DEP (disheveled-EGL10-plekstrin) domain is located. This domain can interact with a bipartite motif in the third intracellular loop of FZD and forms electrostatic interactions with membrane lipids. Recently, the DEP domain was shown to undergo a conformational change, followed by its dimerization, to trigger the formation of the WNT/FZD signalosome (Gammons et al., 2016). All DVL proteins are heavily phosphorylated and, although the mechanisms leading to this modification are still obscure, some phosphorylation sites have been shown to be necessary for DVL function (Dijksterhuis et al., 2014; Mlodzik, 2016).

Targeted mutations of the $D v l$ genes in mice give rise to relatively mild phenotypes. The Dvl1 knockout mouse shows neuronal and social behavioral abnormalities (Lijam et al., 1997). Dvl2 and Dvl3 null mutants show cardiac outflow tract defects, including double outlet right ventricle, transposition of the great arteries, or persistent truncus arteriosus (Hamblet et al., 2002; Etheridge et al., 2008). All these abnormalities can be attributed to defects in PCP signaling, whereas DVL proteins show a high level of redundancy for $\mathrm{WNT} / \beta$-catenin signaling: the presence of only a single allele of one of the three $D v l$ genes is sufficient for $\beta$-catenin-mediated signaling in mice (Wynshaw-Boris, 2012).

Next to their role in WNT/ $\beta$-catenin and WNT-PCP signaling, several other cellular functions have been described for DVL proteins, including nuclear functions, localization and/or anchoring of ciliary basal bodies in multiciliated cells, inhibition of Notch signaling, and a WNT-independent role in cell viability (Mlodzik, 2016). The relevance of these mechanisms, compared with the role of DVL in WNT signaling, still needs to be elucidated.

\section{B. WNT/ $\beta$-Catenin Signaling}

1. Characteristics of $\beta$-Catenin. $\beta$-Catenin is a central molecule in the $\mathrm{WNT} / \beta$-catenin signaling pathway. $\beta$-Catenin is protein of $\sim 80 \mathrm{kDa}$ that is present throughout the animal kingdom. The characteristics of this multifunctional protein have been extensively described in a review by Valenta et al. (2012), so we will only provide a brief summary here. $\beta$-Catenin has two rather diverse functions in the cell: on the one hand, it has a structural role as part of the cadherin-catenin cell adhesion complex, whereas on the other hand it acts as a modulator of gene transcription. It has a highly conserved structure with 12 so-called Armadillo repeats (Armadillo is the Drosophila homolog of $\beta$-catenin) in the middle, flanked by $\mathrm{N}$-terminal and C-terminal domains. The Armadillo repeats form a positively charged groove where many partners, including cadherins, APC protein, and transcription factors from the TCF/LEF family, can bind. Extensive mutation analyses have demonstrated that the N-terminal side of the Armadillo domain supports hetrodimerization with $\alpha$-catenin and subsequent formation of the cadherin-catenin complex, whereas the region C-terminal from the Armadillo domain is involved in the binding of transcription coactivators. There are multiple phosphorylation sites present in $\beta$-catenin, allowing the fine tuning of its function and degradation. (Valenta et al., 2012).

The $\beta$-catenin-dependent signal transduction pathway is unusual in that activation of WNT/FZD signaling inhibits the degradation of the intracellular signaling protein $\beta$-catenin, leading to a higher concentration of $\beta$-catenin inside the cell. This is in contrast to most GPCRs, the activation of which stimulates the intracellular production of a second messenger rather than its degradation. This $\beta$-catenin subsequently can either move to the cell adhesion complex or migrate to the 
nucleus where it can activate gene transcription (Fig. 4). At present it is not clear how $\beta$-catenins distribute over the nucleus and cell adhesion complexes. In contrast to the nuclear $\beta$-catenin pool, which has a half-life of minutes, $\beta$-catenin that is incorporated in cell adhesion complexes appears to be very stable (Clevers and Nusse, 2012). This rapid turnover of $\beta$-catenin in the nucleus may create a concentration gradient with the cytoplasm, favoring the transport to the nucleus, where $\beta$-catenin can be bound to a transcription complex, as will be discussed in detail below.

2. The $\beta$-Catenin Destruction Complex. In the absence of WNT signaling, a $\beta$-catenin destruction complex is formed in the cytoplasm consisting of axin, APC protein, glycogen synthase kinase $3 \beta$ (GSK3 $\beta$ ), casein kinase $1 \alpha(\mathrm{CK} 1 \alpha)$, and $\beta$-catenin. The axin protein contains distinct binding domains for these constituents of the complex and therefore acts as a scaffold protein. Upon the formation of the complex, sequential phosphorylation of $\beta$-catenin takes place. The first phosphorylation is at $\operatorname{Ser}^{45}$ by CK1 $\alpha$, followed by phosphorylation of $\mathrm{Thr}^{41}$, Ser ${ }^{37}$, and $\mathrm{Ser}^{33}$ by GSK3 $\beta$. Phosphorylated $\beta$-catenin is released from the complex and bound by the E3 ubiquitin ligase $\beta$-Trcp (also called TRCP-1) for ubiquitination at the $\mathrm{N}$-terminal end of the protein and subsequent degradation. This results in a lowering of the cytoplasmic $\beta$-catenin content. axin and APC can also be phosphorylated by GSK3 $\beta$ and CK $1 \alpha$, leading to enhanced $\beta$-catenin phosphorylation. The phosphorylation mechanisms are counteracted by two phosphatases, PP1 and PP2A, which can dephosphorylate axin and $\beta$-catenin, respectively, resulting in an increase in the amount of cytoplasmic $\beta$-catenin (MacDonald et al., 2009).
3. Activation of WNT/ $\beta$-Catenin Signaling. Activation of $\mathrm{WNT} / \beta$-catenin signaling results in the formation of a receptor complex consisting of WNT, FZD, and LRP5/6 at the plasma membrane. DVL is recruited to this receptor complex and, subsequently, axin, GSK3 $\beta$, and CK1 migrate from the destruction complex toward the plasma membrane. The two kinases phosphorylate each of the five PPPSPxS motifs present on the intracellular tail of LRP5/6, creating a docking site for axin and thereby promoting WNT signaling (Wolf et al., 2008). Opposite to the phosphorylation of $\beta$-catenin, here GSK3 $\beta$ appears to be priming kinase as CK1 can only phosphorylate PPPSPxS motivs previously phosphorylated by GSK3 $\beta$ (Niehrs and Shen, 2010). The migration of axin to the receptor complex at the plasma membrane results in a disruption of the $\beta$-catenin destruction complex, leading to an accumulation of the intracellular signaling protein in the cytoplasm (MacDonald et al., 2009). In the meantime, several other mechanisms have been described by which activation of WNT signaling leads to cytoplasmic accumulation of $\beta$-catenin. These mechanisms include the WNT-induced degradation of axin, direct inhibition of GSK3 $\beta$ through the phosphorylated cytoplasmic tail of LRP6, and internalization of the signalosome into multivesicular bodies, which shields the cytoplasmic $\beta$-catenin from GSK3 $\beta$ by the vesicular membrane (MacDonald and He, 2012). Moreover, tankyrase is an enzyme that positively regulates $\mathrm{WNT} / \beta$-catenin signaling by attaching a poly-ADPribose chain to axin, thereby targeting this scaffold molecule for degradation (Guettler, 2016). At present it is unclear what the individual contributions of these different mechanisms

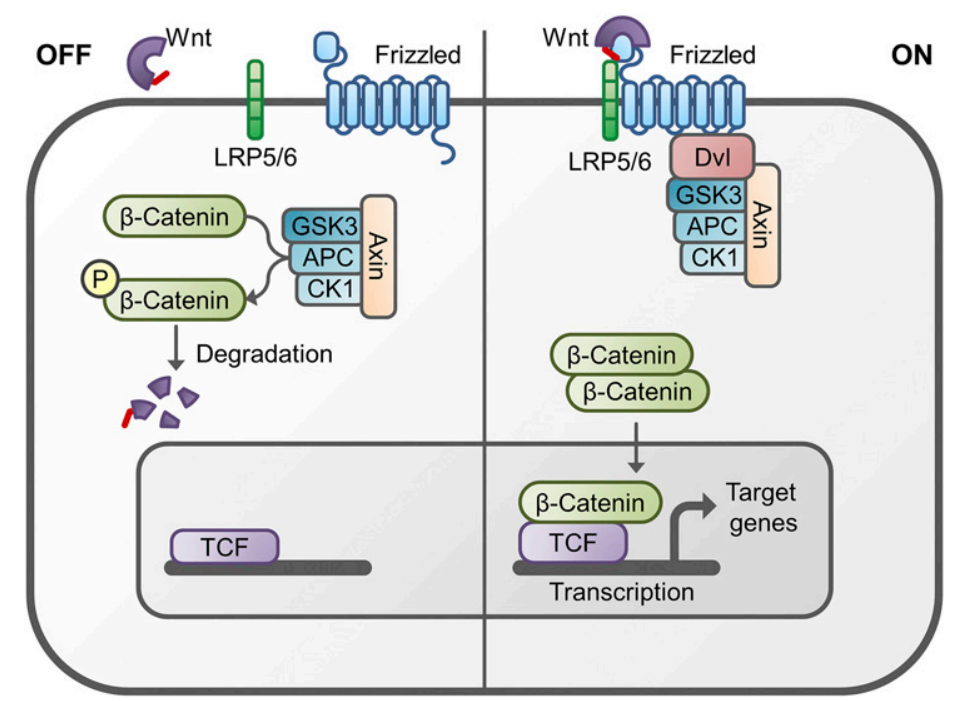

Fig. 4. Schematic representation of the WNT/ $\beta$-catenin signal transduction pathway. (Left) In the "Off" state, $\beta$-catenin is bound in a so-called $\beta$-catenin destruction complex containing glycogen synthase kinase $3 \beta$ (GSK3 $\beta$ ), axin, adenomatous polyposis coli (APC) and casein kinase-1 (CK-1). The kinases in this complex phosphorylate $\beta$-catenin, thereby targeting it for degradation by the ubiquitin proteasome system. (Right) In the "On" state, the receptor complex consisting of frizzled and LRP5/6 bind WNT, which recruits the disheveled (DVL) protein to the plasma membrane. Subsequently, several components of the $\beta$-catenin destruction complex are recruited to the membrane, which prevents the phosphorylation of $\beta$-catenin. Therefore, this protein can now accumulate in the cytoplasm and translocate to the nucleus to associate with transcription factors and stimulate the transcription of WNT target genes such as cycline-D1, c-myc, and axin2. 
are to the accumulation of cytoplasmic $\beta$-catenin upon WNT stimulation.

4. Nuclear Translocation of $\beta$-Catenin and Gene Expression. The accumulated cytoplasmic $\beta$-catenin can translocate to the nucleus, where it can activate transcription of WNT-responsive genes (Clevers and Nusse, 2012). Because the nucleus is surrounded by a membrane that is poorly permeable for large proteins, it contains multiple pores formed by nuclear core complexes. Most proteins require the interaction with specific nuclear importing or exporting proteins that act as chaperones during the nuclear membrane transport. The nuclear membrane transport of $\beta$-catenin, however, appears to be unusual because it can migrate over the nuclear membrane by a direct interaction with the nuclear core complex (Jamieson et al., 2014). Recently, interaction of hydrophobic patches in the tail region of $\beta$-catenin with the nuclear pore complex has been reported, which may explain this specific interaction (Sharma et al., 2016).

The $\beta$-catenin protein does not contain a DNA binding domain. This implies that for regulation of gene expression, $\beta$-catenin needs to form a complex with transcription factors to bind to WNT-responsive elements in the promoter region of its target genes. $\beta$-Catenin can form a complex with members of the TCF/LEF family of transcription factors that bind to specific DNA sequences, which are conserved from Drosophila to man (Molenaar et al., 1996; van de Wetering et al., 1997). In the absence of $\beta$-catenin, TCF is bound to the transcriptional repressor Groucho, which effectively silences the TCF-mediated gene expression. $\beta$-Catenin is capable of replacing Groucho, which is the first step in the formation of a transcription complex In mammals, four TCF homologs, respectively named TCF-1, -3 , and -4 and LEF-1, are present. The $\beta$-catenin/LEF-1 complex typically activates gene expression, whereas the $\beta$-catenin/TCF-3 complex acts as a repressor. The binding of TCF/LEF transcription factors involves the central region of the $\beta$-catenin protein (Arm repeats $3-10$ ). Complexes of $\beta$-catenin with TCF-1 and -4 can serve both as activators and repressors, depending on the context. Moreover, each of them have different splice variants that can affect their $\beta$-catenin binding potential. (Valenta et al., 2012).

Additional regulation of the $\beta$-catenin/TCF complex is provided by several transcriptional coactivators. These coactivators can have different functions, such as modifying the chromatin structure (e.g., CBP, p300, BCL9, and Tip60), the nucleosome arrangement (e.g., SWI and ISWI), or the association of the $\beta$-catenin/TCF complex with RNA polymerase II (members of the Mediator and paf1 complex) (MacDonald et al., 2009). Most of these co-activators, however, are not specific for the $\beta$-catenin/TCF complex. In contrast, the coactivator BCL9 interacts specifically with the first Armadillo repeat of $\beta$-catenin. In association with the coactivator Pygopus, BCL9 introduces specificity to the transcription complex (Clevers and Nusse, 2012; Valenta et al., 2012). The formation of the transcription complex can activate the expression of a large number of WNT target genes that are mostly specific for certain tissues and/or developmental stages (Lustig et al., 2002). Recently, nuclear roles for of other catenin family members such as $\alpha$-catenin and P120-catenin have been proposed that may also participate in the regulatory control of WNT signaling. This suggests the existence of a network of nuclear catenins involved in gene expression control (McCrea and Gottardi, 2016).

\section{WNT/ $\mathrm{Ca}^{2+}$ Signaling.}

One of the most abundantly used second messengers is $\mathrm{Ca}^{2+}$, leading to processes as diverse as muscle contraction, activation of enzymes such as PKC, and gene transcription. $\mathrm{Ca}^{2+}$-mediated signal transduction of FZD proteins was already reported in the $90 \mathrm{~s}$ to involve PKC and phosphatidyl inositol signaling (Cook et al., 1996; Slusarski et al., 1997). The current view on this topic is that two pathways can be employed in $\mathrm{WNT} / \mathrm{Ca}^{2+}$ signaling, one involving phospholipaseC-mediated production of inositol triphosphate and diacylglycerol and the other via the cyclic GMPselective phosphodiesterase and p38-MAPK (Ma and Wang, 2007). It is generally accepted that for both pathways the interaction between FZD proteins and heterotrimeric $\mathrm{G}$ proteins is needed, more specifically the $\mathrm{G} \alpha_{\mathrm{i} / \mathrm{o}}$ and the $\mathrm{G} \alpha_{\mathrm{t}}$ subfamilies; for further details, refer to section V.E of this review. At present it is not conclusively established whether DVL is essential for the activation of WNT/Ca ${ }^{2+}$ signaling (Schulte, 2010).

\section{WNT/Planar Cell Polarity Signaling}

During development, cell proliferation and differentiation are required to make the embryo grow and develop different organs and tissues. However, another essential mechanism is the transfer of directional information to form specific structures and to allow organs and/or tissues to grow in their final shape. Planar cell polarity (PCP) was identified as a mechanism that is involved in the generation of both global and local directional information. Examples are the morphologic changes in mesenchymal cells but also the apical/basal polarization of epithelial cells (Yang and Mlodzik, 2015). PCP was first demonstrated in Drosophila where cuticular structures such as bristles and wing hairs show a clear polarization (Adler, 2012). In vertebrates, PCP signaling regulates anterior-posterior body axis elongation during gastrulation through convergent extension and is also involved in neural tube formation. PCP signaling is also necessary for the orientation of sensory hair cells in the inner ear, as well as the orientation of hair follicles in mouse skin and cilia in airway epithelium (Yang and Mlodzik, 2015). Although less well understood than in Drosophila, the importance of PCP in vertebrate development is 
stressed by defects in cardiac outflow tracts and incomplete closure of the neural tube as observed in spina bifida, which are caused by inadequate PCP signaling. Mutations in WNT/PCP signaling components were reported for both conditions (Phillips et al., 2005; Kibar et al., 2007).

Six core factors have been identified that interact with each other in Drosophila PCP signaling: the 7TM receptor protein FZD, the 4TM protein Van Gogh/striabismus, the cadherin Flamingo, and the cytoplasmic factors DVL, Prickle, and Diego. The vertebrate homologs of these proteins are the transmembrane proteins FZD, Van Gogh-like 1 and -2 (Vang1 and -2) and Celsr, and the cytoplasmic proteins DVL, Prickle, and Inversin/Diversin. As illustrated in Fig. 5, these core PCP proteins form two complexes on the opposite sides of each cell: on one side, FZD forms a complex with DVL, Inversin/Diversin, and Celsr, whereas at the opposing side the complex consists of Vangl1/2, Prickle, and Celsr. The intracellular components of both complexes are mutually inhibitory, supporting their spatial separation at two sides of the cell. However, interaction of the complexes from two neighboring cells appears to have a stabilizing effect of the complexes where the extracellular structures of Vang1 interact with the extracellular structures of FZD. Gene dosage effects have learned that either too much or too little of a PCP core factor has detrimental effects on tissue polarity (Yang and Mlodzik, 2015).

The interaction of these core PCP components can activate several signaling pathways. The FZD/DVL complex can activate the FZD/DVL PCP pathway via the PDZ and DIX domains of DVL, which can induce cytoskeletal reorganization but can also modulate gene transcription. The cytoskeletal reorganization is linked to the activation of small GTPases (such as Rho, Rac, and Cdc42) and Rho-associated kinase. Rho-associated kinase can mediate the cytoskeletal rearrangements, which are characteristic for planar polarity signaling. On the other hand, PCP signaling can also induce a transcriptional response via JNK/p38-type MAP kinase signaling (Yang and Mlodzik, 2015).

The role for WNT in the PCP pathway is only beginning to be elucidated. In Drosophila, PCP axes are directed toward the prospective margins that are a source of WNT. In this model WNT proteins are thought to modulate the extracellular Vangl1-FZD interactions, thereby disturbing the balance between the signaling of the Vang1/Prickle/Celsr complex on the one hand and the FZD/DVL/Diego/Celsr complex on the other hand. In this model, WNTs are instructive regulators of the FZD/Vang1 polarization (Wu et al., 2013). However, it has to be noted that most of these studies have been carried out in Drosophila; for vertebrates additional research will be needed to fully elucidate the exact role of WNT in PCP signaling. Complicating factors are the multiple vertebrate WNTs, only two of which (WNT5A and -11) show selectivity for PCP signaling. Moreover, PCP signaling does not make use of LRP5/6; several PCP-specific coreceptors have been identified in vertebrates such as ROR1/2 and Ryk, tyrosine kinase receptors that may serve in the relay of the signal by phosphorylating DVL.

\section{$E$. Involvement of Heterotrimeric G Proteins in WNT/FZD Signaling}

Despite their seven-transmembrane spanning structure, FZD proteins deviate sufficiently from regular G protein-coupled receptors (GPCRs) to form a distinct class of receptors (Schulte, 2010). At present, it is not entirely clear what these structural differences mean for the interaction with components

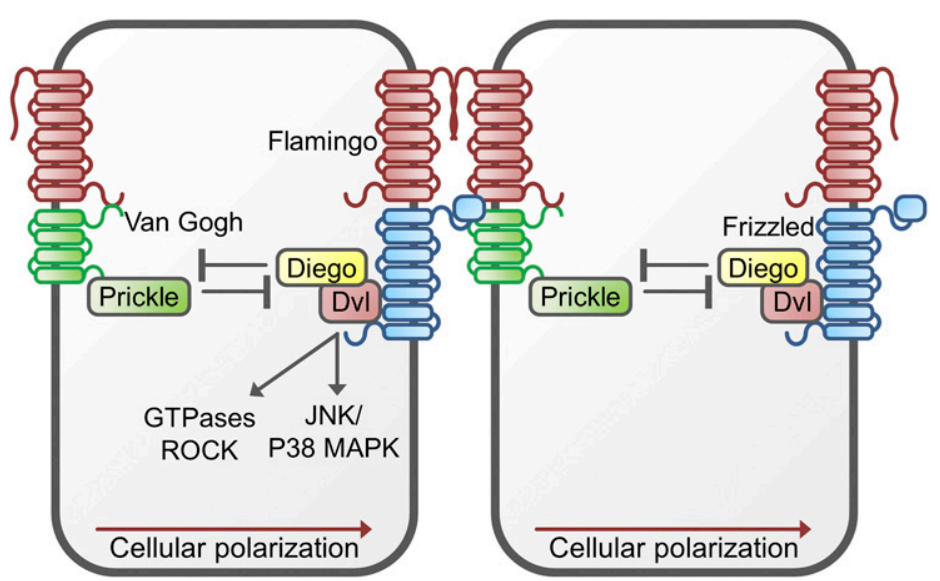

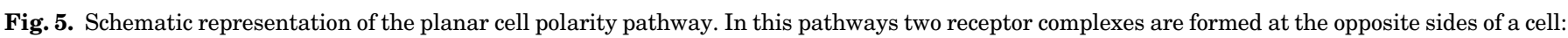

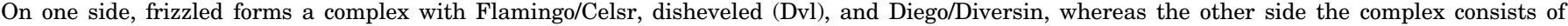

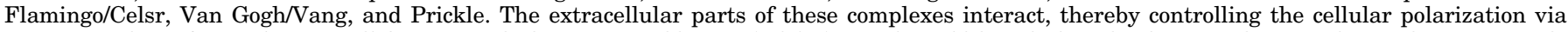

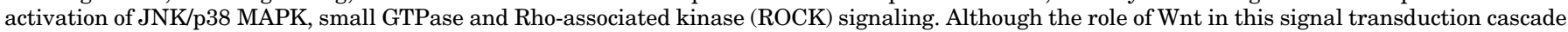

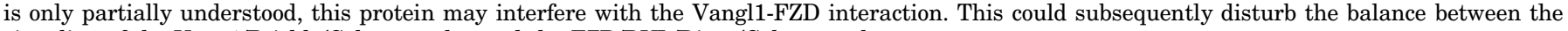
signaling of the Vang1/Prickle/Celsr complex and the FZD/DVL/Diego/Celsr complex. 
of different signal transduction pathways. Although the most prominent differences between FZD proteins and regular GPCRs are located at the extracellular side of the receptor, where FZD proteins show a bulky and highly conserved CRD, intracellular structures, particularly the third intracellular loop and the C-terminal end, also deviate. Because these intracellular domains form the point of interaction between regular GPCRs and G proteins, there is an ongoing debate whether FZD proteins can actually interact with $G$ proteins and what the consequences of this interaction would be (Dijksterhuis et al., 2014). As mentioned before, the strongest evidence for FZD-G protein interaction is available for the $\mathrm{WNT} / \mathrm{Ca}^{2+}$ signaling route, where interactions with $\mathrm{G} \alpha_{\mathrm{i} / \mathrm{o}}$ and the $\mathrm{G} \alpha_{\mathrm{t}}$ subfamilies are well established (Schulte, 2010). However, there is accumulating evidence for G protein involvement in $\mathrm{WNT} / \beta$-catenin signaling, e.g., in mouse primary microglia cells where pertussis toxin (an inhibitor of $\mathrm{G} \alpha_{\mathrm{i} / \mathrm{o}}$ ) was shown to block specific events such as phosphorylation of LRP6 and stabilization of $\beta$-catenin (Halleskog and Schulte, 2013). Moreover, interactions of the $\mathrm{G} \alpha_{\mathrm{o}}$ subunit with axin and its $\beta \gamma$-subunit with DVL have been demonstrated (Egger-Adam and Katanaev, 2010). An unresolved issue in this context is that there is overlap between the predicted binding sites for DVL and the G protein at the intracellular side of the FZD protein, and because interaction with DVL is generally accepted to be essential for $\mathrm{WNT} / \beta$-catenin signaling, it is not quite clear how the interaction with the $\mathrm{G}$ protein actually will take place (Schulte, 2015).

\section{WNT Signaling in Cardiac and Vascular Development}

The cardiovascular system is one of the first functional systems to form during embryogenesis. Both $\beta$-catenin and non- $\beta$-catenin-mediated WNT signaling play a critical role in cardiovascular development. Formation of the heart is a multistep process that starts with the specification of the cardiac progenitor cells, formation of a heart tube, with subsequent cardiac looping, chamber formation, septation, and maturation. WNT proteins, which regulate processes such as proliferation, migration, and differentiation during embryonic development and in adults, are intrinsically involved in cardiovascular development. This chapter is meant to provide the reader with sufficient background on this subject, needed for understanding the role of Wnt signaling in cardiovascular disease. This is not intended to be a comprehensive review of the development of the cardiovascular system and the role of Wnt signaling in it. We refer the readers to some high-quality review articles providing more detailed information on this subject (Moorman and Christoffels, 2003; Dejana, 2010; Sylva et al., 2014).

\section{A. $\beta$-Catenin-Mediated}

Early during development, the mesodermal cell layer is the source of cardiac progenitor cells. The formation of the mesoderm appears to be dependent on $\mathrm{WNT} / \beta$-catenin signaling. Mice devoid of $\beta$-catenin, a key player in the WNT/ $\beta$-catenin pathway, lack mesoderm (Huelsken et al., 2000), and WNT3A deficiency is associated with attenuated expression of mesodermal markers (Liu et al., 1999), making it challenging to analyze their function during cardiogenesis. However, these findings suggest a necessary requirement of $\mathrm{WNT} / \beta$-catenin signaling during formation of mesoderm, a source of cardiac progenitor cells. The initial data on the role of WNT signaling during cardiac development in vertebrates has been seemingly confusing and demonstrated contrasting results. Because of the known role of WNT signaling in promoting cardiogenesis in Drosophila, it was assumed that similar results would apply to vertebrates (Park et al., 1996). However, the activation of the WNT/ $\beta$-catenin signaling in chicken and Xenopus embryos suppressed cardiac differentiation, and the opposite effect was seen by applying WNT inhibitors (Marvin et al., 2001; Schneider and Mercola, 2001). Also, similar results were observed in mice, where multiple ectopic hearts were seen by knocking out $\beta$-catenin in the endoderm (Lickert et al., 2002). Other studies with cell-based experiments suggest a positive role for $\mathrm{WNT} / \beta$-catenin signaling on cardiac development (Nakamura et al., 2003). However, these seemingly contradicting results can be explained by the findings that the specific effects of WNT signaling are time and concentration dependent (Naito et al., 2006; Ueno et al., 2007; Wu, 2008). Once the heart tube is formed, second heart field (SHF) cells, a group of Isl1 and Nkx2.5 expressing cells, migrate to the developing heart from the dorsal pericardial wall (Buckingham et al., 2005). These highly proliferative cells form the second heart field and are tightly regulated by Wnt2 via the $\beta$-catenin pathway (Tian et al., 2010; Norden and Kispert, 2012; Ruiz-Villalba et al., 2016). Removal of the $\beta$-catenin resulted in a reduction of cells within SHF with subsequent aberrant development of the right ventricle and outflow tract (Klaus et al., 2007; Pahnke et al., 2016).

\section{$B$. Involvement of Non- $\beta$-Catenin-Mediated WNT Signaling}

Non- $\beta$-catenin-mediated WNT signaling, although less well studied, is also known to play an important role in cardiac development. WNT11 appears to be required for proper heart development by displaying cardiac promoting activity, and these effects are mainly though to be mediated by non- $\beta$-catenin-mediated signaling 
Foulquier et al.

branches (Pandur et al., 2002). Overexpression of Wnt11 in Xenopus explants induces the formation of cells with a contractile phenotype. This Wnt11 promoting activity has been shown in other models as well, such as murine embryonic stem cells, bone marrow-derived stem cells, and endothelial progenitor cells (Terami et al., 2004; Belema Bedada et al., 2005; Koyanagi et al., 2005). Furthermore, these analyses have shown that the Wnt11 procardiogenic effects are impaired by inhibiting JNK or PKC and stimulated by their activation.

WNT5A, another member of the WNT family, promotes troponin-positive cells through non- $\beta$-cateninmediated signaling in synergy with BMP6 and sFRP1 (Chen et al., 2008). However, treatment with either factor did not result in cardiomyocyte formation, which suggests that WNT5A acts in a supportive role but is not required for cardiogenesis. In contrast to WNT5A, WNT2B is required for cardiogenesis. (Wang et al., 2007). WNT2B-deficient embryonic stem cells have a significantly impaired ability to form cardiogenic progenitor cells but not hematopoietic progenitor cells. The loss of WNT2B leads to an accelerated expression of Bry and Flk1. Interestingly, WNT/ $\beta$-catenin signaling also plays a positive role in Bry expression. In addition, activation of $\mathrm{WNT} / \beta$-catenin signaling during differentiation of embryonic stem cells leads to enhanced differentiation of these cells (Anton et al., 2007). Furthermore, the loss of WNT2B function is very similar to the WNT3A function; it suggests that WNT2B might activate non- $\beta$-catenin-mediated WNT signaling. Terminal differentiation of cardiomyocytes in mouse embryonic cells is associated with a significant increase of Wnt11 expression and adding WNT11 to cultured embryonic stem cells causes an increase of beating cells (Naito et al., 2006). In Xenopus, two Wnt11 homologs have been identified, and one of them, called Wnt11A (also called Wnt11R), is expressed later (Garriock et al., 2005). Reduction of Wnt11R leads to a decrease of marker genes for cardiac terminal differentiation, and it is a cell adhesion molecule DM-GRASP that could be a non- $\beta$-catenin-mediated WNT pathway target gene (Gessert et al., 2008).

Based on the data on the role of WNT signaling during cardiac specification, the $\beta$-catenin-dependent WNT signaling pathway is required for mesoderm specification, and subsequently this pathway needs to be downregulated for formation of multipotent cardiovascular progenitor cells. This critical step is supported by the activation of the non- $\beta$-catenin-mediated WNT pathway. In the next step, the $\beta$-catenin-mediated WNT pathway is necessary for the proliferation of these cardiac progenitor cells, and finally, for the terminal differentiation, it is the non- $\beta$-catenin-mediated WNT signaling that is activated and the $\beta$-catenin-dependent WNT signaling that is downregulated (Gessert and Kuhl, 2010). After heart tube formation, the noncanonical pathway of WNT5A and Wnt11 appears to be critical in the SHF cells migration to the arterial pole of the heart (Cohen et al., 2012).

\section{Formation of Outflow Tract, Valves, and Conduction System}

Outflow tract formation and septation is intrinsically linked to cardiac neural crest cells, which are a subpopulation of cranial neural crest cells. These cells originate at the dorsal neural tube and migrate along the aortic arch arteries through the pharyngeal arches 3,4 , and 6 . The development of cardiac neural crest cells as well at outflow tract appears to be significantly dependent on the WNT signaling pathways. These crest cells express WNT1 and WNT3A and loss of $\beta$-catenin on WNT1-positive cells leads to reduction of cardiac neural crest cells as well as outflow hypoplasia in mice (Brault et al., 2001). In addition, WNT5A and WNT 11 appear to be expressed in the outflow tract myocardium. Loss of WNT5A leads to septation defects of the outflow tract, resulting in congenital heart defects such as double outlet right ventricle or persistent truncus arteriosus (Schleiffarth et al., 2007). In addition, WNT11 activates a non- $\beta$-catenin-mediated signaling pathway in the outflow tract and regulates the expression of TGF $\beta-2$ (Zhou et al., 2007). However, the underlying mechanisms leading to double outlet right ventricle formation could be due aberrant second heart field components or abnormal contribution of cardiac neural crest cells to the outlfow tract (van den Hoff and Moorman, 2000). Outflow tract formation and septation is intrinsically associated with valve formation. Hence it stands to reason that WNTs participate in valve formation.

Valve formation is a delicate and complex process where cells from the atrioventricular canal, the endocardium located between the atrial and ventricular chambers, undergo an epithelial-mesenchymal transition (EMT), migrate in the space between endocardium and myocardium, proliferate, and form the so-called cardiac cushions. Subsequently, these cardiac cushions are the precursors of the tricuspid valve and mitral valve, which separate the right and the left chambers, respectively. WNT/ $\beta$-catenin signaling is active during endocardial cushion development (Gitler et al., 2003). Furthermore, loss of APC function in zebrafish, which in turn leads to an abundance of active $\beta$-catenin signaling, results in excessive endocardial cushion formation. Overexpression of APC leads to inhibition of cardiac cushion formation (Liebner et al., 2004), demonstrating that WNTs by its inhibitory effect likely exert an inhibition on cushion formation.

WNTs likely also contribute to the development of the conduction system. This system originates from the Isl1-positive progenitor cells (Sun et al., 2007). This process starts early in development, and around embryonic day 8 in mice the cardiac pacemaking region starts taking shape in the atrium, giving rise later to the 
sinoatrial node where the impulses originate. These impulses are transmitted to the rest of the conduction system, which is composed of the atrioventricular node, the His bundle, bundle branches, and as Purkinje fibers (Sun et al., 2007). WNT7A and WNT11 are upregulated in the developing conduction system, as early as embryonic day 8 (HH 34) in chicken embryo; however, there is no functional data to delineate the precise role of WNT signaling in the formation of the conduction system (Bond et al., 2003). Also, the proper patterning of the atrioventricular canal is important on timely delay the electrical impulses from the atria to the ventricles. Recent data suggests that $\beta$-catenin-dependent WNT signaling is an important regulator of this maturation process (Gillers et al., 2015). Concurrently, coronary artery formation is a critical part of the proper cardiac development and studies suggest that WNT9b plays an important role of this process via the $\beta$-catenin pathway (Zamora et al., 2007). A summary of the WNTs in cardiovascular development is shown in Table 1.

\section{Vessels: WNT Signaling and the Control of Angiogenesis}

The formation of blood vessels is under control of two physiological processes: vasculogenesis and angiogenesis. Vasculogenesis is the formation of blood vessels from the de novo production of ECs in embryos, after which angiogenesis takes place to provide new blood vessels from pre-existing ones to all organs and tissues in both physiological and pathologic conditions.

Vasculogenesis, the initial step of vasculature development, requires the differentiation of ECs from mesodermal precursors. This first step is under the control of many signaling cues, including WNT signaling. Previous studies have demonstrated a role of WNT2, WNT3A, and WNT5A in the differentiation of embryonic stem cells toward ECs (Wang et al., 2006, 2007; Yang et al., 2009). The latter seems to involve both the $\beta$-catenin and PKC $\alpha$ pathways (Yang et al., 2009). This will be covered in more detail in section IX of this review. The contribution of R-spondin-3 also seems to be important for vasculogenesis, as well as angiogenesis. A common role for $\mathrm{R}$-spondin-3 has been identified in
Xenopus embryos and in mice and human ECs, thus highlighting the highly conserved nature of this signaling (Kazanskaya et al., 2008). R-Spondin-3 promotes the differentiation process of ESC toward angioblasts and activates the $\beta$-catenin pathway, resulting in an increased vascular endothelial growth factor (VEGF) expression (crosstalk with VEGF pathway, see section $X . C$ ) (Kazanskaya et al., 2008).

Angiogenesis is a complex process following sequential steps including the induction of tip cells, sprout elongation, vascular branching, and stabilization of the newly formed vascular network. After the initial differentiation of precursors cells to ECs, the initiation of sprouting is mediated by ECs that harbor different phenotypes: tip and stalk cells. The vascular sprout is under the guidance of tip cells at the vascular front, which have a migratory behavior. They are followed by stalk cells, which are proliferative ECs important for the extension of the sprout and involved in the creation of the vascular lumen.

1. WNT Signaling Elements in Endothelial Cells. WNT proteins can regulate the function of ECs during angiogenesis either via an autocrine or paracrine fashion, depending on whether if they are generated within endothelial or nonendothelial cells, respectively. As extensively reviewed elsewhere, many WNT ligands seem to influence EC functions (WNT2, -2B, -3, -4, -5A, -7A, -8A, -9A, -9B, -10B, and -11) (Zerlin et al., 2008; Franco et al., 2009). Moreover, ECs express a large panel of WNT receptors $\left(\mathrm{FZD}_{1},-2,-4,-5,-6,-7,-8,-9\right.$, and -10 , and LRP5 and -6), but also several key elements involved in the modulation of WNT signaling (Ryk, sFRP1 and -3, DKK1 and -3) (Zerlin et al., 2008; Franco et al., 2009).

2. Influence of WNT Signaling on Tip Cells. Evidence for a role of WNT-signaling on tip cell induction and function is scarce. WLS, a key component of the WNT secretion machinery, is a cargo receptor that mobilizes WNT proteins from the Golgi to the cell surface (Bartscherer et al., 2006). The postnatal deletion of WLS in mouse ECs (Evi-ECKO) was associated with a decreased microvascular density in postnatal retina as well as in tumor tissues (Korn et al., 2014).

TABLE 1

Wnts and cardiovascular development

\begin{tabular}{|c|c|c|c|}
\hline Gene & Pathway & Role & Reference \\
\hline WNT1 & $\beta$-catenin mediated & $\begin{array}{l}\text { Cardiac neural crest cell development and outflow tract } \\
\text { development }\end{array}$ & Brault et al. (2001) \\
\hline WNT2B & non- $\beta$-catenin mediated & Inhibition of cardiac progenitor cells & Wang et al. (2007) \\
\hline \multirow[t]{2}{*}{ WNT3A } & $\beta$-catenin mediated & Mesoderm formation & Liu et al. (1999) \\
\hline & & Inhibition of cardiac progenitor cells & Marvin et al. (2001) \\
\hline WNT5A & non- $\beta$-catenin mediated & $\begin{array}{l}\text { Cardiomyocyte differentiation and outflow tract } \\
\text { septation }\end{array}$ & Schleiffarth et al. (2007) \\
\hline WNT7A & non- $\beta$-catenin mediated & Conduction system development & Bond et al. (2003) \\
\hline WNT9B & $\beta$-catenin mediated & Epicardium development and coronary artery formation & Zamora et al. (2007) \\
\hline \multirow[t]{3}{*}{ WNT11 } & non- $\beta$-catenin mediated & Cardiomyocyte differentiation & Pandur et al. (2002) \\
\hline & & Outflow tract septation & Zhou et al. (2007) \\
\hline & & Conduction system development & Bond et al. (2003) \\
\hline
\end{tabular}


Although the tip cell density at the vascular front was reduced in this transgenic model, no differences in the number of filopodia per tip cell were observed. This suggests that tip cell function is not impacted by WNT proteins (Korn et al., 2014). More recently, the MMP (matrix metalloproteinase) inhibitor Reck (reversioninducing-cysteine-rich protein with Kazal motifs) and the adhesion G protein-coupled receptor Gpr124/ADGRA2 have been identified as key players of a WNT7A/WNT7B signaling complex for brain angiogenesis and blood-brain barrier (BBB) formation (see section VI.E) (Vanhollebeke et al., 2015; Noda et al., 2016). This atypical WNT/ $\beta$-catenin signaling was shown to selectively control the function of tip cells in central nervous system (CNS) angiogenesis (Vanhollebeke et al., 2015). Furthermore, cell migration, an important feature for tip cells, has been shown to be impacted by a WNT/ $\beta$-cateninindependent pathway, via phosphorylation of APC (Dejana, 2010; Harris and Nelson, 2010). Phosphorylated APC is present in cellular extensions during cell migration and is mandatory for cell migration and the organization of the cytoskeleton (Dejana, 2010; Harris and Nelson, 2010). Indirectly, WNT signaling could also regulate the selection of endothelial tip cells via interfering with Jagged-1. Jagged-1 is a Notch ligand that regulates the selection of tip cells by interfering with the Dll4/Notch signaling from the stalk to the tip cell (Fig. 6). Jagged-1 was significantly upregulated in ECs upon $\beta$-catenin-dependent WNT signaling (Reis et al., 2012). The study, however, supported that Jagged-1 is not directly regulated by $\beta$-catenin, because this upregulation was observed only after 48 hours of WNT3A stimulation while other genes were already regulated after 18 hours (Reis et al., 2012). Moreover, upon WNT/ $\beta$-catenin stimulation, the expression of tip cell receptors (e.g., VEGFR2) was downregulated, whereas those expressed on stalk cells (e.g., VEGFR1) were upregulated, suggesting that WNT signaling rather promotes the induction of a stalk cell-like phenotype (Reis et al., 2012).

3. Influence of WNT Signaling on Stalk Cells. After the induction of tip cells, sprout elongation is mediated by stalk cells. The importance of WNT signaling for stalk cells has been highlighted by a study performed in the developing retinal vasculature of BATgal reporter mice. Indeed, a prominent $\beta$-catenindependent activity was found in stalk cells rather than in tip cells (Phng et al., 2009). Furthermore, several WNT ligands affect the proliferation of ECs either via the $\beta$-catenin-dependent or the $\beta$-catenin-independent pathways (Franco et al., 2009). WNT1, acting via the $\beta$-catenin-dependent pathway, seemed to inhibit the proliferation of human umbilical vein endothelial cells (Cheng et al., 2003). WNT3A, via both $\beta$-catenindependent and -independent WNT signaling, stimulates the proliferation and migration of human umbilical vein endothelial cells (Samarzija et al., 2009). WNT5A, known to activate the $\beta$-catenin-independent signaling in ECs and to inhibit the $\beta$-catenin-dependent WNT signaling, has been shown to enhance angiogenesis in human ECs via an increased ECs proliferation and cell survival (Masckauchan et al., 2006; Cheng et al., 2008a). Increased WNT5A expression was also associated with increased tube formation and migration. On the other hand, the inhibition of WNT signaling by sFRP1 decreases the proliferation of ECs both in vitro and in vivo (Ezan et al., 2004). Overall, WNT $\beta$-catenindependent and independent pathways seem to have opposing effects on angiogenesis. In agreement with these findings, it has been hypothesized that the antagonistic impact of the WNT/Ca ${ }^{2+}$ signaling on the $\beta$-catenin-dependent pathway may serve as a finetuning system to balance the angiogenesis process. Conflicting results were observed, however, such as the promotion of EC proliferation and the stability of tube formation after activation of the $\beta$-catenindependent pathway by WNT1 on the one hand and the inhibition of EC proliferation and tube formation by WNT5A on the other (Goodwin et al., 2007). sFRP2 has been shown to increase EC migration, to stimulate tube formation in vitro and to induce angiogenesis in a mouse matrigel plug assay, via a calcineurin/nuclear factor of activated T-cells (NFAT) pathway (Courtwright et al., 2009).

4. WNT Signaling and Stabilization and Specification of the Vascular Network. Notch signaling is well known as a key regulator of vascular density. It is suggested that WNT could be involved in the fine tuning of Notch signaling (Phng et al., 2009). WNT signaling may therefore be in part responsible for the stability of the vascular network, whether to form or retract vascular connections. It has been shown that the loss of the Notch-regulated ankyrin repeat protein (Nrarp), $L E F-1$, or endothelial Ctnnb1 caused vessel regression (Phng et al., 2009).

Shear stress itself seems to involve $\beta$-catenindependent WNT signaling for the expression of angiopoietin 2 (Li et al., 2014b). Contrary to angiopoietin 1, which is responsible for vessel maturation, migration, and survival, angiopoietin 2 is involved in cell death and the disruption of vascular networks (Fagiani and Christofori, 2013).

Vessel maturation and stabilization are also under the control of mural cells. Pericytes are crucial perivascular cells supporting capillaries. Decreased pericyte coverage of the endothelial layer can lead to vessel loss. In the context of pulmonary hypertension (PAH), pericytes have a reduced expression of $\mathrm{FZD}_{7}$ and cdc42 -both genes being required for WNT/PCP activation, which is an important mechanism for EC motility and polarity (Yuan et al., 2015). Restoration of both genes in those pericytes was associated with improved endothelial-pericyte coverage and a larger vascular network (Yuan et al., 2015). 


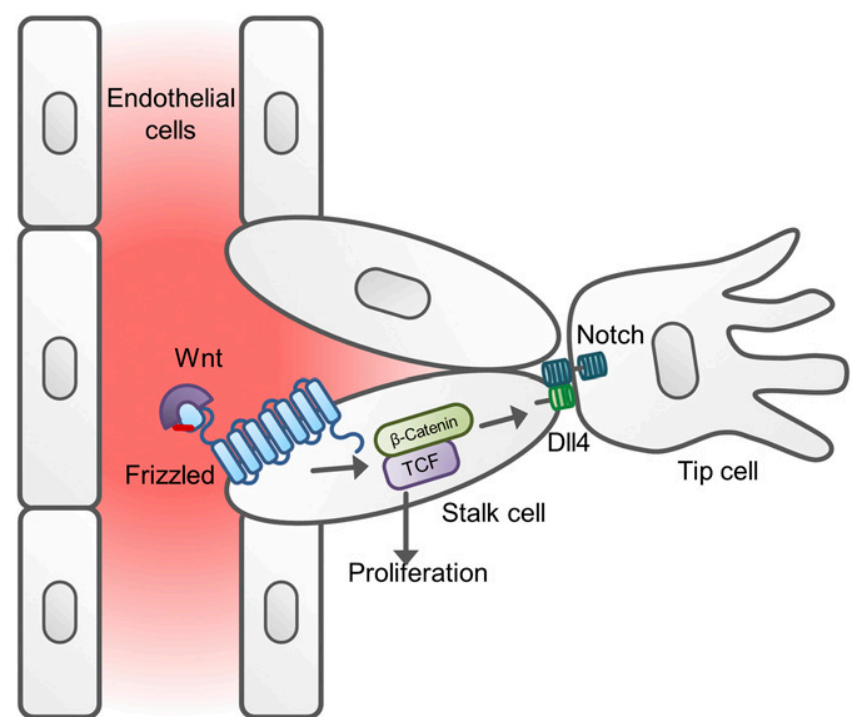

Fig. 6. The role of WNT signaling in sprouting angiogenesis. In this process, tip cells provide directional cues to the sprout cells that can proliferate and initiate the formation of a new vessel. WNT signaling can contribute to the proliferation and tube formation of the stalk cells. The effect of WNT signaling on the tip cells is likely to be indirect via the expression of delta-like ligand-4 (Dll4) in the stalk cells, which activates Notch signaling in the tip cells.

Furthermore, $\beta$-catenin-dependent Wnt signaling upregulates Sox17, a member of the SoxF family of transcription factors (Corada et al., 2014; Morini and Dejana, 2014). Sox17 activates Notch signaling via Dll4 and seems to be involved in the arterial differentiation of the vasculature. In absence of Sox17, ECs lack arterial markers and lose their ability to recruit mural cells. The upregulation of Sox 17 induced by $\beta$-catenindependent WNT signaling therefore drives ECs toward an arterial phenotype (Corada et al., 2014; Morini and Dejana, 2014).

Wnt signaling has been established as a major driver for vessel formation and maturation. It does not only control the function of the ECs but also influence their microenvironment to fine tune the angiogenic process (see also section X.C). The major hurdle limiting our understanding is the lack of temporal resolution in many studies. We expect that the use of genetic models combined with advanced in vivo imaging techniques will help to decipher the Wnt-related molecular events during the highly dynamic angiogenic process.

\section{E. WNT Signaling in the Formation of the Blood-Brain Barrier.}

The perfusion of the cerebral tissue is a critical process that needs to be strictly controlled to avoid the entrance of nondesired blood components into the CNS. This is achieved due to the unique features of the CNS ECs, which are fundamental for the establishment and maintenance of the blood-brain barrier (BBB) (Abbott et al., 2010). The BBB comprises tight junction proteins that allow a very low permeability to non-CNS components, but also specific transporters that enable the transport of nutrients (e.g., glucose, amino acids).

Supporting evidence for the contribution of WNT signaling in the development of cerebral vasculature and $\mathrm{BBB}$ has been found less than a decade ago (Liebner et al., 2008; Stenman et al., 2008; Daneman et al., 2009). The invasion of the CNS by the vasculature and the concomitant BBB development requires the presence of Wnt7A and $-B$ in the developing neuroepithelium (Stenman et al., 2008). By using the Cre-recombinase technique, the deletion of $W n t 7 A$ and $-B$ was feasible at the embryonic stage (to avoid early lethality as $W n t 7 B$ is required for placental development), and the embryos can develop up to E12.5. Subsequently these Wnt7A/B mutants die from a severe hemorrhage specifically localized to the CNS (Stenman et al., 2008). In addition, the expression of the glucose transporter (Glut1), an early BBB marker, was strongly downregulated in the Wnt7A/ $B$ mutants, suggesting the absence of a proper barrier when Wnt7A and -B are lacking (Stenman et al., 2008). The study suggested that Wnt7A and -B produced by the neuroepithelium can directly activate the ECs in the perineural vascular plexus surrounding the neural tube to promote angiogenesis and to acquire a BBB phenotype (Stenman et al., 2008). This was further confirmed in another study in which in situ hybridization experiments were performed. This study revealed that several WNT ligands are expressed by neural progenitor cells in the developing mouse brain, which could be responsible for the WNT signaling activation in ECs. Wnt7A and -B expression in particular was temporally associated with activation of $\mathrm{WNT} / \beta$-catenin pathway in several areas of the developing CNS vasculature. The impact of Wnt7A on BBB was further explored by studying the transcriptional profiles of primary brain endothelial cells using microarrays. In accordance with the findings described above, Wnt7A upregulated several BBB influx transporters, including Glut1. However, the expression of tight junction molecules was not modulated (Daneman et al., 2009). Recent studies have revealed the existence of functional interactions between WNT7A/B and Reck and Gpr124/ADGRA2, an orphan G protein-coupled receptor, in genetically engineered mice and zebrafish and luciferase reporter assays (Noda et al., 2016). Although many questions remain on the exact molecular interactions taking place between those partners, this newly discovered WNT7A/B-Gpr124-Reck complex seems important for the $\mathrm{WNT} / \beta$-catenin signaling, which is of relevance for cerebrovasculature development and BBB formation.

Further in vivo evidence was collected to confirm the major contribution of $\mathrm{WNT} / \beta$-catenin signaling for CNS vasculature development and BBB formation. First, WNT inhibition in developing embryos, using the injection of adenoviruses encoding a soluble $\mathrm{FZD}_{8}-\mathrm{FC}$ fusion protein, led to many vascular defects that were limited to the CNS (Daneman et al., 2009). Second, 
studies using reporter mice for $\mathrm{WNT} / \beta$-catenin signaling, have identified active WNT/ $\beta$-catenin signaling in ECs during brain angiogenesis, which progressively declined with the maturation of the BBB (Liebner et al., 2008; Daneman et al., 2009). In adult mice, most cerebral vessels did not exhibit $\mathrm{WNT} / \beta$-catenin activity, suggesting that a fully mature BBB does not require a permanent activation of this pathway. Furthermore, conditional activation of endothelial $\beta$-catenin in vivo was associated with an increased claudin-3 expression (a tight junction protein) and a decreased plasmalemma vesicle-associated protein expression (PLVAP, a component of endothelial fenestrations) (Liebner et al., 2008). On the contrary, the conditional inactivation of $\beta$-catenin in endothelial cells in vivo was associated with a strong upregulation of PLVAP as well as an increased $\mathrm{BBB}$ permeability. This $\mathrm{WNT} 3 \mathrm{~A} / \beta$-catenin/ claudin-3 signaling was confirmed in vitro using primary endothelial cells isolated from mouse brains. Treatment of the cells with a WNT3A-conditioned medium led to a selective increase of claudin-3 expression in association with an activation of the $\beta$-catenin pathway. WNT5A conditioned medium had no impact on the expression of claudin-3 or other tight junction proteins (Liebner et al., 2008). In addition, the upregulation of claudin-3 was dependent on the transcriptionally active form of $\beta$-catenin rather than the structural association of $\beta$-catenin with cadherins at junctional levels, thus confirming that claudin-3 is a major key effector of $\mathrm{WNT} / \beta$-catenin signaling at the BBB level (Liebner et al., 2008).

Although the contribution of $\beta$-catenin in this study was linked to an increased transcription of a tight junction protein, it is of importance to highlight the role of $\beta$-catenin (together with $\alpha$-catenin) in the composition of adherens junctions. The lack of association of $\beta$-catenin with cadherins would certainly be detrimental for BBB integrity. Unfortunately, the relative contribution of $\beta$-catenin to the adherens junctions compared with $\beta$-catenin-mediated WNT signaling in the context of BBB formation is poorly understood and should be subject to further study.

Apart from the WNT/ $\beta$-catenin pathway, a contribution of WNT signaling for BBB function through the activation of the planar cell polarity complex $(\mathrm{PCP})$ has been identified in immortalized human brain ECs (Artus et al., 2014). The PCP complex, which includes three interacting molecules, Par-3, Par-6, and an atypical PKC, is expressed in human brain ECs and colocalizes with tight junction proteins (zona occludens-1) (Artus et al., 2014). Stimulation of ECs with WNT5A was able to activate the $\mathrm{Par} / \mathrm{PKC} \alpha / \mathrm{PCP}$ complex in this cellular model. Subsequently the cellular polarization increased and the cellular permeability decreased, thus highlighting the involvement of the $\mathrm{Par} / \mathrm{PKC} \alpha / \mathrm{PCP}$ pathway to the WNT-mediated control of the BBB function.
WNT coreceptors are also crucial to control CNS vasculature. Deletion of both LRP5 and -6 in ECs severely impairs the CNS vasculature and is associated with cerebral bleeding (Zhou et al., 2014). In Lrp5 ${ }^{-1-}$ mice, there is a decreased capillary regression in the retinas, resulting especially in the retention of the hyaloid vasculature throughout life (Kato et al., 2002).

The absence of norrin (NDP, Norrie disease protein, or X-linked exudative vitreoretinopathy 2 protein) is detrimental for the regulation of angiogenesis as well as for BBB formation. Norrin can activate the WNT/ $\beta$-catenin signaling pathway by binding to $\mathrm{FZD}_{4}$ and LRP5/6. This complex has been shown to be of major importance for both blood retinal and blood-brain barriers (Wang et al., 2012). In the absence of nor$\mathrm{rin} / \mathrm{FZD}_{4}$ signaling, vascular growth in retinas is retarded and the capillary network does not form (Wang et al., 2012). In the brains of $\mathrm{Fzd}_{4}^{-/-}$mice, BBB integrity is altered in the cerebellum, olfactory bulb, spinal cord, and retina (blood-retinal barrier or BRB), but not in the cerebral cortex, striatum, or thalamus (Wang et al., 2012). Furthermore, when $\mathrm{Fzd}_{4}$ deletion was induced during adulthood, the vasculature exhibited an increased expression of PLVAP and a reduced expression of claudin-5 and this was associated with loss of BBB and BRB integrity. This highlights that $\mathrm{FZD}_{4}$ is crucial for the maintenance of mature BBB/BRB function. This conclusion has been supported by another study, in which the injection of an anti-FZD ${ }_{4}$ monoclonal antibody, blocking its function, induced a BRB dysfunction (Paes et al., 2011).

In addition to its role in $\mathrm{BBB} / \mathrm{BRB}$, norrin/ $\mathrm{FZD}_{4}$ signaling plays a central role in retinal vascular growth. Norrie disease, due to loss-of-function mutations in the Norrie disease gene (NDP, the gene coding for norrin), is associated with a severely decreased vascularization of the retina (see section VII.C) (Warden et al., 2007). Mutations downstream of the norrin pathway, in $F Z D_{4}$, LRP5, or TSPAN12 genes, are also associated with several retinopathies such as familial exudative vitreoretinopathy (see section VII.C) (Warden et al., 2007).

Although the important role played by norrin has been recognized for $\mathrm{BBB}$ formation, less is known regarding its impact in pathologic conditions. Its role was recently investigated in a rat model of subarachnoid hemorrhage (Chen et al., 2015). First, norrin expression increased over time after induction of subarachnoid hemorrhage, thus highlighting the importance of norrin signaling in this pathologic condition. Second, intracerebroventricular administration of norrin led to $\beta$-catenin nuclear translocation, increased expression levels of occludin, VE-cadherin, and zona occludens 1 , as well as a reduced $\mathrm{BBB}$ permeability shown by a reduced brain water content, a reduced Evans blue extravasation, and improved neurologic functions (Chen et al., 2015). These effects were abolished when a pretreatment with a $\mathrm{FZD}_{4}$ siRNA was 
performed. Thus, norrin protects BBB integrity in the context of subarachnoid hemorrhage by activating the $\mathrm{FZD}_{4} / \beta$-catenin pathway (Chen et al., 2015).

Taken together, these data imply that multiple WNT signaling components are important for BBB (and BRB) formation but can also play crucial roles for $\mathrm{BBB}$ maintenance in both physiologic and pathologic conditions. Although the development of drugs targeting the WNT signaling could be of major importance to seal and injured BBB, WNT-targeting compounds inducing a transient opening of the BBB on the contrary could also be of interest to allow the passage of non-BBB permeable drugs into the CNS.

\section{WNT Signaling in Vascular Disease}

\section{A. Atherosclerosis}

Atherosclerosis is a main cause of cardiovascular diseases. It is a chronic inflammatory condition that can lead to an acute clinical event due to plaque rupture and thrombosis. Although the incidence of atherosclerosis-related diseases and the associated mortality in Westernized countries have declined in the last decades, it remains a therapeutic priority (Lusis, 2000; Herrington et al., 2016). Clinical and preclinical studies have identified WNT signaling as being a key player in the development of this pathology. A schematic overview of the pathways described in this section is provided in Fig. 7 .

\section{Clinical Evidence for WNT Signaling in Atherosclerosis.}

A first clinical evidence linking WNT signaling to atherosclerosis was obtained after identifying a mutation in the LRP6 gene in a family with autosomal dominant early coronary disease, hypertension, hyperlipidemia, and osteoporosis. The functional significance of the corresponding mutation, an amino acid substitution (Cys-Arg) that affects an epidermal growth factorlike domain, was further confirmed in vitro in cells transfected with the mutant LRP6 and WNT reporter genes (Mani et al., 2007). A few years later, this 1062V LRP6 variant was demonstrated to be an independent risk factor for carotid artery stenosis in hypertensive patients. (Sarzani et al., 2011).

Next to LRP6, WNT5A has been shown to be also of importance in atherosclerotic lesions. First, WNT5A is highly expressed in the macrophage-rich regions of both human and murine atherosclerotic lesions (Christman et al., 2008). Second, WNT5A mRNA and proteins were more elevated in advanced than in less advanced arterial lesions (Bhatt and Malgor, 2014; Malgor et al., 2014). Circulating plasma WNT5A was also higher in atherosclerotic patients than in healthy controls (Malgor et al., 2014).

Furthermore, the LRP5/6 inhibitor DKK1 was also found to be increased in plasma and lesions of patients with coronary artery disease and patients with carotid plaques (Ueland et al., 2009). Similarly to the findings described above on WNT5A, DKK1 was more elevated in advanced and unstable disease. This result was confirmed in $\mathrm{ApoE}^{-/-}$mice, an experimental model for atherosclerosis (Ueland et al., 2009). Immunostaining of human material as well as in vitro experiments revealed platelets as being an important cellular source of DKK1 (Ueland et al., 2009). Further mechanistic demonstrations of the role of DKK 1 in the context of atherosclerosis are discussed below.

2. WNT Signaling and Endothelial Dysfunction. Endothelial dysfunction is a well-established hallmark of atherosclerosis development. The contribution of WNT5A to endothelial dysfunction has been highlighted recently in diabetic patients. ECs from diabetic patients had higher WNT5A expression than from nondiabetic patients and this was associated with higher levels of activated c-jun N-terminal kinase (JNK). In patients, this was correlated with lower flow-mediated dilation, an indicator of endothelial function. In vitro, inhibition of WNT5A in ECs from patients with diabetes was able to restore insulin-induced eNOS phosphorylation and NO production and this was mediated by JNK. Therefore, endothelial dysfunction in human diabetes seems to involve the WNT5A/JNK signaling pathway (Breton-Romero et al., 2016).

Stimulation of human aortic ECs by WNT5A upregulated inflammatory genes rapidly (Kim et al., 2010a). Among the different genes, COX-2 and interleukin (IL8) were strongly increased already after 1 hour of treatment (160- and 15-fold, respectively). Of particular interest, WNT5A did not upregulate COX-2 in other nonendothelial cell types, suggesting an endothelialspecific action of WNT5A. In addition, this WNT5Ainduced inflammatory gene regulation was NF- $\kappa \mathrm{B}$ dependent, as revealed by an enzyme-linked immunosorbent assay-based DNA binding assay for RelA and immunocytochemical analysis. A cytokine array further showed the enhanced expression of different cytokines, including GM-CSF, IL-1 $\alpha$, IL-3, IL-5, IL-6, IL-7, CCL2, and CCL8 at 24 hours after WNT5A stimulation compared with nonstimulated human aortic ECs. Within this experimental setting, WNT3A did not show a similar effect, suggesting a proinflammatory role of $\mathrm{WNT} 5 \mathrm{~A} / \beta$-catenin-independent signaling (Kim et al., 2010a). Further experiments with calcium chelators and protein kinase $\mathrm{C}$ inhibitor identified that the WNT5A/Ca ${ }^{2+} / \mathrm{PKC}$ pathway was responsible for endothelial inflammatory regulation (Kim et al., 2010a). The impact of WNT5A was also functionally assessed in an endothelial permeability assay. The observed enhanced endothelial permeability induced by WNT5A could translate into an early inflammatory stage of the endothelium. Moreover, recent studies focused on endothelial barrier dysfunction are pointing toward an 

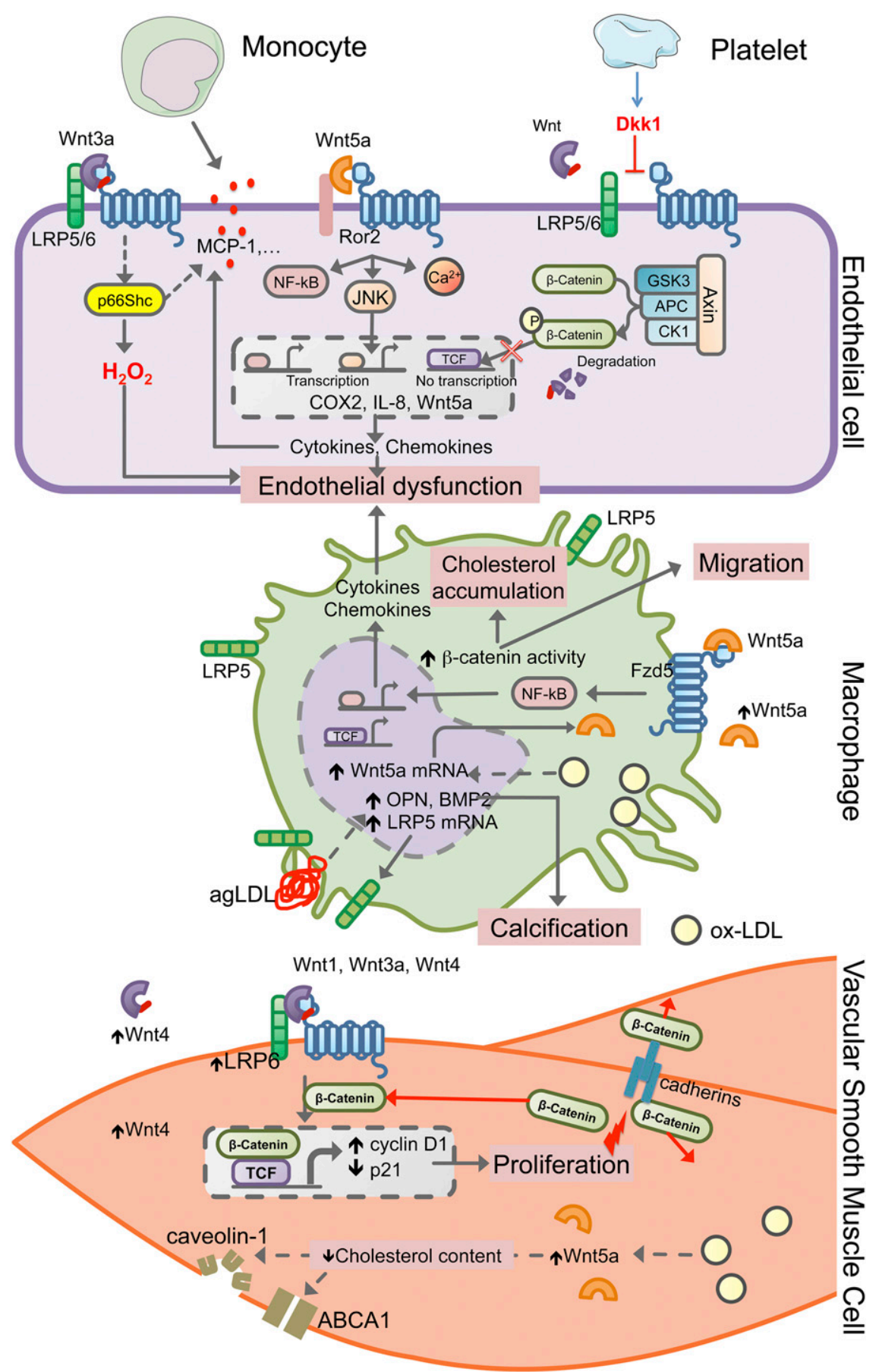

Fig. 7. WNT signaling in atherosclerosis. The contribution of WNT signaling to the development and progression of atherosclerosis is described at the intima and media levels with a central role for the contribution of monocytes/macrophages (section VII.A). ABCA1, ATP-binding cassette transporter 1; agLDL, aggregated LDL; ox-LDL, oxidized LDL; MCP-1, monocyte chemotactic protein-1.

important role of WNT5A in the regulation of endothelial permeability (Skaria et al., 2016, 2017).

As described above, DKK1 is elevated in atherosclerotic lesions and is highly expressed in platelets. During platelet activation, DKK1 is quickly released and enhances $\beta$-catenin phosphorylation in ECs. Silencing of DKK in ECs leads to an increased endothelial $\beta$-catenin phosphorylation. Furthermore, neutralizing antibodies against DKK1 abolished platelet-induced cytokine production in ECs (Ueland et al., 2009). These results suggest a deleterious role for DKK1 during atherosclerosis. Its increased release, at least in part by platelets, can inhibit the $\mathrm{WNT} / \beta$-catenin pathway in ECs and could thus contribute to endothelial dysfunction at the initiation of the atherosclerotic lesion.

The adaptor protein p66Shc is known to play a major role in endothelial dysfunction and atherosclerosis (Napoli et al., 2003; Martin-Padura et al., 2008; 
Franzeck et al., 2012; Kim et al., 2012). It was first described as an aging gene because p66Shc knockout mice have a $30 \%$ increased life span. Later studies showed that $\mathrm{p} 66 \mathrm{Sh} \mathrm{c}^{-1-}$ mice exhibited reduced oxidative stress, were protected from endothelial dysfunction during diabetes, and developed less atherosclerotic plaques (Migliaccio et al., 1999; Napoli et al., 2003; Camici et al., 2007). Vikram et al. (2014) discovered that WNT3A-stimulated dephosphorylation of $\beta$-catenin, and the subsequent $\beta$-catenin-dependent transcription was inhibited by knockdown of p66Shc. Furthermore, whereas WNT3A stimulation increased $\mathrm{H}_{2} \mathrm{O}_{2}$ levels in ECs and impaired endothelium-dependent vasodilation, p66Shc knockdown was able to rescue them both. P66Shc knockdown was also associated with an inhibition of monocyte adhesion on WNT3A-stimulated ECs.

3. WNT Signaling in Macrophages. The importance of inflammation as a hallmark of atherosclerosis has been underappreciated for many years. Since two decades, however, atherosclerosis is recognized as an inflammatory disease, in which the role of macrophages is of major importance. A dysfunctional endothelium (see above) will ultimately lead to the adhesion, rolling, and finally migration of monocytes to the subendothelial layer, where they differentiate into tissue macrophages. By scavenging modified lipoprotein particles, those macrophages become foam cells, characteristic for atheromatous lesions, and release proinflammatory cytokines, reactive oxygen species, and matrix metalloproteinases. These molecular events can lead to an amplification of the local inflammatory response and the weakening of the atherosclerotic plaque (Libby, 2002; Cochain and Zernecke, 2017).

As described in the section VII.A.1, high levels of WNT5A have been found in atherosclerotic plaques, more specifically in macrophage-rich areas (Christman et al., 2008). This finding initiated research on the role of WNT5A in the process of macrophage activation. Because the uptake of oxidized-low density lipoprotein (oxLDL) by monocytes/macrophages is playing a detrimental role in atherosclerosis, its impact on WNT5A expression has been investigated. Although native LDL had no impact, oxLDL has been found to induce WNT5A mRNA expression in human monocyte-derived macrophages (Bhatt and Malgor, 2014). By interacting with FZD $_{5}$, WNT5A induced the expression of proinflammatory cytokines. On the other hand, WNT3A had an antiinflammatory effect via suppression of GSK3 $\beta$ (Schaale et al., 2011). Both WNT3A and WNT5A have been shown to activate the NF- $\kappa \mathrm{B}$ pathway in murine and human macrophages. However, the WNT3A action was transient while WNT5A induced a more prolonged and sustained NF- $\kappa \mathrm{B}$ activation (Schaale et al., 2011).

The role of LRP5 in macrophages in the context of atherosclerosis development was investigated by Borrell-Pages et al. (2011) over the last decade.
Immunohistochemical analyses revealed the expression of LRP5 in macrophages that was increased in advanced plaques compared with initial lesions. In vitro, upon exposure to aggregated LDL, but not native LDL, human monocytes and human monocyte-derived macrophages showed a significant increase of LRP5 mRNA and protein levels and this was additionally associated with an increased $\mathrm{Wnt} / \beta$-catenin activity (Borrell-Pages et al., 2011). Cholesterol accumulation in human monocyte-derived macrophages and macrophage migration after exposure to aggregated LDL were decreased by silencing of LRP5 (Borrell-Pages et al., 2011). In addition, the expression of osteopontin and BMP2, both involved in the progression of atherosclerotic plaques, was upregulated in a LRP5-dependent manner upon aggregated LDL exposure (Dhore et al., 2001; Lund et al., 2009; Borrell-Pages et al., 2011) (see section VII.3.B). Later experiments also revealed the key role played by LRP5 to limit monocyte differentiation into macrophages (Borrell-Pages et al., 2014b). At first, immunohistochemical findings and in vitro results suggested a proatherosclerotic role for LRP5. However, the impact of LRP5 was further tested in vivo by the same authors, using Lrp5 knockout mice fed a highcholesterol diet. These mice had slightly elevated cholesterol levels, higher aortic lipid infiltration, and developed larger atherosclerotic lesions (Borrell-Pages et al. 2014, 2015a,b), similar to previous findings in $\mathrm{ApoE}^{-1-}$ mice (Magoori et al., 2003). Furthermore, the lack of LRP5 increased the transcription of LRP6, increased macrophage infiltration, and induced a proinflammatory plasma profile, thereby suggesting an atheroprotective role for LRP5 (Borrell-Pages et al., 2015b). More recently, Borrell-Pages et al. (2016a) showed that human $\mathrm{CD}^{+} 6^{+}$macrophages, which have an anti-inflammatory phenotype, had higher expression level of LRP5 than CD16 macrophages, the proinflammatory subtype (Murray and Wynn, 2011; Liu et al., 2014). It was also shown that $\mathrm{LRP}^{+}$macrophages infiltrate the deep layers of atherosclerotic plaques, suggesting increased migratory and phagocytic activities. These anti-inflammatory macrophages expressing LRP5 were abundantly present in advanced human atherosclerotic plaques, suggesting the activation of a tissue-rescue system (Borrell-Pages et al., 2016a).

The identification of therapeutic targets promoting plaque regression is of major interest. Among the different potential options, targeting plaque macrophages appears to be a relevant approach due to its importance for inflammation, lipid uptake, and plaque destabilization. For this purpose, transcriptional differences between macrophages in regressing versus progressing plaques have been studied in animal models of plaque regression (Ramsey et al., 2014). The study demonstrated that the $\mathrm{WNT} / \beta$-catenin pathway and SOX family members are activated in plaque macrophages during regression (Ramsey et al., 2014). In 
summary, WNT signaling is not only a key pathway for atherosclerosis development, but also seems to be of importance for its regression. This highlights the relevance of new therapeutic strategies targeting components of the WNT signaling pathway in the treatment of atherosclerosis.

4. WNT Signaling in Vascular Smooth Muscle Cells. Smooth muscle cells, located in the vascular media, exhibit a contractile phenotype and a low proliferation rate. During atherosclerosis development, however, endothelial dysfunction and the associated inflammatory state of the vessel wall result in the activation and de-differentiation of the vascular smooth muscle cells (VSMCs) toward a synthetic phenotype. These synthetic VSMCs subsequently migrate into the intima, proliferate, and increase the production of extracellular matrix. The thickened intima that results from this pathologic process paves the way for the development of the atherosclerotic plaque (Owens et al., 2004; Mill and George, 2012).

The impact of $\beta$-catenin on VSMC proliferation is well known. $\beta$-Catenin/TCF signaling can upregulate the expression of several proproliferative genes such as cyclin D1 and decrease the level of the cell cycle inhibitor p21 (Wang et al., 2002; Quasnichka et al., 2006). Functional evidence linking this pathway to vascular wall injury has been demonstrated in a balloon injury model (Slater et al., 2004). More recently, a study using carotid ligation in a Wnt reporter mouse has demonstrated the involvement of $\beta$-catenin signaling in both media and intima 3 and 28 days after the injury (Tsaousi et al., 2011b). Moreover, it has also been suggested that the pool of $\beta$-catenin located at the plasma membrane, involved in cell adhesion complexes, could be mobilized after disruption of the cell-cell adhesion upon migration of VSMCs from the media to the intima (Uglow et al., 2003; Slater et al., 2004).

Wnt/FZD signaling is involved in the control of VSMC proliferation. Its inhibition by FrzA, a member of the sFRP family that negatively modulates Wnt signaling, leads to antiproliferative actions both in vitro and in vivo (Ezan et al., 2004). The identification of the Wnt proteins involved in VSMC proliferation has been the focus of several studies. First of all, both WNT1 and WNT3A have been shown to induce the expression of cyclin D1 in VSMCs (Marchand et al., 2011). LRP6 could also promote VSMC proliferation. Indeed, the upregulation of LRP6 increased WNT1-induced TCF activation and the expression of a dominant negative mutant of LRP6 abolished WNT-induced survival, cyclin D1 activity, and cell cycle progression (Wang et al., 2004).

WNT4 was recently identified also to have an impact on VSMC proliferation. The mRNA expression of WNT4 was found to be elevated in proliferating VSMCs. Knockdown experiments as well as experiments using WNT4-conditioned medium confirmed the role of WNT4 in VSMC proliferation. The expression of WNT4 was also found to be elevated in injured carotid arteries: in the media at 3 days and in the intima at 28 days after injury, in line with the intimal migration of VSMCs. The significance of WNT4 expression for atherosclerosis was further evidenced by the decreased intimal lesion size and intimal cell proliferation rate found in $\mathrm{Wnt4}^{+/-}$mice (Tsaousi et al., 2011a).

Since cholesterol accumulation is of major importance for the progression of atherosclerosis, the impact of WNT5A [known to be upregulated in atherosclerotic lesions and plasma (Christman et al., 2008; Bhatt and Malgor, 2014; Malgor et al., 2014)] on this process has been investigated on VSMCs treated with oxLDL (Qin et al., 2014). WNT5A protein was increased in a concentration and time-dependent manner by oxLDL treatment. After WNT5A knockdown, total cholesterol and free cholesterol content increased signipicantly upon exposure to oxLDL (Qin et al., 2014). Reciprocally, both levels were decreased after treatment of the cells with WNT5A. This action mediated by WNT5A was associated with a regulation of $\mathrm{mRNA}$ and protein expression of caveolin-1 and ATP binding cassette transporter A1, two important players of reverse cholesterol transport (Qin et al., 2014).

A recent study investigating miRNA expression profiles in patients with stable and unstable carotid plaque has revealed miR-210 as being the most significantly downregulated miRNA in local plasma material. It was shown that miR-210 inhibits APC and thus favors Wnt signaling in VSMCs of the fibrous cap, therefore promoting their proliferation and survival (Eken et al., 2017). Further studies are required to decipher the contribution of other miRNAs in the development of atherosclerosis.

The involvement of Wnt signaling at the different stages of atherosclerosis is highlighting its importance for this pathology (Hermans and Blankesteijn, 2015; Matthijs Blankesteijn and Hermans, 2015). Recently, it was shown that inhibition of Wnt signaling by sclerostin, a protein that binds to LRP4, LRP5, and LRP6, attenuated angiotensin II (AngII)-induced aortic aneurysm and atherosclerosis (Krishna et al., 2017). This was associated with a decreased macrophage infiltration and a decreased expression of $\beta$-catenin responsive genes (Krishna et al., 2017). This supports the idea of targeting the Wnt pathway to limit or stop the progression of atherosclerotic lesions.

\section{B. Vascular Calcification}

Vascular calcification is a key process involved in the development of atherosclerosis as well as other cardiovascular pathologies such as congestive heart failure and chronic kidney disease (Demer and Tintut, 2008; Vervloet and Cozzolino, 2017). Vascular calcification has similarities with embryonic bone formation, in which WNT signaling is playing an important role (Wang et al., 2014b). 
Early studies have identified a link between Msx2, a homeodomain transcription factor involved in bone anabolism, and WNT signaling (Shao et al., 2005, 2007; Cheng et al., 2008b). Indeed, Msx2 has been found to upregulate WNT3A and WNT7A, while downregulating the expression of the $\beta$-catenin-dependent inhibitor DKK1 in primary aortic myofibroblasts (Shao et al., 2005). Further in vivo analyses with mice overexpressing Msx2 in the aorta, confirmed the suppression of aortic DKK1 and the upregulation of vascular WNT expression (Shao et al., 2005). It was thus hypothesized that Msx2 activation was acting on the calcification process in a paracrine manner by signaling via the WNT pathway. In addition, mice overexpressing Msx2 exhibited an increased bone volume compared with their wild-type littermates (Cheng et al., 2008b). In vitro investigations revealed WNT7-LRP6 signaling as being required for the induction of osteogenic differentiation of mesenchymal cells by Msx2 (Cheng et al., $2008 \mathrm{~b}$ ). The signaling cascade was further investigated at the vascular level and bone morphogenetic protein 2 (BMP2) was identified as an upstream regulator of medial artery calcification in vivo (Shao et al., 2006, 2007). Injection of BMP2 in LDLR ${ }^{-1-}$ mice was, as expected, able to increase aortic Msx2 expression, aortic WNT signaling and aortic calcium content (Shao et al., 2006). The relevance of BMP2 for vascular calcification is supported by clinical data showing an elevation of serum BMP2 levels in chronic kidney disease patients (Rong et al., 2014). Mechanistic explanations were provided by in vitro experiments using rat vascular smooth muscle cells. Stimulation of these cells with BMP2 induced cell apoptosis and upregulation of $\beta$-catenin, Msx2, and Runx2. This effect was dependent on $\beta$-catenin signaling (Rong et al., 2014). It was suggested that TNF- $\alpha$, a known activator of BMP2, could constitute a relevant stimulus of the BMP2-Msx2WNT signaling in vascular pathologies (Shao et al., 2007).

Other pathologic triggers should also be considered for the study of WNT signaling in vascular calcification. The advanced glycation end products (AGEs) and their receptors RAGEs are of particular relevance for diabetic disorders as well as other inflammatory diseases. A recent study performed in human arterial smooth muscle cells has shown that AGEs/RAGEs can promote calcification via $\mathrm{WNT} / \beta$-catenin signaling (Liu et al., 2016). It is hypothesized that RAGEs may activate genes, promoting the differentiation of arterial smooth muscle cells into osteogenic cells through the WNT/ $\beta$-catenin pathway. The contribution of WNT $/ \beta$-catenin signaling for the osteogenic transdifferentiation of vascular smooth muscle cells and calcification seems to be under the control of Runx2, a transcription factor of osteoblasts (Cai et al., 2016). Activation of the WNT/ $\beta$-catenin pathway (e.g., with WNT3A) induces the expression of Runx2, which in turn, controls key processes for osteoblast differentiation. On the contrary, blockade of WNT/ $\beta$-catenin using DKK 1 inhibits Runx2 induction and calcium depositions in vascular smooth muscle cells (Cai et al., 2016). Moreover, when bone marrow-derived mesenchymal stem cells (MSCs) are in contact with calcified vascular smooth muscle cells, they display an osteoblast phenotype. This differentiation seems to be due to an activation of the WNT5A/ROR2 pathway (Xin et al., 2013; Guan et al., 2014).

Matrix metalloproteinases (MMPs) are remodeling the extracellular matrix and are therefore suggested to play a role during vascular calcification. Their contribution has been evidenced recently in uremic vascular calcifications (Hecht et al., 2016). Furthermore, the calcification of vascular smooth muscle cells by WNT activation seemed to involve an enhanced expression of MMP-2 and MMP-9 (Freise et al., 2016). Increased expression and activity of MMPs is suggested to further promote the process of vascular calcification.

Although most findings seem to support the deleterious effect of WNT signaling on vascular calcification, a recent study identified WNT16 as being able to attenuate TGF $\beta$-induced chondrogenic transformation of vascular smooth muscle cells (Beazley et al., 2015). In this study, WNT16 was found to sustain Notch activity, which in turn maintained the contractile phenotype of vascular smooth muscle cells (Beazley et al., 2015). LRP6 was also found to limit the process of vascular calcification. Indeed, the conditional deletion of $L R P 6$ in the vascular smooth muscle cell lineage increased the aortic calcium content and decreased aortic distensibility in diabetic $\mathrm{LDLR}^{-/-}$mice (Cheng et al., 2015). The protective vascular function of LRP6 may be due to a downregulation of $\beta$-catenin-independent FZD signaling (Cheng et al., 2015).

\section{Norrie Disease}

Norrin is a retinal growth factor with angiogenic and neuroprotective properties (see section IX) (Xu et al., 2004). Norrin binds to $\mathrm{FZD}_{4}$ receptors and subsequently activates the $\mathrm{WNT} / \beta$-catenin pathway. This activity is furthermore increased in the presence of Tspan12 within the norrin/FZD $\mathrm{FZ}_{4}$ receptor complex. A defect in norrin or $\mathrm{FZD}_{4}$ has a detrimental impact on retinal vasculature during developmental angiogenesis (Ohlmann and Tamm, 2012). The so-called Norrie disease, associated with mutations in the NDP gene, causes blindness as well as progressive deafness and mental retardation (Berger et al., 1992). This also applies for familial exudative vitreoretinopathy, retinopathy of prematurity, and other retinal hypovascularization diseases (Ye et al., 2010; Ohlmann and Tamm, 2012). Next to norrin and $\mathrm{FZD}_{4}$, LRP5 and TSPAN12 have been found to be also associated with familial exudative vitreoretinopathy, thus highlighting the importance of the norrin/ $/ \mathrm{FZD}_{4}$ signaling for proper retinal 
vasculature development (Wang et al., 2012; Gilmour, 2015). The cysteine-rich domain (CRD) of $\mathrm{FZD}_{4}$ has been shown to play a critical role in norrin- $\mathrm{FZD}_{4}$ binding (Smallwood et al., 2007). Specific mutations of the FZD in the cysteine-rich domain led to a defective norrin/ $\beta$-catenin signaling in Xenopus embryos (Zhang et al., 2011).

The retinal vasculature in mice lacking norrin, $\mathrm{FZD}_{4}$ and Tspan12 is massively impaired. The superficial retinal vascular plexus is delayed and incomplete compared with WT and they all lack the intermediate and deep vascular plexi (Zuercher et al., 2012). Further analyses of the retinal vasculature development revealed that, in a norrin knockout model, the vascular density and number of branching points were decreased, whereas the number of filopodia was significantly increased (Zuercher et al., 2012). However, the morphology of the filopodial protrusions was different compared with WT with more narrow angles and a reduced amount of long tip cell protrusions (Zuercher et al., 2012). In addition, an excessive recruitment of mural cells, required to stabilize the nascent vasculature, was observed in the retinal vessels from the norrin knockout mice, suggesting a potential interaction with PDGF- $\beta$ /PDGFR- $\beta$ signaling. The overall decreased vascular density and impaired vascular growth is suggested to be related to a decreased proliferation of the retinal ECs in the norrin knockout mice. This was evidenced directly in the retina after systemic injection of bromodeoxyuridine, as well as during an in vitro assay in which norrin was identified as a mitogenic stimulus for engineered HEK293 cells (Zuercher et al., 2012).

By using mouse genetic and cell culture models, it was demonstrated that Muller glia cells are responsible for norrin production, which then stimulates $\mathrm{FZD}_{4}$ receptors located on retinal ECs (Ye et al., 2009). Subsequently, the norrin/ $\mathrm{FZD}_{4} / \mathrm{LRP} 5 / 6$ signaling is responsible for a transcriptional program in ECs to promote vascular growth and the endothelial-mural cell interactions. This program is at least partly under the control of the transcription factor Sox17. It was shown that the transfection of Sox 17 was able to rescue the ability of $\mathrm{Fzd}_{4}$ knockout retinal ECs to form capillarylike structures in a Matrigel assay (Ye et al., 2009). In addition, although $\mathrm{FZD}_{4}$ is also expressed in photoreceptors and some other retinal neurons, it was shown that the impaired visual function in $\mathrm{Fzd}_{4}$ knockout mice is linked to a defect in retinal vasculature rather than an impaired signaling in retinal neurons (Ye et al., 2009).

Finally, norrin/FZD $/ \mathrm{LRR}_{4} / 6$ signaling is also involved in other retinopathies, opening thus new avenues for therapeutic interventions. In addition, this signaling complex seems to be also of importance for other nonretinal disorders, such as female infertility (Hsieh et al., 2005; Luhmann et al., 2005).
Its contribution in this field needs to be further investigated.

\section{Pulmonary Arterial Hypertension}

Pulmonary arterial hypertension (PAH) is a lifethreatening disease associated with increased pulmonary pressures, subsequently followed by development of right-sided heart failure. The pathology is characterized by a microvascular rarefaction and an increased pulmonary vascular resistance resulting from wall thickening due to smooth muscle cell proliferation (Rabinovitch, 2012).

The relevance of WNT signaling within this context was revealed by the expression profile of lasermicrodissected pulmonary arterial resistance vessels obtained from idiopathic $\mathrm{PAH}$ and donor lung tissue (Laumanns et al., 2009). Pathway analysis demonstrated a significant upregulation of the WNT/PCP pathway. Moreover, $\beta$-catenin expression was also upregulated in ECs of pulmonary arteries from PAH patients compared with control subjects (Laumanns et al., 2009).

Thickening of the pulmonary arterial wall is observed in PAH due to an increased proliferation of vascular smooth muscle cells in the media. This has been found to be associated with higher levels of active $\beta$-catenin within pulmonary artery smooth muscle cells (PASMCs) in PAH patients (Takahashi et al., 2016). In addition, PASMCs from PAH patients also harbor higher expression levels of the platelet derived growth factor (PDGF)-BB and its receptors. PDGF-induced growth of PASMCs that turned out to be dependent of $\beta$-catenin activation and GSK3 $\beta$ inhibition (Takahashi et al., 2016). WNT5A was able to repress PDGF-BBinduced proliferation in PASMCs from healthy patients but not from PAH patients (Takahashi et al., 2016), highlighting a potential defective signaling in PASMCs during PAH.

Idiopathic $\mathrm{PAH}$ can also result from mutations in bone morphogenetic protein (BMP) receptor II (BMPRII). Such mutations are associated with apoptosis of pulmonary artery ECs as well as microvascular rarefaction during idiopathic PAH (de Jesus Perez et al., 2009). This is of particular interest as the proliferation, survival, and migration of pulmonary artery ECs induced by BMP2 is dependent of $\beta$-catenin to mediate GSK3 $\beta$ inactivation, but also dependent of Smad1 phosphorylation to recruit DVL and activate the RhoA/Rac1 pathway (de Jesus Perez et al., 2009). The relationship between the BMPRII and WNT signaling pathways has been further studied using primary cells from patients (induced pluripotent stem cell-derived mesenchymal and ECs) to identify molecular signatures of PAH (West et al., 2014). The results confirmed the increased WNT signaling in the cells of PAH patients and established a link with decreased BMPR2 signaling (West et al., 2014). In particular, expression analyses 
from PAH patient lung tissue and BMPRII mutant mice revealed an increased expression of the WNT inhibitor sFRP2 (West et al., 2014).

Finally, the progression and severity of $\mathrm{PAH}$ pathology is associated with a dysregulation of miRNA expression (Courboulin et al., 2011; Rhodes et al., 2013). Recently, a systematic investigation has been carried out via miRNA profiling of lung tissue from endstage idiopathic PAH (Wu et al., 2016a). A pathway enrichment analysis identified $\mathrm{WNT} / \beta$-catenin signaling as being the most affected. Five miRNAs (let-7a-5p, miR-26b-5p, miR-27b-3p, miR-199a-3p, and miR-656) relevant for $\mathrm{PAH}$ pathogenesis were significantly increased and correlated with hemodynamics and histopathological parameters of PAH (Wu et al., 2016a). Moreover, many WNT family genes $\left(F Z D_{4}, F Z D_{5}\right.$, CTNNB1) and downstream targets (CCDN1, VEGFA, axin2) were upregulated in end-stage idiopathic $\mathrm{PAH}$ lung tissue (Wu et al., 2016a).

In conclusion, WNT signaling pathways appear to exert a key role of the preservation of pulmonary vascular homeostasis. Future studies are required to investigate the therapeutic potential of WNT signaling for PAH (de Jesus Perez et al., 2014).

\section{E. Conclusions and Suggestions for Further Research}

Wnt signaling is not only an important key pathway for atherosclerosis development but seems to be also of importance for its regression. The activation of Wnt signaling in atherosclerosis has been firmly established, not only in experimental models but also in patients by measuring pathway components in the circulation. This activation is likely to contribute to endothelial dysfunction, the inflammatory response, and VSMC proliferation and appears to involve both $\beta$-catenin-mediated and non- $\beta$-catenin-mediated WNT signaling. Most of the studies published to date on this subject made use of genetic interventions, so a suggestion for further research would be to include pharmacological interventions. Because of the involvement of multiple signaling pathways, intervening in the WNT production and/or at the level of the receptor complex appears to be a good starting point for these experiments. In the case of vascular calcification, most of the experimental data presented so far point to activation of the $\beta$-cateninmediated signaling. Therefore, it would be interesting to study interventions in this branch of the Wnt signaling pathway. There is a rapidly growing number of drugs specifically developed for targeting $\beta$-cateninmediated WNT signaling (please refer to section XI), and these drugs appear to be interesting candidates to be tested in vitro and in animal models of vascular calcification. PAH is a condition for which new therapeutic approaches are urgently needed. The field has been hampered for a long time by the lack of animal models that adequately represent the pathologic aspects of this condition. Fortunately, new animal models for PAH have been proposed (Colvin and Yeager, 2014) that can be used to test the effects of therapeutic interventions in WNT signaling on the pulmonary vascular resistance.

\section{WNT Signaling in Cardiac Disease}

\section{A. WNT Signaling in Myocardial Infarction}

Myocardial infarction (MI) is one of the most frequent acute cardiovascular events. It usually occurs as a consequence of the progression of an atherosclerotic plaque toward an unstable phenotype, where the fibrous cap ruptures and releases the content of the plaque into the circulation. However, myocardial infarction can also be the result of plaque erosion with distal embolization or coronary vasospasm (RegitzZagrosek et al., 2016). In any case, due to the interrupted blood flow the affected region of the heart is deprived of oxygen and nutrients, resulting in cell loss in that area.

Despite the fact that stem cells have been identified in the heart, the repair of the infarct area does not result in the significant regeneration of the lost cardiac muscle (Laflamme and Murry, 2011). Instead, it shows many similarities to the wound healing process in the skin: The initial response consists of an inflammatory reaction involving polymorphonuclear neutrophils and macrophages. The function of these cells is to clear the infarct area from the necrotic debris, which is an essential first step in the repair of the infarct. The inflammatory phase is followed by the formation of granulation tissue, which typically is rich in (myo)fibroblasts and newly formed blood vessels, embedded in a loosely organized extracellular matrix. The myofibroblasts in the granulation tissue can be derived from different sources such as resident fibroblasts, circulating fibrocytes, or epicardial cells undergoing epithelialto-mesenchymal transition (EMT) (Daskalopoulos et al., 2012). Because of their unique combination of contractile and matrix synthesizing properties, the myofibroblasts play an important role in the formation of a compact and strong replacement of the lost cardiac cells (van den Borne et al., 2010) This granulation tissue gradually develops into scar tissue by losing cells and increasing the amount and the cross-linking of extracellular matrix (Hermans et al., 2016).

1. Effects of Modulation of WNT Expression and Secretion. As indicated in section VI, WNT signaling is particularly active during cardiac development. In contrast, little WNT signaling takes place in the adult heart under normal physiologic conditions. This changes during pathologic remodeling of the heart, where many fetal genes, including components of WNT signaling, become reactivated (Kuwahara and Nakao, 2011). In a mouse model of MI, where the underlying pathology of human infarction is mimicked by a surgical ligation of a coronary artery, the 
modulation of the expression of multiple genes of the WNT pathway has been reported. This includes the upregulation of WNT2, $-4,10-\mathrm{b}$, and -11 expression and the downregulation of WNT7B (Barandon et al., 2003; Aisagbonhi et al., 2011; Paik et al., 2015; Morishita et al., 2016). Moreover, in the same studies the increased expression of $\mathrm{FZD}_{1},-2,-5$, and ${ }_{-10}$ and the decreased expression of $\mathrm{FZD}_{8}$ was demonstrated (Barandon et al., 2003; Aisagbonhi et al., 2011). Recently, Mizutani et al. (2016) used in situ hybridization to monitor the expression of all 19 WNTs in a cryoinjury model in neonatal mice. They found that WNT2B and WNT5A and, to a lesser extent, WNT9a were upregulated in the injured epicardial layer. WNT3A, $-4,-5 \mathrm{~B},-6$, $-8 \mathrm{~A},-9 \mathrm{~B}$, and $-10 \mathrm{~B}$ were upregulated in the myocardium of the injured hearts, whereas WNT8B, $-10 \mathrm{~A},-11$, and -16 were similarly expressed in injured and uninjured hearts. These results demonstrate the modulation of WNT signaling in injured hearts but also highlight the potential effects of age (neonatal vs. adult) and injury model (cryoinjury vs. coronary ligation) on the expression pattern of WNT genes. For a detailed overview of the role of different WNT proteins in cardiac function, we refer the reader to an excellent review on this subject (Dawson et al., 2013).

In several studies, the effect of modulation of WNT expression or secretion on infarct healing was addressed. Applying coronary artery ligation and cryoinjury in a mouse model of cardiomyocyte-specific overexpression of WNT10B, an increase in neovascularization of the infarct zone, a smaller scar size with fewer myofibroblasts and an improved ventricular function were observed (Paik et al., 2015). Similar beneficial results were reported in a mouse model of MI, in which WNT11 was overexpressed via adeno-associated virusmediated overexpression, where a marked reduction in the inflammatory response in the infarcted heart was reported (Morishita et al., 2016). An attenuated inflammatory response and increased neovascularization could also be induced by the macrophage-specific deletion of the WNT transporter WLS (csfm-icre; Wls $s^{f l / f l}$ mouse). The WLS-deficient macrophages displayed an M2 phenotype, known to stimulate repair and angiogenesis (Palevski et al., 2017). Interference with the lipidation of WNT proteins by intravenous administration of the PORCN inhibitor GNF-6231 to a mouse model of MI also reduced infarct size, prevented adverse remodeling, and reduced the decline in cardiac function. Similar beneficial effects were observed in a recent study, in which the PORCN inhibitor WNT-974 (formerly known as LGK974) was administered by oral gavage to infarcted mice, starting at 1 week after MI induction. The investigators observed an unanticipated proregenerative response in heart muscle and a collagen-VI-dependent antifibrotic effect of the treatment and concluded that PORCN inhibition could be a promising therapeutic strategy to block pathologic remodeling in MI patients (Moon et al., 2017) On the other hand, direct injection of recombinant WNT3A protein in the border zone of the infarct area was shown to have detrimental effects on infarct healing, because this treatment was found to inhibit the proliferation of cardiac side population cells (Oikonomopoulos et al., 2011). From these studies it can be concluded that more general interventions in WNT processing and secretion result in better infarct healing, but that more studies are needed to determine the positive and negative contribution of individual WNTs to the wound healing process.

2. Modulation at the Level of Secreted FrizzledRelated Proteins. As indicated in section IV.B of this review, sFRPs are thought to act primarily as negative modulators of Wnt signaling. Barandon et al. (2003) were the first to demonstrate the upregulated expression of sFRP1 after MI. In transgenic mice overexpressing FrzA, the bovine homolog of sFRP1, these authors demonstrated the beneficial effect of FrzA on infarct size and cardiac function after MI. These beneficial effects were attributable to a reduction in cardiomyocyte apoptosis and leukocyte infiltration and an improved capillary density in the scar. Similar results were obtained when bone marrow cells of sFRP1 overexpressing mice were transplanted in wild-type recipients, which subsequently were subjected to MI (Barandon et al., 2011). A genetic variant of sFRP1 was reported to be associated with an increased risk of MI in a Chinese Han population (Tao et al., 2016). An antiinflammatory and antiapoptotic role for sFRP5 has also been described in a mouse model of ischemia/ reperfusion damage (Nakamura et al., 2016), supporting a beneficial effect of sFRPs on infarct healing.

The role of sFRP2 in infarct healing has been addressed in several publications. sFRP2 was shown to be a paracrine factor secreted from stem cells and to activate an antiapoptotic gene expression profile in cardiomyocytes (Mirotsou et al., 2007). Direct injection of recombinant sFRP2 in the infarcted myocardium of rats was reported to have antifibrotic effects and improved cardiac function (He et al., 2010). sFRP2 was also found to contribute to the engraftment of mesenchymal stem cells (MSC), and injection of sFRP2-overexpressing MSC in the border zone of infarcted mice improved the wound healing and cardiac function (Alfaro et al., 2008) On the other hand, mice lacking the sFRP2 gene also were shown to have decreased fibrosis and improved cardiac function after being subjected to MI compared with wild types (Kobayashi et al., 2009). Moreover, therapy with sFRP2 antibody reduced apoptosis and fibrosis in a hamster model of heart failure (Mastri et al., 2014). These contradictory results of the different studies may be explained by the multiple modes of action of sFRPs (Bovolenta et al., 2008), please refer to section IV.B for details. 
3. Interventions at the Receptor Complex. WNT receptor complexes are formed by members of the FZD protein family in close association with a coreceptor such as LRP5/6 or ROR2. Using a rat model of myocardial infarction induced by permanent coronary artery ligation, our research group was the first to describe the upregulated expression of a member of the WNT/FZD signaling system, $\mathrm{FZD}_{2}$, peaking at 7 days post-MI. The highest $\mathrm{FZD}_{2}$ expression was initially localized in the border zone of the infarct and expanded toward the center of the infarct, together with the infiltration of the area with myofibroblasts (Blankesteijn et al., 1997). The high level of expression of $\mathrm{FZD}_{2}$ and ${ }_{-4}$ in cardiac fibroblasts was confirmed in an unbiased $G$ protein-coupled receptor RT-PCR screen (Snead and Insel, 2012). A similar expression kinetic was observed for the signal transduction molecule DVL1 in a rat MI-model, supporting the concept of activated WNT signaling in the wound healing process (Chen et al., 2004) Later on it was shown that the expression of more members of the FZD family is regulated post-MI: $\mathrm{FZD}_{1},-2,-5$, and -10 were found to be upregulated, whereas $\mathrm{FZD}_{8}$ was downregulated at 7 days post-MI (Barandon et al., 2003). However, to our knowledge, no studies have been published in which the effects of genetic interventions in FZD genes on infarct healing were addressed.

At present, the options for pharmacological intervention in WNT signaling at the level of the FZD receptor are very limited; for details, please refer to section XI. In our group, we investigated the antagonistic effects of peptide fragments of WNT3A and WNT5A on WNT signaling. We identified UM206, a 13-amino acid peptide derived from WNT5A, and tested the effects of subcutaneous infusion of this peptide on infarct healing in a mouse model of permanent coronary occlusion. Administration was performed via osmotic minipump, starting directly after MI induction, and the effect was evaluated at 5 weeks post-MI. UM206 administration reduced LV dilatation, increased cardiac performance, and prevented the development of heart failure. Histologic analysis showed a smaller and thicker infarct and increased myofibroblast numbers in the infarcts (Laeremans et al., 2011). Attenuation of infarct expansion was also observed in UM206-treated swine subjected to cardiac ischemia/reperfusion injury and followed for 5 weeks. In this study, however, the myofibroblast numbers in the infarct area were reduced rather than increased, as was observed in the mice. Moreover, in the UM206-treated group cardiac function was not better than in vehicle-treated controls (Uitterdijk et al., 2016). There are still many open questions regarding the mechanism of action of the peptide inhibitor, because the region from which the peptide is derived does not have a direct interaction with the FZD CRD in the XWNT8/mFZD8 structure published by Janda et al. (2012). However, the attenuation of infarct expansion observed in both studies suggests that the WNT pathway can be a promising target to improve infarct healing a limit post-MI remodeling.

In two recent studies, the effect of the LRP5/6 coreceptors on infarct healing was investigated. As described in detail in section III.C, these coreceptors are specifically involved in WNT/ $\beta$-catenin signaling. In a swine model of ischemia-reperfusion, LRP5 expression was found to be increased only in animals fed a high-cholesterol diet, but not in the group with normal cholesterol diet. Translocation of $\beta$-catenin to the nucleus, as well as increased expression of the WNT target genes Osteopontin and BMP2, confirmed the activation of $\mathrm{WNT} / \beta$-catenin signaling in this group. In the same study, deletion of LRP5 was found to have a deleterious effect on infarct healing in mice. Moreover, human hearts explanted due to ischemic cardiomyopathy expressed higher levels of LRP5 and $\beta$-catenin compared with dilated cardiomyopathy (Borrell-Pages et al., 2016b). These results could be confirmed using mice carrying a cardiomyocyte-specific deletion of LRP5 and 6 (Wo et al., 2016). No studies on the effects of interventions in the coreceptor ROR2 in infarct healing have been published to date.

In several recent studies the role of members of the LRP5/6 inhibitor family DKK in myocardial infarction has been addressed. Plasma levels of DKK1 were found to be higher in patients with ST-segment elevation MI compared with non- ST-segment elevation MI infarcts and can serve as an independent predictor of major adverse cardiovascular events (Wang et al., 2013a). Injection of purified DKK1 protein into the border zone of the infarct area in mice exacerbated the ischemic injury and increased the expression of markers of fibrosis at 4 weeks post-MI. However, cardiac function was not affected by this treatment (Wo et al., 2016). DKK1 and DKK2 were found to have opposite effects on angiogenesis, whereas injection of DKK2 protein in the border zone enhanced the neovascularization of the infarct area with concomitant improved cardiac function (Min et al., 2011). In cultured cardiac fibroblasts, DKK2 reduced proliferation and differentiation into myofibroblasts in a WNT/ $\beta$-catenin dependent manner. This effect could be antagonized by the overexpression of miR-154, which was found to interact directly with DKK2 (Sun et al., 2016). Global deletion of the Dkk3 gene was found to promote apoptosis, inflammation, and remodeling, whereas cardiomyocyte-specific overexpression of DKK $3[\alpha$-myosin heavy chain $(\alpha$-MHC) promoter] had the opposite effect (Bao et al., 2015). In an aptamer-based search for novel candidate biomarkers for cardiovascular disease, DKK4 was found to be upregulated in hypertrophic cardiomyopathy patients at 10 and 60 minutes after a planned septal ablation, a procedure causing cardiac ischemia. The increased circulating DKK4 levels could be confirmed in 
patients suffering from spontaneous myocardial infarction (Ngo et al., 2016). Although these combined studies do not show a uniform role for the different members of the DKK family in infarct healing, they clearly emphasize the importance of $\mathrm{WNT} / \beta$-catenin signaling in multiple processes associated with cardiac remodeling and infarct healing.

4. The $\beta$-Catenin Destruction Complex. Under resting conditions, $\beta$-catenin is continuously degraded by the $\beta$-catenin destruction complex, keeping its intracellular levels low. As described in detail in section V.B, this involves two subsequent phosphorylation steps by CK1 and GSK3, respectively, preparing the protein for ubiquitination and degradation. The role of the two isoforms of GSK3 (GSK3 $\alpha$ and $-\beta$ ) in the remodeling after MI has been investigated using genetic models. Because a full GSK3 $\beta$ knockout is embryonic lethal (Lal et al., 2015), an inducible cardiomyocyte-specific GSK3 $\beta$ deletion was used for these studies (mer-Cre-mer under the control of a $\alpha$-MHC promoter). Despite similar infarct sizes, gene deletion at 5 days post-MI resulted in reduced dilatation of the left ventricle and a better preservation of $\mathrm{LV}$ function in the $\mathrm{KO}$ mice compared with the control group at 8 weeks post-MI. The beneficial effect on cardiac function may be supported by the induction of hypertrophy in the remote myocardium (Woulfe et al., 2010). In contrast to GSK3 $\beta$, global deletion of the GSK3 $\alpha$ gene does not affect the development or the life span of mice (Lal et al., 2015). However, when MI was induced in these mice, increased mortality due to infarct rupture, augmented LV dilatation and attenuated cardiac function were observed. Moreover, the infarct size was increased due to increased apoptosis in the border zone of the infarct (Lal et al., 2012). Remarkably, cardiomyocyte-specific deletion of GSK3 $\alpha$ had the opposite effect on infarct healing, with a better preservation of cardiac function and more viable cardiomyocytes in the border zone. The latter could be attributed to both lower cardiomyocyte apoptosis and higher proliferation rates of the cardiomyocytes (Ahmad et al., 2014). Cardiomyocyte-specific deletion of both GSK3 $\alpha$ and GSK3 $\beta$ (mer-Cre-mer under the control of a $\alpha$-MHC promoter), however, resulted in spontaneous dilatation of the left ventricle and decreased cardiac function (Zhou et al., 2016). Because the two GSK3 homologs are very similar and no subtype-specific drugs are available (Lal et al., 2015), this observation may question the usefulness of GSK3inhibitors in the treatment of acute MI.

To our knowledge, there are no published studies available in which the role of CK1 in infarct healing is addressed by genetic interventions. However, an alternative and translationally interesting approach is the use of the Food and Drug Administration-approved compound pyrvinium. This compound is registered as an anti-helminthic drug, but it has been shown to inhibit WNT signaling by activating $\mathrm{CK} 1 \alpha$ with an
$\mathrm{IC}_{50}$ of around $10 \mathrm{nM}$ (Thorne et al., 2010). A single intramyocardial administration of pyrvinium improved adverse cardiac remodeling and limited the LV dilatation by increasing the proliferation of differentiated cardiomyocytes in the remote myocardium at 30 days post-MI. However, an increased mortality was observed due to toxicity of the intracardiac administration of pyrvinium and no significant improvement of cardiac function was found (Saraswati et al., 2010). In another study, pyrvinium was shown to reduce cardiac fibroblast survival particularly under conditions of low glucose. Oral administration of a pyrvinium pamoate suspension between day 1 and 14 after infarction reduced fibrosis and scar formation in infarcted hearts at 4 and 14 days post-MI, combined with a better ejection fraction than the vehicle-treated mice (Murakoshi et al., 2013). The latter study suggests that oral rather than intracardiac administration would be the preferential route for this drug.

\section{5. $\beta$-Catenin-Mediated Gene Transcription.} Nuclear accumulation of $\beta$-catenin and modulation of the transcription of genes involved in proliferation and survival by this intracellular signaling protein are the final steps in the $\mathrm{WNT} / \beta$-catenin signaling pathway. The availability of $\mathrm{WNT} / \beta$-catenin reporter mice has provided the opportunity to visualize the activation of $\beta$-catenin-mediated WNT signaling in the infarct area. By using a LacZ-reporter mouse driven by the axin2 promotor, the amount of LacZ ${ }^{+}$cells in the border zone was found to increase markedly between day 7 and 21 post-MI. Costainings showed that $\mathrm{LacZ}^{+}$cells coexpressed Sca (hematopoietic stem cells), CD31(ECs), CD45 (leukocytes), von Willebrand factor (ECs), and $\alpha$-smooth muscle actin (myo)fibroblasts), indicating the activation of WNT signaling in multiple cell types (Oerlemans et al., 2010). In a model in which the LacZ expression was driven by a TCF/LEF-responsive promotor (the TOPGAL mouse line), a similar LacZ expression was observed in the border zone and at the epicardial side of the infarct. Many of the $\alpha$-smooth muscle actin-positive cells were found to originate from epicardial ECs that underwent endothelial-tomesenchymal transformation, as shown by cell lineage tracing (Aisagbonhi et al., 2011). In this study, the signal had disappeared at 3 weeks post-MI, which suggests a more rapid completion infarct healing, e.g., due to a smaller infarct size. Recently, the use of an MRI probe consisting of iron-binding human ferritin heavy chain and GFP, under the control of a TCF/LEF sensitive promotor, was described in a rat model of MI. The construct was delivered via an AAV9 vector to the infarcted heart by local injection. Iron accumulation could be detected at the border zone of the infarct of the transduced hearts at 4 weeks post-MI. Histologic analysis confirmed the colocalization of the iron and GFP signals. Inhibition of $\mathrm{WNT} / \beta$-catenin signaling using the compound SEN195 completely abolished the iron 
signal. The combined studies provide evidence for an activation of $\mathrm{WNT} / \beta$-catenin signaling at the borders of the infarct in the first weeks after MI, involving multiple cell types (Matteucci et al., 2016).

Several studies have been executed in which the effect of modulation of $\beta$-catenin levels on infarct healing was studied. Adenoviral gene transfer of a constitutively active form of $\beta$-catenin in cardiomyocytes and cardiac fibroblasts induced proliferation in the latter, whereas hypertrophy and binucleation were observed in the former cell type. In the fibroblasts, an augmented differentiation toward myofibroblasts was observed. Injection of the adenovirus in the border zone of an infarcted rat heart resulted in reduced infarct size due to less apoptosis and cell cycle activation in both cardiac fibroblasts and myocytes (Hahn et al., 2006). The importance of WNT1-mediated activation of WNT/ $\beta$-catenin signaling in the epicardium, promoting epithelial-mesenchymal transition, was also observed using a conditional epicardial $\beta$-catenin knockout model (Duan et al., 2012). However, cardiomyocyte-specific deletion of $\beta$-catenin ( $\alpha \mathrm{MHC}$-Cre PR1 model) has also been reported to have a beneficial effect on survival and left-ventricular function. This was accompanied by a different scar phenotype where cardiac progenitor cells in the subepicardial layer were found to express cardiomyocyte lineage markers (Zelarayan et al., 2008). Furthermore, pharmacological inhibition of the $\beta$-catenin-mediated transcription by administration of the small molecule WNT signaling modulator ICG-001 resulted in improved ejection fraction in female rats at 10 days post-MI (Sasaki et al., 2013). Based on the combined results of these studies, there is no consensus whether $\beta$-catenin signaling should be stimulated or inhibited after MI.

\section{B. WNT Signaling in Valvular Disease}

Heart valves have a typical building plan that is highly conserved in evolution and makes them optimally suited to control the direction of blood flow within the heart. Heart valves consist of three layers: the spongiosa is situated in the middle, flanked by the fibrosa layer on the non-flow side, and the atrialis or ventricularis on the flow side of the valve. The valves are covered by a layer of valvular ECs. The atrialis/ ventricularis is rich in radially oriented elastin fibers, whereas the fibrosa contains high amounts of densely packed collagen fibers that maintain the structural integrity of the closed valve. The spongiosa contains proteoglycans and glycosaminoglycans, allowing some movement of the atrialis/ventricularis and the fibrosa (Orton et al., 2012). The most abundant cell type in the different layers of the valve is the valvular interstitial cell, involved in maintenance of the valve by synthesis and degradation of matrix molecules. Under normal circumstances, valvular interstitial cells resemble quiescent fibroblasts, but upon injury they differentiate into myofibroblasts.

Valvular defects are a common cause of inadequate pump function of the heart. Generally, a distinction is made between valvular stenosis, common in the aortic valve and leading to obstructed outflow, and valvular degeneration, frequently found in the atrioventricular valve and leading to regurgitation of blood from the ventricle into the atrium in the systolic phase. The prevalence of aortic valve stenosis is particularly high in the elderly, with $15 \%-25 \%$ of subjects over 65 year of age being affected (Mathieu et al., 2014). Clinically significant mitral regurgitation due to a myxomatus (degenerated) mitral valve has been reported in $1.7 \%$ of the general population, but this rises to $10 \%$ in the population over 75 year of age (Rajamannan, 2014). The most prominent histologic abnormalities appear to be the formation of bone tissue in the calcified aortic valves (Liu and $\mathrm{Xu}, 2016$ ), whereas cartilage formation can be detected in myxomatous mitral valves (Rajamannan, 2014).

The driving force behind valvular stenosis is calcification of the valve leaflets, the resulting stiffening of the valve leading to an obstruction in the outflow. In many ways this calcification resembles the formation of bone (Rajamannan, 2010; Liu and Xu, 2016). The control of bone formation by WNT/ $\beta$-catenin signaling has been firmly established. Genetic intervention studies have shown that $\beta$-catenin is essential for the differentiation of mature osteoblasts and multiple WNT genes are expressed in either osteoblast precursors or adjacent cells during embryonic development (Hartmann, 2006). Moreover, mutations in LRP5 are associated with either high bone density (Boyden et al., 2002) or a genetic form of bone loss called osteoporosis pseudoglyoma syndrome (Gong et al., 2001), underscoring the central role of $\mathrm{WNT} / \beta$-catenin signaling in the regulation of bone density. These observations have induced extensive studies of involvement of WNT signaling in valvular defects. The expression of osteoblastic genes is controlled by the balance between Notch and WNT/ $\beta$-catenin signaling (Mathieu et al., 2014), and indeed elevated levels of LRP5 were reported in calcified aortic valves and, to a lesser extent, degenerative mitral valves (Caira et al., 2006). Moreover, a positive association between circulating DKK1 levels and calcified aortic stenosis was recently reported in patient with angiographically normal coronary arteries, but this association was less clear in the presence of concomitant atherosclerosis of the coronaries (Motovska et al., 2015).

Involvement of $\mathrm{WNT} / \beta$-catenin signaling is not restricted to valvular calcification but was recently implicated in myxomatous valve disease as well. Myxomatous valve diseases is characterized by a thickening of the valvular leaflets and alterations in the extracellular matrix. It is the result of altered growth factor signaling, among which Wnt, BMP, and Notch 
are important participants. Excessive deposition of proteoglycans and a concomitant degradation of collagen and elastin are characteristics of myxomatous valve disease, where the phenotype of the spongiosa resembles that of cartilage (Orton et al., 2012). Loss of $\beta$-catenin signaling in valvular interstitial cells activated the transcription factor Sox9 and subsequent expression of chondrogenic matrix genes (Fang et al., 2014). Inactivation of axin2, a negative regulator of $\mathrm{WNT} / \beta$-catenin signaling, showed immature heart valves and a progressive myxomatous valve degeneration in mice at 4 months of age, underscoring the importance of this signaling pathway in valve homeostasis (Hulin et al., 2017).

\section{WNT Signaling in Cardiac Arrhythmias}

Cardiomyocytes are attached to each other by specialized structures called intercalated disks. These intercalated disks not only provide strong mechanical connections between cardiomyocytes but also allow the fast propagation of electrical signals through the myocardial tissue, which is an essential feature for their simultaneous and coordinated contraction. Three main structures can be identified in the intercalated disk: desmosomes, anchoring the adjacent cells; adherens junctions, connecting actin filaments to the cell adhesion complex; and gap junctions, involved in the electric and metabolic coupling of neighboring cells. Although these structures are often studied separately, it is becoming increasingly clear that all intercalated disk components closely cooperate and should be seen as an integrated functional unit of the cardiomyocyte (Vermij et al., 2017). Therefore the term area composita was introduced to emphasize the integrated nature of desmosomes and adherens junctions (Patel and Green, 2014).

Apart from their role in WNT signaling, members of the catenin family are an important component of both desmosomes and adherens junctions. In the latter, $\mathrm{N}$-cadherins are single membrane-spanning proteins which form intercellular connections via interaction of the extracellular domains extending from two neighboring cells. On the cytoplasmic side, N-cadherin is attached to the actin cytoskeleton via a complex consisting of $\alpha$ - and $\beta$-catenin and plakoglobin, also known as $\gamma$-catenin. Desmosomes are sturdy, symmetrical anchoring structures that provide structural support to the cell-cell junction. They are not involved in the connection with the actin cytoskeleton but they connect to intermediate filaments. Desmosomes consist of the single membrane spanning cadherins desmoglein and desmocollin. Desmin is bound to these cadherins via a complex formed by plakoglobin, plakophilin, and desmoplakin. Gap junctions consist of complexes of gap junction proteins from the connexin $(\mathrm{Cx})$ family, forming a tubular structure. The typical ventricular gap junction consists of a complex of six $\mathrm{Cx} 43$ proteins in the plasma membrane, connected to a similar complex in the neighboring cell. These structures allow the rapid propagation of action potentials and transport of small molecules between cells (Patel and Green, 2014). Other members of the connexin family, including $\mathrm{Cx} 40$ and $\mathrm{Cx} 45$, have been identified in fast-conducting structures such as the His/Purkinje system (Lo, 2000).

There are several lines of evidence that the expression of $\mathrm{Cx} 43$ is under the control of WNT signaling. First, activation of WNT/ $\beta$-catenin signaling in neonatal rat cardiomyocytes by coculturing with WNT1secreting cells or with the GSK3-inhibitor lithium chloride ( $\mathrm{LiCl})$ resulted in an increase in $\mathrm{Cx} 43$ expression (Ai et al., 2000). Rapid electrical stimulation of neonatal rat cardiomyocytes resulted in nuclear translocation of $\beta$-catenin within 10 minutes and an increase in $\mathrm{Cx} 43$ expression within 60 minutes, suggesting a $\mathrm{WNT} / \beta$-catenin-mediated regulation. This was accompanied by a significant increase in conduction velocity in these cells. Moreover, in a cardiomyopathic mouse model showing arrhythmias and remodeling of gap junctions, decreased levels of $\beta$-catenin and $\mathrm{Cx} 43$ were observed (Nakashima et al., 2014). Recently, mutations in the lamin $\mathrm{A} / \mathrm{C}$ gene ( $L M N A$ ) causing cardiomyopathy with conduction abnormalities were shown to decrease $\mathrm{WNT} / \beta$-catenin signaling, which was accompanied by an increase in sFRP expression and reduced expression of $\mathrm{Cx} 43$ (Le Dour et al., 2017).

The involvement of aberrant WNT signaling in arrhythmogenic cardiomyopathy (AC) has been extensively studied and documented. AC is an inheritable cardiac disease characterized by life-threatening ventricular arrhythmias and heart failure and is a major cause of death in young athletes. Originally the condition was thought to be concentrated in the right ventricle, giving rise to the name arrhythmogenic right ventricular cardiomyopathy, which is now replaced by AC. A typical pathologic finding in these hearts is the replacement of cardiomyocytes by adipose and fibrous tissue (Pilichou et al., 2016). In most families, AC is inherited in a autosomal dominant fashion with incomplete penetrance. Mutations in five genes encoding the desmosomal proteins plakoglobin, desmoplakin, plakophilin-2, desmoglein-2, and desmocollin-2 have been reported in about half of the affected patients, therefore AC is generally considered a desmosomal disease (Sen-Chowdhry et al., 2005; Basso et al., 2011). The link between AC and WNT/ $\beta$-catenin signaling can be found in desmosomal crosstalk with the nucleus. Decreasing desmoplakin expression via siRNA in cultured atrial myocytes resulted in nuclear translocation of plakoglobin, which competes with $\beta$-catenin for interaction with the TCF/LEF transcription factors. This induced a twofold reduction in $\mathrm{WNT} / \beta$-catenin signaling and induced the expression of adipogenic and fibrogenic genes as observed in AC patients. An AC phenotype was also found in mice by the heterozygous 
deletion of the desmoplakin ( $D s p$ ) gene (Garcia-Gras et al., 2006) and in mice with a cardiomyocyte-specific silencing of $\beta$-catenin and plakoglobin (MerCreMer under control of the $\alpha \mathrm{MHC}$ promoter) (Swope et al., 2012). Genetic fate mapping experiments have shown that the adipocytes in AC hearts originate from second heart field progenitor cells, in which the attenuated $\mathrm{WNT} / \beta$-catenin signaling resulting from nuclear plakoglobin induced an adipogenic fate (Lombardi et al., 2009 ). The importance of $\mathrm{WNT} / \beta$-catenin signaling in AC was recently illustrated in two mouse models with mutated desmosomal proteins (Chelko et al., 2016). The mice showed GSK3 accumulation in the intercalated disks, similar to those observed in AC patients. Treatment with SB216763, a GSK3 inhibitor, normalized the protein distribution in the intercalated disks and improved ventricular fibrosis and inflammation, pointing to GSK3 as a therapeutic target in AC.

\section{WNT Signaling in Cardiac Hypertrophy}

Cardiac hypertrophy is an adaptive response of the heart to a wide range of external factors and neurohormonal/mechanical stimuli, such as pressure overload, $\beta$-adrenergic stimulation, MI, etc. These factors activate different signaling pathways and key molecules, including, but not restricted to, NFAT, PI3K/ Akt, GATA4, CaMKII, PKC, ERK, JNK, calcineurin (Hunter and Chien, 1999), and WNT (Blankesteijn et al., 2008). Hypertrophy can have profound effects on pathologic cardiac remodeling, which progresses to impairment of cardiac function and eventually leads, when left untreated, to lethal congestive heart failure. In various studies, cardiac hypertrophy has been linked with changes in WNT signaling and here we will discuss the involvement of various WNT components in the mediation of pro- and antihypertrophic stimuli.

1. Involvement of WNT, FZD, and LRP5 / 6 and DKK. The data on WNT ligand involvement in the development and progression of myocardial hypertrophy is quite scarce. He et al. (2015) used an isoprenalineinduced hypertrophy mouse model and reported that both $\beta$-catenin-dependent and -independent WNT signaling cascades are activated in hypertrophic hearts, as was suggested by increased WNT3A and WNT5A expression, respectively. Both $\beta$-catenin and non- $\beta$-catenin signaling cascades were confirmed to play an essential role in cardiomyocyte hypertrophy as shown by other groups. Exogenous stimulation of primary cardiomyocytes with WNT5A produces a pronounced increase in cardiomyocyte growth and protein synthesis, whereas the WNT component Dapper-1 was found to play a crucial role in this effect (Hagenmueller et al., 2014). Interestingly, the same group has also shown that WNT3A mediates its prohypertrophic effects on cardiomyocytes via Dapper-1, showing that the $\beta$-catenin-dependent pathway (via DVL2) is heavily involved (Hagenmueller et al., 2013). On the other hand, Jin et al. (2015b) proposed a beneficial effect of WNT5A on hypertrophy of the right ventricle (RV). Using a hypoxia-induced pulmonary hypertension mouse model, they established that exogenous treatment with recombinant mouse WNT5A suppresses cardiomyocyte growth via inhibition of the $\beta$-catenin/cyclin D1 axis.

Our group used the method of subtractive hybridization and showed more than 20 years ago that $\mathrm{FZD}_{2}$ is immediately upregulated following aortic banding in rats, whereas levels remain elevated for at least 10 days after surgery (Blankesteijn et al., 1996). The involvement of $\mathrm{FZD}_{2}$ in cardiac hypertrophy was also observed by Cerutti et al. (2006), who performed microarray studies and showed a positive correlation between $\mathrm{FZD}_{2}$ expression and $\mathrm{LV}$ mass index in different hypertensive/hypertrophic animal models [spontaneously hypertensive rat, Lyon hypertensive rat, and heterozygous TGR9(mRen2)27 rat].

Alapati et al. (2013) made a link between LRP5/6 and RV hypertrophy, which is exacerbated in a hyperoxiainduced neonatal lung injury mouse model. Hyperoxiainduced neonatal lung injury has pronounced effects on the lungs, leading to pulmonary hypertension and RV hypertrophy, and this is linked to activation of WNT/ $\beta$-catenin signaling. By making use of a WNT-LRP5/6 complex blocker, called Mesd, they show that the activation of WNT signaling is suppressed and both pulmonary hypertension and RV hypertrophy are attenuated. Thus blockade of LRP5/6 can produce antihypertrophic effects on the RV.

Phillips et al. (2011) investigated the effects of a double KO of $D k k 1$ and $D k k 2$ in mice during early development. The double $D k k 1 / 2 \mathrm{KO}$ mouse genotype is perinatally lethal; however, it is interesting that embryonic hearts are thickened at embryonic day (E) 10.5. At E12.5 it is found that the double null mice demonstrate increased numbers of cardiomyocytes (thus myocardial hyperplasia), an effect that is long lasting. On the other hand, the epicardium is also affected by the altered WNT signaling activity; however, any epicardial changes observed early are resolved later on (by E15.5).

More attention has been devoted to the DKK3 isoform. Cerutti et al. (2006) were probably the first to demonstrate a negative correlation between LV mass augmentation and DKK3 in hypertensive rats. The study of Zhang et al. (2014) suggested that DKK3 is heavily involved in the regulation of pathologic hypertrophy: Patients suffering from dilated cardiomyopathy (DCM), as well as mice that are subjected to 4 weeks of aortic banding show robustly downregulated DKK3 expression levels, whereas $\beta$-myosin heavy chain (MHC) and atrial natriuretic factor, both markers of hypertrophy, are decreased in both cases. Additionally, DKK3 expression is suppressed in cultured neonatal cardiomyocytes following AngII or phenylephrine stimulation. When DKK3 is knocked down in these cells, 
cardiomyocyte hypertrophy worsens but when DKK3 expression is induced this effect is reversed. Lastly, mice overexpressing DKK3 were subjected to aortic banding. The authors report that hypertrophy is drastically attenuated 8 weeks after surgery in these mice compared with their WT counterparts, clearly demonstrating the cardioprotective effects of DKK3 (Zhang et al., 2014). In addition, DKK3 is also a crucial player in the development of familial dilated cardiomyopathy ( $\mathrm{Lu}$ et al., 2016). Levels of DKK3 expression are paradoxically found to be elevated in two mouse models of DCM (cTnT $^{\mathrm{R} 141 \mathrm{~W}}$ and adriamycin-induced DCM), opposite to the findings of Zhang et al. (2014). Lu et al. (2016) show that deletion of DKK3 exacerbates cardiac remodeling; however, when DKK3 is transgenically overexpressed, it upregulates the $\beta$-catenin-dependent signaling (via observed effects on DVL1, $\beta$-catenin, c-myc, and AXIN2) and downregulates the $\beta$-catenin-independent signaling (via suppression of JNK, CAMKII, and HDAC4). DKK3 appears to be a cardioprotective factor in this case; however, it is an enigma how it can induce rather than downregulate the WNT cascade to mediate this effect.

2. The Role of Secreted Frizzled-Related Proteins in Cardiac Hypertrophy. Most of the studies implicating sFRP in mediating cardiac hypertrophy are quite recent. Sklepkiewicz et al. (2015) demonstrated that an active sFRP1 expression is crucial for LV remodeling and hypertrophy, and is especially relevant in the aging organism. Sfrp1 KO mice exhibit robust hypertrophy already at 6 months of age compared with their WT counterparts, as shown by increased HW/BW ratio and LV wall thickness. This difference was even more pronounced at 12 months of age, and this coincided with upregulated WNT1/-3/-7b mRNA expression, induced $\beta$-catenin protein levels, and LEF1 expression in the KOs. Furthermore, hypertrophy in the Sfrp1 KO was accompanied by profound changes in LV diastolic and systolic function, as well as presence of $\beta$-catenin in the intercalated disks, possibly contributing to myocardial stiffening (Sklepkiewicz et al., 2015).

Downregulation of plasma membrane calcium ATPase isoform 4 (PMCA4), either by genetic intervention or by pharmacological antagonism, revealed that sFRP2 expression is induced in fibroblasts. In addition, the mice lacking PMCA4 (and thus overexpressing sFRP2, a protein exerting antihypertrophic activity) demonstrated a high survival 3 months after aortic banding. sFRP2 secreted by fibroblasts was shown to be the crucial regulator (Mohamed et al., 2016).

Askevold et al. (2014) suggested that sFRP3 could inhibit cardiac hypertrophy by targeting WNT signaling. HF patients demonstrated elevated sFRP3 levels compared with control patients, a finding that was correlated with increased mortality. Indeed, sFRP3 and -4 are shown to be upregulated in failing human hearts, leading to an attenuation of WNT signaling
(Schumann et al., 2000). Nevertheless, keeping in mind conflicting reports on the effect of sFRP3 on WNT signaling (Zi et al., 2005; Galli et al., 2006), further studies are warranted to ascertain the potential of sFRP3 as an antihypertrophic therapy tool.

Exposure of cardiomyocytes to AngII is reported to induce sFRP5 expression, along with $\mathrm{BNP}$ and $\mathrm{TNF} \alpha$, which are indicators of hypertrophic growth. Interestingly, when cardiomyocytes were preincubated with sFRP5 before the AngII challenge, sFRP5 could salvage hypertrophy, as shown by suppressed BNP and TNF $\alpha$ protein levels (Jin et al., 2015a).

3. The $\beta$-Catenin Destruction Complex. The destruction complex is the limiting factor of $\beta$-catenin-mediated WNT signaling, regulating the free $\beta$-catenin levels and preventing its stabilization and nuclear translocation. Various studies have demonstrated an involvement of the destruction complex components in the development of cardiac hypertrophy (Kerkela et al., 2007). Adult hypertrophic hearts show suppressed levels of APC mRNA expression compared with their healthy counterparts. This finding is in accordance with induced $\beta$-catenin protein levels in the hypertrophic hearts, signifying an activation of the WNT cascade (Rezvani and Liew, 2000).

Our group has provided compelling evidence for the essential role of DVL1 in the regulation of cardiac hypertrophy. Dvl1 KO mice were subjected to aortic banding and were followed for 7, 14, and 35 days. Aortic banding induces hypertrophy, as shown by increased HW/tibia length ratios, in WT mice 7, 14, and 35 days post-op, but this is evident after only 35 days in the Dvl1 KO mice, implying that deletion of the $D v l 1$ gene delays induction of hypertrophy. In the same way, posterior wall thickness was increased in WT mice but only mildly and later in the KOs. In addition, 7 days after operation, the expression of both ANF and BNP (markers of hypertrophy development) was more than doubled in the WT compared with their KO counterparts. These results suggest a detrimental effect of DVL1 following aortic banding. GSK3 $\beta$ activity was found to be upregulated in the Dvl1 KO mice, which was reflected in lower $\beta$-catenin mRNA and protein expression. In contrast, Dvl1 WT mice showed an increase in $\beta$-catenin expression (as well as an upregulation of p-Akt, which is known to induce hypertrophy), implying an activation of the $\mathrm{WNT} / \beta$-catenin cascade under normal conditions (van de Schans et al., 2007). In agreement with the aforementioned findings, Malekar et al. (2010) showed that overexpression of DVL1 can have deleterious effects on the development of hypertrophy and cardiac remodeling in mice, 4 weeks after aortic banding. Cardiomyocyte size in Dvl1 overexpressing mice was increased by a staggering $86 \%$ and the HW/BW ratio was significantly increased compared with WT; this leads to LV dilatation, an almost halving in $\mathrm{EF}$ and an impairment in contractility. Both 
$\beta$-catenin-dependent (via $\beta$-catenin, cyclin D1, c-myc, and axin2) and -independent (via JNK, CAMKII, and PKC) signaling routes were found to be activated in the DVL1 overexpressing mice, contributing, probably synergistically, to a severe cardiomyopathy phenotype.

When the Dvl1 overexpressing mice were crossed with CAMKIII $\gamma$ KO mice, the hypertrophy and cardiomyopathy phenotype was lost; it is interesting to note that the authors could not demonstrate any difference in $\mathrm{PKC}, \mathrm{JNK}$, or $\beta$-catenin activation following the deletion of CAMKIIS / $\gamma$ in the Dvl1-overexpressing mice (Zhang et al., 2015c).The transgenic deletion of CaMKIIS $\gamma$ halts hypertrophy of Dvl1-overexpressing mice, as verified by substantial increases in heart size, myocyte area, HW/tibia length, fibrosis, and hypertrophy marker gene expression (ANF and $\beta$-MHC). The findings demonstrated clearly that all branches of WNT signaling are taking part in the mediation of cardiac hypertrophy.

The group of Hardt investigated the involvement of DVL2 in cardiac hypertrophy (Hagenmueller et al., 2013). The authors indicate Dapper-1 as a key regulator in DVL2-mediated hypertrophy and LV remodeling. Mice overexpressing Dapper1 showed elevated HW/BW and exacerbated cross-sectional area of the myocytes, as well as induction of hypertrophy markers (ANF, BNP, and $\beta$-myosin). These mice exhibited high DVL2 and $\beta$-catenin expression. In the meantime, in vitro work showed that Dapper-1 is an essential coregulator of the $\beta$-catenin-dependent WNT-mediated hypertrophy in cardiomyocytes. Dapper-1 downregulation leads to an attenuation of WNT signaling cascade ( $\beta$-catenin and c-myc protein levels), whereas its overexpression increases both $\beta$-catenin and TCF/LEF activity.

GSK3 is a ubiquitous Ser/Thr kinase that plays an essential role in a multitude of cellular functions (Kerkela et al., 2007). GSK3 phosphorylates a wide range of substrates and suppresses their activities, mediating decisively both $\beta$-catenin-dependent and -independent cascades. GSK3 $\beta$ has been shown to play an essential role in cardiac hypertrophy (Hardt and Sadoshima, 2002). It should be noted that the other isoform of GSK3, GSK3 $\alpha$, is also known to play crucial roles in cardiac hypertrophy and appears to have a rather antihypertrophic effect mediated mainly via ERK (Zhai et al., 2007) and E2F (Matsuda et al., 2008). Although several reports demonstrate that GSK3 $\alpha$ might actually be even more important in aortic banding-induced hypertrophy than GSK3 $\beta$ (Cheng et al., 2011a), it is the latter that mediates its effects also via the WNT signaling pathway. However, an extensive discussion of GSK3 $\alpha$ is beyond the scope of the current review.

Interestingly, it is not exclusively the $\mathrm{WNT} / \beta$-catenin signaling component of GSK3 $\beta$ that is involved in this prohypertrophic mediation (Hirotani et al., 2007); GATA4 (Liang and Molkentin, 2002), NFAT (Antos et al., 2002), CREB, PI3K/Akt, PKA, and ERK (El Jamali et al., 2004), eIF2 (Hirotani et al., 2007), JAK/ STAT (Clerk et al., 2007), and others play equally essential roles. However, as discussed earlier, the activity of GSK3 $\beta$ is regulated by its phosphorylation status: phosphorylation at the $\mathrm{Ser}^{9}$ residue leads to inhibition of GSK3 $\beta$ (Sutherland et al., 1993), resulting in activation of WNT signaling, whereas phosphorylation at the $\mathrm{Thr}^{216}$ residue is inducing the activity of the kinase (Liang and Chuang, 2007).

Haq et al. (2003) reported that either hypertrophic stimulation (by phenylephrine and endothelin-1) of neonatal rat ventricular myocytes, or aortic banding in rats, activates PKB to phosphorylate GSK3 $\beta$ at Ser 9 . This phosphorylation induces inactivation of GSK $3 \beta$, stabilization of $\beta$-catenin, and as a consequence an increase in myocyte size and protein synthesis. A similar effect was demonstrated by Morisco et al. (2000) following an isoprenaline challenge of myocytes, leading to the hypothesis that the $\beta$-adrenergic system mediates its prohypertrophic effects via the Akt/GSK3 $\beta$ signaling.

A rather contradictory finding by Haq et al. (2001) was that GSK3 $\beta$ signaling is suppressed in failing hearts (end-stage HF) but not in hypertrophic ones, implying that GSK3 $\beta$ upregulation in overload in humans might be short-lived $(<4$ weeks?). Tateishi et al. (2010) studied the effect LiCl-mediated GSK3 $\beta$ inhibition in a model of pressure overload-induced cardiac hypertrophy in the rat. $\mathrm{LiCl}$ treatment resulted in higher LV weight, increased posterior wall thickness, and increased mRNA expression of the prohypertrophic marker atrial natriuretic factor compared with untreated rats exposed to aortic banding. Thus inhibition of GSK3 $\beta$ is found to exacerbate hypertrophy. Data from $G s k 3 \beta$ null mice seem to support these observations. Deletion of Gsk3 $\beta$ in mice led to severe hypertrophic cardiomyopathy and cardiac-related death of mice pups due to a wide range of cardiac defects (Kerkela et al., 2008). These mice exhibited substantially induced proliferation of cardiomyocytes, leading to increased LV/RV free wall and IVS thickness. The molecular basis of these findings was proposed to be an increased expression and nuclear localization of GATA4, cyclin $\mathrm{D} 1$, and c-Myc, all three regulators of cell proliferation. Hirotani et al. (2007) generated Tg mice with an $\alpha \mathrm{MHC}$ driven cardiac-specific expression of dominant negative GSK3 $\beta$ (Tg-GSK3 $\beta$-DN). These mice exhibit physiologic (adapted) hypertrophy after $2.5,4,6$, or 8 months, while also showing increased contractility compared with WT counterparts. When they are subjected to aortic banding, the GSK3 $\beta$ inhibition does not appear to produce any further effects implying that GSK $3 \beta$-DN is mainly mediating basal state hypertrophy and not aortic banding-related hypertrophy (but still, decreased GSK3 $\beta$ activity appears to protect the heart from decompensated hypertrophy). On the other hand when 
GSK3 $\beta$ was transgenically overexpressed (conditional induction regulated by doxycycline), mice developed LV dysfunction and augmented heart weight/tibia length ratios, as well as increased apoptosis, stressing that decreased GSK3 $\beta$ activity in the diseased heart is cardioprotective. Interestingly, a GSK3 inhibitor called 6-bromoindirubin-3'-oxime (BIO) induced neonatal and adult cardiomyocyte proliferation, despite the fact that the myocyte is regarded in general as a terminally differentiated cell (Tseng et al., 2006). It seems that the activation of the WNT cascade might be stimulating dedifferentiation and proliferation of mammalian myocytes, indicating another mechanism for GSK3's effects on hypertrophy, without though excluding the possibility of BIO having off-target activity, because it is not selective for GSK3 isoforms (Cheng et al., 2011a).

Ser $^{9}$ replacement by Ala (which renders GSK3 $\beta$ insensitive to phosphorylation and thus inhibition) suppresses cardiac hypertrophy induced by overexpression of calcineurin, 9 days of $\beta$-adrenergic stimulation with isoproterenol, or 3 weeks of aortic banding (Antos et al., 2002), as shown by increased heart weights and upregulated expression of markers of hypertrophy (BNP, ANF, $\beta$-MHC). Using a doxicyclin-inducible overexpression of the same GSK3 $\beta$-mutant, Sanbe et al. (2003) observed a similar suppression of the hypertrophic response to pressure overload. Expression of inactivation-resistant GSK $3 \alpha / \beta$ isoform was shown to be cardioprotective following beta-adrenergic challenge with isoprenaline (Webb et al., 2010). Normal heart weight as well as LV thickness were preserved in these mice, underscoring the importance of a normally functioning GSK $3 \beta$ system. On the other hand, abnormal upregulation of GSK3 $\beta$ can be deleterious for the myocardium. GSK3 $\beta$ features as a crucial negative regulator of hypertrophic growth of the LV. As reported by Michael et al. (2004), transgenic mice with an approximately ninefold overexpression of wild-type GSK3 $\beta$ showed dramatically reduced growth with structural and functional abnormalities in both ventricles at 2 and 5 months of age (including reduced wall thickness and internal long-axis dimension of the LV). An interesting observation was that the differences in heart size of WT and GSK3 $\beta$-Tg were much smaller, which may suggest that the differences observed later in life are due to disruption of hypertrophic growth (mediated obviously by GSK3 $\beta$ ) rather than from proliferation of cardiomyocytes, which primarily takes place during embryonic development.

The results described above suggest that the blockade and the overexpression of GSK3 $\beta$ are equally noxious to the heart under conditions inducing hypertrophy. Thus, a precise balance of GSK $3 \beta$ is of paramount importance. What is characteristic about GSK3 $\beta$ is that there is quite some interest for the identification of potent and selective GSK3 $\beta$ activators and inhibitors, to suppress or activate, respectively, WNT signaling. High- throughput screening methods are under way and there is plenty of potential for novel compounds that will be able to adequately stimulate or block (depending on the context) this kinase (Cohen and Goedert, 2004; An et al., 2010; Takahashi-Yanaga, 2013). On the other hand, it is paradoxical that this interest on the bench level does not materialize in clinical trials investigating GSK3 $\beta$ modifying agents that could be used as potentially novel tools against cardiac hypertrophy (Cheng et al., 2011a). A search in clinicaltrials.org showed that the only agents affecting GSK3 $\beta$ under investigation are the "old school" valproate, $\mathrm{LiCl}$, and the novel NP031112 (GSK3 inhibitor), however, none is under evaluation in a cardiovascular study.

4. $\beta$-Catenin-Mediated Gene Transcription. As expected by its key role in the regulation of WNT signaling, there is a myriad of studies that have focused on $\beta$-catenin in an effort to shed light on the mechanisms regulating cardiac hypertrophy. It is well established that the multiple functions exhibited by $\beta$-catenin are determined by its phosphorylation and stabilization status: the spatial localization of $\beta$-catenin is heavily dependent on its $\mathrm{Tyr}^{142}$ and $\mathrm{Tyr}^{654}$ phosphorylation (Harris and Peifer, 2005). The study of null mutations of $\beta$-catenin is precluded by the fact that such mutations are embryonically lethal (Haegel et al., 1995). Actually, both gain and loss of function of $\beta$-catenin lead to severe developmental defects (Grigoryan et al., 2008). Thus, the design of conditional mutants is the only alternative to investigate the role of $\beta$-catenin in (patho)physiology in the mouse. Two approaches are available: 1) one producing tissuespecific null mutation and so, a conditional loss-offunction of WNT signaling and 2) another one producing a constitutively stabilized $\beta$-catenin by deleting exon 3 of $\beta$-catenin gene, leading to a gain-of-function of WNT signaling (Grigoryan et al., 2008).

In a hallmark paper, Haq et al. (2003) showed not only that the WNT/ $\beta$-catenin signaling is important for the growth of cardiomyocytes in the context of hypertrophy, but also that $\beta$-catenin activation is necessary, although not necessarily independent because PKB also seems to be profoundly involved, for the induction of hypertrophy in vitro. $\beta$-Catenin levels are upregulated in cardiomyocytes that are challenged with various hypertrophic stimuli (phenylephrine, endothelin-1, or aortic banding), whereas $\beta$-catenin stabilization can induce protein synthesis and cardiomyocyte enlargement, but not ANF expression, sarcomere organization, or cardiomyocyte proliferation (Haq et al., 2003). Still, it appears rather unlikely that these effects are WNT-mediated. Overexpression of $\beta$-catenin via adenoviral gene transfer also robustly affects cellular size, protein synthesis and nuclear polyploidization, all indications of hypertrophy of cardiomyocytes, without having any effects on proliferation of cardiomyocytes, while apoptosis of cardiomyocytes was also reported as a beneficial effect of 
$\beta$-catenin upregulation (Hahn et al., 2006). Similar effects were observed on cardiac fibroblasts, albeit less pronounced. Conversely, gene transfer of $\beta$-catenin mutants in which transcriptional activity is disrupted $(\mathrm{NCad} \Delta \mathrm{C})$, reversed the aforementioned effects on both myocytes and cardiac fibroblasts. Zhang et al. (2009a) used siRNA to knockdown $\beta$-catenin in cardiomyocytes. They observed a suppression of phenylephrine-induced protein synthesis, a reduction of the transcription levels of the hypertrophic marker ANF, and a significantly depressed cardiomyocyte cell area. The study also demonstrated that the ANF promoter contains a LEF1 binding site, to which $\beta$-catenin is recruited during phenylephrine stimulation.

The $\beta$-catenin/TCF/LEF axis was shown to be an essential component of hypertrophic growth in myocytes by Chen et al. (2006). Mice carrying a myocytespecific deletion of $\beta$-catenin (Lef-120) exhibit an attenuated hypertrophic response (as exhibited by dramatically reduced myocyte growth) and substantially improved cardiac function after aortic banding compared with mice with preserved $\beta$-catenin expression. Cardiac-specific deletion of $\beta$-catenin (via Cre recombinase under the control of a $\alpha \mathrm{MHC}$ promoter) shows a demised TAC-induced hypertrophy 4 weeks after banding (Qu et al., 2007). Prohypertrophic gene expression was dramatically altered in these mice, with enhancement of fetal gene expression (ANF, BNP, $\beta$-MHX, SERCA, etc.) being evident. Baurand et al. (2007), on the other hand, challenged the concept of low $\beta$-catenin expression being beneficial by showing an exacerbation of spontaneous cardiac hypertrophy in cardiomyocyte-specific $\beta$-catenin-depleted mice $(\alpha$ MHC-Cre-PR1 model); mice exhibiting stabilized $\beta$-catenin were reported to show attenuation of AngII-induced cardiac hypertrophy, as shown by cardiomyocyte size and ANF expression. Interestingly, the reduced hypertrophy in the latter mice came to the expense of declined heart function (as indicated by reduced ejection fraction). These contradicting results could possibly be explained by the fact that different models (aortic banding vs. AngII stimulation) activate different signaling pathways, resulting in different responses on the cardiomyocytes.

A final point of interest is the exact mechanism by which $\beta$-catenin modulates the hypertrophic state of cardiomyocytes. As discussed before, $\beta$-catenin resides at the adherens junctions in the catenin/cadherin complex and plays important roles in cell adhesion and cell-cell communication (Harris and Peifer, 2005). $\mathrm{mXin} \alpha$, a protein of the intercalated disk (an important point where sarcomeric organization takes place during cardiac hypertrophy), was found to contain a $\beta$-cateninbinding domain, implying that $\beta$-catenin can play a key role in bundling of actin filaments (Choi et al., 2007). Interestingly, $\beta$-catenin is found to accumulate in the intercalated disks of myocytes in hypertrophic cardiomyopathy hamsters, possibly leading to induced wall stiffness of the myocardium and deleterious structural and functional effects associated with hypertrophic cardiomyopathy (Masuelli et al., 2003). Zheng et al. (2013) reported the exact opposite observation, showing that $\beta$-catenin presence is suppressed in the intercalated disks and increased in the myocyte nucleus of spontaneously hypertensive heart failure (SHHF) rats (Zheng et al., 2013). This finding might at first look paradoxical; however, it could be explained by the variability of the two models (as the SHHF is more of a heart failure model, whereas the hypertrophic cardiomyopathy is rather hypertrophic model without progression to heart failure).

In conclusion, stabilized $\beta$-catenin is sufficient (and as shown, necessary) for the induction of hypertrophic growth in cardiomyocytes. Nevertheless, the precise roles of $\beta$-catenin are still not fully understood with variable reports from in vitro and constitutive mutation studies. More in-depth investigations are required to clarify the exact mechanisms by which $\beta$-catenin (and WNT signaling in general) exhibits its sophisticated effects on the hypertrophic response. This will provide new options for interfering with the signaling to halt cardiac hypertrophy and prevent its adverse effects.

\section{E. WNT Signaling and Heart Failure}

Heart failure (HF) is a progressively disabling chronic disease, which comes as an aftermath of a variety of pathologies, most importantly MI and hypertrophy (covered above). Initially, following injury, endogenous compensatory mechanisms prevent cardiac failure; however, eventually, cardiac remodeling produces a detrimental effect on cardiac architecture and function and leads to lethal HF. HF is a major public health issue, and the total number of HF patients around the globe is estimated to be more than 26 million (Ambrosy et al., 2014). According to the latest statistics from the American Heart Association (Benjamin et al., 2017), about $20 \%$ of people suffering an MI after 65 years of age will develop HF, and approximately one in two patients diagnosed with HF will die within 5 years. The prognosis for the United States alone is even worse because the $\mathrm{HF}$ prevalence is expected to rise $46 \%$ by 2030 . It is obvious that the financial impact of HF is enormous: in 2012 the total cost associated with HF in the United States was more than $\$ 30$ billion, and the estimations are expected to increase to $\$ 69$ billion by 2030 . Currently, available anti-HF pharmacotherapy is not curative but mainly directed at the symptoms and it only attenuates the deterioration to end-stage HF, without completely preventing it. WNT signaling has been shown to be involved in mediating adverse cardiac remodeling and the progression to $\mathrm{HF}$, thus interventions in the pathway may provide new targets for HF therapy. 
1. Extracellular Signaling-Secreted FrizzledRelated Proteins. Inactivation of the Sfrp1 gene has been reported to cause hypertrophy, fibrosis, and depressed cardiac function in old (6 and 12 month old) mice. In these mice, $\beta$-catenin, WISP-1, and LEF1 levels are induced. In agreement with these findings, the authors show that sFRP1 is downregulated and WISP-1 is induced in human dilated cardiomyopathy and ischemic cardiomyopathy (Sklepkiewicz et al., 2015). As mentioned before, transgenically overexpressed FrzA (sFRP1) exhibits a cardioprotective effect following MI. Infarct scar is strengthened, myofibroblast presence is augmented, whereas more and thicker blood vessels/capillaries lead to better perfusion of the ischemic tissue. These observations, in combination to the improved cardiac function as attested by echography, suggest a cardioprotective profile of sFRP1 overexpression, which could be exploited to prevent $\mathrm{HF}$ progression (Barandon et al., 2003). In the same context, sFRP1 levels were shown to be downregulated in patients suffering from $\mathrm{HF}$ but this effect was reversed following left ventricular assist device (LVAD) support (Felkin et al., 2011). The role of sFRP2 in heart failure development is less clear: On the one hand, injection of sFRP2 was shown to have beneficial effects on cardiac function in a hamster model of congestive heart failure with reduced myocyte apoptosis and fibrosis (Mastri et al., 2014), whereas injection of sFRP2 into the rat infarct leads to suppression of fibrosis, prevention of anterior wall thinning, and attenuation of cardiac adverse remodeling (He et al., 2010). A recent in vitro study by Lin et al. (2016) proposes a cardiac fibroblast-related explanation of the multifaceted effects of sFRP2, so it cannot be excluded that the aforementioned sFRP2-mediated effects are due to its impact on cardiac fibroblasts and not noncardiac fibroblast cell types.

Schumann et al. (2000) were the first to report differences in sFRP isoform expression in human failing hearts, with 2- to 3-fold upregulated mRNA levels for the endogenous WNT signaling antagonists sFRP3 and sFRP4 (which happen to be proapoptotic) but not for sFRP1 and sFRP2. In addition, $\beta$-catenin levels were found to be decreased in the failing hearts compared with nonfailing ones, all contributing to decreased $\mathrm{WNT} / \beta$-catenin signaling of hearts progressing to $\mathrm{HF}$. Askevold et al. (2014) reported a positive correlation between increased mRNA and circulating serum levels of sFRP3 in failing hearts compared with controls. From the GISSI-HF study they included $153 \mathrm{HF}$ patients and showed that increased circulating sFRP3 levels were associated with adverse outcomes in HF. Intriguingly, a more recent study by Askevold et al. (2015) with a larger patient population showed that there might actually be a nonlinear association between circulating sFRP3 levels and outcomes. The authors demonstrate that mid-tertile serum concentrations of sFRP3 are correlated with reduced mortality in a population of old HF patients. This finding could mean that too low or too high sFRP3 levels might be leading to deleterious effects (associated with higher mortality) but intermediate sFRP3 levels correspond to low inflammatory response with reasonable levels of remodeling mechanisms, leading to improved cardiac function and thus leading to healthier patients. Furthermore, the discrepancy between the two studies could be explained by the fact that the population of the latter was generally older and suffering from more severe HF (as well as lower renal function). It should be noted, however, that Motiwala et al. (2014) report a lack of significance of sFRP3 with the progression and complications of HF. Lastly, it should be mentioned that sFRP appears to mediate the lamin $\mathrm{A} / \mathrm{C}$ gene ( $L M N A$ )-related cardiomyopathy, a condition that leads to LV dysfunction and predisposition to HF. Six-month-old Lmna ${ }^{H 222 P / H 222 P}$ mice feature elevated sFRP1 and sFRP2 transcripts (as well as upregulated DKK3 mRNA expression) and robustly decreased $\beta$-catenin protein expression, displaying the deleterious effects of WNT signaling abnormal suppression (Le Dour et al., 2017).

2. Cytoplasmic Signaling Components-DVL and GSK3 3 . The group of Hardt demonstrated a positive association between overexpression of DVL1, HF development, and increased mouse mortality, which is mediated via both $\beta$-catenin-dependent and -independent signaling pathways (Malekar et al., 2010; Zhang et al., 2015c). Both studies are covered in-depth in section VII.D.

Double KO of GSK3 $\alpha$ and GSK3 $\beta$ exhibits disastrous effects on the mouse myocardium. As reported by a recent study of Zhou et al. (2016), adult cardiomyocytes with double GSK3 deletion feature DNA damage and apoptotic cell death linked to mitotic catastrophe. This appears to be contributing to the in vivo exacerbated pathologic hypertrophy and fibrosis, leading to acute $\mathrm{HF}$ and death around 40 days of life. Several other studies have focused on manipulations via GSK3 $\beta$ signaling; however, a large part of them does not appear to be mechanistically related to WNT signaling but to act mainly via the PI3K/Akt pathway (Haq et al., 2001; Yao et al., 2008; Braz et al., 2009). Kirk et al. (2014) demonstrated that cardiac resynchronization therapy reactivates GSK3 $\beta$ in tachypacing-induced $\mathrm{HF}$ (200 beats/min) in dogs and enhances myofilament and contractile function. The report of Braz et al. (2009) was in the opposite direction. The authors showed that a GSK $3 \beta$ inhibition (leading to an activation of WNT/ $\beta$-catenin signaling) could exhibit anti-HF beneficial effects as shown in recovering dog hearts following cardiac resynchronization therapy. Once again, there is no indication that WNT signaling is heavily involved in any of these effects. Lastly, transgenic overexpression of GSK3 $\beta$ followed by TAC operation was shown to drive deleterious effects on cardiac 
function and progress to $\mathrm{HF}$ in the $\mathrm{Tg}$ mice (Hirotani et al., 2007). Recovery of this was correlated with increased phosphorylation of GSK3 $\beta$ (leading to its inhibition) and increased levels of $\beta$-catenin.

3. Signaling Inside the Nucleus- $\beta$-Catenin. Very recently, Hou et al. (2016) reported interesting evidence relating to the activation of WNT signaling in human (ischemic heart disease and idiopathic dilated cardiomyopathy) and murine (desmin-related cardiomyopathy model) failing hearts. In all three cases, $\beta$-catenin nuclear accumulation was evident, paralleled by activation of TCF7L2 and induction of c-myc mRNA transcripts. When the authors subjected cultured neonatal rat cardiomyocytes to TCF7L2 overexpression, the myocyte growth was augmented and c-myc was upregulated, whereas knockdown (via shRNA) of TCF7L2 lead to the opposite effects, indicating the positive correlation of WNT/ $\beta$-catenin signaling activation with hypertrophy and HF development. Nuclear levels of $\beta$-catenin were substantially upregulated in the myocytes of spontaneously hypertensive heart failure (SHHF) rats, whereas $\beta$-catenin presence in the intercalated disks was reduced. This latter finding is very important, because it could mean that low intercalated disk $\beta$-catenin expression is correlated with sarcomerogenesis control and the transition from compensated hypertrophy to decompensated hypertrophy and HF development (Zheng et al., 2013). A recent report of Nakagawa et al. (2016) is in the same line. Conditional gain-of-function mutation of $\beta$-catenin in endothelial cells of $\mathrm{Bmx} / \mathrm{CA}$ mice (in which $B M X-C r e E R$ controls $\beta$-catenin expression in endothelial cells) led to acute HF (with extreme dilatation and fibrosis) that is unrelated to cardiomyocytes and consequently to early mortality ( $100 \%$ of the mice die before 60 weeks of age) (Nakagawa et al., 2016). Lastly, epicardial activation of $\beta$-catenin signaling could be correlated to HF. Nuclear levels of $\beta$-catenin, LEF1, and TCF-1/3/4 are shown to be induced in the epicardium coming from end-stage pediatric allografts. Notably, CFs originating from epicardial fibrosis exhibit induced $\beta$-catenin/TCF4 axis activation, and thus epicardial $\mathrm{WNT} / \beta$-catenin could possibly be targeted to alleviate pediatric $\mathrm{HF}$ (Ye et al., 2013).

\section{F. Conclusions and Directions for Future Research}

The effect of interventions in Wnt signaling in models of cardiac disease has been the subject of extensive investigation in the last decade. From these studies it can be concluded that the expression of components of the WNT signaling pathway is modulated in a variety of cardiac pathologies, suggesting an activation of the pathway. Unfortunately, the results are often ambiguous when it comes to the question whether WNT signaling should be activated or inhibited. This is particularly the case in studies in which genetic interventions were applied. Interventions in a specific member of the large family of genes which act in WNT signaling may be compensated by the expression of other family members, hampering the interpretation of the results.

In contrast, from the studies in which pharmacological inhibitors of the pathway have been applied in models of myocardial infarction a rather clear picture is emerging: intervention at the level of either PORCPN (Bastakoty et al., 2016; Moon et al., 2017), FZD (Laeremans et al., 2011; Uitterdijk et al., 2016), the $\beta$-catenin destruction complex (Murakoshi et al., 2013), or $\beta$-catenin-mediated gene transcription (Sasaki et al., 2013) all have shown beneficial effects on infarct size and, in most cases, on cardiac function. Unfortunately, the numbers of studies in which pharmacological interventions have been applied in other models of cardiac disease are too small to draw firm conclusions, so it would be desirable to test the available compounds in models of cardiac hypertrophy and arrhythmias. Moreover, little is known about the optimal level where the pathway should be targeted: Upstream targeting (at the level of WNT production or interaction with the receptor) can target both $\beta$-catenin-dependent and -independent signaling, whereas downstream targeting (at the level of signal transduction) could introduce selectivity for a specific signaling pathway. To clarify this issue, future research should concentrate on interventions at different levels of the pathway in a single experiment to compare the effects on infarct composition and cardiac function. These studies should pay specific attention to the induction of cardiac renewal as a potential mechanism for the beneficial effects of WNT inhibition.

\section{WNT Signaling in Stem Cells}

One of the unique features of stem cells is their dual ability to self-renew as well as differentiate into cells of different lineages. Stem cells can have varying degrees of potency ranging from a pluripotent cell that is able to give rise to all three germ layers to a more limited tissue progenitor capable of generating a specific target cell (e.g., myeloid progenitor in blood). The potency of progenitors during embryonic development as well as in the adult animal is regulated by a wide range of celldependent and cell-autonomous factors. Circulating and local signals are known to affect the self-renewal and lineage specification of progenitors in a niche. WNT signaling is active in stem cell niches and can regulate the potency, fate, and renewal of progenitors. WNTs can regulate pluripotency (Willert et al., 2003; Sato et al., 2004; Dravid et al., 2005) and direct fate specification of pluripotent cells. For instance, WNTs can regulate pluripotency of embryonic stem cells but can also direct specification of germ cell lineage from the embryonic stem cells. Thus WNTs can promote the maintenance of stem cell potency or renewal or can drive differentiation 
of progenitors to adopt specific fates in a contextdependent manner. This seemingly dual function of the WNT signaling of maintaining supporting stem cell pluripotency as well as enhance differentiation can be explained at least partially by different coactivator usage (Miyabayashi et al., 2007).

\section{A. WNT Signaling Controls Stem Cell Differentiation}

In the adult mammal, stem cells or progenitors are thought to reside in specialized microenvironments called niches. Signaling within a niche determines the fate of a stem cell to self-renew or differentiate into target parenchymal cells of that tissue. An understanding of signaling pathways thus provides critical insight into mechanisms of stem cell maintenance or exhaustion. WNTs are an important family of proteins that regulate the biology of stem cells and determine cell fate decisions. WNTs can regulate differentiation of progenitors to various directions, including neuronal, hepatic, myogenic, chondrongenic, osteogenic, adipogenic, and cardiogenic cell fate (Visweswaran et al., 2015). It has been shown that WNT signaling promotes neurogenic differentiation. More specifically, in cortical mouse neural precursor cells, WNT7A promoted neuronal differentiation (Hirabayashi et al., 2004). Furthermore, WNT5A appears to drive neuronal differentiation via $\mathrm{FZD}_{3}$ and ${ }_{-5}$, and signaling through the WNT5A-JNK pathway (Jang et al., 2015). WNT activation can also enhance myogenic differentiation. Various WNT molecules have been shown to promote myogenic markers, with WNT11 being the strongest (Belema Bedada et al., 2005). Hepatic differentiation appears to be influenced by WNT signaling as well. In humans, depending on the stem cells source, the WNT signaling pathways can have different effects. They are activated in hepatic differentiation of embryonic stem cells, but downregulated in hepatic differentiation of adipose tissue-derived stem cells (Heo et al., 2013). Additionally, WNT signaling regulates stem cell differentiation in osteogenic and chondrogenic lineages as well. The data on the WNT pathways on bone development have been contradictory, with reports suggesting enhancing as well as inhibitory effects (Visweswaran et al., 2015).

With regard to WNTs in stem cell differentiation into cardiac lineage, the data suggest that they play a critical role as mentioned in detail in section IX.C. It is important to highlight that the WNT/ $\beta$-catenin pathway regulates the early differentiation of murine embryonic stem cells (Anton et al., 2007). Furthermore, regulation of WNT3A transcription serves as a turning point for cardiomyogenic differentiation (Deb et al., 2008).

\section{B. WNT Signaling and Cardiovascular Regeneration in Lower Vertebrates}

The remarkable cardiac regeneration ability in the lower vertebrates, including frogs, newts, and axolotls, was initially studied more than 50 years ago (Rumyantsev, 1966; Rumyantsev and Marakjan, 1968). More recent studies provided evidence of full regeneration of the adult hearts in newts over a period of 60 days (Witman et al., 2011). Although earlier studies showed only limited regenerative capacity, those studies were likely prematurely terminated for analysis (Porrello and Olson, 2014). In addition, the location of the injury could suggest a differential regenerative capacity of the progenitor cells or cardiomyocytes. However, zebrafish show an exceptional regenerative capacity following different modalities of cardiac injury (Kikuchi and Poss, 2012). The different modalities include surgical removal of $20 \%$ of the apex of the ventricle, cryoablation-induced injury of $25 \%$ of the apex of the ventricle, or ablation of up to $70 \%$ of cardiomyocytes by transgenic expression of diphtheria toxin (Poss et al., 2002; Chablais et al., 2011; Wang et al., 2011a). Regardless of the type of injury, the cellular mechanisms involved in heart regeneration appear to be closely similar, regardless of the injury models. The epicardium appears to be essential for these cellular changes to occur. Interestingly, genes that are normally expressed during development, such as Wilms' tumor $1 \mathrm{~b}$, appear to be upregulated during the regeneration process. Although there is ample evidence of the role of WNT signaling pathways participating in mammalian heart regeneration and remodeling, little is known regarding their role heart regeneration in lower vertebrates. WNT $/ \beta$-catenin signaling appears to be upregulated following ventricular regeneration after resection (Stoick-Cooper et al., 2007). In addition, a WNT target gene is also activated in the zebrafish after injury (Kizil et al., 2009). However, further studies are needed to elucidate the role of WNT signaling in lower vertebrate heart regeneration.

\section{WNT Signaling in Cardiomyocyte Differentiation of Stem / Progenitor Cells}

As mentioned in section VI, the studies on WNT signaling during cardiac development in vertebrates have yielded mixed results. In chicken and frog embryo experiments, the activation of $\beta$-catenin-dependent WNT signaling inhibited cardiac differentiation and WNT inhibitors had the opposite effect (Marvin et al., 2001; Schneider and Mercola, 2001). In addition, application of $\mathrm{LiCl}$, an activator of $\beta$-catenin-dependent WNT signaling, increased the myogenic differentiation of mesenchymal stem cells derived from cardiac patients (Brunt et al., 2012). Non- $\beta$-catenin-mediated signaling plays also a role in promoting cardiomyocyte differentiation. Mesenchymal stem cells transduced with WNT11 appeared to upregulate GATA-4, likely through signaling via PKC or JNK pathway (He et al., 2011). From a developmental standpoint, these insights have been applied in other projects with a direct potential clinical impact. It has been proposed that 
human embryonic stem cells could serve as a source of cardiomyocytes. Subsequently, cultured embryonic stem cells form clusters called embryonic bodies that appear to differentiate in random fashion, even to a few beating cardiomyocytes. However, addition of WNT3A to these cultures significantly increased the onset of beating activity and expression of cardiac genes (Kwon et al., 2007). Furthermore, addition of a WNT signaling inhibitor resulted in complete abolition of the beating embryonic bodies (Kwon et al., 2007). These findings suggest that $\beta$-catenin-dependent WNT signaling not only drives the cardiomyocyte formation in embryonic stem cells but also is a necessary pathway for such events.

\section{WNT Signaling in Cardiac Fibrosis and Repair}

The importance of WNT signaling in development cannot be fully appreciated without elaborating on their role in cardiac repair and fibrosis (Deb, 2014). As discussed in detail in section VIII.A, expression of several WNTs, such as WNT1, -2, -4, -7A, -10B, and -11 increases significantly shortly after an ischemic cardiac injury (Kobayashi et al., 2009; Aisagbonhi et al., 2011; Duan et al., 2012). The effects of WNT/ $\beta$-catenin signaling appears to be profibrotic and antagonism of this pathway with sFRPs appears to have beneficial effects on fibrosis, cardiac function, and left ventricular remodeling (Kobayashi et al., 2009). However, the specific mechanisms of beneficial effects of WNT antagonism following injury remains unclear, although it is tempting to speculate that this induces cardiac regeneration. Which cell populations express different WNTs in the injured hearts and how WNT/FZD signaling changes in different cell populations after cardiac injury will need to be further studied, especially to develop therapeutic strategies in the ischemic heart. Cardiac hypertrophy, in contrast to myocardial infarction, is characterized by an increase in cardiomyocyte cell size and increase fibrosis as well. Gene expression of WNTs does not appear to be altered after cardiac hypertrophy (ter Horst et al., 2012), but when patients with a hypertrophic heart develop heart failure, WNT signaling appears to be depressed (Kobayashi et al., 2009). In addition, $\beta$-catenin loss-of-function mutations in the myocytes have been associated with enhanced cardiac function, and gain-of-function mutations have been associated with reduced cardiac function resembling dilated cardiomyopathy (Baurand et al., 2007; Zelarayan et al., 2008). These findings suggest that WNTs signaling in cardiac myocytes promotes cardiac hypertrophy and suggests that inhibition of the $\beta$-catenin-dependent WNT pathway signaling might be a pharmacological target for cardiac hypertrophy.

\section{E. Conclusions and Future Perspectives}

Although there is ample evidence for the involvement of WNT signaling in stem cell homeostasis, e.g., in the intestine, its role in cardiovascular stem cell regulation is only beginning to be explored. Because regeneration of heart tissue would be a major step forward in the treatment of damage, e.g., caused by cardiac ischemia, further exploration of interventions in Wnt signaling aiming at the regeneration of heart tissue will be an exciting area of research for the near future. However, it will be challenging to specifically address the regeneration process in the heart, without affecting stem cells in other organs. A detailed molecular analysis of the signaling pathways involved in cardiac regeneration is needed to identify targeting points that are sufficiently specific to avoid unacceptable side effects in other stem cell populations.

\section{Crosstalk between WNT Signaling and Other Signal Transduction Pathways}

\section{A. Transforming Growth Factor $\beta$}

Rather than operating in isolation, WNT signaling appears to be strongly intertwined with other signaling pathways, including the TGF $\beta / \mathrm{BMP}$ and YAP/TAZ signaling. Multiple examples of crosstalk between these pathways can be found, and this crosstalk appears to be taking place at different levels in the signaling cascade (Guo and Wang, 2009). Activation of this signaling network is particularly relevant for the control of fibrosis of different organs, where differentiation of effector cells from different origins into myofibroblasts plays a key role (Piersma et al., 2015). Here we will discuss this crosstalk at multiple levels: the extracellular level, at the intracellular signal transduction, and in the nucleus.

1. Extracellular Crosstalk between WNT and TGF $\beta$ Signaling. There are multiple studies in which the mutual regulation of the expression of WNTs and TGF $\beta$ were demonstrated (van den Bosch et al., 2016). In a model of experimental autoimmune myocarditis, the myofibroblast differentiation and subsequent cardiac fibrosis induced by TGF $\beta$ was shown to be dependent on the rapid secretion of WNT proteins in a TGF $\beta$-activated kinase-1 (TAK-1)-dependent fashion (Blyszczuk et al., 2017). An increase in WNT production was also observed in TGF $\beta$-stimulated vascular smooth muscle cells overexpressing the intracellular signal transduction molecule SMAD3 (DiRenzo et al., 2016). A similar induction of WNT5A and WNT11 expression by TGF $\beta$ was recently reported in fibrotic liver and lung smooth muscle cells, respectively (Kumawat et al., 2016; Beljaars et al., 2017). However, the crosstalk between WNT and TGF $\beta$ can also follow the opposite direction as shown by the WNT3A-induced expression of TGF $\beta$ in mouse fibroblasts (Carthy et al., 2011).

2. Crosstalk at the Level of the Signal Transduction. $\mathrm{TGF} \beta$ signals through $\mathrm{TGF} \beta$ type I and II receptors, members of the tyrosine kinase receptor family. Upon binding of a TGF $\beta$ homodimer, a heterodimeric receptor 
complex is formed that can phosphorylate the signaling modulator Smad2/3. These Smads can in turn associate with Smad4, travel to the nucleus, and form a transcription module with multiple cofactors including p300 and CBP. Smad3 was found to facilitate the rapid nuclear translocation of $\beta$-catenin upon $\mathrm{TGF} \beta$ stimulation of mesenchymal stem cells (Jian et al., 2006). On the other hand, the transcriptional activity of Smad3 was significantly enhanced in cardiac fibroblasts that are conditionally depleted of GSK3 $\beta$ using the tamoxifen-inducible Col1a2-cre model (Lal et al., 2014). A direct interaction of Smad3 with a complex of axin and GSK3 was shown to result in the phosphorylation of Smad3 at $\mathrm{Thr}^{66}$, triggering its degradation via the ubiquitin/proteasome pathway (Guo et al., 2008). These observations suggest a mutual interaction between the two pathways.

The complexity of this interaction is further enhanced by the involvement of a third pathway, YAP/TAZ, that has been shown to be strongly intertwined with both TGF $\beta$ and WNT signaling in the differentiation of myofibroblasts (Piersma et al., 2015). YAP and TAZ are two effectors of Hippo signaling, another highly conserved signaling pathway involved in the regulation of heart size and stem cell fate (Xiao et al., 2016). YAP has been shown to interact with Smad7, an inhibitor of the intracellular $\mathrm{TGF} \beta$ signaling, thereby repressing the signaling to the nucleus (Piersma et al., 2015). Moreover, in the WNT off state YAP and TAZ are associated with the $\beta$-catenin destruction complex where they facilitate the ubiquitination of $\beta$-catenin by $\beta$-TrCP. Activation of WNT signaling results in dissociation of YAP and TAZ from the destruction complex and release of $\beta$-catenin (Azzolin et al., 2014). TAZ was also reported to be able to bind to DVL, thereby attenuating the activation of WNT signaling and to associate directly with $\beta$-catenin, thereby inhibiting its nuclear localization (Imajo et al., 2012).

3. Nuclear Crosstalk between WNT, TGF $\beta$ and YAP/ TAZ Signaling. A specific feature of these three signaling pathways is that they rely on the nuclear translocation of their intracellular signaling proteins to activate gene transcription. As indicated in section V.B.4, $\beta$-catenin does not possess DNA binding capabilities. The same holds true for YAP/TAZ: both form transcriptional modules with more general transcriptional coactivators such as p300 and/or CBP. Interestingly, YAP/TAZ transcription factors can be added to the TGF and WNT complexes, giving rise to additional or even synergistic activation of the gene expression. This crosstalk appears to be of specific importance in EMT and myofibroblasts activation (Piersma et al., 2015).

\section{B. Renin Angiotensin System}

Besides its central role for cardiovascular homeostasis, the renin angiotensin system (RAS) is also involved in other organ systems and is interconnected to many signaling systems both in physiologic and pathologic conditions (de Gasparo et al., 2000; Bader and Ganten, 2008).

AngII, the main effector of the renin angiotensin system, is formed by the enzymatic degradation of angiotensinogen by renin and angiotensin converting enzyme, respectively. AngII has been shown to modulate the mRNA expression of $\mathrm{FZD}_{2}$ in rat vascular smooth muscle cells in a time-dependent manner (Castoldi et al., 2005). The initial rapid upregulation of $\mathrm{FZD}_{2}$ after AngII stimulation was accompanied by a rapid upregulation of TGF $\beta$-1, suggesting a potential crosstalk between AngII and $\mathrm{FZD}_{2}$, most probably indirectly via the contribution of TGF $\beta-1$ signaling (see section $X-A$ ) (Castoldi et al., 2005). Following this early finding, more direct evidence linking the RAS to WNT signaling was identified more recently.

The (pro)renin receptor (PRR), one of the latest identified RAS family members, is able to bind renin and its precursor prorenin. Shortly after its discovery, PRR was recognized as the full-length form of a smaller protein described earlier, associated with the V-ATPase (vacuolar $\mathrm{H}^{+}$-ATPase), a multiprotein complex involved in the acidification of vesicles (Nguyen, 2011). More recently PRR was proposed to be a component of the WNT receptor complex independently of its function for renin. PRR seems to act as an adaptor between WNT receptors and the V-ATPase complex (Buechling et al., 2010; Cruciat et al., 2010). Coimmunoprecipitation experiments showed that PRR could bind $\mathrm{FZD}_{2}, \mathrm{FZD}_{8}$, and LRP6. In addition, loss of function and pharmacological inhibition of V-ATPase in vitro and in vivo revealed its necessity for $\mathrm{WNT} / \beta$-catenin signaling (Cruciat et al., 2010). Results from this study have thus shed light on an unsuspected role for PRR/V-ATPase and acidification during WNT/ $\beta$-catenin signaling. The authors hypothesized that the WNT/PRR complex is internalized upon stimulation of the FZD receptor, followed by an acidification of the endocytosed complex that allows the phosphorylation of LRP6 and the subsequent $\beta$-catenin activation (Cruciat et al., 2010). In addition to its involvement in the $\mathrm{WNT} / \beta$-catenin pathway, the PRR has been identified to be also part of the WNT/PCP pathway in Drosophila (Buechling et al., 2010; Hermle et al., 2010).

Recent in vitro and in vivo results have identified that multiple RAS genes encoding angiotensinogen, renin, angiotensin-converting enzyme, and the receptors for AngII are downstream targets of $\mathrm{WNT} / \beta$-catenin signaling. The overexpression of $\beta$-catenin or different WNT ligands upregulates the expression of RAS genes, whereas blockade of WNT/ $\beta$-catenin signaling with a small molecule inhibitor effectively downregulates RAS genes. In vivo, the inhibition of the $\mathrm{WNT} / \beta$-catenin/RAS axis was able to reduce renal inflammation and fibrosis, thus opening new therapeutic avenues (Zhou et al., 2015). 


\section{Vascular Endothelial Growth Factor}

Vascular endothelial growth factors (VEGF-A, VEGF-B, VEGF-C, and VEGF-D) are key players influencing angiogenesis through VEGF receptors (VEGFR1, VEGFR2, and VEGFR3). This VEGF system has many sites of interaction with other signaling systems such as TGF $\beta$, Notch, and FGF (Holderfield and Hughes, 2008; Dejana, 2010; Lieu et al., 2011). Studies performed in the last decade have also revealed synergistic or antagonistic interactions with WNT signaling.

The interaction between VEGF and WNT signaling was first identified in colonic neoplasia (Zhang et al., $2001)$. Similarly, the importance of the $\mathrm{WNT} / \beta$-catenin signaling pathway in VEGF signaling has also been evidenced in human retinal pigment epithelial cells subjected to hypoxia. In this model, angiogenesis induced by tissue factor was decreased in presence of the $\mathrm{WNT} / \beta$-catenin signaling pathway inhibitor IWR-1endo (Wang et al., 2016c).

Later, researchers performed a high-throughput RNA interference screen to identify novel modulators of the WNT/ $\beta$-catenin pathway and thus potential new therapeutic targets (Naik et al., 2009). A library of siRNAs was tested in a luciferase assay for activation of $\mathrm{WNT} / \beta$-catenin transcription with WNT-stimulated human cells. Follow up studies with the best hits identified VEGFR1 (but not VEGFR2 or VEGFR3) as a positive regulator of WNT signaling (Naik et al., 2009). The study showed that this VEGFR1-WNT synergy was independent of GSK3 $\beta$. A VEGFR1tyrosine phosphorylation of $\beta$-catenin itself was proposed by the authors as the mechanism for this synergistic effect (Naik et al., 2009).

Further evidence for a crosstalk between WNT and VEGF signaling was obtained in the field of developmental angiogenesis. Zebrafish embryos deficient for Rspo1 or its receptor Kremen 1 exhibited primary vessels resulting from vasculogenesis, but did not demonstrate further vascular development (Gore et al., 2011). This was found in association with a decreased expression of VEGFC. Additional experiments revealed that the expression of VEGFC was dependent of Rspo1 and WNT and that the proangiogenic effects of Rspo1/WNT signaling are mediated by VEGFC/VEGFR3 signaling (Gore et al., 2011). At an earlier developmental stage, it has been shown that VEGF signals through the WNT/ $\beta$-catenin pathway to favor the vasculogenic differentiation of mesenchymal stem cells (Zhang et al., 2016c).

Although WNT signaling is well known for its contribution to vascular development, its interplay with VEGF signaling remains poorly investigated. Additional studies are required to confirm the previous observations and to further decipher the complex WNT-VEGF interactions.

\section{Conclusions and Future Perspectives}

It is becoming increasingly clear that signaling pathways do not operate in isolation but are in fact strongly intertwined. The WNT signaling pathway appears to be no exception, as illustrated by its interactions with TGF $\beta$ signaling, the RAS and VEGF described in this section. It is quite conceivable that some of the effects that we observe by a therapeutic intervention in, e.g., the RAS are in fact the result of inhibition of an interacting signaling pathway. Although these interactions add significantly to the complexity of the study of WNT signaling, they are important because they can be a source of adverse effects of interventions. This calls for a more holistic approach in the preclinical phase of drug discovery in general to reduce the number of drugs that fail in clinical trials.

\section{Therapeutic Interventions}

Because of the involvement of WNT signaling in conditions varying from cancer to neurodegenerative diseases, many compounds have been developed that target the WNT signal transduction pathway at different levels. Although many of these compounds are aiming at the $\mathrm{WNT} / \beta$-catenin branch of the signaling, other compounds act more upstream at the level of WNT synthesis/secretion or at the receptor complex. For an overview of the sites of interaction, please see Fig. 8. The interventions are schematically represented in Tables 2-5.

\section{A. WNT Synthesis-Secretion}

The first level of the WNT signaling cascade is undoubtedly the production and secretion of the WNT ligand. Following its production, WNT is not released as is, but undergoes glycosylation and palmitoylation, two processes which modify WNT and are indispensable for the secretion of the active WNT ligand (Komekado et al., 2007; Kurayoshi et al., 2007). PORCN, residing in the endoplasmic reticulum of WNT-producing cells, is a critical factor in this transformation and secretion of WNTs (Komekado et al., 2007). A wide range of PORCN inhibitors has been developed with demonstrated effects on WNT secretion, thus mediating the prevention of WNT signaling activation. Additionally, as shown by in vivo studies with transgenic mice, genetic loss of Porcn leads to dramatic inhibition of endogenous WNT production, but mice can still respond to exogenous WNTs, indicating that the receptor compartment is not affected (Barrott et al., 2011). Studies with human astrocytes showed that involvement of PORCN in the WNT production might be cell type and WNT subtype dependent (Richards et al., 2014). Chen et al. (2009a) were the first to show that four compounds, named IWP1 to -4 (inhibitors of WNT production), can attenuate the 


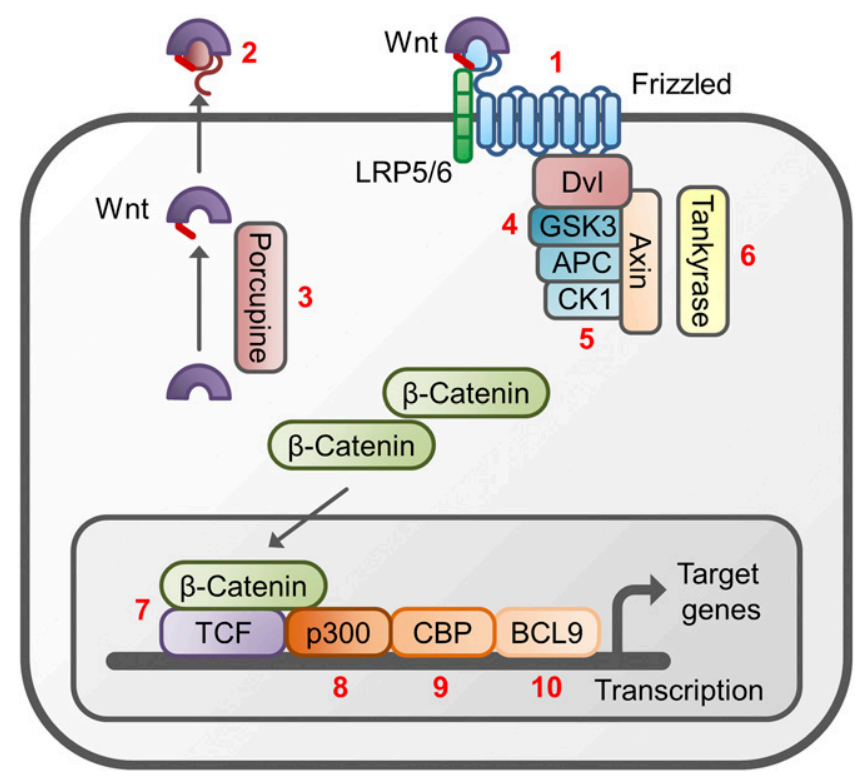

Fig. 8. Different sites of pharmacological intervention in WNT signaling. Details are provided in section XI. (1) Targeting of FZD proteins (e.g., vantictumab or UM206); (2) WNT scavengers (e.g., ipafricept); (3) Porcupine inhibitors (e.g., LGK974 or IWP-2); (4) Glycogen synthase kinase-3 $\beta$ (GSK3 $\beta$ ) inhibitors (e.g., LiCl, valproic acid, 6-BIO); (5) Casein kinase-1 (CK1) inhibitors (e.g., pyrvinium); (6) Tankyrase inhibitors (e.g., XAV939, IWR-1); (7) Inhibitors of the interaction between $\beta$-catenin and the TCF transcription factors (e.g., ICRT3, -5 , and -14 , nonsteroid antiinflammatory drugs); $(8,9,10)$ Inhibitors of the interaction of the transcription complex with the cofactors p300, CBP, and BCL9 (e.g., windorphen, ICG-001, and SAH-BCL9).

secretion of the WNT ligands. These four IWP molecules induce PORCN-mediated inhibition of WNT 1, 2, and 3a production. A few years later they reported another 13 IWP Porcn inhibitors and attention was drawn toward IWP-L6, which was shown to be 100 times more potent than the originally designed IWP-2 (Wang et al., 2013b). In addition, LGK974, a potent and specific PORCN-inhibitor, was first discovered by the research group of Harris; the compound is also referred to as WNT974. The orally bioavailable molecule inhibits PORCN, as well as operating more downstream too by blocking WNT-mediated LRP5/6 phosphorylation, and is very effective against various types of cancer both in vitro and in vivo (Liu et al., 2013). Interestingly, LKG974 can work in synergy with the PI3K inhibitor GDC-0941 and produce antitumor effects in MDA-MB231 breast cancer cells (Tzeng et al., 2015). Furthermore, LGK974 has also entered a phase I clinical trial (https://clinicaltrials.gov/ct2/show/NCT01351103), including patients with colorectal cancer, pancreatic adenocarcinoma, and other cancer types with wellestablished association with the WNT signaling cascade. The beneficial effects of this compound, as well as the PORCN inhibitor GNF-6231, on infarct healing was previously discussed (section VIII.A.1). Quite recently, ETC-159 was demonstrated to strongly inhibit PORCN, block WNT secretion, attenuate the expression of the $\beta$-catenin target genes TCF7, c-myc and axin2, and to possess robust antitumor capabilities versus teratocarcinomas and colon cancers, as well as preventing remissions (Madan et al., 2016a). Proffitt et al. (2013) propose the compound WNT-C59 as a robust and safe inhibitor of PORCN. All PORCN isoforms and all WNT members that can activate the $\beta$-catenin-dependent pathway were reported to be substantially inhibited by WNT-C59 and this is reflected by its excellent antitumor effects in mice, where it prevents MMTVWNT1 growth (Proffitt et al., 2013). WNT-C59 has the additional advantage of being orally bioavailable. Treatment with WNT-C59 leads to the robust attenuation of renal fibrosis in a unilateral ureteral obstruction mouse model. This is effectuated by a complex crosstalking mechanism and since the disruption of porcupine leads to blunted WNT secretion, this has the inhibition of inflammatory cytokine activation and thus a suppression of ECM-depositing fibroblasts expression as a consequence (causing an attenuation in fibrosis) (Madan et al., 2016b). Novel PORCN inhibitors, such as compound 53 have shown promising preliminary results, but their physicochemical and pharmacokinetic properties restrict their applicability. Thus, more research is required for their improvement, before they are further studied in vitro and in vivo (Xu et al., 2016). Lastly, one very important point that should be taken into consideration when using factors acting at the level of PORCN is their relative nonselectivity. Targeting PORCN usually leads to a global WNT secretion suppression, both the ones forming the $\beta$-catenindependent pathway (WNT1, $-2,-3,-8 \mathrm{~A} / \mathrm{B}$, and $-10 \mathrm{~A} / \mathrm{B}$ ), as well as the $\beta$-catenin-independent WNTs- $4,-5 \mathrm{~A} / 5 \mathrm{~B}$, $-6,-7 \mathrm{~A} / \mathrm{B}$, and -11 ), thus there is always the danger for off-target effects.

WNT16 expression can be directly affected by a compound called 4,4-di-O-methylellagic acid (4,4-DiOMEA). The expression of WNT16 mRNA levels is correlated with concentrations of 4,4-DiOMEA, and this molecule exhibits robust antiproliferative effects on colon cancer cell lines, even ones that are resistant to well-established chemotherapy drugs such as 5-FU (Ramirez de Molina et al., 2015).

\section{B. Extracellular Pharmacological Targeting of WNT Signaling: WNT-FZD Interaction}

The interaction of the WNT ligands with their corresponding FZD receptors is the hallmark event initiating the signal transduction. A long list of compounds have been tested and have been found to influence the WNT-FZD interaction, whereas a probably even longer list has been identified via high-throughput methods (Koval and Katanaev, 2012) and awaits further investigation. As discussed in section III.E, the interaction of WNT and FZD components is complex. The scientific community has been assuming a rather simplified view of the kinetics, the selectivity of some WNTs for some FZD, the nature and varied types of 
TABLE 2

Interventions in WNT synthesis and secretion

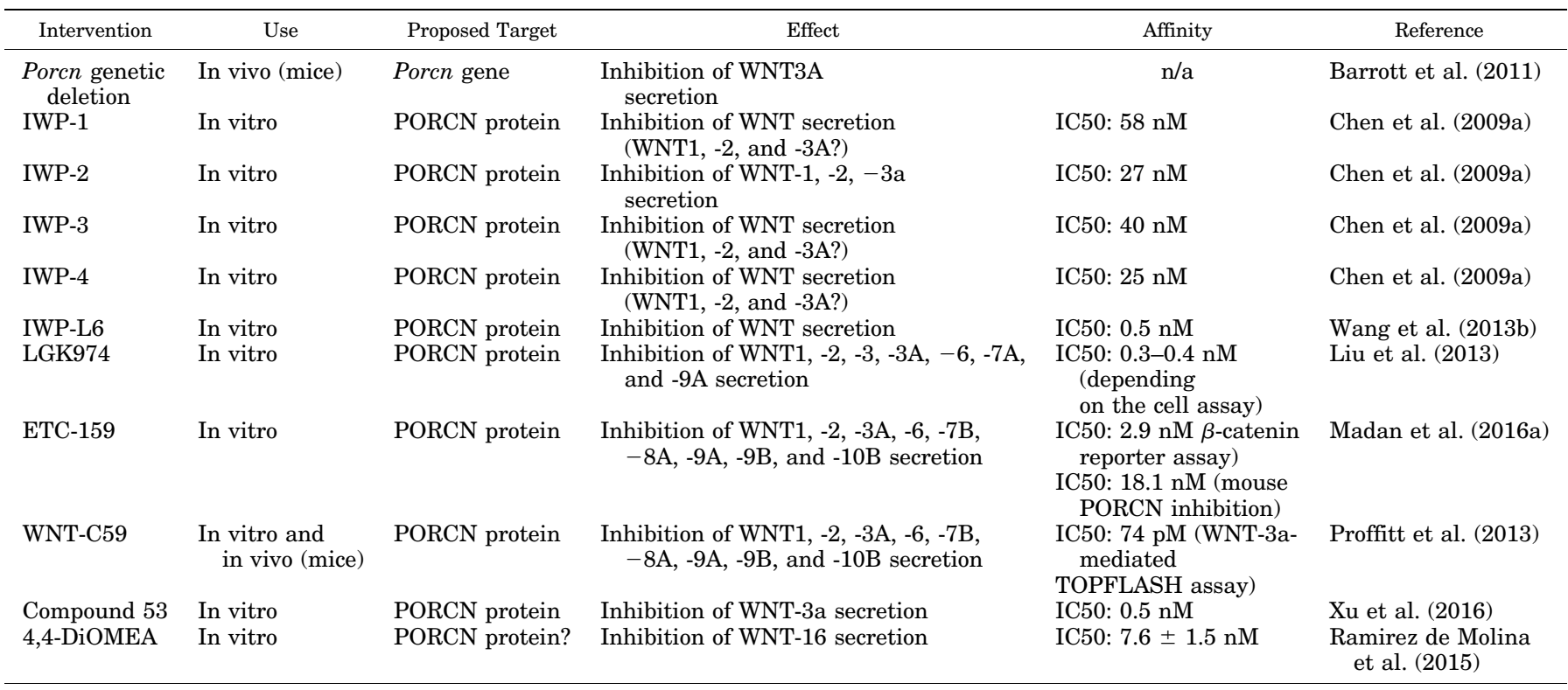

complexes formed, the cofactors playing secondary roles in the complex formation, the role of G proteins, etc. Nevertheless, it appears that our knowledge is far from complete and more research is needed in our understanding of receptor complex formation. Thus, one should make sure to take into account all factors playing a major role in the formation of the WNT-FZD complex, before devising a plan to prevent or promote the complex formation (Schulte, 2015).

Blockade of FZD receptors with antibodies is an attractive therapeutic strategy, because antibodies usually bind their target with high affinity and selectivity. Various groups have used antibodies directed against several FZD isoforms. In a seminal study, Gurney et al. (2012) showed that the antibody OMP18R5 (vantictumab) could be a novel tool in blocking multiple FZD receptor targets. Initially, OMP-18R5 was thought to bind to $\mathrm{FZD}_{7}$ only; however, it was later shown to act antagonistically against $\mathrm{FZD}_{1},-2,-5$, and ${ }_{-8}$ as well. OMP-18R5 is effective against a wide range of human cancers (lung, pancreas, breast, teratocarcinoma), and it was shown to act synergistically with first-line chemotherapy (Gurney et al., 2012). A chimeric humanized monoclonal antibody directed against FZD $_{10}$ was developed, code-named OTSA101. The plain (nonradiolabeled) antibody is a weak antagonist of $\mathrm{FZD}_{10}$; however, when radiolabeled with Yttrium 90 (OTSA101-DTPA-90Y), it exhibits a robust antitumor effect. Even a single intravenous injection of the labeled monoclonal antibody suppressed the growth of sarcoma tumor cells in a mouse xenograft model, without leading to substantial toxicity (Fukukawa et al., 2008). Currently, OTSA101 is in Phase I clinical trial stage (https://clinicaltrials.gov/ct2/show/ NCT01469975; "SYNFRIZZ") with patients suffering from synovial sarcoma. Furthermore, Nagayama et al. (2005) described a polyclonal antibody (TT641 pAb) against $\mathrm{FZD}_{10}$ that acts on its extracellular domain. An attenuated growth of synovial sarcoma tumor cells in vitro, as well as of xenografts after injection in mice was observed using this antibody. In addition, Paes et al. (2011) generated an $\mathrm{FZD}_{4}$ monoclonal antibody (1.99.25). The antibody binds the $\mathrm{FZD}_{4} \mathrm{CRD}$ and thus neutralizes the WNT3A-mediated $\beta$-catenin signaling in a retinopathy mouse model. A polyclonal antibody directed against $\mathrm{FZD}_{5}$ has been developed, with evidence showing that it attenuates the proinflammatory cytokines IL-6, IL-15, and RANKL in rheumatoid arthritis (Sen et al., 2001), while a blocking antibody against $\mathrm{FZD}_{7}$ has been used both in vitro and in vivo in Wilm's tumor, with beneficial effects (Pode-Shakked et al., 2011).

OMP-54F28 (ipafricept) is a novel fusion protein that targets $\mathrm{FZD}_{8}$-interacting WNT ligands. It has been constructed by fusing the CRD of $\mathrm{FZD}_{8}$ with the Fc domain of immunoglobulin. The latter competes with the native $\mathrm{FZD}_{8}$ receptor for its ligands and thereby antagonizes WNT signaling (thus acting more as a scavenger of WNT ligands, rather than an antagonist of FZD). The first preclinical studies showed beneficial effects of OMP-54F28 against a variety of solid tumors (Le et al., 2015). Currently there is one clinical trial that has been completed, one is in progress, and two are recruiting patients, in the context of the use of OMP$54 \mathrm{~F} 28$ in various human cancers (https://www.clinicaltrials.gov/ct2/results?term=omp54f28\&Search=Search). The first data show beneficial therapeutic effects against ovarian cancer when the antibody is used along with paclitaxel and carboplatin (Fischer et al., 2015), whereas the antibody did not exhibit pronounced toxicity in 
TABLE 3

Extracellular targeting of the Wnt signaling pathway

\begin{tabular}{|c|c|c|c|c|c|}
\hline Intervention & Use & Proposed Target & Effect & Affinity & Reference \\
\hline OMP-18R5 (mAb) & $\begin{array}{l}\text { In vitro and in vivo } \\
\text { (mice) }\end{array}$ & $\begin{array}{l}\mathrm{FZD}_{1}, \mathrm{FZD}_{2}, \mathrm{FZD}_{5}, \\
\mathrm{FZD}_{7}, \mathrm{FZD}_{8}\end{array}$ & $\begin{array}{l}\text { Inhibition of WNT-FZD } \\
\text { interaction }\end{array}$ & $\mathrm{n} / \mathrm{a}$ & Gurney et al. (2012) \\
\hline $\begin{array}{l}\text { OTSA101 }(\mathrm{mAb})+ \\
\text { Yttrium } 90\end{array}$ & $\begin{array}{l}\text { In vitro, in vivo (mice) } \\
\text { and CT }\end{array}$ & $\mathrm{FZD}_{10}$ & $\begin{array}{l}\text { Inhibition of WNT-FZD } \\
\text { interaction }\end{array}$ & $\mathrm{n} / \mathrm{a}$ & $\begin{array}{l}\text { Fukukawa et al. } \\
\text { (2008) }\end{array}$ \\
\hline TT641 (pAb) & $\begin{array}{l}\text { In vitro and in vivo } \\
\text { (mice) }\end{array}$ & $\mathrm{FZD}_{10}$ & $\begin{array}{l}\text { Inhibition of WNT-FZD } \\
\text { interaction }\end{array}$ & $\mathrm{n} / \mathrm{a}$ & $\underset{(2005)}{\text { Nagayama et al. }}$ \\
\hline $1.99 .15(\mathrm{mAb})$ & $\begin{array}{l}\text { In vitro and in vivo } \\
\text { (mice) }\end{array}$ & $\mathrm{FZD}_{4}$ & $\begin{array}{l}\text { Inhibition of WNT-FZD } \\
\text { interaction }\end{array}$ & $\mathrm{n} / \mathrm{a}$ & Paes et al. (2011) \\
\hline pAb against FZD5 & In vitro & $\mathrm{FZD}_{5}$ & $\begin{array}{l}\text { Inhibition of WNT-FZD } \\
\text { interaction }\end{array}$ & $\mathrm{n} / \mathrm{a}$ & Sen et al. (2001) \\
\hline Ab against FZD7 & $\begin{array}{l}\text { In vitro and in vivo } \\
\text { (chick embryo) }\end{array}$ & $\mathrm{FZD}_{7}$ & $\begin{array}{l}\text { Inhibition of WNT-FZD } \\
\text { interaction }\end{array}$ & $\mathrm{n} / \mathrm{a}$ & $\begin{array}{l}\text { Pode-Shakked et al. } \\
\text { (2011) }\end{array}$ \\
\hline $\begin{array}{l}\text { OMP-54F28 fusion } \\
\text { protein }\end{array}$ & $\begin{array}{l}\text { In vitro, in vivo (mice) } \\
\text { and } \mathrm{CT}\end{array}$ & $\begin{array}{l}\mathrm{FZD}_{8} \text {-interacting } \\
\text { WNT ligands }\end{array}$ & $\begin{array}{l}\text { Inhibition of WNT-FZD } \\
\text { interaction }\end{array}$ & $\mathrm{n} / \mathrm{a}$ & $\begin{array}{l}\text { Le et al. (2015), } \\
\text { Fischer et al. } \\
(2015) \\
\text { Jimeno et al. (2014) }\end{array}$ \\
\hline UM206 & $\begin{array}{l}\text { In vitro and in vivo } \\
\text { (mice) }\end{array}$ & $\mathrm{FZD}_{1}$ and $\mathrm{FZD}_{2}$ & $\begin{array}{l}\text { Inhibition of WNT-FZD } \\
\text { interaction }\end{array}$ & $\begin{array}{l}\mathrm{IC}_{50}: 2.10 \mathrm{nM} \\
\quad \text { for } \mathrm{rFZD} 1 ; \\
\mathrm{IC}_{50}: 0.0169 \mathrm{nM} \\
\quad \text { for } \mathrm{rFZD2}\end{array}$ & $\begin{array}{l}\text { Laeremans et al. } \\
\quad(2011) \\
\text { Uitterdijk et al. } \\
\quad(2016)\end{array}$ \\
\hline $\begin{array}{l}\text { Foxy } 5+\mathrm{FZD}_{5} \\
\text { receptor-blocking } \\
\mathrm{Ab}\end{array}$ & $\begin{array}{l}\text { In vitro and in vivo } \\
\text { (mice) }\end{array}$ & $\mathrm{FZD}_{5}$ & $\begin{array}{l}\text { Mimicking of WNT5A } \\
\text { (effect independent of } \\
\beta \text {-catenin) }\end{array}$ & $\mathrm{n} / \mathrm{a}$ & $\begin{array}{l}\text { Safholm et al. (2006) } \\
\text { Safholm et al. (2008) }\end{array}$ \\
\hline Box5 & In vitro & $\mathrm{FZD}_{5}(?)$ & $\begin{array}{l}\text { Blockade of WNT5A } \\
\text { signaling }\end{array}$ & $\mathrm{n} / \mathrm{a}$ & Jenei et al. (2009) \\
\hline 3235-0367 & In vitro & $\mathrm{FZD}_{8} \mathrm{CRD}$ & $\begin{array}{l}\text { Inhibition of WNT-FZD } \\
\text { interaction }\end{array}$ & $\mathrm{IC}_{50}: 7.1 \mu \mathrm{M}$ & Lee et al. (2015) \\
\hline 1094-0205 & In vitro & $\mathrm{FZD}_{8} \mathrm{CRD}$ & $\begin{array}{l}\text { Inhibition of WNT-FZD } \\
\text { interaction }\end{array}$ & $\mathrm{IC}_{50}: 5 \mu \mathrm{M}$ & Lee et al. (2015) \\
\hline 2124-0331 & In vitro & $\mathrm{FZD}_{8} \mathrm{CRD}$ & $\begin{array}{l}\text { Inhibition of WNT-FZD } \\
\text { interaction }\end{array}$ & $\mathrm{IC}_{50}: 10.4 \mu \mathrm{M}$ & Lee et al. (2015) \\
\hline NSC36784 & In vitro & $\mathrm{FZD}_{8} \mathrm{CRD}$ & $\begin{array}{l}\text { Inhibition of WNT-FZD } \\
\text { interaction }\end{array}$ & $\mathrm{IC}_{50}: 6.5 \mu \mathrm{M}$ & Lee et al. (2015) \\
\hline NSC654259 & In vitro & $\mathrm{FZD}_{8} \mathrm{CRD}$ & $\begin{array}{l}\text { Inhibition of WNT-FZD } \\
\text { interaction }\end{array}$ & $\mathrm{IC}_{50}: 5.7 \mu \mathrm{M}$ & Lee et al. (2015) \\
\hline \multirow[t]{2}{*}{ Niclosamide } & In vitro & $\mathrm{FZD}_{1}$ (internalization) & $\begin{array}{l}\text { Inhibition of WNT-FZD } \\
\text { interaction }\end{array}$ & $\mathrm{IC}_{50}: 0.5 \mu \mathrm{M}$ & Chen et al. (2009b) \\
\hline & In vitro & LRP6 & LRP6 degradation & $\begin{array}{l}\mathrm{IC}_{50}: 0.33-0.75 \mu \mathrm{M} \\
\text { (depending on the } \\
\text { cell line) }\end{array}$ & Lu et al. (2011b) \\
\hline \multirow[t]{3}{*}{ Curcumin } & In vitro & $\mathrm{FZD}_{1}$ & $\begin{array}{l}\text { Inhibition of WNT-FZD } \\
\text { interaction (?) }\end{array}$ & $\mathrm{n} / \mathrm{a}$ & Yan et al. (2005) \\
\hline & In vitro & WNT3A; LRP6 & $\begin{array}{l}\text { Inhibition of WNT-FZD } \\
\text { interaction (?) }\end{array}$ & $\mathrm{n} / \mathrm{a}$ & Zheng et al. (2017) \\
\hline & In vitro & WNT10B; FZD ${ }_{2} ;$ LRP5 & $\begin{array}{l}\text { Enhancement of WNT-FZD } \\
\text { interaction }\end{array}$ & $\mathrm{n} / \mathrm{a}$ & Ahn et al. (2010) \\
\hline anti-Sclerostin $\mathrm{Ab}$ & In vitro & $\begin{array}{l}\text { Sclerostin binding } \\
\text { to LRP5/6 }\end{array}$ & $\begin{array}{l}\text { Prevention of Sclerostin- } \\
\text { mediated disruption } \\
\text { of LRP5/6-FZD complex } \\
\text { formation }\end{array}$ & $\mathrm{n} / \mathrm{a}$ & $\begin{array}{l}\text { van Dinther et al. } \\
\text { (2013) }\end{array}$ \\
\hline \multirow[t]{2}{*}{ Salinomycin } & In vitro & LRP6 (?) & $\begin{array}{l}\text { Inhibition of WNT1/FZD } / \\
\text { LRP6 and } \\
\text { WNT3/FZD5/LRP6 } \\
\text { complexes }\end{array}$ & $\mathrm{IC}_{50}: 163 \mathrm{nM}(\mathrm{WNT}-1)$ & Lu et al. (2011) \\
\hline & In vitro & LRP6 & $\begin{array}{l}\text { Inhibition of WNT/FZD/ } \\
\text { LRP6 complex (?) }\end{array}$ & $\mathrm{n} / \mathrm{a}$ & $\mathrm{Lu}$ and $\mathrm{Li}(2014)$ \\
\hline Leptin & In vivo (mice) & $\begin{array}{l}\text { LRP6; WNT4 and } \\
\text { WNT7 (?); } \\
\text { GSK-3 } \beta\end{array}$ & $\begin{array}{l}\text { Enhancement of WNT4/ } \\
\text { FZD/LRP6 } \\
\text { and WNT7A/FZD/LRP6 } \\
\text { complexes }\end{array}$ & $\mathrm{n} / \mathrm{a}$ & Benzler et al. (2013) \\
\hline Silibinin & In vitro & LRP6 & $\begin{array}{l}\text { Inhibition of WNT3A/FZD/ } \\
\text { LRP6 complex }\end{array}$ & $\begin{array}{l}\text { IC }_{50}: 34-122 \mu \mathrm{M} \\
\text { (depending on the } \\
\text { cell line) }\end{array}$ & Lu et al. (2012) \\
\hline \multirow[t]{2}{*}{ anti-LRP6 Abs } & In vitro & LRP6 & $\begin{array}{l}\text { Inhibition of WNT1 and } \\
\text { WNT3A-mediated } \\
\text { cascade } \\
\text { (WNT/FZD/LRP6 complex?) }\end{array}$ & $\mathrm{n} / \mathrm{a}$ & $\begin{array}{l}\text { Ettenberg et al. } \\
(2010)\end{array}$ \\
\hline & In vitro & LRP6 & $\begin{array}{l}\text { Inhibition or enhancement } \\
\text { of WNT1/2/2B/4/6/7A/7B/ } \\
\text { 8A/9B/10A/10B-mediated } \\
\text { cascade (WNT/FZD/LRP6 } \\
\text { complex?) }\end{array}$ & $\mathrm{n} / \mathrm{a}$ & Gong et al. (2010) \\
\hline
\end{tabular}


TABLE 3-Continued

\begin{tabular}{|c|c|c|c|c|c|}
\hline Intervention & Use & Proposed Target & Effect & Affinity & Reference \\
\hline 28aa peptide & $\begin{array}{l}\text { In vitro and } \\
\text { in vivo (mice) }\end{array}$ & LRP5/6 & $\begin{array}{l}\text { Mimicking of LRP5/6- } \\
\text { domain } \rightarrow \\
\text { Enhancement of } \\
\text { WNT signaling (via } \\
\text { N-cadherin) }\end{array}$ & $\mathrm{n} / \mathrm{a}$ & Hay et al. (2012) \\
\hline DKK1 and DKK2 & $\begin{array}{l}\text { In vivo (Xenopus } \\
\text { embryos) }\end{array}$ & LRP6 & $\begin{array}{l}\text { Inhibition of WNT } \\
\text { signaling }\end{array}$ & $\mathrm{n} / \mathrm{a}$ & Mao et al. (2001) \\
\hline anti-DKK1 Ab & $\begin{array}{l}\text { In vitro and } \\
\text { in vivo (mice) }\end{array}$ & DKK1 & $\begin{array}{l}\text { Neutralization of DKK1 } \rightarrow \\
\text { Enhancement of WNT } \\
\text { signaling }\end{array}$ & $\mathrm{n} / \mathrm{a}$ & Sato et al. (2010b) \\
\hline BHQ880 mAb & $\begin{array}{l}\text { In vitro, in vivo } \\
\text { (mice) and CT }\end{array}$ & DKK1 & $\begin{array}{l}\text { Neutralization of DKK1 } \rightarrow \\
\text { Enhancement of WNT } \\
\text { signaling }\end{array}$ & $\mathrm{n} / \mathrm{a}$ & Fulciniti et al. (2009) \\
\hline DKK1 vaccine & In vivo (mice) & DKK1 & $\begin{array}{l}\text { Enhancement of DKK1 } \\
\text { levels } \rightarrow \text { Inhibition of } \\
\text { WNT signaling }\end{array}$ & $\mathrm{n} / \mathrm{a}$ & $\begin{array}{l}\text { Iyer et al. (2014) } \\
\text { Qian et al. (2012) }\end{array}$ \\
\hline $\begin{array}{l}\text { Iminooxothiazolidines } \\
\text { (Compounds 1-34) }\end{array}$ & In vitro & sFRP1 & $\begin{array}{l}\text { Inhibition of sFRP1 } \rightarrow \\
\text { Enhancement of WNT } \\
\text { signaling }\end{array}$ & $\begin{array}{l}\mathrm{EC}_{50}: 7.2 \mu \mathrm{M} \\
\quad(\text { Compound 1) }\end{array}$ & Shi et al. (2009) \\
\hline WAY-316606 & In vitro & sFRP1 & $\begin{array}{l}\text { Inhibition of sFRP1 } \rightarrow \\
\text { Enhancement of WNT } \\
\text { signaling }\end{array}$ & $\begin{array}{l}\mathrm{IC}_{50}: 0.5 \mu \mathrm{M} \\
\mathrm{EC}: 0.65 \mu \mathrm{M}\end{array}$ & Bodine et al. (2009) \\
\hline $\begin{array}{l}\text { N-substituted } \\
\text { piperidinyl } \\
\text { diphenylsulfonyl } \\
\text { sulfonamides }\end{array}$ & In vitro & sFRP1 & $\begin{array}{l}\text { Inhibition of sFRP1 } \rightarrow \\
\text { Enhancement of WNT } \\
\text { signaling }\end{array}$ & $\begin{array}{l}\mathrm{IC}_{50}^{50}: 0.04-0.87 \mu \mathrm{M} \\
\text { ED50: } 0.07-1.9 \mu \mathrm{M}\end{array}$ & $\begin{array}{l}\text { Moore et al. (2010) } \\
\text { Moore et al. (2010) }\end{array}$ \\
\hline
\end{tabular}

patients with advanced solid tumors, although bone effects were observed (Jimeno et al., 2014).

Our laboratory has been active for more than a decade in developing interventions aiming at the WNT-FZD interaction. We focused on identifying molecules that block FZD proteins, thereby preventing the WNT and FZD complex formation, eventually leading to the attenuation of signal transduction. We previously showed that UM206, a 13-amino acid-long peptide fragment of WNT3A/WNT5A, can have profound effects on FZD-receptor blockade, leading to a suppression of WNT signaling pathway (Laeremans et al., 2011). UM206 possesses two Cys residues and it was shown that substituting both of them with another amino acid can have detrimental effects for the activity of the molecule, which is indicative for a possible covalent binding between the two Cys of UM206 and the Cys-rich domain of FZD. Furthermore, UM206 may be rather selective, as shown by the fact that it blocked $\mathrm{FZD}_{1}$ and $\mathrm{FZD}_{2}$ receptors in the presence of WNT3A, but not $\mathrm{FZD}_{3}$ or $\mathrm{FZD}_{4}$. Due to these pronounced effects of UM206 on the WNT signaling pathway, we decided to investigate its effects following MI in mice. UM206 treatment demonstrated beneficial effects on cardiac remodeling and prevented HF development 35 days post-MI, an outcome attributed to increased myofibroblast numbers in the infarct area and better scar healing. Reduced dilatation of the left ventricle and improved infarct healing were also observed in a pig MI model where UM206 was infused for 5 weeks post-MI, albeit that the myofibroblasts numbers were actually reduced in this study (Uitterdijk et al., 2016). It has to be noted, however, that direct binding of UM206 to the CRD of FZD has not been observed, and, given that the peptide is derived from a region of WNT5A that appears to be not in direct contact with FZD (Janda et al., 2012), it is more likely that peptide inhibits the formation of the complex of ligand and (co)-receptors in a different but yet unidentified way.

Foxy5 is a formylated hexapeptide that was shown to act via $\mathrm{FZD}_{5}$ receptors, leading to impaired migration of MDA-MB-468 breast cancer tumor cells. The authors pretreated cells with a $\mathrm{FZD}_{5}$-specific blocking antibody and observed an attenuation of the effects of Foxy5, a phenomenon implying that the compound is most probably mediating its effects via the $\mathrm{FZD}_{5}$ receptor but not via $\mathrm{FZD}_{2}$ (Safholm et al., 2006, 2008). The same group investigated later another compound called Box5, which shares the same amino acid sequence with Foxy5 but possesses a butyloxycarbonyl group (instead of a formyl group) at the $\mathrm{N}$ terminal of its hexapeptide. Box5 directly antagonizes the WNT5A-mediated cellular signaling by attenuating WNT5A-mediated $\mathrm{Ca}^{2+}$ and PKC signaling but, although it was not proven, there is the assumption that is also acts via a blockade of $\mathrm{FZD}_{5}$ receptor similar to Foxy5 (Jenei et al., 2009).

Recently, a nonpeptidergic compound was described that can interact with $\mathrm{FZD}_{4}$. The compound, named FzM1, was shown to bind to the intracellular loop three of $\mathrm{FZD}_{4}$ and blocking the Norrin-stimulated activation of TCF/LEF with micromolar affinity. Interestingly, the compound is also able to act as a chaperone for the 
TABLE 4

Interventions in the intracellular compartment

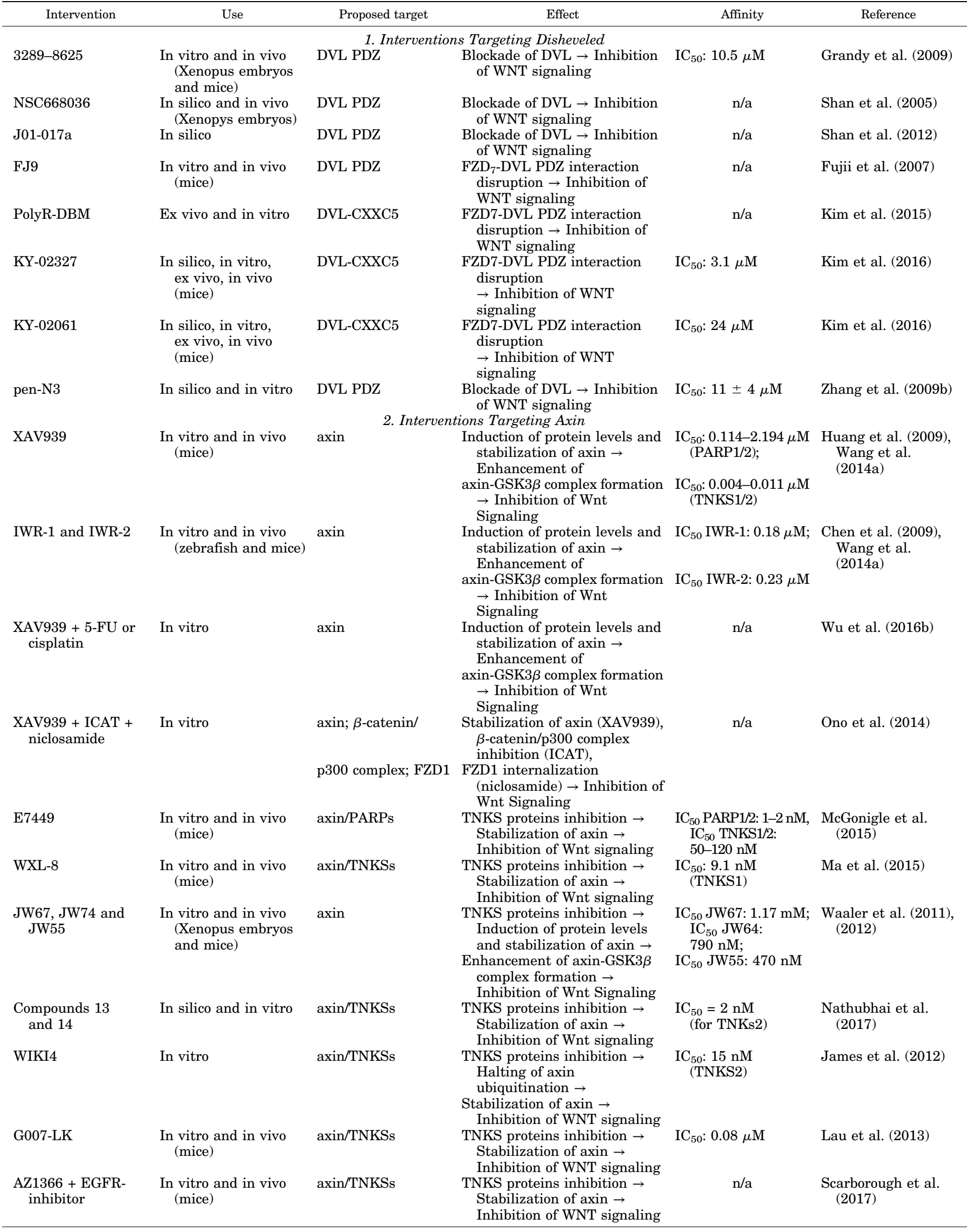


TABLE 4-Continued

\begin{tabular}{|c|c|c|c|c|c|}
\hline Intervention & Use & Proposed target & Effect & Affinity & Reference \\
\hline $\begin{array}{l}\mathrm{AZ} 1366+ \\
\quad \text { irinotecanin }\end{array}$ & $\begin{array}{l}\text { In vitro and in vivo } \\
\text { (mice) }\end{array}$ & $\operatorname{axin} / \mathrm{TNKSs}$ & $\begin{array}{l}\text { TNKS proteins inhibition } \rightarrow \\
\text { axin induction } \rightarrow\end{array}$ & $\mathrm{n} / \mathrm{a}$ & $\begin{array}{l}\text { Quackenbush et al. } \\
\text { (2016) }\end{array}$ \\
\hline IWRs (1-5) & $\begin{array}{l}\text { In vitro and in vivo } \\
\text { (zebrafish) }\end{array}$ & axin/TNKSs & $\begin{array}{l}\text { TNKS proteins inhibition } \rightarrow \\
\text { Induction of protein levels } \\
\text { and stabilization of axin } \rightarrow \\
\text { Enhancement of axin-GSK3 } \beta \\
\text { complex formation } \rightarrow \\
\text { Inhibition of Wnt Signaling }\end{array}$ & $\mathrm{IC}_{50}: 0.18-2.0 \mu \mathrm{M}$ & Chen et al. (2009) \\
\hline NVP-TNKS656 & In silico and in vitro & $\operatorname{axin} / \mathrm{TNKSs}$ & $\begin{array}{l}\text { TNKS proteins inhibition } \rightarrow \\
\text { Induction of protein levels } \\
\text { and stabilization of axin } \rightarrow \\
\text { Enhancement of axin-GSK3 } \beta \\
\text { complex formation } \rightarrow \\
\text { Inhibition of Wnt Signaling }\end{array}$ & $\begin{array}{l}\mathrm{IC}_{50}: 0.006 \mu \mathrm{M} \\
\quad(\mathrm{TNKS} 2)\end{array}$ & Shultz et al. (2013) \\
\hline Compounds 9 and 25 & In silico and in vitro & $\operatorname{axin} / \mathrm{TNKSs}$ & $\begin{array}{l}\text { TNKS proteins inhibition } \rightarrow \\
\text { Induction of protein levels } \\
\text { and stabilization of axin } \rightarrow \\
\text { Enhancement of axin-GSK3 } \beta \\
\text { complex formation } \rightarrow \\
\text { Inhibition of Wnt Signaling }\end{array}$ & $\begin{array}{l}\mathrm{IC}_{50}:<0.003 \mu \mathrm{M} \\
\quad \text { (compound 9:) } \\
\mathrm{IC}_{50}: 0.005 \mu \mathrm{M}(\mathrm{Wnt} \\
\text { cell) (compound } \\
25)\end{array}$ & $\begin{array}{l}\text { Johannes et al. } \\
\text { (2015) }\end{array}$ \\
\hline SEN461 & $\begin{array}{l}\text { In vitro and in vivo } \\
\text { (Xenopus embryos } \\
\text { and mice) }\end{array}$ & $\begin{array}{l}\text { axin } \\
\text { (not via TNKs?) }\end{array}$ & $\begin{array}{l}\text { Induction of protein levels and } \\
\text { stabilization of axin } \rightarrow \\
\text { Enhancement of axin- } \\
\text { GSK3 } \beta \\
\text { complex formation } \rightarrow \\
\text { Inhibition of Wnt Signaling }\end{array}$ & $\begin{array}{l}\mathrm{IC}_{50}: 18 \mu \mathrm{M} \\
\quad(\mathrm{TNKS} 1) ; \mathrm{IC}_{50}: \\
2.9 \mu \mathrm{M}(\mathrm{TNKS} 2) ; \\
\mathrm{IC}_{50}: 0.2-1.9 \mu \mathrm{M}(\mathrm{Ca} \\
\quad \text { cell assays) }\end{array}$ & $\begin{array}{l}\text { De Robertis et al. } \\
(2014)\end{array}$ \\
\hline $\begin{array}{l}\text { Tigecycline } \\
\text { (monotherapy or }+ \\
\text { paclitaxel) }\end{array}$ & $\begin{array}{l}\text { In vitro and in vivo } \\
\text { (mice) }\end{array}$ & $\operatorname{axin} 1$ & $\begin{array}{l}\text { Induction of protein levels and } \\
\text { stabilization of axin } 1 \rightarrow \\
\text { Enhancement of axin- } \\
\text { GSK3 } \beta \\
\text { complex formation } \rightarrow \\
\text { Inhibition of Wnt Signaling }\end{array}$ & $\mathrm{n} / \mathrm{a}$ & Li et al. (2015) \\
\hline SKL2001 & In vitro & 3. Inter & $\begin{array}{l}\text { Disruption of the axin/ } \\
\quad \beta \text {-catenin interaction } \rightarrow \\
\text { Activation of Wnt signaling } \\
\text { tions Targeting CK1 }\end{array}$ & $\mathrm{n} / \mathrm{a}$ & Gwak et al. (2012) \\
\hline Pyrvinium & $\begin{array}{l}\text { In vitro and in vivo } \\
\text { (Xenopus embryo } \\
\text { and mice) }\end{array}$ & CK1 & $\begin{array}{l}\text { Induction of CK } 1 \alpha \rightarrow \\
\text { Enhancement of axin- } \\
\text { GSK3 } \beta \text { complex formation } \rightarrow \\
\text { Inhibition of Wnt Signaling }\end{array}$ & $\mathrm{n} / \mathrm{a}$ & $\begin{array}{l}\text { Saraswati et al. } \\
\text { (2010), Thorne } \\
\text { et al. }(2010)\end{array}$ \\
\hline CKI-7 & $\begin{array}{l}\text { In vitro (Xenopus } \\
\text { embryos and } \\
\text { C. elegans) }\end{array}$ & CK1/DVL & $\begin{array}{l}\text { Inhibition of CK and DVL } \rightarrow \\
\text { Inhibition of Wnt Signaling }\end{array}$ & $\mathrm{n} / \mathrm{a}$ & Peters et al. (1999) \\
\hline IC261 & In vitro & CK1 & $\begin{array}{l}\text { Targeting of CK1 } 1 \varepsilon \\
\text { Inhibition of Wnt Signaling }\end{array}$ & $\begin{array}{l}\mathrm{IC}_{50}: 0.5-86 \mu \mathrm{M} \\
\quad \text { (depending on cell } \\
\text { line treated) }\end{array}$ & Kim et al. (2010b) \\
\hline SB203580 & In vitro & CK1 & $\begin{array}{l}\text { Targeting of CK1 } \delta / \varepsilon \rightarrow \\
\text { Inhibition of Wnt Signaling }\end{array}$ & $?$ & Laco et al. (2015) \\
\hline SR-3029 & $\begin{array}{l}\text { In vitro and in vivo } \\
\text { (mice) }\end{array}$ & $\begin{array}{l}\text { CK1 } \\
\text { 4. Interve }\end{array}$ & $\begin{array}{l}\text { Targeting of CK } 1 \delta \rightarrow \\
\text { Inhibition of Wnt Signaling } \\
\text { ons Activating GSK3 } \beta\end{array}$ & $\mathrm{n} / \mathrm{a}$ & $\begin{array}{l}\text { Rosenberg et al. } \\
\quad(2015)\end{array}$ \\
\hline Curcumin & In vitro & $\operatorname{GSK} 3 \beta$ & $\begin{array}{l}\text { Akt inhibits p-GSK3 } \beta \rightarrow \\
\text { activation of GSK3 } \beta \rightarrow \\
\text { Enhancement of } \\
\text { destruction complex } \overrightarrow{\text { Signaling }} \\
\text { Inhibition of Wnt Sign }\end{array}$ & $\begin{array}{c}\mathrm{IC}_{50}: 25 \mathrm{mM} \text { (after } \\
24 \text { hours treatment) } \\
\text { and } \\
18.4 \mathrm{mM} \text { (after } \\
48 \text { hours treatment) }\end{array}$ & Choi et al. (2010) \\
\hline $\begin{array}{l}\text { 9-Hydroxycanthin- } \\
\text { 6-one }\end{array}$ & $\begin{array}{l}\text { In vitro and in vivo } \\
\text { (zebrafish embryos) }\end{array}$ & GSK3 $\beta$ & $\begin{array}{l}\text { Induction of GSK } 3 \beta \rightarrow \\
\text { Inhibition of Wnt Signaling } \\
\text { (without involvement of CK) }\end{array}$ & $\begin{array}{l}\mathrm{IC}_{50}: 36.7->40 \mu \mathrm{M} \\
\quad \text { (depending on cell } \\
\text { line) }\end{array}$ & Ohishi et al. (2015) \\
\hline $\begin{array}{l}\text { All-trans retinoic } \\
\text { acid (ATRA) }\end{array}$ & In vitro & GSK3 $\beta$ & $\begin{array}{l}\text { Induction of GSK } 3 \beta \rightarrow \\
\text { Inhibition of Wnt Signaling }\end{array}$ & $\mathrm{n} / \mathrm{a}$ & Zhu et al. (2015) \\
\hline S-ibuprofen & In vitro & 5. Interve & $\begin{array}{l}\text { Induction of p-GSK } 3 \beta \rightarrow \\
\text { Inhibition of Wnt Signaling } \\
\text { (NF- } \kappa \text { B also involved) } \\
\text { ns Inhibiting GSK3 } \beta\end{array}$ & $\mathrm{n} / \mathrm{a}$ & $\begin{array}{l}\text { Greenspan et al. } \\
\quad(2011)\end{array}$ \\
\hline Lithium & In vitro & $\begin{array}{l}\text { GSK3 } \beta \\
\text { (and } \beta \text {-catenin) }\end{array}$ & $\begin{array}{l}\text { Inhibition of GSK3 } \beta(+ \text { up- } \\
\text { regulation of } \beta \text {-catenin }) \rightarrow \\
\text { Activation of Wnt Signaling }\end{array}$ & $\mathrm{n} / \mathrm{a}$ & Wexler et al. (2008) \\
\hline Valproic acid & In vitro & GSK3 $\beta$ & $\begin{array}{l}\text { Inhibition of GSK } 3 \beta \rightarrow \\
\quad \text { Activation of Wnt Signaling }\end{array}$ & $\mathrm{n} / \mathrm{a}$ & Chen et al. (1999) \\
\hline $\begin{array}{l}\text { 6-bromoindirubin- } \\
3^{\prime} \text {-oxime } \\
\text { (BIO) }\end{array}$ & $\begin{array}{l}\text { In vitro and in vivo } \\
\text { (mice) }\end{array}$ & GSK3 $\beta$ & $\begin{array}{l}\text { Competitive inhibition of } \\
\text { GSK } 3 \beta \rightarrow \text { Activation of Wnt } \\
\text { Signaling }\end{array}$ & $\mathrm{n} / \mathrm{a}$ & Kohler et al. (2014) \\
\hline
\end{tabular}


TABLE 4-Continued

\begin{tabular}{|c|c|c|c|c|c|}
\hline Intervention & Use & Proposed target & Effect & Affinity & Reference \\
\hline & $\begin{array}{l}\text { In vitro (incl. stem } \\
\text { cells) }\end{array}$ & GSK3 $\beta$ & $\begin{array}{l}\text { Inhibition of GSK3 } \beta \overrightarrow{ } \\
\quad \text { Activation of Wnt Signaling }\end{array}$ & $\mathrm{n} / \mathrm{a}$ & $\begin{array}{l}\text { Sato et al. (2004), } \\
\text { Wen et al. (2010), } \\
\text { Tseng et al. (2006) }\end{array}$ \\
\hline IBU-PO & In vitro & GSK3 $\beta$ & $\begin{array}{l}\text { Induction of p-GSK3 } \beta \rightarrow \\
\text { Inhibition of GSK3 } \beta \rightarrow \\
\text { Activation of Wnt Signaling }\end{array}$ & $\mathrm{n} / \mathrm{a}$ & Farias et al. (2005) \\
\hline Curcumin & In vitro & $\begin{array}{l}\text { GSK3 } \beta \\
\text { (and axin) }\end{array}$ & $\begin{array}{l}\text { Inhibition of GSK3 } \beta \text { (and axin) } \\
\rightarrow \text { Activation of Wnt } \\
\text { Signaling }\end{array}$ & $\mathrm{n} / \mathrm{a}$ & Ahn et al. (2010) \\
\hline $\begin{array}{l}\text { SB-216763 and } \\
\text { SB-415286 }\end{array}$ & In vitro & GSK $3 \alpha / \beta$ & $\begin{array}{l}\text { Inhibition of GSK3 } \beta \rightarrow \\
\quad \text { Activation of Wnt Signaling }\end{array}$ & $\begin{array}{l}\mathrm{ID}_{50}: 34 \mathrm{nM}(\mathrm{SB}- \\
216763) ; \mathrm{IC}_{50}: 78 \\
\text { nM (SB-415286) }\end{array}$ & Coghlan et al. (2000) \\
\hline Kenpaullone & $\begin{array}{l}\text { In vitro (stem and } \\
\text { progenitor cells) }\end{array}$ & GSK3 & $\begin{array}{l}\text { Inhibition of GSK3 } \rightarrow \\
\text { Activation of Wnt Signaling }\end{array}$ & $\mathrm{n} / \mathrm{a}$ & Lange et al. (2011) \\
\hline CHIR 99021 & $\begin{array}{l}\text { In vitro (incl. } \\
\text { embryonic stem } \\
\text { cells) }\end{array}$ & GSK3 $\alpha / \beta$ & $\begin{array}{l}\text { Inhibition of GSK3 } \beta \rightarrow \\
\quad \text { Activation of Wnt Signaling }\end{array}$ & $\mathrm{IC}_{50}: 5 \mathrm{nM}(\mathrm{GSK} 3 \beta)$ & $\begin{array}{l}\text { Bennett et al. (2002), } \\
\text { Ye et al. (2012), } \\
\text { Hou et al. (2013) } \\
\text { Naujok et al. (2014) }\end{array}$ \\
\hline CG0009 & In vitro & GSK3 $\beta$ & $\begin{array}{l}\text { Induction of Ser } 9 \text { p-GSK } 3 \beta \text { and } \\
\text { inhibition of Tyr } 215 \\
\text { p-GSK } 3 \beta \rightarrow \text { Inhibition of } \\
\text { GSK } \beta \rightarrow \text { Activation of Wnt } \\
\text { Signaling }\end{array}$ & $\begin{array}{r}\mathrm{IC}_{50}: 0.49->100 \mu \mathrm{M} \\
\text { (depending on cell } \\
\text { type in the assay) }\end{array}$ & Kim et al. (2013) \\
\hline L803-mts & $\begin{array}{l}\text { In vitro and in vivo } \\
\text { (mice) }\end{array}$ & GSK3 & $\begin{array}{l}\text { Inhibition of GSK3 } \rightarrow \\
\text { Activation of Wnt Signaling }\end{array}$ & IC50: $40 \mu \mathrm{M}$ & $\begin{array}{l}\text { Plotkin et al. (2003), } \\
\text { Kaidanovich- } \\
\text { Beilin et al. (2004) }\end{array}$ \\
\hline
\end{tabular}

misfolded $\mathrm{FZD}_{4}$ protein observed in familiar exudative vitreoretinopathy, restoring the impaired transport of this mutated receptor to the plasma membrane (Generoso et al., 2015).

Lee et al. (2015) identified five different novel and very potent molecules (1094-0205, 2124-0331, 3235-0367 and the two most potent ones, NSC36784 and NSC654259) that bind to the CRD of $\mathrm{FZD}_{8}$ and can regulate WNT signaling. All five compounds could inhibit the WNT3Amediated signaling in low micromolar concentrations. Interestingly, all five compounds showed LRP6inhibitory activity, although the authors could not exclude that the FZD CRD inhibitors might be able to target other FZD receptor's CRDs (other than $\mathrm{FZD}_{8}$ ).

Niclosamide is an anthelmintic drug, used against tapeworm infestations; nevertheless, its antitumor properties have been demonstrated in a variety of cancers. It has been shown to act via WNT signaling (Li et al., 2014c), albeit not exclusively, and the targeting appears to be dependent on the context: it caused internalization of $\mathrm{FZD}_{1}$ in an osteosarcoma cell line (U2OS) (Chen et al., 2009b) and attenuation of DVL2 in a colorectal cell line (Osada et al., 2011). Conversely, studies on prostate and breast cancer cell lines suggested that LRP6 suppression is a direct effect of treatment with niclosamide ( $\mathrm{Lu}$ et al., 2011b). These findings show it is important to make the choice of niclosamide treatment heavily dependent on the type of cancer to be treated (Osada et al., 2011). Interestingly, curcumin (which will be discussed in more depth later) was found to suppress the expression of $\mathrm{FZD}_{1}$ eightfold in MDA-1986 squamous cell carcinoma cell line, a finding that was paradoxical, because curcumin was always regarded to work more downstream (Yan et al., 2005). In this context, Zheng et al. (2017) very recently showed that curcumin might have additional inhibitory effects on WNT3A and LRP6. Previously, it was shown that WNT10B, FZD 2 , and LRP5 might be activated, leading to amplification of the cascade (Ahn et al., 2010). These findings strongly suggest that curcumin could also exhibit its effects more upstream in the pathway, affecting the binding of the WNT ligand to its receptor (FZD) and to its coreceptor (LRP). In addition, there is evidence stressing potential deleterious effects of curcumin (even promoting cancer in some cases), thus more research is urgently needed to ascertain the toxicologic profile of the compound and its benefit-to-risk ratio (Burgos-Moron et al., 2010).

Sclerostin is an endogenous antagonist of the WNT cascade and is expressed practically exclusively by osteocytes. Sclerostin binds LRP5/6 (Li et al., 2005) and disrupts the LRP5/6-FZD complex formation. In fact, it binds more strongly to LRP6, whereas antisclerostin antibodies can reverse the sclerostin-mediated LRP6 inhibition (van Dinther et al., 2013) and prevent noxious effects on the bones (Tian et al., 2011).

Because phosphorylation of LRP6 is an essential element of WNT-mediated activation, interaction with the LRP5/6 coreceptor is another major point where pharmacological interventions could act on to influence the signaling cascade. Salinomycin is a potassium ionophore compound that was shown to possess WNT antagonistic activity. Salinomycin was found to inhibit the $\mathrm{WNT} 1 / \mathrm{FZD}_{5} / \mathrm{LRP} 6$ and $\mathrm{WNT} 3 / \mathrm{FZD}_{5} / \mathrm{LRP} 6$ complexes, to robustly reduce LRP6 protein levels, and to induce degradation of LRP6 in chronic lymphocytic 
TABLE 5

Interventions in $\beta$-catenin and gene transcription

\begin{tabular}{|c|c|c|c|c|c|}
\hline Intervention & Use & Proposed target & Effect & Affinity & Reference \\
\hline \multicolumn{6}{|c|}{ 1. Targeting of the TCF/LEF Transcription Factors } \\
\hline $\begin{array}{l}\text { iCRT3, iCRT5 and } \\
\text { iCRT14 }\end{array}$ & In vitro & $\begin{array}{l}\beta \text {-catenin- } \\
\text { TCF/LEF } \\
\text { complex }\end{array}$ & $\begin{array}{l}\text { Interference with the } \\
\beta \text {-catenin/TCF } \\
\text { interaction } \rightarrow \text { Inhibition } \\
\text { of WNT Signaling }\end{array}$ & $\begin{array}{l}\text { IC }_{50}: 8.2 \mathrm{nM}(\mathrm{iCRT} 3) ; \\
\mathrm{IC}_{50}: 18.7 \mathrm{nM} \\
\text { (iCRT5); IC } \mathrm{IO}_{50}: \\
\text { 40.3 nM (iCRT14) - in } \\
\text { STF16 luciferase } \\
\text { assay }\end{array}$ & $\begin{array}{l}\text { Gonsalves et al. } \\
\text { (2011), } \\
\text { Griffiths et al. } \\
(2015)\end{array}$ \\
\hline Thiazole and oxazole & In vitro & $\begin{array}{l}\beta \text {-catenin- } \\
\text { TCF/LEF } \\
\text { complex }\end{array}$ & $\begin{array}{l}\text { Interference with the } \\
\beta \text {-catenin/TCF } 4 \\
\text { interaction } \rightarrow \text { Inhibition } \\
\text { of WNT Signaling }\end{array}$ & $\mathrm{n} / \mathrm{a}$ & $\begin{array}{l}\text { Narayanan et al. } \\
(2012)\end{array}$ \\
\hline $\begin{array}{l}\text { 15-oxospiramilactone } \\
\text { (NC043) }\end{array}$ & In vitro & $\begin{array}{l}\beta \text {-catenin- } \\
\text { TCF/LEF } \\
\text { complex }\end{array}$ & $\begin{array}{l}\text { Interference with the } \\
\beta \text {-catenin/TCF } 4 \\
\text { interaction } \rightarrow \text { Inhibition } \\
\text { of WNT Signaling }\end{array}$ & $\begin{array}{l}\mathrm{IC}_{50}: 0.9-6.9 \mu \mathrm{M} \\
\text { (depending on the cell } \\
\text { type) }\end{array}$ & $\begin{array}{l}\text { Wang et al. } \\
\text { (2011b) }\end{array}$ \\
\hline Henryin & In vitro & $\begin{array}{l}\beta \text {-catenin- } \\
\text { TCF/LEF } \\
\text { complex }\end{array}$ & $\begin{array}{l}\text { Interference with the } \\
\beta \text {-catenin/TCF } 4 \\
\text { interaction } \rightarrow \text { Inhibition } \\
\text { of WNT Signaling }\end{array}$ & $\begin{array}{l}\mathrm{IC}_{50}: 0.6 \pm 0.02 \mu \mathrm{M}(\mathrm{ST}- \\
\quad \text { luciferase assay) }\end{array}$ & Li et al. (2013c) \\
\hline $\begin{array}{l}11 \alpha, 12 \alpha- \\
\quad \text { epoxyleukamenin } \mathrm{E} \\
\quad(\mathrm{EPLE})\end{array}$ & In vitro & $\begin{array}{l}\beta \text {-catenin- } \\
\text { TCF/LEF } \\
\text { complex }\end{array}$ & $\begin{array}{l}\text { Interference with the } \\
\beta \text {-catenin/TCF } 4 \\
\text { interaction } \rightarrow \text { Inhibition } \\
\text { of WNT Signaling }\end{array}$ & $\mathrm{n} / \mathrm{a}$ & Ye et al. (2015) \\
\hline LF3 & $\begin{array}{l}\text { In silico, in vitro and } \\
\text { in vivo (mice) }\end{array}$ & $\begin{array}{l}\beta \text {-catenin- } \\
\text { TCF/LEF } \\
\text { complex }\end{array}$ & $\begin{array}{l}\text { Interference with the } \\
\beta \text {-catenin/TCF } 4 \\
\text { interaction } \rightarrow \text { Inhibition } \\
\text { of WNT Signaling }\end{array}$ & $\begin{array}{c}\mathrm{IC}_{50}: 2.4-4.0 \mathrm{mM}(\mathrm{HeLa} \\
\text { cells); } \mathrm{IC}_{50}: 22.2 \pm \\
4.9 \mathrm{mM}(\mathrm{HEK} 293 \\
\text { TOPFlash assay) }\end{array}$ & Fang et al. (2016) \\
\hline BC-21 & In silico and in vitro & $\begin{array}{l}\beta \text {-catenin- } \\
\text { TCF/LEF } \\
\text { complex }\end{array}$ & $\begin{array}{l}\text { Interference with the } \\
\beta \text {-catenin/TCF } 4 \\
\text { interaction } \rightarrow \text { Inhibition } \\
\text { of WNT Signaling }\end{array}$ & $\begin{array}{l}\mathrm{IC}_{50}: 5 \mu \mathrm{M}(\beta \text {-catenin- } \\
\text { TCF4 inhibition } \\
\text { assay) }\end{array}$ & Tian et al. (2012) \\
\hline $\begin{array}{l}\text { BC-23 (monotherapy or } \\
\text { with radiation } \\
\text { therapy) }\end{array}$ & In silico and in vitro & $\begin{array}{l}\beta \text {-catenin- } \\
\text { TCF/LEF } \\
\text { complex }\end{array}$ & $\begin{array}{l}\text { Interference with the } \\
\beta \text {-catenin/TCF } 4 \\
\text { interaction } \rightarrow \text { Inhibition } \\
\text { of WNT Signaling }\end{array}$ & $\begin{array}{l}\mathrm{IC}_{50}: 5 \mu \mathrm{M}(\beta \text {-catenin- } \\
\text { TCF4 inhibition } \\
\text { assay) }\end{array}$ & $\begin{array}{l}\text { Zhang et al. } \\
\qquad(2016 a)\end{array}$ \\
\hline PNU-74654 & In vitro & $\begin{array}{l}\beta \text {-catenin- } \\
\text { TCF/LEF } \\
\text { complex }\end{array}$ & $\begin{array}{l}\text { Interference with the } \\
\beta \text {-catenin/TCF } \\
\text { interaction } \rightarrow \text { Inhibition } \\
\text { of WNT Signaling }\end{array}$ & $\begin{array}{l}\mathrm{IC}_{50}: 117.2-5026.0 \mu \mathrm{M} \\
\text { (depending on time } \\
\text { and cell line used in } \\
\text { the assay) }\end{array}$ & Leal et al. (2015) \\
\hline PKF115-584 & $\begin{array}{l}\text { In vitro and in vivo } \\
\text { (Xenopus embryos) } \\
\text { In vitro and in vivo } \\
\text { (mice) }\end{array}$ & $\begin{array}{l}\beta \text {-catenin- } \\
\text { TCF/LEF } \\
\text { complex }\end{array}$ & $\begin{array}{l}\text { Interference with the } \\
\beta \text {-catenin/TCF4 } \\
\text { interaction, TCF binding } \\
\text { with DNA, } \beta \text {-catenin/ } \\
\text { APC complex formation } \\
\text { and antagonism of } \\
\text { exogenous } \beta \text {-catenin } \rightarrow \\
\text { Inhibition of WNT } \\
\text { Signaling }\end{array}$ & $\begin{array}{l}\mathrm{IC}_{50}: 3.2 \mu \mathrm{M}(\beta \text {-catenin - } \\
\text { TCF4 inhibition } \\
\text { assay) } \\
\mathrm{IC}_{50}: 0.31-2.05 \mu \mathrm{M} \\
\text { (sphere forming } \\
\text { assay, range } \\
\text { depending on cell } \\
\text { type) }\end{array}$ & $\begin{array}{l}\text { Lepourcelet et al. } \\
\text { (2004), Hallett } \\
\text { et al. (2012) }\end{array}$ \\
\hline CGP049090 & $\begin{array}{l}\text { In vitro and in vivo } \\
\text { (Xenopus embryos) }\end{array}$ & $\begin{array}{l}\beta \text {-catenin- } \\
\text { TCF/LEF } \\
\text { complex }\end{array}$ & $\begin{array}{l}\text { Interference with the } \\
\beta \text {-catenin/TCF4 } \\
\text { interaction, TCF binding } \\
\text { with DNA, } \beta \text {-catenin/ } \\
\text { APC complex formation } \\
\text { and antagonism of } \\
\text { exogenous } \beta \text {-catenin } \rightarrow \\
\text { Inhibition of WNT } \\
\text { Signaling }\end{array}$ & $\begin{array}{l}\mathrm{IC}_{50}: 8.7 \mu \mathrm{M}(\beta \text {-catenin- } \\
\text { TCF4 inhibition } \\
\text { assay) }\end{array}$ & $\begin{array}{l}\text { Lepourcelet et al. } \\
\text { (2004) }\end{array}$ \\
\hline PKF222-815 & $\begin{array}{l}\text { In vitro and in vivo } \\
\text { (Xenopus embryos) }\end{array}$ & $\begin{array}{l}\beta \text {-catenin- } \\
\text { TCF/LEF } \\
\text { complex }\end{array}$ & $\begin{array}{l}\text { Interference with the } \\
\beta \text {-catenin/TCF4 } \\
\text { interaction and } \\
\text { antagonism of exogenous } \\
\beta \text {-catenin } \rightarrow \text { Inhibition of } \\
\text { WNT Signaling }\end{array}$ & $\begin{array}{l}\mathrm{IC}_{50}: 4.1 \mu \mathrm{M}(\beta \text {-catenin - } \\
\text { TCF4 inhibition } \\
\text { assay) }\end{array}$ & $\begin{array}{l}\text { Lepourcelet et al. } \\
\text { (2004) }\end{array}$ \\
\hline \multirow[t]{2}{*}{ PKF118-310 } & $\begin{array}{l}\text { In vitro and in vivo } \\
\text { (mice) }\end{array}$ & $\begin{array}{l}\beta \text {-catenin- } \\
\text { TCF/LEF } \\
\text { complex }\end{array}$ & $\begin{array}{l}\text { Interference with the } \\
\beta \text {-catenin/TCF } 4 \\
\text { interaction and } \\
\text { antagonism of exogenous } \\
\beta \text {-catenin } \rightarrow \text { Inhibition of } \\
\text { WNT Signaling }\end{array}$ & $\begin{array}{l}\mathrm{IC}_{50}: 0.8 \mu \mathrm{M}(\beta \text {-catenin- } \\
\text { TCF4 inhibition } \\
\text { assay })\end{array}$ & $\begin{array}{l}\text { Lepourcelet et al. } \\
\text { (2004) }\end{array}$ \\
\hline & $\begin{array}{l}\text { In vitro and in vivo } \\
\text { (mice) }\end{array}$ & & & $\begin{array}{l}\mathrm{IC}_{50}: 0.54-1.54 \mu \mathrm{M} \\
\quad \text { (sphere forming } \\
\text { assay, range } \\
\text { depending on cell } \\
\text { type) }\end{array}$ & $\begin{array}{l}\text { Hallett et al. } \\
\quad(2012)\end{array}$ \\
\hline
\end{tabular}


TABLE 5 -Continued

\begin{tabular}{|c|c|c|c|c|c|}
\hline Intervention & Use & Proposed target & Effect & Affinity & Reference \\
\hline $\operatorname{Daxx}$ & In vitro & $\begin{array}{l}\beta \text {-catenin- } \\
\text { TCF/LEF } \\
\text { complex }\end{array}$ & $\begin{array}{l}\text { Binding to TCF } 4 \rightarrow \\
\text { Potentiation of } \beta \text {-catenin/ } \\
\text { TCF4-mediated } \\
\text { transcriptional activation } \\
\rightarrow \text { Activation of WNT } \\
\text { Signaling }\end{array}$ & $\mathrm{n} / \mathrm{a}$ & $\begin{array}{l}\text { Huang and Shih } \\
\quad(2009)\end{array}$ \\
\hline Quercetin & In vitro & $\begin{array}{l}\beta \text {-catenin- } \\
\text { TCF/LEF } \\
\text { complex }\end{array}$ & $\begin{array}{l}\text { Interference with the } \\
\beta \text {-catenin/TCF4 } \\
\text { interaction } \rightarrow \text { Inhibition } \\
\text { of WNT Signaling }\end{array}$ & $\mathrm{n} / \mathrm{a}$ & Park et al. (2005) \\
\hline Isoquercitrin & $\begin{array}{l}\text { In vitro and in vivo } \\
\text { (Xenopus embryos) }\end{array}$ & $\begin{array}{l}\beta \text {-catenin- } \\
\text { TCF/LEF } \\
\text { complex }\end{array}$ & $\begin{array}{l}\text { Interference with the } \\
\beta \text {-catenin/TCF } \\
\text { interaction } \rightarrow \text { Inhibition } \\
\text { of WNT Signaling }\end{array}$ & $\mathrm{n} / \mathrm{a}$ & $\begin{array}{l}\text { Amado et al. } \\
\text { (2014) }\end{array}$ \\
\hline $\begin{array}{l}\text { 6,7-dihydroxycoumarin } \\
\text { (Aesculetin) }\end{array}$ & $\begin{array}{l}\text { In vitro and in vivo } \\
\text { (Xenopus embryos } \\
\text { and mice) }\end{array}$ & $\begin{array}{l}\beta \text {-catenin- } \\
\text { TCF/LEF } \\
\text { complex }\end{array}$ & $\begin{array}{l}\text { Interference with the } \\
\beta \text {-catenin/TCF } 4 \\
\text { interaction } \rightarrow \text { Inhibition } \\
\text { of WNT Signaling }\end{array}$ & $\mathrm{n} / \mathrm{a}$ & Lee et al. (2013) \\
\hline \multirow[t]{2}{*}{ Curcumin } & In vitro & $\begin{array}{l}\beta \text {-catenin- } \\
\text { TCF/LEF } \\
\text { complex }\end{array}$ & $\begin{array}{l}\text { Interference with the } \\
\beta \text {-catenin/TCF4 } \\
\text { interaction } \rightarrow \text { Inhibition } \\
\text { of WNT Signaling }\end{array}$ & $\mathrm{n} / \mathrm{a}$ & $\begin{array}{l}\text { Jaiswal et al., } \\
2002\end{array}$ \\
\hline & In vitro & $\begin{array}{l}\beta \text {-catenin- } \\
\text { TCF/LEF } \\
\text { complex }\end{array}$ & $\begin{array}{l}\text { Decrease of TCF4, CBP and } \\
\text { p300 protein levels } \rightarrow \\
\text { Interference with the } \\
\beta \text {-catenin/TCF4 } \\
\text { interaction } \rightarrow \text { Inhibition } \\
\text { of WNT Signaling }\end{array}$ & $\mathrm{n} / \mathrm{a}$ & $\begin{array}{l}\text { Teiten et al. } \\
\quad(2011)\end{array}$ \\
\hline Aspirin & In vitro & $\begin{array}{l}\beta \text {-catenin- } \\
\text { TCF gene } \\
\text { transcription }\end{array}$ & $\begin{array}{l}\text { Suppression of gene } \\
\text { transcription of } \\
\beta \text {-catenin/TCF } 4 \rightarrow \\
\text { Inhibition of WNT } \\
\text { Signaling }\end{array}$ & $\mathrm{n} / \mathrm{a}$ & $\begin{array}{l}\text { Dihlmann et al. } \\
\text { (2001) }\end{array}$ \\
\hline Indomethacin & In vitro & $\begin{array}{l}\beta \text {-catenin- } \\
\text { TCF gene } \\
\text { transcription }\end{array}$ & $\begin{array}{l}\text { Suppression of gene } \\
\text { transcription of } \\
\beta \text {-catenin/TCF } 4 \rightarrow \\
\text { Inhibition of WNT } \\
\text { Signaling }\end{array}$ & $\mathrm{n} / \mathrm{a}$ & $\begin{array}{l}\text { Dihlmann et al. } \\
\text { (2001) }\end{array}$ \\
\hline $\begin{array}{l}\text { Celecoxib and 2,5- } \\
\text { dimethyl-celecoxib }\end{array}$ & In vivo (mice) & $\begin{array}{l}\beta \text {-catenin and } \\
\text { TCF4 } \\
\text { expression }\end{array}$ & $\begin{array}{l}\text { Suppression of } \beta \text {-catenin } \\
\text { and TCF } 4 \text { expression (not } \\
\text { clear if the complex } \\
\text { formation itself is } \\
\text { affected) } \rightarrow \text { Inhibition of } \\
\text { TCF-mediated } \\
\text { transcription } \rightarrow \\
\text { Inhibition of WNT } \\
\text { Signaling }\end{array}$ & $\mathrm{n} / \mathrm{a}$ & $\begin{array}{l}\text { Fan et al. (2011), } \\
\text { Egashira et al. } \\
(2017)\end{array}$ \\
\hline \multirow[t]{2}{*}{ Sulindac } & $\begin{array}{l}\text { In vitro and in vivo } \\
\text { (mice) }\end{array}$ & $\begin{array}{l}\text { TCF gene } \\
\text { transcription }\end{array}$ & $\begin{array}{l}\text { Suppression of gene } \\
\text { transcription of TCF } \rightarrow \\
\text { Inhibition of WNT } \\
\text { Signaling }\end{array}$ & $\begin{array}{l}\mathrm{IC}_{50}: 1.8-33.9 \mu \mathrm{M} \text { (for } \\
\text { COX-1/2 and cGMP) }\end{array}$ & $\begin{array}{l}\text { Tinsley et al. } \\
\text { (2010) }\end{array}$ \\
\hline & In vitro & $\begin{array}{l}\beta \text {-catenin and } \\
\text { TCF } 4 \\
\text { expression }\end{array}$ & $\begin{array}{l}\text { Suppression of gene } \\
\text { transcription of TCF and } \\
\beta \text {-catenin synthesis } \rightarrow \\
\text { Inhibition of WNT } \\
\text { Signaling }\end{array}$ & $\begin{array}{l}\mathrm{IC}_{50}: 75-83 \mu \mathrm{M} \\
\quad \text { (following } 72 \text { hour } \\
\text { treatment of various } \\
\text { human cancer cell } \\
\text { lines) }\end{array}$ & Li et al. (2013) \\
\hline \multirow[t]{2}{*}{ ICAT } & $\begin{array}{l}\text { In vitro and in vivo } \\
\text { (Xenopus embryos) }\end{array}$ & $\begin{array}{l}\beta \text {-catenin- } \\
\text { TCF/LEF } \\
\text { complex }\end{array}$ & $\begin{array}{l}\text { Interference with the } \\
\beta \text {-catenin/TCF } 4 \\
\text { interaction } \rightarrow \text { Inhibition } \\
\text { of WNT Signaling }\end{array}$ & $\mathrm{n} / \mathrm{a}$ & Tago et al. (2000) \\
\hline & In vitro & $\begin{array}{l}\beta \text {-catenin- } \\
\text { TCF/LEF } \\
\text { complex }\end{array}$ & $\begin{array}{l}\text { Interference with the } \\
\beta \text {-catenin/TCF/LEF } \\
\text { interaction } \rightarrow \text { Inhibition } \\
\text { of WNT Signaling }\end{array}$ & $\mathrm{n} / \mathrm{a}$ & $\begin{array}{l}\text { Daniels and Weis } \\
\quad(2002)\end{array}$ \\
\hline ICG-001 (PRI-724) & $\begin{array}{l}\text { In vitro, in vivo (mice) } \\
\text { and } \mathrm{CT}\end{array}$ & $\mathrm{CBP}$ & $\begin{array}{l}\text { ting of } C B P \\
\text { Disruption of the } \beta \text {-catenin/ } \\
\text { CBP interaction } \rightarrow \\
\text { Inhibition of WNT } \\
\text { Signaling }\end{array}$ & $\begin{array}{l}\mathrm{IC}_{50}: 3 \mu \mathrm{M} \text { (TOPFlash } \\
\quad \text { assay) }\end{array}$ & $\begin{array}{l}\text { Emami et al. } \\
\text { (2004), } \\
\text { Henderson } \\
\text { et al. (2010), } \\
\text { El-Khoueiry } \\
\text { et al. (2013), } \\
\text { Sasaki et al. } \\
\text { (2013) }\end{array}$ \\
\hline PMED-1 & In silico and in vitro & CBP & $\begin{array}{l}\text { Disruption of the } \beta \text {-catenin/ } \\
\text { CBP interaction } \rightarrow \\
\text { Inhibition of WNT } \\
\text { Signaling }\end{array}$ & $\begin{array}{l}\mathrm{IC}_{50}: 4.87-32 \mu \mathrm{M} \\
\text { (depending on cell } \\
\text { type in the TOPFlash } \\
\text { assay) }\end{array}$ & $\begin{array}{l}\text { Delgado et al. } \\
\quad(2014)\end{array}$ \\
\hline
\end{tabular}


TABLE 5 -Continued

\begin{tabular}{|c|c|c|c|c|c|}
\hline Intervention & Use & Proposed target & Effect & Affinity & Reference \\
\hline Curcumin & In vitro & 3. Targ & $\begin{array}{l}\text { Decrease of TCF4, CBP and } \\
\text { p300 protein levels } \rightarrow \\
\text { Interference with the } \\
\beta \text {-catenin/TCF4 } \\
\text { interaction } \rightarrow \text { Inhibition } \\
\text { of WNT Signaling } \\
\text { ting of p300 }\end{array}$ & $\mathrm{n} / \mathrm{a}$ & $\begin{array}{l}\text { Teiten et al. } \\
\text { (2011) }\end{array}$ \\
\hline ICAT & In vitro & p300 & $\begin{array}{l}\text { Inhibition of the } \beta \text {-catenin/ } \\
\text { Lef1/p300 interaction } \rightarrow \\
\text { Inhibition of WNT } \\
\text { Signaling }\end{array}$ & $\mathrm{n} / \mathrm{a}$ & $\begin{array}{l}\text { Daniels and Weis } \\
\quad(2002)\end{array}$ \\
\hline $\begin{array}{l}\text { ICAT- } 61 \text { (helical domain } \\
\text { of ICAT) }\end{array}$ & In vitro & p300 & $\begin{array}{l}\text { Disruption of the } \beta \text {-catenin/ } \\
\text { p300 interaction (but no } \\
\text { interference with the } \\
\text { binding of TCF/LEF) } \rightarrow \\
\text { Inhibition of WNT } \\
\text { Signaling }\end{array}$ & $\mathrm{n} / \mathrm{a}$ & $\begin{array}{l}\text { Daniels and Weis } \\
\quad(2002)\end{array}$ \\
\hline Windorphen (WD) & $\begin{array}{l}\text { In vitro and in vivo } \\
\text { (Zebrafish embryos) }\end{array}$ & p300 & $\begin{array}{l}\text { Disruption of the } \beta \text {-catenin/ } \\
\text { p300 interaction } \rightarrow \\
\text { Inhibition of WNT } \\
\text { Signaling }\end{array}$ & $\begin{array}{l}\mathrm{IC}_{50}: 4.2 \mu \mathrm{M}(\mathrm{p} 300) ; \\
\mathrm{IC}_{50}: 1.5 \mu \mathrm{M}(\mathrm{WNT} 3 \mathrm{a}- \\
\text { inducible TOPFlash } \\
\text { assay) }\end{array}$ & Hao et al. (2013) \\
\hline IQ1 & $\begin{array}{l}\text { In vitro and in vivo } \\
\text { (mice) }\end{array}$ & p300 & $\begin{array}{l}\text { Disruption of the } \beta \text {-catenin/ } \\
\text { p300 interaction } \rightarrow \\
\text { Amplification of the } \\
\beta \text {-catenin/TCF4 } \\
\text { interaction } \rightarrow \text { Activation } \\
\text { of WNT Signaling } \\
\text { (paradox?) }\end{array}$ & $\mathrm{n} / \mathrm{a}$ & $\begin{array}{l}\text { Sasaki and Kahn } \\
\quad(2014)\end{array}$ \\
\hline $\begin{array}{l}\text { Bisdemethoxycurcumin } \\
\text { (BDMC) and } \\
\text { demethoxycurcumin } \\
\text { (DMC) }\end{array}$ & In vitro & p300 & $\begin{array}{l}\text { Downregulation of p300 } \\
\quad \text { (without effects on TCF4 } \\
\text { or } \beta \text {-catenin levels) } \rightarrow \\
\text { Inhibition of WNT } \\
\text { Signaling }\end{array}$ & $\mathrm{n} / \mathrm{a}$ & Ryu et al. (2008) \\
\hline Curcumin & In vitro & 4. Targ & $\begin{array}{l}\text { Decrease of TCF4, CBP and } \\
\text { p300 protein levels } \rightarrow \\
\text { Interference with the } \\
\beta \text {-catenin/TCF4 } \\
\text { interaction } \rightarrow \text { Inhibition } \\
\text { of WNT Signaling } \\
\text { ing of BCL9 }\end{array}$ & $\mathrm{n} / \mathrm{a}$ & $\begin{array}{l}\text { Teiten et al. } \\
\text { (2011) }\end{array}$ \\
\hline \multirow[t]{2}{*}{ Carnosic acid (CA) } & In silico and in vitro & $\begin{array}{l}\beta \text {-catenin/BCL9 } \\
\text { complex }\end{array}$ & $\begin{array}{l}\text { Interference with } \beta \text {-catenin/ } \\
\text { BCL9 interaction and } \\
\text { degradation of } \beta \text {-catenin } \\
\rightarrow \text { Inhibition of WNT } \\
\text { Signaling }\end{array}$ & $\mathrm{n} / \mathrm{a}$ & $\begin{array}{l}\text { de la Roche et al. } \\
\text { (2012) }\end{array}$ \\
\hline & In silico and in vitro & $\begin{array}{l}\beta \text {-catenin/BCL9 } \\
\text { complex }\end{array}$ & $\begin{array}{l}\text { Interference with } \beta \text {-catenin/ } \\
\text { BCL9 interaction } \rightarrow \\
\text { Inhibition of WNT } \\
\text { Signaling }\end{array}$ & $\begin{array}{l}\mathrm{IC}_{50}: 8.7 \pm 1.5-20 \pm \\
0.18 \mu \mathrm{M} \text { (depending } \\
\quad \text { on cell line) }\end{array}$ & $\begin{array}{l}\text { Hoggard et al. } \\
\text { (2015) }\end{array}$ \\
\hline $\begin{array}{l}\text { LATS Large Tumor } \\
\text { Suppressor (LATS) } 2\end{array}$ & $\begin{array}{l}\text { In vitro and in vivo } \\
\text { (mice) }\end{array}$ & $\begin{array}{l}\beta \text {-catenin/BCL9 } \\
\text { complex }\end{array}$ & $\begin{array}{l}\text { Interference with } \beta \text {-catenin/ } \\
\text { BCL9 interaction } \rightarrow \\
\text { Inhibition of WNT } \\
\text { Signaling }\end{array}$ & $\mathrm{n} / \mathrm{a}$ & Li et al. (2013) \\
\hline Compound 11 & In silico and in vitro & $\begin{array}{l}\beta \text {-catenin/BCL9 } \\
\text { complex }\end{array}$ & $\begin{array}{l}\text { Interference with } \beta \text {-catenin/ } \\
\text { BCL9 interaction } \rightarrow \\
\text { Inhibition of WNT } \\
\text { Signaling }\end{array}$ & $\begin{array}{l}\mathrm{IC}_{50}: 22 \pm 4.0-26 \pm \\
\quad 6.6 \mu \mathrm{M}(\text { depending on } \\
\text { cell line) }\end{array}$ & $\begin{array}{l}\text { Wisniewski et al. } \\
\text { (2016) }\end{array}$ \\
\hline SAH-BCL9 & $\begin{array}{l}\text { In vitro and in vivo } \\
\text { (mice) }\end{array}$ & $\begin{array}{l}\beta \text {-catenin/BCL9 } \\
\text { complex }\end{array}$ & $\begin{array}{l}\text { Interference with } \beta \text {-catenin/ } \\
\text { BCL9 interaction } \rightarrow \\
\text { Inhibition of WNT } \\
\text { Signaling }\end{array}$ & $\begin{array}{l}\mathrm{IC}_{50}: 135-810 \mathrm{nM} \\
\quad \text { (depending on assay) }\end{array}$ & $\begin{array}{l}\text { Takada et al. } \\
\text { (2012) }\end{array}$ \\
\hline
\end{tabular}

leukemia cells (Lu et al., 2011a). Similar results were demonstrated by $\mathrm{Lu}$ and $\mathrm{Li}$ (2014), which also implicated mTORC1 and a activation of GSK3 $\beta$ in an LRP6-mediated cross-talking mechanism, leading to antitumor effects in prostate and breast cancer.

Leptin has been shown to possess WNT signalingmediating capabilities. This weight-regulating hormone could induce the phosphorylation of LRP6 in Leptin KO mice (which show an attenuated LRP6 expression), whereas DKK1 had the opposite effect. Furthermore, leptin was shown to affect GSK3 $\beta$ levels as well as axin2 expression, implying that it possesses an activator role in the signaling (Benzler et al., 2013). 
Silibinin, is a naturally occurring compound that exhibits in vitro and in vivo antitumor effects. Silibinin attenuated the endogenous LRP6 expression at the transcription level and blocks its phosphorylation in a variety of prostate and breast cancer cell lines (Lu et al., 2012). Some research groups have provided evidence for the potential of anti-LRP6 antibodies as blockers (Ettenberg et al., 2010; Gong et al., 2010) or inducers (Gong et al., 2010) of WNT binding via LRP6-mediated mechanisms. Lastly, a 28 -amino acid peptide mimicking the LRP5/6 domain was described to interfere with the LRP5/6 and N-cadherin complex in osteoblasts. The peptide led to an activation of WNT signaling and its effects were dependent on N-cadherin (Hay et al., 2012).

The utilization of endogenous mediators of the WNT signaling pathway is also another means of interfering with the cascade. DKK1 is a natural inhibitor of the cascade (Mao et al., 2001), whereas sFRP isoforms demonstrate variable activities (see section IV.2) (Shi et al., 2007). DKK1 and DKK2 bind LRP6 at domains different to the ones required for the WNT/FZD complex formation (Mao et al., 2001). Neutralizing the effects of DKK1 by a polyclonal antibody was shown to inhibit tumor cell growth and cell invasion in vitro; the treatment was found to be relative safe (Sato et al., 2010b). A humanized monoclonal antibody against DKK1 called BHQ880 had beneficial effects in a mouse myeloma model, where it exhibited not only antitumor effects but also promoted bone formation (Fulciniti et al., 2009). Thus far, BHQ880 has been investigated in three clinical trials in myeloma patients (https:// clinicaltrials.gov./ct2/results?term=BHQ880+\&Search=Search). The trials have been completed; however, the drug is not yet available. It was reported that patients have been tolerating the treatment and that the antibody has caused meaningful improvements in the quality of their lives (Iyer et al., 2014).

Furthermore, active immunization with a DKK1 vaccine showed impressive results in boosting of T-cell production and protecting mice from myeloma or treating mice that were suffering from a myeloma tumor. The beneficial effects of the active vaccine could be further improved by the addition of B7H1-blocking or OX40agonist mAbs.

SFRP manipulations can also regulate WNT signaling. Iminooxothiazolidines (compound 1-34) are reported to be potential inhibitors of SFRP1 and to promote osteogenesis (Shi et al., 2009). Following a detailed screening of approximately 440,000 compounds, Bodine et al. (2009) also described an inhibitor of sFRP1 called WAY-316606 that led to increased bone formation; the same group proposed the use of several other similar inhibitory compounds (Moore et al., 2010).

\section{Intracellular Compartment}

1. Interventions Targeting Dishevelled. The first intracellular step following the successful binding of
WNT and FZD is the activation of the DVL proteins, which acts downstream and phosphorylates the intracellular domain of LRP5/6. The PDZ domain of DVL is of paramount importance for the transduction of the signal, because it was shown to interact directly with seventh transmembrane helix of FZD (Wong et al., 2003). Compound 3289-8625 could suppress in vitro luciferase activity by targeting the PDZ domain of DVL when the system is activated by WNT3A. Compound 3289-8625 can readily penetrate membranes (in vitro and of Xenopus) and showed a suppressive effect on $\beta$-catenin levels in prostate cancer cells (PC-3), which led to an attenuation of the cells' proliferation (Grandy et al., 2009). In addition, the organic compound NSC668036 was identified within the National Cancer Institute's small-molecular library to functionally interact with PDZ. NSC668036 was proven to counteract WNT signaling with the additional advantages of high solubility, stability, and simplicity of its molecule, making it a great candidate to produce analogs from with improved properties (Shan et al., 2005). Shan et al. (2012) also developed several compounds, with the most potent one being J01-017a; this compound demonstrated improved binding affinity for the PDZ. Additionally, another organic compound known by the short name FJ9 was proposed as a PDZ inhibitor and an antitumor molecule (Fujii et al., 2007). However, it was shown to be toxic at concentrations normally employed to inhibit WNT signaling (Zhang et al., 2009b), limiting its use. CXXC-type zinc finger protein 5 (CXXC5) was shown to interact with the PDZ domain of DVL and thus operate as a negative feedback mechanism of the WNT signaling pathway. Small inhibitors that can block the interaction between DVL and CXXC5 led to an activation of WNT signaling; Kim et al. propose three inhibitors, the peptide PolyR-DBM (Kim et al., 2015), KY-02372, and KY-02061 (Kim et al., 2016), which could be regarded as candidates of orally available antiosteoporosis therapy. Lastly, a brief communication by Zhang et al. (2009b) reported a PDZ peptide called pen-N3.

2. Interventions Targeting Axin. The scaffolding protein axin is, as previously shown, the rate-limiting factor of the destruction complex and it is regulated by tankyrases (TNK). TNKs are a class of nuclear proteins that belong to the big family of Poly (ADP-ribose) polymerases (PARP). TNKs play instrumental roles in the organization of telomere length, the regulation of the spindle assembly checkpoint, as well as the control of glucose uptake in the presence of insulin. Furthermore, it was found that the TNKs may exert regulatory control over WNT signaling by controlling the destruction of $\beta$-catenin and appear to confer inhibitory effects on several tumor types by reducing telomere length (Lehtio et al., 2013). Intriguingly, TNK inhibitors are active even in the absence of APC, thus they can be of great interest in APC mutation-related diseases that 
are accompanied by hyperactivated WNT signaling pathway (Huang et al., 2009; Waaler et al., 2012). Several groups have attempted to investigate the role of TNKs in the regulation of WNT signaling. Huang et al. (2009) published a seminal paper demonstrating for the first time that a small molecule named XAV939 can have profound effects on WNT signaling. XAV939 induced the activity of the destruction complex and thus led to the phosphorylation of $\beta$-catenin, which renders the latter inactive. Axin2 ubiquitination was suppressed and axin $1 / 2$ (protein but not mRNA) levels were substantially induced, mediating a robust increase in the axin/GSK3 $\beta$ complex formation that phosphorylates $\beta$-catenin leading to its degradation. Interestingly, XAV939 was shown to suppress proliferation and myofibroblast transdifferentiation, while inducing the differentiation of bone marrow-derived mesenchymal stem cells (Wang et al., 2014a). Lastly, the cardiovascular potential of XAV939 (as well as IWRs and IWPs) is high, taking reports into account showing important effects of the compound on promotion of cardiogenesis in human embryonic stem cells (Willems et al., 2011) and human induced pluripotent stem cells (Kadari et al., 2015). Moreover, the inhibitors of WNT response (IWRs) are also important tools in this class. Chen et al. (2009a) propose two small compounds named IWR- 1 and IWR- 2 that promote $\beta$-catenin degradation. The authors showed that this is achieved by promoting stabilization of axin, and not via changes in GSK3 $\beta$ or APC, $\beta$-catenin/axin- 2 binding affinity, or the interaction of axin with other WNT signaling components. Inhibition of the WNT cascade in keloid-derived skin fibroblasts by IWR-1 led to changes in the expression levels of MMPs (MMP-1, MMP-3, and MMP-13) (Zhou et al., 2017), this going hand-in-hand with the aforementioned findings of Wang et al. (2014a) on lung myofibroblasts.

Combined treatment with several agents targeting WNT signaling is gaining more and more interest. XAV939 can have profound effects on tumor resistance, for example in colorectal cancer (Wu et al., 2016b) in combinational regiments with chemotherapy (e.g., alongside 5-fluorouracil and cisplatin). Quite recently, Ono et al. (2014) investigated a triple cocktail therapy with XAV939, ICAT (a compound targeting the $\beta$-catenin/p300 complex), and niclosamide (targeting the cascade at several levels) in human uterine leiomyoma cells. The combination exhibited antitumor effects in vitro by disrupting proliferation of cancer cells.

E7449, another TNK inhibitor, was shown to be slightly less potent compared with XAV939, but to target other players (such as PARPs) and to work in parallel in conferring antitumor effects (McGonigle et al., 2015). WXL-8, a nitro-substituted derivative of XAV939, has been shown to possess improved antiproliferative activity in hepatic cancer cells, both in vivo and in vitro, whereas it led to stabilization of both axin isoforms and a complete removal of $\beta$-catenin from within the nucleus (Ma et al., 2015). Similar results have been demonstrated with other novel TNK inhibitors, such as JW67, JW74 (Waaler et al., 2011), and JW55 (Waaler et al., 2012). Nathubhai et al. (2017) showed that when compounds 13 and 14 are compared with XAV939, they exhibit 1) higher potency (approximately $10 \times$ ) since they can both display strong antiproliferative activity in DLD-1 colon cancer cells at low concentrations (at which XAV939 is inactive) and 2) isoform selectivity that could imply fewer off-target effects. The highly potent WNT inhibitor kinase inhibitor 4 (WIKI4), a new tool in the TNK inhibitory arsenal, was recently proposed as another potential target. WIKI4 regulates axin degradation, actually by inhibiting axin's ubiquitination, in a variety of cell lines and it is positioned as a potential WNT inhibitory candidate for further investigation (James et al., 2012). G007-LK was shown to inhibit WNT signaling and plays a particularly important role in pathologies where the decisive factor is an APC mutation (e.g., in many human cancers). The compound exhibits a $50 \%$ attenuation of APC mutation-related WNT signaling (Lau et al., 2013); however, gastrointestinal tract toxicity might be a restricting factor in G007-LK's wider use.

AZ1366 is another TNK inhibitor that produces antitumor effects, in combination with an EGFR inhibitor, in non-small cell lung cancer cells, mediated via effects on axin. The compound induced the stabilization of axin 1 and thus the upregulation of $\beta$-catenin target genes (c-myc, axin2, survivin), but loss of axin1 rendered AZ1366 ineffective, implying that axin1 is required for the action of the molecule (Scarborough et al., 2017). This finding was challenged by Quackenbush et al. (2016) in a mouse colorectal model. They showed that when the TNK inhibitor was coadministered with irinotecanin, on the one hand axin2 levels were induced and the treatment led to attenuation of tumor growth; however, there was no change in $\beta$-catenin or c-myc levels. This finding makes the association between WNT inhibition and the anti-tumor effects exhibited by AZ1366 improbable and it seems that other nonWNT-related TNK-mediated actions might be involved (Quackenbush et al., 2016).

PORCN IWP inhibitors were extensively discussed in section A of this chapter. Nonetheless, IWRs might also be acting via axin2 stabilization (Chen et al., 2009a). Lastly, NVP-TNKS656 (Shultz et al., 2013) and compounds 9 and 25 (Johannes et al., 2015) were described to have favorable pharmacokinetic properties that could be used in further preclinical investigations (the first two for oral and the latter for intravenous administration).

There is also a number of compounds that target axin without belonging to the TNK inhibitor class. The group of Salerno has repeatedly shown that the inhibitor SEN461 can stabilize axin1, leading to suppressed 
$\beta$-catenin activity. The findings of the authors have been translated to an antitumor effect of SEN461 against glioblastoma (De Robertis et al., 2013) and sarcoma (De Robertis et al., 2014). An induction of levels of axin1 (but not axin2) is correlated with the mode of action of tigecycline, which has been shown to confer beneficial effects in cervical cancer when given in combination with paclitaxel in a mouse xenograft model (Li et al., 2015). Nevertheless, an effect of the tigecycline-mediated antitumor activity on other signaling pathways (such as PI3K/Akt) cannot be excluded (Zhong et al., 2016). Lastly, SKL2001 is a compound selected by screening of approximately 270,000 molecules and was shown to possess agonist properties. These are mediated mainly via a disruption of the axin/ $\beta$-catenin interaction by competing with axin for binding to $\beta$-catenin, leading to activation of the WNT cascade. Interestingly, SKL2001 does not affect the phosphorylation status of GSK3 $\beta$ (Gwak et al., 2012).

3. Interventions Targeting CK1. Pyrvinium is an anthelminthic drug that was shown to allosterically activate CK1 $\alpha$. Thorne et al. (2010) identified CK1 $\alpha$ as the main target of pyrvinium, albeit effects were shown toward all CK1 isoforms and also secondarily on pygopus. Saraswati et al. (2010) confirmed the CK1 $\alpha$ involvement of pyrvinium treatment in vitro and showed that the compound can inhibit WNT signaling and confer beneficial effects following ischemia in a mouse MI model. Nevertheless, a high number (approximately 59\%) of intracardially injected mice demonstrated sudden death without an etiology for the pyrvinium-mediated cardiac toxicity being evident. Venerando et al. (2013) challenged the CK1 $\alpha$ theory and proposed an alternative mechanism of pyrvinium's actions via an effect on PI3K/Akt, whereas Harada et al. (2012) indicated a STAT3-mediated mechanism. Furthermore, it is possible that other CK1 isoforms (other than the $\mathrm{CK} 1 \alpha$ ) could be targeted to attenuate the destruction complex or act at other components upstream in the signaling cascade (Del Valle-Perez et al., 2011). CKI-7 blocked CK (as well as DVL but not $\beta$-catenin) in Xenopus (Peters et al., 1999). Kim et al. (2010b) demonstrated that inhibiting CK1 $\varepsilon$ with the specific inhibitory compound IC261 led to a suppression of $\beta$-catenin transcription. A study with IC261 and selective inhibitors for CK1 $\delta / \varepsilon$ (PF670462) and CK1 $\varepsilon$ (PF4800567) implied that they could all inhibit the signaling pathway; however, IC261 might also possess activity elsewhere, such as inhibition of microtubule polymerization leading to halting of mitosis (Cheong et al., 2011). More recently, Laco et al. (2015) showed that the p38 MAPK inhibitor SB203580 might be inhibiting CK1 $1 \delta / \varepsilon$, which was proposed to play a crucial role in mediating changes in pluripotent stem cells differentiating to cardiomyocytes. SR-3029 (a potent CK $1 \delta / \varepsilon$ inhibitor) robustly inhibited CK1 $\delta$ and was very effective against several forms of human breast cancer (in vivo data) (Rosenberg et al., 2015). CK1 $\gamma$ targeting does not appear to affect the $\beta$-catenin destruction complex. Nevertheless, CK1 $\gamma$ is associated with LRP6, thus its pharmacological targeting could lead to suppression of WNT signaling (Davidson et al., 2005).

4. Interventions Activating GSK3 $\beta$. GSK $3 \beta$ plays a key role in many cellular functions and in the mechanisms controlling cell cycle, gene expression, protein translation, apoptosis, etc. Furthermore, GSK3 $\beta$ plays a prominent role in the regulation of several signaling pathways, such as PI3K/mTOR, WNT, MAPK, etc. (Phukan et al., 2010).

Curcumin is a naturally occurring compound, broadly used in Indian cuisine. It has been associated with antioxidant, anti-inflammatory, as well as antitumor activities. Its anticancer effects have been extensively studied in a wide range of human cancer cell lines and it is noteworthy that at the point of the preparation of this review paper, 583 papers on "curcumin in cancer" were identified in PubMed. This compound affects various transcription factors, receptors, kinases, inflammatory cells, microRNAs, and different signaling pathways, including the Wnt signaling pathway. It generally exhibits its anti-WNT signaling pathway effects at the level of transcription, by blockade of STAT, NF- $\kappa$ B, and AP-1-related transcription factors (Kasi et al., 2016). However, Choi et al. (2010) showed that GSK3 $\beta$ might also be implicated. The theory proposed by the authors was that curcumin affects Akt expression, which inhibits phosphorylation of GSK3 $\beta$, thereby inducing the destruction complex to reduce $\beta$-catenin's stabilization and translocation into the nucleus. Nevertheless, this mode of action of curcumin is not very well understood, and more studies are required to reveal its actual effects on GSK3 $\beta$ (Choi et al., 2010). Interestingly, although the orchestrated collaboration of GSK $3 \beta$, axin, CK1 $\alpha$, and APC have been shown to be decisive for the formation of the destructive complex, Ohishi et al. (2015) attempted to challenge the theory. They showed that an alkaloid compound called 9-hydroxycanthin-6one attenuated $\mathrm{WNT} / \beta$-catenin signaling by activating GSK $3 \beta$, an effect that is not dependent on CK1 $\alpha$ phosphorylation. All-trans retinoic acid (ATRA) promoted GSK3 $\beta$, leading to a disruption of $\beta$-catenin levels in hepatic cancer stem cells and demonstrates maximum efficacy when coadministered with docetaxel (making tumor cells more sensitive to chemotherapy) (Zhu et al., 2015). Surprisingly, the nonsteroidal antiinflammatory $S$-ibuprofen induces the phosphorylated form of GSK3 $\beta$, which in turn suppresses NF- $\kappa$ B and the $\beta$-catenin target genes in cancer cells (Greenspan et al., 2011).

5. Interventions Inhibiting GSK3ß. It was only approximately 20 years ago that Klein and Melton (1996) reported GSK3 $\beta$ to be a target of $\mathrm{LiCl}$, the wellestablished drug treatment of bipolar disorder. What is more fascinating is that $\mathrm{LiCl}$ does not generally inhibits 
other kinases (e.g., MAPK, ERK, etc.), although crosstalking mechanisms between GSK3, Akt/mTOR, and HIF-1 $\alpha$ do exist (Mottet et al., 2003). It is remarkable that in many WNT-related assays, $\mathrm{LiCl}$ is regarded as a positive control for the activation of $\mathrm{WNT} / \beta$-catenin signaling. The association between $\mathrm{LiCl}$ and the $\beta$-catenin cascade is further strengthened by the findings of Wexler et al. (2008). They showed that LiCl can stimulate the WNT cascade and induce proliferation in adult hippocampal progenitor cells, but inhibition of $\beta$-catenin by RNAi counteracts the effects of $\mathrm{LiCl}$. Valproic acid, another agent routinely used in modern psychopharmacology, has also been shown to target GSK3 $\beta$ (Chen et al., 1999). Human neuroblastoma SH-SY5Y cells were treated with valproic acid, which led to a robust inhibition of GSK3 $\beta$ and an increase in levels of $\beta$-catenin both in cytosol and in the nucleus. A report by Hall et al. (2002) suggested that valproate inhibits GSK3 $\beta$ indirectly via an unknown mechanism in developing neurons, in contrast to LiCl, which has been shown to act directly in developing neurons. Nevertheless, Ryves and Harwood (2001) implied that the ability of valproate (and $\mathrm{LiCl}$ ) to exhibit inhibitory effects on GSK3 $\beta$ depends largely on the cell type or particular areas of the brain, a finding showing that our understanding of the effects of mood stabilizers on the GSK $3 \beta$ of the mammalian brain are far from complete.

6-Bromoindirubin-3'-oxime (BIO) is a specific and competitive inhibitor of GSK3 $\beta$ that has been shown to exert important effects on the differentiation and viability of cells, thus having an important role to play in regenerative medicine strategies. BIO was shown to induce neovascularization both in vitro and in vivo (Kohler et al., 2014). BIO activates the WNT cascade and, when combined with other factors, it improves survival and maintains the undifferentiated status of porcine embryonic germ cells (Wen et al., 2010), as well as of embryonic stem cells (Sato et al., 2004). In addition, the boosting effect it exerts on $\beta$-catenin activity led to a robust amplification of the proliferation of both adult and neonatal cardiomyocytes (Tseng et al., 2006), a very interesting finding in the pursuit for novel treatments of heart disease (e.g., myocardial infarction). In contrast, BIO is found to exert rather noxious effects on human mesenchymal stem cells, because it was shown to inhibit the cell cycle and thus reduce viability of human mesenchymal stem cells preparations (both in vitro and in a mouse model) (Krause et al., 2010). A novel bifunctional compound IBU-PO (taking its name from the diptych ibuprofen and octyl-pyridostigmine of which it consists) is also another compound targeting GSK3 $\beta$. It is a combination of a nonsteroidal antiinflammatory drug and a cholinesterase inhibitor and has been used in Alzheimer's amyloid-B-peptide $(\mathrm{A} \beta)$ in neuronal in vitro cultures as models of Alzheimer's disease. IBU-PO was shown to inhibit GSK3 $\beta$ by inducing its phosphorylation at $\mathrm{Ser}^{9}$ and to stabilize $\beta$-catenin; the consequent activation of the WNT signaling leads to a restoration of $A \beta$-mediated neurotoxicity (Farias et al., 2005). Curcumin appears to be an all-around player, acting on several levels of the WNT cascade, and it was described to inhibit GSK3 $\beta$ and axin in 3T3-L1 cells, leading to an inactivation of the destruction complex, translocation of $\beta$-catenin to the nucleus and activation of the WNT cascade (Ahn et al., 2010), suppressing eventually adipogenesis. SB-216763 and SB-415286 are two molecules that have been described by Coghlan et al. (2000) to possess antiGSK3 properties (inhibiting GSK $3 \alpha$ and $-3 \beta$ with comparable potency). In addition, the authors showed that the selectivity of the two compounds is quite remarkable, because they show minimal activity toward a long list of kinases (including AMPK, CKII, JNK, MAPK, PKA, PKC, etc.). Kenpaullone and SB-216763 were demonstrated to possess the strongest inhibitory effect on GSK3 $\beta$ in human neural progenitors, in contrast with $\mathrm{LiCl}$, valproic acid, and indirubin-3monoxime (Lange et al., 2011).

CHIR 99021 is a small aminopyrimidine derivative that inhibits both GSK3 isoforms; however, it appears to be quite selective for GSK3 without affecting other kinase types. Inhibition of GSK3 $\beta$ leads to the observed induction of $\beta$-catenin stabilization in 3T3-L1 preadipocytes. The activation of WNT signaling is the responsible factor for an inhibition of adipogenesis (Bennett et al., 2002). Similarly, the stabilization of $\beta$-catenin (and especially the induction of c-myc expression) following CHIR 99021 treatment, plays a major role in the maintenance of self-renewal of embryonic stem cells (Ye et al., 2012) and the reprogramming of pluripotent stem cells in combination with other factors (Hou et al., 2013). It should be noted that a comparative in vitro study employing mouse embryonic stem cells, showed that CHIR 99021 demonstrated the highest potency and the lowest toxicity in various concentrations tested (Naujok et al., 2014). The novel GSK3 inhibitor CG0009 phosphorylates GSK3 $\beta$ at Ser ${ }^{9}$ and dephosphorylates it at $\mathrm{Tyr}^{216}$ (both actions leading to inhibition of GSK3 $\beta$ ). When several breast cancer cell lines are treated with CG0009, WNT target genes (e.g., c-myc) are upregulated but cyclin D1 levels are paradoxically suppressed, probably due to cancer cell death (mediated via CG0009), and breast cancer cell growth is robustly attenuated (Kim et al., 2013). Lastly, a peptide called L803-mts was suggested as a potent GSK3 $\beta$ inhibitor. In vitro data showed that it is rather specific for GSK3 (Plotkin et al., 2003), whereas in vivo investigation from the same group revealed an interesting GSK3 $\beta$-mediated antidepressive-like action, which warrants further investigation (Kaidanovich-Beilin et al., 2004).

\section{D. $\beta$-Catenin and Gene Transcription}

Once $\beta$-catenin translocates into the nucleus, it forms complexes with several TCF/LEF transcription factors, 
as well as coactivators (namely p300, CBP, BCL9, Pygo, and others) (Cadigan and Waterman, 2012). Following this crucial step, it drives the transcription of a long list of $\beta$-catenin target genes, including $A X I N 2, C D 44$, $C C N D 1$ (cyclin-D1), $M M P 2 / 9, M Y C, V E G F$, and others (Herbst et al., 2014). The fact that the $\beta$-catenin transcriptional complex plays a key role in a wide range of pathologies (especially in cancer), explains the strong pursuit of new compound intervening with its action.

1. Targeting of the T-cell Factor/Lymphoid Enhancer Factor Transcription Factors. The $\beta$-catenin responsive transcription (CRT) is the critical step prior to the activation of the target genes and is very often the culprit for an abnormally functioning WNT signaling. Thus, inhibitors of CRT (iCRT) might pose as interesting tools in the hunt for strategies to inhibit WNT signaling. Following screening of approximately 15,000 compounds, Gonsalves et al. (2011) identified iCRT3, iCRT5, and iCRT14, which interfere with the $\beta$-catenin/ TCF interaction, inhibit the transcription of WNT target genes, and eradicate cancer cells. Two remarkable findings of the authors were that these three iCRTs: 1 ) were specifically deleterious to human colon tumor biopsy cultures and colorectal cancer cell lines, both demonstrating an abnormally activated WNT signaling pathway and 2) did not appear to have $\beta$-cateninindependent WNT signaling pathway effects. One group has confirmed the WNT-mediated antitumor effect of iCRT3 in acute myeloid leukemia (Griffiths et al., 2015), while others have proposed iCRT5 as a stimulator of T-cell proliferation mediating a beneficial response in dendritic cells without off-target effects (Kafer et al., 2016). In addition, Narayanan et al. (2012) have managed to show as well that (at least some) iCRTs, such as thiazole and oxazole, mediate their effects via $\beta$-catenin/TCF4 interaction and not due to effects on DKK. Quite recently, the diterpenoids NC043 (Wang et al., 2011b), henryin (Li et al., 2013c), and EPLE (Ye et al., 2015) were shown to impair the $\beta$-catenin/TCF association and consequently possess antitumorigenic properties against colon cancer cells (both in vitro and in vivo studies). LF3 is a robust inhibitor of the aforementioned interaction and it led to strong antitumor effects (Fang et al., 2016). Lastly, the compounds code-named BC-21 (Tian et al., 2012) and BC-23 (Zhang et al., 2016a) were proposed as novel inhibitors of the $\beta$-catenin/TCF4 interaction, as shown by studies where they were jointly used with radiation therapy to lung cancer (BC-23) or given as monotherapy to HCT116 colon cancer cells (BC-21). PNU-74654 is another compound that has been shown to interact with the $\beta$-catenin and TCF/LEF complex formation. In vitro experiments demonstrate that PNU-74654 suppresses adrenal steroid synthesis and induces tumor cell apoptosis by attenuating $\beta$-catenin synthesis and accumulation in the nucleus, as well as reducing gene transcription (Leal et al., 2015). Furthermore, PNU-
74654 exhibited cell type selectivity and it was effective against human adrenocortical cell lines but not against human nonadrenal cell lines or mouse adrenal cell lines. The potent compounds PKF115-584 and CGP049090 have been proposed to possess robust WNT inhibitory effects with minimal toxicity. Lepourcelet et al. (2004) showed that these molecules can block the $\beta$-catenin/ TCF transcriptional complex and antagonize exogenous $\beta$-catenin when the latter is injected in embryos of Xenopus, as well as attenuating expression of cyclin D1 and c-myc to cause a halt in the growing of colon cancer cells. As these authors demonstrated, PKF115-584, PKF222-815, and CGP049090 (all of fungal origin) completely inhibited the $\beta$-catenin/TCF4 interaction (Lepourcelet et al., 2004), whereas the first two were also found to prevent the binding of TCF proteins to DNA. The selectivity of these compounds is a matter of further investigation, since PKF115-584 and CGP049090 were also found to inhibit the $\beta$-catenin/ APC complex formation. In addition, PKF115-584 and CGP049090 appeared to be associated with effects on inflammatory components such as tumor necrosis factor $\alpha(\mathrm{TNF}-\alpha)$ and interleukin $1 \beta$ (IL-1 $\beta$ ), also key players in cancer as well as other pathologic conditions (Landman et al., 2013). In addition, the PKF118-310 compound elicited deleterious effects on breast tumor initiating cells and halted their growth in vivo (Hallett et al., 2012). On the other hand, Huang and Shih (2009) reported the compound Daxx to amplify the $\beta$-catenin/ TCF interaction, thus producing inducing effects on WNT signaling pathway.

Several naturally occurring compounds have been associated with the regulation of $\beta$-catenin/TCF interaction. The polyphenol flavonoid quercetin and its derivative, isoquercitrin, have received ample interest. There is evidence for quercetin-mediated antitumor activity from in vitro studies (Matsukawa et al., 1997), as well as studies involving cancer patients not responding to standard therapies (Ferry et al., 1996). Park et al. (2005) used immunoprecipitation studies and demonstrated that quercetin can interfere with the $\beta$-catenin/ TCF4 complex formation in the nucleus. This led to blockade of the WNT signaling pathway in SW480 colon cancer cells, as demonstrated by a suppression of c-myc expression. Isoquercitrin was also found to inhibit the signaling at the level of TCF and to possess antitumor activity against three different colon cancer cell lines, with the additional benefit of lower noncancerous cell toxicity in comparison with the parent molecule quercetin (Amado et al., 2014). Aesculetin, which is a derivative of the anticoagulant coumarin, could also disrupt the $\beta$-catenin/TCF transcriptional complex formation by binding directly to specific residues of $\beta$-catenin $\left(\mathrm{Asn}^{387}, \mathrm{Gly}^{307}, \mathrm{Lys}^{312}\right.$, and $\left.\mathrm{Lys}^{345}\right)$. Thus, aesculetin robustly suppresses the expression of c-myc and cyclin D1, switching the signaling off and conferring antiproliferative and antitumor effects on three 
different cell types of colon cancer (HCT116, HCT15, and DLD1) (Lee et al., 2013). Curcumin is a naturally occurring compound, already mentioned previously (section XI.C.4) The main mode by which curcumin confers its inhibitory effect on WNT signaling pathway is by affecting the $\beta$-catenin/TCF 4 complex formation, and this has been shown in a wide range of cancer cell lines (Jaiswal et al., 2002). Interestingly, apart from TCF4, curcumin was also shown to act at two other levels of the CRT, namely at CBP and p300, leading to suppression of WNT target genes transcription (Teiten et al., 2011) and an inhibition of androgen-dependent prostate cancer cells. What makes curcumin more intriguing as a compound is that it exhibits its effects in part via affecting the WNT signaling/androgen crosstalking. Curcumin inhibits the expression of the androgen receptor (AR) and contributes to the apoptosis of human endometrial carcinoma cells, a phenomenon that appears to be WNT-mediated (since WNT3A can reverse the effect) (Feng et al., 2014). Furthermore, another fascinating point is that according to Teiten et al. (2011), curcumin only has an antiproliferative and antitumor effect against AR-dependent prostate cancers lines, whereas AR-independent ones do not respond to curcumin. In contrast, curcumin is shown to activate the $\mathrm{WNT} / \beta$-catenin signaling pathway and stimulate neurogenesis in neural stem cells (Tiwari et al., 2016) or reverse the effects of dexamethasone on osteoporosis in a rat model (Chen et al., 2016), which increases the notion for a compound that behaves differently according to the cell type or the environment established around or by the cell types themselves.

Lastly, nonsteroidal anti-inflammatory drugs have been suggested as potentially attractive agents interfering with the transcriptional activity of $\beta$-catenin. Aspirin and indomethacin suppress gene transcription of $\beta$-catenin/TCF 4 , without altering the expression of TCF4 itself. The most probable explanation for the observed effects is that aspirin and indomethacin affect the phosphorylation status of $\beta$-catenin and TCF4, leading to inactivation of the complex and subsequently to a robust inhibition of target gene transcription (Dihlmann et al., 2001). Celecoxib and its analog 2,5dimethyl-celecoxib (the latter lacking anti-COX-2 activity) were shown to inhibit TCF7L2 (TCF4) expression in the myocardium in a cardiomyopathy mouse model (Fan et al., 2011) and in human colon cancer (Egashira et al., 2017). Both studies demonstrated that the WNT inhibitory activity of these molecules is not dependent on COX-2 inhibition; however, further investigation is warranted to reveal the precise mode of action in preventing WNT target gene transcription. Lastly, sulindac (a COX-1/2 inhibitor), was also shown to possess antitumor activity by suppressing TCF transcriptional activity (Tinsley et al., 2010; Li et al., $2013 \mathrm{~b}$ ) as well as $\beta$-catenin synthesis (Li et al., 2013b), although other investigators have provided data that sulindac might also be mediating its effects via DVL (Lee et al., 2009).

2. Targeting of CREB Binding Protein. ICG-001 (also known as PRI-724), is a small compound that has been extensively used in basic research and has already been tested in Ib and IIa clinical trials. It is a potent specific inhibitor of the $\beta$-catenin/CBP interaction, acting by binding to the $\mathrm{N}$ terminus of $\mathrm{CBP}$ and leading to suppression of growth-related gene expression. Interestingly, ICG-001 kills mouse xenograft models of colon cancer and colon tumor cells but leaves healthy colon cells unaffected (Emami et al., 2004). ICG-001 acts as a WNT pathway antagonist and robustly prevents fibrosis or reverses its development, while improving animal survival in various pathologies (possibly via effects on [myo]fibroblasts) such as idiopathic pulmonary fibrosis (Henderson et al., 2010). Similar beneficial effects of the ICG-001 were demonstrated in drugresistant acute lymphoblastic leukemia (Gang et al., 2014), urological fibrosis (Zhang et al., 2015a), pancreatic adenocarcinoma (Arensman et al., 2014), multiple myeloma (Grigson et al., 2015), hepatic fibrosis (Osawa et al., 2015), ECM-related lung remodeling (Koopmans et al., 2016), skin fibrosis (Beyer et al., 2013), and quite recently following ischemia in an MI rat model (Sasaki et al., 2013); please refer to section VIII.A for details. In addition, reports from a Phase Ia clinical trial are showing that ICG-001 is, toxicologically, safe in an acceptable level in patients with advanced solid tumors (El-Khoueiry et al., 2013). It should be noted here that combinational therapy of ICG-001 with other compounds (e.g., PKF118 discussed above) is feasible and can produce beneficial effects. Nevertheless, Ayadi et al. (2015), showed that it is possible for these compounds (ICG-001 and PKF118) to possess off-target side effects that might not be directly associated with WNT signaling, and thus making the deeper investigation of the field more urgent than ever. Lastly, the team of Delgado et al. (2014) performed a meticulous search for compounds possessing a structural similarity to ICG-001, examining a database of almost 3.5 million compounds. Following filtering, they eventually narrowed their search to one compound, PMED-1. This compound indeed targets the $\beta$-catenin/CBP complex (just like ICG-001) and was successful in limiting hepatocellular carcinoma but was not deleterious to healthy human hepatocytes. Furthermore, the shorter half-life and fewer off-target effects compared with the parent molecule can be regarded as major advantages but its hydrophobicity is an important disadvantage for its further studying in vivo (Delgado et al., 2014).

3. Targeting of p300. A peptide compound called ICAT was first shown to associate with $\beta$-catenin in both cytoplasm and nucleus and to disrupt the formation of $\beta$-catenin/TCF4 complex in vitro and in vivo (Tago et al., 2000). Daniels and Weis (2002) took it one step further, showing that the full-length peptide 
(81 amino acids long) inhibited the $\beta$-catenin/LEF1/ p300 interaction, whereas its helical domain (ICAT-61) can itself disrupt the binding with p300 but not interfere with the binding of TCF/LEF, proving that ICAT actually possesses two regions leading to antagonism of the pathway. ICAT has been tested as a WNT signaling negative regulator in various studies, encompassing glioma tumors (Zhang et al., 2015b), osteogenesis by stem cells (Kim et al., 2008), and the development of T-cells (Pongracz et al., 2006). Quite recently, a molecule wittily named windorphen (WD) was described (Hao et al., 2013). Studies using zebrafish embryos showed that WD acts downstream of the destruction complex and the level of $\beta$-catenin translocation into the nucleus, leading to profound structural effects on the zebrafish embryo. Furthermore, it appears that WD is selective in targeting the interaction of $\beta$-catenin with p300, although it has no effect whatsoever in the $\beta$-catenin/CBP association (Hao et al., 2013), exactly the opposite effect demonstrated by ICG-001 (Emami et al., 2004). These interesting WNT inhibitory results were extrapolated to cancer. The authors demonstrated with in vitro studies (using cancer cells) that WD's inhibitory effects on WNT signaling lead to the dramatic apoptosis of human colon adenocarcinoma SW480 and RKO cells, as well as of prostate cancer DU145/PC3 cells (which all show an exaggerated WNT signaling activation), but not of human lung cancer H460 cells (which are not associated with WNT signaling activation abnormalities) (Hao et al., 2013). IQ1 is a molecule that is suggested to mediate the $\beta$-catenin/p300 coactivator interaction, during lung morphogenesis in mice. Interestingly, Sasaki and Kahn (2014) demonstrated that pharmacological inhibition of the $\beta$-catenin/p300 interaction by IQ1 actually amplifies the $\beta$-catenin/TCF interaction, thus inducing the signaling pathway in the lungs of mouse embryos, leading to proximalization of the lung epithelium. It is interesting to add here that although curcumin is regarded chiefly to act at the $\beta$-catenin/TCF level (see above), certain of its derivatives, namely bisdemethoxycurcumin and demethoxycurcumin, have been shown to possess p300-mediating activity instead. Ryu et al. (2008) provided evidence for the involvement of bisdemethoxycurcumin and demethoxycurcumin in the downregulation of p300 without any effect on TCF 4 or in the levels of $\beta$-catenin, demonstrating for the first time that the class of curcuminrelated compounds might also act at this level.

4. Targeting of BCL9. Antagonism of the $\beta$-catenin/ BCL9 protein-protein interaction (PPI) complex formation is a very attractive strategy for inhibiting the WNT signaling pathway, for several reasons: 1) BCL9 mediates $\beta$-catenin gene transcription of several pathologic conditions, 2) the aforementioned complex is robustly expressed in tumors but not in healthy cells, and 3) eliminating BCL9/ $\beta$-catenin interaction does not appear to have off-target effects (Takada et al., 2012). de la
Roche et al. (2012) showed that the $\beta$-catenin molecule possesses a weak point, which can be exploited by carnosic acid to degrade $\beta$-catenin in an $\alpha$-helix H1dependent fashion. Carnosic acid was selected after screening a library of a total of more than 45,000 compounds and was found to disrupt binding of $\beta$-catenin to BCL9 and lead to degradation of free (unphosphorylated) $\beta$-catenin, thus blocking the WNT signaling pathway. Mammalian LATS large tumor suppressor (LATS) 2, is a kinase that plays major roles in the Hippo/ YAP signaling pathway (Hoa et al., 2016), mediating especially functions relevant to cell proliferation and oncogenesis. LATS2 is downregulated in various colorectal cancers but studies with immunoprecipitation showed that it directly binds to $\beta$-catenin and disrupts the complexing of the latter with BCL9. Thus, compounds that could target LATS2 could pose as attractive tools for the blockade of WNT signaling and the halting of tumors such as colorectal cancer (Li et al., 2013a). Wisniewski et al. (2016) proposed several small compound inhibitors, attached to piperazine scaffolds (which can form salt bridge interactions with $\beta$-catenin) that show $\beta$-catenin/BCL9 disrupting capabilities. The most promising compound was found to be compound 11 , as it exhibits a surprising selectivity and is also more hydrophilic than the other compounds, while being very effective in halting growth of colorectal cell lines (such as SW480 and HCT116). In the same context, Hoggard et al. (2015) designed several selective compounds attached to a scaffold mimicking the binding of the side chains of an $\alpha$-helix. The orchestrated action of the inhibitors along with the scaffold lead to selective and robust $\beta$-catenin/BCL9 inhibition, as demonstrated by studies using HEK293, SW480 (colorectal cancer), and MDA-MB-231 (breast cancer) cell lines. Lastly, Takada et al. (2012) designed a stabilized $\alpha$-helix of BCL9 called SAH-BCL9, which is shown to selectively disrupt the $\beta$-catenin/BCL9 interaction, inhibit WNT target gene transcription, and possess antitumor activities, as well as effects on angiogenesis and metastasis, without toxic off-target effects, as observed from in vitro and in vivo mouse studies.

\section{E. Conclusions and Future Directions}

In the last decade, there has been a remarkable growth in the number of compounds targeting the Wnt signaling pathway. Based on the mutations identified in different components of the WNT/ $\beta$-catenin pathway in multiple cancers, the majority of these compounds is designed to aim at this branch of the WNT signaling cascade. In cardiovascular conditions, however, alterations in the regulation of the pathway rather than mutations in its components are likely to contribute to the disease process. Therefore, for cardiovascular conditions the preferential sites of intervention in the pathway would be more upstream, at the level of WNT proteins or their interaction with the receptor complex. 
Fortunately, the number of compounds allowing upstream intervention is increasing and several of them have been tested, particularly in models of MI. As discussed in detail in section VIII, interventions in PORCN (WNT-974 and GNF-6231) or at the level of the receptor complex (UM206) showed beneficial effects on infarct healing. Moreover, also interventions aiming at the $\beta$-catenin signaling branch (BIO, ICG-001, and XAV939) improved the wound healing following MI, suggesting an involvement of $\beta$-catenin-mediated signaling; however, this does not exclude the involvement of non- $\beta$-catenin-mediated signaling in infarct healing. Unfortunately, pharmacological interventions have been only sparsely tested in other cardiovascular conditions, so it is recommended to perform drug interventions at different levels of the pathway in these models in the near future, preferably in a single experiment.

A major concern for the global inhibition of WNT signaling is the occurrence of side effects. Several rapidly dividing cells, e.g., in intestinal epithelium and skin, rely on WNT signaling, and an intervention therefore could disturb their normal proliferation, causing, e.g., diarrhea. Surprisingly, such side effects have rarely been observed where these drugs were administered. A potential explanation for this could be that the amount of WNT signaling needed by these epithelial cells is limited, allowing a partial blockade to be without harmful side effects on these cells. Obviously, further research will be needed on this topic before these compounds can be successfully applied in patients. Moreover, these studies should go hand in hand with the development and validation of biomarkers (such as circulating DKK) for WNT signaling activity, to obtain a quick surrogate end point for the effectiveness of the therapy in individual patients.

Recently, several registered drugs and food supplements have been shown to act as inhibitors of the WNT pathway. An obvious advantage of these compounds is that they can be readily applied in man, allowing a rapid translation of preclinical findings to patients. However, a downside is that the dose needed to block WNT signaling usually is quite high, which may give rise to side effects. This problem could be addressed by administering several different drugs at a lower dose. These drugs can be chosen to target the pathway at different levels, which can have an additive (or even synergistic) effect and result in sufficient inhibition of WNT signaling, without the occurrence of severe side effects. On the other hand, the newly developed drugs that target Wnt signaling in a more selective way should be tested and optimized for selectivity, toxicity, and pharmacokinetic profile to develop them toward use in the clinic.

\section{Future Perspectives}

In the last decade, a large number of studies have been published on the characteristics of WNT signaling in general and its role in cardiovascular diseases in particular. In this review, we focused on studies on the role of WNT signaling in cardiovascular conditions as diverse as atherosclerosis, cardiac hypertrophy, and arrhythmias. However, potential common denominators in the pathologic mechanisms underlying these diseases appear to be processes such as cell proliferation and differentiation, inflammation and fibrosis, and the role for WNT signaling in these processes is well established. This helps to explain the regulatory role that WNT signaling plays in these diverse cardiovascular conditions.

Most of the studies presented in this review describe the effect of an intervention in WNT signaling in a cardiovascular disease model. The results are sometimes contradictory with other studies, making it hard to draw firm conclusions regarding the potential therapeutic benefit of the intervention. However, a couple of remarks have to be made. First, in many studies, genetic interventions and deletions of a specific gene in particular have been used. Since the WNT pathway has many homologs at different levels in the signaling cascade, this may give rise to redundancy and adaptation of the organism to the absence of the specific gene. The large number of regulatory mechanisms that can control the signaling activity can further influence the outcomes. Moreover, many gene deletion experiments with little or no phenotype are likely to never have been published in the first place, giving rise to a biased picture. This calls for interventions that can target the signaling pathway in a more general way. Second, experimental details such as age and genetic background of the animals, sex, and method of disease induction can be confounding the results. Third, the timing of the intervention can be critical for the outcome, as, e.g., described in section IX of this review, where inhibition of WNT signaling needs to be carefully timed to achieve optimal cardiomyocyte differentiation.

These considerations clearly indicate that the field is about to become ready for the next leap, where therapeutic interventions in WNT signaling are tested in clinically relevant models of cardiovascular disease or even in patients suffering from cardiovascular diseases. In the past, the major problem was that the options for pharmacological intervention in the pathway were limited or even absent due to the lack of suitable drugs. As can be deduced from section XI, this situation has changed drastically in the last years. Many experimental drugs are now available, targeting the pathway at multiple levels, and some of them are currently tested in man. Moreover, several registered drugs have been shown to target WNT signaling in diseases such as cancer, allowing the testing of the benefit of intervening in WNT signaling in cardiovascular conditions. It is highly likely that these kinds of experiments will teach us more about the feasibility and the benefit of targeting WNT signaling in cardiovascular diseases. 


\section{Authorship Contributions}

Wrote or contributed to the writing of the manuscript: Foulquier, Daskalopoulos, Lluri, Hermans, Deb, Blankesteijn.

\section{References}

Abbott NJ, Patabendige AA, Dolman DE, Yusof SR, and Begley DJ (2010) Structure and function of the blood-brain barrier. Neurobiol Dis 37:13-25.

Adler PN (2012) The frizzled/stan pathway and planar cell polarity in the Drosophila wing. Curr Top Dev Biol 101:1-31.

Ahmad F, Lal H, Zhou J, Vagnozzi RJ, Yu JE, Shang X, Woodgett JR, Gao E, and Force $\mathrm{T}$ (2014) Cardiomyocyte-specific deletion of Gsk3 $\alpha$ mitigates postmyocardial infarction remodeling, contractile dysfunction, and heart failure. J Am Coll Cardiol 64:696-706.

Ahn J, Lee H, Kim S, and Ha T (2010) Curcumin-induced suppression of adipogenic differentiation is accompanied by activation of Wnt/beta-catenin signaling. Am J Physiol Cell Physiol 298:C1510-C1516.

Ahn VE, Chu ML, Choi HJ, Tran D, Abo A, and Weis WI (2011) Structural basis of Wnt signaling inhibition by Dickkopf binding to LRP5/6. Dev Cell 21:862-873.

Ai Z, Fischer A, Spray DC, Brown AM, and Fishman GI (2000) Wnt-1 regulation of connexin43 in cardiac myocytes. J Clin Invest 105:161-171.

Ain QU, Seemab U, Rashid S, Nawaz MS, and Kamal MA (2013) Prediction of structure of human WNT-CRD (FZD) complex for computational drug repurposing. PLoS One 8:e54630.

Aisagbonhi O, Rai M, Ryzhov S, Atria N, Feoktistov I, and Hatzopoulos AK (2011) Experimental myocardial infarction triggers canonical Wnt signaling and endothelial-to-mesenchymal transition. Dis Model Mech 4:469-483.

Alapati D, Rong M, Chen S, Lin C, Li Y, and Wu S (2013) Inhibition of LRP5/6mediated Wnt/ $\beta$-catenin signaling by Mesd attenuates hyperoxia-induced pulmonary hypertension in neonatal rats. Pediatr Res 73:719-725.

Alfaro MP, Pagni M, Vincent A, Atkinson J, Hill MF, Cates J, Davidson JM, Rottman J, Lee E, and Young PP (2008) The Wnt modulator sFRP2 enhances mesenchymal stem cell engraftment, granulation tissue formation and myocardial repair. Proc Natl Acad Sci USA 105:18366-18371.

Amado NG, Predes D, Fonseca BF, Cerqueira DM, Reis AH, Dudenhoeffer AC, Borges HL, Mendes FA, and Abreu JG (2014) Isoquercitrin suppresses colon cancer cell growth in vitro by targeting the Wnt/ß-catenin signaling pathway. J Biol Chem 289:35456-35467.

Ambrosy AP, Fonarow GC, Butler J, Chioncel O, Greene SJ, Vaduganathan M, Nodari S, Lam CS, Sato N, Shah AN, et al. (2014) The global health and economic burden of hospitalizations for heart failure: lessons learned from hospitalized heart failure registries. $J$ Am Coll Cardiol 63:1123-1133.

An WF, Germain AR, Bishop JA, Nag PP, Metkar S, Ketterman J, Walk M, Weiwer M, Liu X, Patnaik D, et al. (2010). Discovery of potent and highly selective inhibitors of GSK3b probe reports from the NIH molecular libraries program, National Center for Biotechnology Information (US), Bethesda, MD.

Anton R, Chatterjee SS, Simundza J, Cowin P, and Dasgupta R (2011) A systematic screen for micro-RNAs regulating the canonical Wnt pathway. PLoS One 6:e26257.

Anton R, Kestler HA, and Kühl M (2007) Beta-catenin signaling contributes to stemness and regulates early differentiation in murine embryonic stem cells. FEBS Lett 581:5247-5254.

Antos CL, McKinsey TA, Frey N, Kutschke W, McAnally J, Shelton JM, Richardson JA, Hill JA, and Olson EN (2002) Activated glycogen synthase-3 beta suppresses cardiac hypertrophy in vivo. Proc Natl Acad Sci USA 99:907-912.

Arensman MD, Telesca D, Lay AR, Kershaw KM, Wu N, Donahue TR, and Dawson DW (2014) The CREB-binding protein inhibitor ICG-001 suppresses pancreatic cancer growth. Mol Cancer Ther 13:2303-2314.

Artus C, Glacial F, Ganeshamoorthy K, Ziegler N, Godet M, Guilbert T, Liebner S, and Couraud PO (2014) The Wnt/planar cell polarity signaling pathway contributes to the integrity of tight junctions in brain endothelial cells. J Cereb Blood Flow Metab 34:433-440.

Askevold ET, Aukrust P, Nymo SH, Lunde IG, Kaasbøll OJ, Aakhus S, Florholmen G, Ohm IK, Strand ME, Attramadal H, et al.; GISSI-HF Investigators (2014) The cardiokine secreted Frizzled-related protein 3, a modulator of Wnt signalling, in clinical and experimental heart failure. J Intern Med 275:621-630.

Askevold ET, Gullestad L, Nymo S, Kjekshus J, Yndestad A, Latini R, Cleland JG, McMurray JJ, Aukrust P, and Ueland T (2015) Secreted frizzled related protein 3 in chronic heart failure: analysis from the Controlled Rosuvastatin Multinational Trial in Heart Failure (CORONA). PLoS One 10:e133970.

Ayadi M, Bouygues A, Ouaret D, Ferrand N, Chouaib S, Thiery JP, Muchardt C, Sabbah M, and Larsen AK (2015) Chronic chemotherapeutic stress promote evolution of stemness and WNT/beta-catenin signaling in colorectal cancer cells: implications for clinical use of WNT-signaling inhibitors. Oncotarget 6 18518-18533.

Azzolin L, Panciera T, Soligo S, Enzo E, Bicciato S, Dupont S, Bresolin S, Frasson C, Basso G, Guzzardo V, et al. (2014) YAP/TAZ incorporation in the $\beta$-catenin destruction complex orchestrates the Wnt response. Cell 158:157-170.

Bader M and Ganten D (2008) Update on tissue renin-angiotensin systems. $J$ Mol Med (Berl) 86:615-621.

Bao MW, Cai Z, Zhang XJ, Li L, Liu X, Wan N, Hu G, Wan F, Zhang R, Zhu X, et al. (2015) Dickkopf-3 protects against cardiac dysfunction and ventricular remodelling following myocardial infarction. Basic Res Cardiol 110:25.

Barandon L, Casassus F, Leroux L, Moreau C, Allières C, Lamazière JM, Dufourcq P, Couffinhal T, and Duplàa C (2011) Secreted frizzled-related protein-1 improves postinfarction scar formation through a modulation of inflammatory response. Arterioscler Thromb Vasc Biol 31:e80-e87.

Barandon L, Couffinhal T, Ezan J, Dufourcq P, Costet P, Alzieu P, Leroux L, Moreau C, Dare D, and Duplàa C (2003) Reduction of infarct size and prevention of cardiac rupture in transgenic mice overexpressing FrzA. Circulation 108:2282-2289.
Barker N, van Es JH, Kuipers J, Kujala P, van den Born M, Cozijnsen M, Haegebarth A, Korving J, Begthel H, Peters PJ, et al. (2007) Identification of stem cells in small intestine and colon by marker gene Lgr5. Nature 449:1003-1007.

Barrott JJ, Cash GM, Smith AP, Barrow JR, and Murtaugh LC (2011) Deletion of mouse Porcn blocks Wnt ligand secretion and reveals an ectodermal etiology of human focal dermal hypoplasia/Goltz syndrome. Proc Natl Acad Sci USA 108: 12752-12757.

Bartscherer K, Pelte N, Ingelfinger D, and Boutros M (2006) Secretion of Wnt ligands requires Evi, a conserved transmembrane protein. Cell 125:523-533.

Basso C, Bauce B, Corrado D, and Thiene G (2011) Pathophysiology of arrhythmogenic cardiomyopathy. Nat Rev Cardiol 9:223-233.

Bastakoty D, Saraswati S, Joshi P, Atkinson J, Feoktistov I, Liu J, Harris JL, and Young PP (2016) Temporary, systemic inhibition of the WNT/beta-catenin pathway promotes regenerative cardiac repair following myocardial infarct. Cell Stem Cells Regen Med 2 DOI:10.16966/2472-6990.111.

Baurand A, Zelarayan L, Betney R, Gehrke C, Dunger S, Noack C, Busjahn A, Huelsken J, Taketo MM, Birchmeier W, et al. (2007) Beta-catenin downregulation is required for adaptive cardiac remodeling. Circ Res 100:1353-1362.

Beazley KE, Nurminsky D, Lima F, Gandhi C, and Nurminskaya MV (2015) Wnt16 attenuates TGF $\beta$-induced chondrogenic transformation in vascular smooth muscle. Arterioscler Thromb Vasc Biol 35:573-579.

Belema Bedada F, Technau A, Ebelt H, Schulze M, and Braun T (2005) Activation of myogenic differentiation pathways in adult bone marrow-derived stem cells. $\mathrm{Mol}$ Cell Biol 25:9509-9519.

Beljaars L, Daliri S, Dijkhuizen C, Poelstra K, and Gosens R (2017) WNT-5A regulates TGF- $\beta$-related activities in liver fibrosis. Am $J$ Physiol Gastrointest Liver Physiol 312:G219-G227.

Benjamin EJ, Blaha MJ, Chiuve SE, Cushman M, Das SR, Deo R, de Ferranti SD, Floyd J, Fornage M, Gillespie C, et al.; American Heart Association Statistics Committee and Stroke Statistics Subcommittee (2017) Heart disease and stroke statistics-2017 update: a report from the American Heart Association. Circulation 135:e146-e603.

Bennett CN, Ross SE, Longo KA, Bajnok L, Hemati N, Johnson KW, Harrison SD, and MacDougald OA (2002) Regulation of Wnt signaling during adipogenesis. $J$ Biol Chem 277:30998-31004.

Benzler J, Andrews ZB, Pracht C, Stöhr S, Shepherd PR, Grattan DR, and Tups A (2013) Hypothalamic WNT signalling is impaired during obesity and reinstated by leptin treatment in male mice. Endocrinology 154:4737-4745.

Berger W, Meindl A, van de Pol TJ, Cremers FP, Ropers HH, Döerner C, Monaco A, Bergen AA, Lebo R, Warburg M, et al. (1992) Isolation of a candidate gene for Norrie disease by positional cloning. Nat Genet 1:199-203.

Beyer C, Reichert H, Akan H, Mallano T, Schramm A, Dees C, Palumbo-Zerr K, Lin NY, Distler A, Gelse K, et al. (2013) Blockade of canonical Wnt signalling ameliorates experimental dermal fibrosis. Ann Rheum Dis 72:1255-1258.

Bhat RA, Stauffer B, Komm BS, and Bodine PV (2007) Structure-function analysis of secreted frizzled-related protein-1 for its Wnt antagonist function. J Cell Biochem 102:1519-1528.

Bhatt PM and Malgor R (2014) Wnt5a: a player in the pathogenesis of atherosclerosis and other inflammatory disorders. Atherosclerosis 237:155-162.

Blankesteijn WM, Essers-Janssen YP, Ulrich MM, and Smits JF (1996) Increased expression of a homologue of drosophila tissue polarity gene "frizzled" in left ventricular hypertrophy in the rat, as identified by subtractive hybridization. $J$ Mol Cell Cardiol 28:1187-1191.

Blankesteijn WM, Essers-Janssen YP, Verluyten MJ, Daemen MJ, and Smits JF (1997) A homologue of Drosophila tissue polarity gene frizzled is expressed in migrating myofibroblasts in the infarcted rat heart. Nat Med 3:541-544.

Blankesteijn WM, van de Schans VA, ter Horst P, and Smits JF (2008) The Wnt/ frizzled/GSK-3 beta pathway: a novel therapeutic target for cardiac hypertrophy. Trends Pharmacol Sci 29:175-180.

Blyszczuk P, Müller-Edenborn B, Valenta T, Osto E, Stellato M, Behnke S, Glatz K, Basler K, Lüscher TF, Distler O, et al. (2017) Transforming growth factor- $\beta$-dependent Wnt secretion controls myofibroblast formation and myocardial fibrosis progression in experimental autoimmune myocarditis. Eur Heart $J$ 38:1413-1425.

Bodine PV, Stauffer B, Ponce-de-Leon H, Bhat RA, Mangine A, Seestaller-Wehr LM Moran RA, Billiard J, Fukayama S, Komm BS, et al. (2009) A small molecule inhibitor of the Wnt antagonist secreted frizzled-related protein-1 stimulates bone formation. Bone 44:1063-1068

Bond J, Sedmera D, Jourdan J, Zhang Y, Eisenberg CA, Eisenberg LM, and Gourdie RG (2003) Wnt11 and Wnt7a are up-regulated in association with differentiation of cardiac conduction cells in vitro and in vivo. Dev Dyn 227:536-543.

Borrell-Pages M, Carolina Romero J, and Badimon L (2015a) LRP5 and plasma cholesterol levels modulate the canonical Wnt pathway in peripheral blood leukocytes. Immunol Cell Biol 93:653-661.

Borrell-Pages M, Romero JC, and Badimon L (2014a) Cholesterol modulates LRP5 expression in the vessel wall. Atherosclerosis 235:363-370.

Borrell-Pagès M, Romero JC, and Badimon L (2014b) LRP5 negatively regulates differentiation of monocytes through abrogation of Wnt signalling. J Cell Mol Med 18:314-325.

Borrell-Pagès M, Romero JC, and Badimon L (2015b) LRP5 deficiency downregulates Wnt signalling and promotes aortic lipid infiltration in hypercholesterolaemic mice. J Cell Mol Med 19:770-777.

Borrell-Pages M, Romero JC, Crespo J, Juan-Babot O, and Badimon L (2016a) LRP5 associates with specific subsets of macrophages: molecular and functional effects. $J$ Mol Cell Cardiol 90:146-156.

Borrell-Pagès M, Romero JC, Juan-Babot O, and Badimon L (2011) Wnt pathway activation, cell migration, and lipid uptake is regulated by low-density lipoprotein receptor-related protein 5 in human macrophages. Eur Heart J 32:2841-2850.

Borrell-Pages M, Vilahur G, Romero JC, Casaní L, Bejar MT, and Badimon L (2016b) LRP5/canonical Wnt signalling and healing of ischemic myocardium. Basic Res Cardiol 111:67. 
Bovolenta P, Esteve P, Ruiz JM, Cisneros E, and Lopez-Rios J (2008) Beyond Wnt inhibition: new functions of secreted Frizzled-related proteins in development and disease. J Cell Sci 121:737-746.

Boyden LM, Mao J, Belsky J, Mitzner L, Farhi A, Mitnick MA, Wu D, Insogna K, and Lifton RP (2002) High bone density due to a mutation in LDL-receptor-related protein 5. N Engl J Med 346:1513-1521.

Brault V, Moore R, Kutsch S, Ishibashi M, Rowitch DH, McMahon AP, Sommer L, Boussadia O, and Kemler R (2001) Inactivation of the beta-catenin gene by Wnt1Cre-mediated deletion results in dramatic brain malformation and failure of craniofacial development. Development 128:1253-1264.

Braz JC, Gill RM, Corbly AK, Jones BD, Jin N, Vlahos CJ, Wu Q, and Shen W (2009) Selective activation of PI3Kalpha/Akt/GSK-3beta signalling and cardiac compensatory hypertrophy during recovery from heart failure. Eur J Heart Fail 11:739-748.

Bretón-Romero R, Feng B, Holbrook M, Farb MG, Fetterman JL, Linder EA, Berk BD, Masaki N, Weisbrod RM, Inagaki E, et al. (2016) Endothelial Dysfunction in Human Diabetes Is Mediated by Wnt5a-JNK Signaling. Arterioscler Thromb Vasc Biol 36:561-569.

Brunt KR, Zhang Y, Mihic A, Li M, Li SH, Xue P, Zhang W, Basmaji S, Tsang K, Weisel RD, et al. (2012) Role of WNT/ $\beta$-catenin signaling in rejuvenating myogenic differentiation of aged mesenchymal stem cells from cardiac patients. Am J Pathol 181:2067-2078.

Buckingham M, Meilhac S, and Zaffran S (2005) Building the mammalian heart from two sources of myocardial cells. Nat Rev Genet 6:826-835.

Buechling T, Bartscherer K, Ohkawara B, Chaudhary V, Spirohn K, Niehrs C, and Boutros M (2010) Wnt/Frizzled signaling requires dPRR, the Drosophila homolog of the prorenin receptor. Curr Biol 20:1263-1268.

Burgos-Morón E, Calderón-Montaño JM, Salvador J, Robles A, and López-Lázaro M (2010) The dark side of curcumin. Int J Cancer 126:1771-1775.

Cadigan KM and Waterman ML (2012) TCF/LEFs and Wnt signaling in the nucleus. Cold Spring Harb Perspect Biol 4:a007906.

Cai T, Sun D, Duan Y, Wen P, Dai C, Yang J, and He W (2016) WNT/ß-catenin signaling promotes VSMCs to osteogenic transdifferentiation and calcification through directly modulating Runx2 gene expression. Exp Cell Res 345:206-217.

Caira FC, Stock SR, Gleason TG, McGee EC, Huang J, Bonow RO, Spelsberg TC, McCarthy PM, Rahimtoola SH, and Rajamannan NM (2006) Human degenerative valve disease is associated with up-regulation of low-density lipoprotein receptorrelated protein 5 receptor-mediated bone formation. J Am Coll Cardiol 47:1707-1712

Camici GG, Schiavoni M, Francia P, Bachschmid M, Martin-Padura I, Hersberger M, Tanner FC, Pelicci P, Volpe M, Anversa P, et al. (2007) Genetic deletion of p66(Shc) adaptor protein prevents hyperglycemia-induced endothelial dysfunction and oxidative stress. Proc Natl Acad Sci USA 104:5217-5222.

Carmon KS and Loose DS (2008) Wnt7a interaction with Fzd5 and detection of signaling activation using a split eGFP. Biochem Biophys Res Commun 368 $285-291$.

Carmon KS and Loose DS (2010) Development of a bioassay for detection of Wntbinding affinities for individual frizzled receptors. Anal Biochem 401:288-294.

Carron C, Pascal A, Djiane A, Boucaut JC, Shi DL, and Umbhauer M (2003) Frizzled receptor dimerization is sufficient to activate the Wnt/beta-catenin pathway. $J$ Cell Sci 116:2541-2550.

Carthy JM, Garmaroudi FS, Luo Z, and McManus BM (2011) Wnt3a induces myofibroblast differentiation by upregulating TGF- $\beta$ signaling through SMAD2 in a $\beta$-catenin-dependent manner. PLoS One 6:e19809.

Castoldi G, Redaelli S, van de Greef WM, di Gioia CR, Busca G, Sperti G, and Stella A (2005) Angiotensin II modulates frizzled-2 receptor expression in rat vascular smooth muscle cells. Clin Sci (Lond) 108:523-530.

Cerutti C, Kurdi M, Bricca G, Hodroj W, Paultre C, Randon J, and Gustin MP (2006) Transcriptional alterations in the left ventricle of three hypertensive rat models. Physiol Genomics 27:295-308.

Chablais F, Veit J, Rainer G, and Jaźwińska A (2011) The zebrafish heart regenerates after cryoinjury-induced myocardial infarction. BMC Dev Biol 11:21.

Chelko SP, Asimaki A, Andersen P, Bedja D, Amat-Alarcon N, DeMazumder D, Jasti R, MacRae CA, Leber R, Kleber AG, et al. (2016) Central role for GSK3 $\beta$ in the pathogenesis of arrhythmogenic cardiomyopathy. JCI Insight 1:e85923.

Chen B, Dodge ME, Tang W, Lu J, Ma Z, Fan CW, Wei S, Hao W, Kilgore J, Williams NS, et al. (2009a) Small molecule-mediated disruption of Wnt-dependent signaling in tissue regeneration and cancer. Nat Chem Biol 5:100-107.

Chen G, Huang LD, Jiang YM, and Manji HK (1999) The mood-stabilizing agent valproate inhibits the activity of glycogen synthase kinase-3. J Neurochem 72 $1327-1330$

Chen L, Wu Q, Guo F, Xia B, and Zuo J (2004) Expression of Dishevelled-1 in wound healing after acute myocardial infarction: possible involvement in myofibroblast proliferation and migration. J Cell Mol Med 8:257-264.

Chen M, Wang J, Lu J, Bond MC, Ren XR, Lyerly HK, Barak LS, and Chen W (2009b) The anti-helminthic niclosamide inhibits Wnt/Frizzled1 signaling. Biochemistry 48:10267-10274.

Chen S, Bubeck D, MacDonald BT, Liang WX, Mao JH, Malinauskas T, Llorca O, Aricescu AR, Siebold C, He X, et al. (2011) Structural and functional studies of LRP6 ectodomain reveal a platform for Wnt signaling. Dev Cell 21:848-861.

Chen VC, Stull R, Joo D, Cheng X, and Keller G (2008) Notch signaling respecifies the hemangioblast to a cardiac fate. Nat Biotechnol 26:1169-1178.

Chen W, ten Berge D, Brown J, Ahn S, Hu LA, Miller WE, Caron MG, Barak LS, Nusse R, and Lefkowitz RJ (2003) Dishevelled 2 recruits beta-arrestin 2 to mediate Wnt5A-stimulated endocytosis of Frizzled 4. Science 301:1391-1394.

Chen X, Shevtsov SP, Hsich E, Cui L, Haq S, Aronovitz M, Kerkelä R, Molkentin JD, Liao R, Salomon RN, et al. (2006) The beta-catenin/T-cell factor/lymphocyte enhancer factor signaling pathway is required for normal and stress-induced cardiac hypertrophy. Mol Cell Biol 26:4462-4473.

Chen Y, Zhang Y, Tang J, Liu F, Hu Q, Luo C, Tang J, Feng H, and Zhang JH (2015) Norrin protected blood-brain barrier via frizzled-4/ $\beta$-catenin pathway after subarachnoid hemorrhage in rats. Stroke 46:529-536.
Chen Z, Xue J, Shen T, Mu S, and Fu Q (2016) Curcumin alleviates glucocorticoidinduced osteoporosis through the regulation of the Wnt signaling pathway. Int $J$ Mol Med 37:329-338.

Cheng CW, Smith SK, and Charnock-Jones DS (2003) Wnt-1 signaling inhibits human umbilical vein endothelial cell proliferation and alters cell morphology. Exp Cell Res 291:415-425.

Cheng CW, Yeh JC, Fan TP, Smith SK, and Charnock-Jones DS (2008a) Wnt5amediated non-canonical Wnt signalling regulates human endothelial cell proliferation and migration. Biochem Biophys Res Commun 365:285-290.

Cheng H, Woodgett J, Maamari M, and Force T (2011a) Targeting GSK-3 family members in the heart: a very sharp double-edged sword. J Mol Cell Cardiol 51 $607-613$

Cheng SL, Ramachandran B, Behrmann A, Shao JS, Mead M, Smith C, Krchma K, Bello Arredondo Y, Kovacs A, Kapoor K, et al. (2015) Vascular smooth muscle LRP6 limits arteriosclerotic calcification in diabetic LDLR-/- mice by restraining noncanonical Wnt signals. Circ Res 117:142-156.

Cheng SL, Shao JS, Cai J, Sierra OL, and Towler DA (2008b) Msx2 exerts bone anabolism via canonical Wnt signaling. J Biol Chem 283:20505-20522.

Cheng Z, Biechele T, Wei Z, Morrone S, Moon RT, Wang L, and Xu W (2011b) Crystal structures of the extracellular domain of LRP6 and its complex with DKK1. Nat Struct Mol Biol 18:1204-1210.

Cheong JK, Nguyen TH, Wang H, Tan P, Voorhoeve PM, Lee SH, and Virshup DM (2011) IC261 induces cell cycle arrest and apoptosis of human cancer cells via $\mathrm{CK} 1 \delta / \varepsilon$ and $\mathrm{Wnt} / \beta$-catenin independent inhibition of mitotic spindle formation. Oncogene 30:2558-2569.

Choi HY, Lim JE, and Hong JH (2010) Curcumin interrupts the interaction between the androgen receptor and Wnt/ $\beta$-catenin signaling pathway in LNCaP prostate cancer cells. Prostate Cancer Prostatic Dis 13:343-349.

Choi S, Gustafson-Wagner EA, Wang Q, Harlan SM, Sinn HW, Lin JL, and Lin JJ (2007) The intercalated disk protein, mXinalpha, is capable of interacting with beta-catenin and bundling actin filaments [corrected]. J Biol Chem 282: 36024-36036.

Chong JM, Uren A, Rubin JS, and Speicher DW (2002) Disulfide bond assignments of secreted Frizzled-related protein-1 provide insights about Frizzled homology and netrin modules. J Biol Chem 277:5134-5144.

Christman MA II, Goetz DJ, Dickerson E, McCall KD, Lewis CJ, Benencia F, Silver MJ, Kohn LD, and Malgor R (2008) Wnt5a is expressed in murine and human atherosclerotic lesions. Am J Physiol Heart Circ Physiol 294:H2864-H2870.

Clerk A, Cullingford TE, Fuller SJ, Giraldo A, Markou T, Pikkarainen S, and Sugden PH (2007) Signaling pathways mediating cardiac myocyte gene expression in physiological and stress responses. J Cell Physiol 212:311-322.

Clevers H and Nusse R (2012) Wnt/ß-catenin signaling and disease. Cell 149 1192-1205.

Cochain C and Zernecke A (2017) Macrophages in vascular inflammation and atherosclerosis. Pflugers Arch 469:485-499.

Coghlan MP, Culbert AA, Cross DA, Corcoran SL, Yates JW, Pearce NJ, Rausch OL, Murphy GJ, Carter PS, Roxbee Cox L, et al. (2000) Selective small molecule inhibitors of glycogen synthase kinase-3 modulate glycogen metabolism and gene transcription. Chem Biol 7:793-803.

Cohen ED, Miller MF, Wang Z, Moon RT, and Morrisey EE (2012) Wnt5a and Wnt11 are essential for second heart field progenitor development. Development 139 1931-1940.

Cohen P and Goedert M (2004) GSK3 inhibitors: development and therapeutic potential. Nat Rev Drug Discov 3:479-487.

Colvin KL and Yeager ME (2014) Animal models of pulmonary hypertension matching disease mechanisms to etiology of the human disease. $J$ Pulm Respir Med 4:198.

Cook D, Fry MJ, Hughes K, Sumathipala R, Woodgett JR, and Dale TC (1996) Wingless inactivates glycogen synthase kinase-3 via an intracellular signalling pathway which involves a protein kinase C. EMBO $J$ 15:4526-4536.

Corada M, Morini MF, and Dejana E (2014) Signaling pathways in the specification of arteries and veins. Arterioscler Thromb Vasc Biol 34:2372-2377.

Courboulin A, Paulin R, Giguère NJ, Saksouk N, Perreault T, Meloche J, Paquet ER, Biardel S, Provencher S, Côté J, et al. (2011) Role for miR-204 in human pulmonary arterial hypertension. J Exp Med 208:535-548.

Courtwright A, Siamakpour-Reihani S, Arbiser JL, Banet N, Hilliard E, Fried L, Livasy C, Ketelsen D, Nepal DB, Perou CM, et al. (2009) Secreted frizzle-related protein 2 stimulates angiogenesis via a calcineurin/NFAT signaling pathway. Cancer Res 69:4621-4628.

Croce JC and McClay DR (2008) Evolution of the Wnt pathways. Methods Mol Biol 469:3-18.

Cruciat CM and Niehrs C (2013) Secreted and transmembrane wnt inhibitors and activators. Cold Spring Harb Perspect Biol 5:a015081.

Cruciat CM, Ohkawara B, Acebron SP, Karaulanov E, Reinhard C, Ingelfinger D, Boutros M, and Niehrs C (2010) Requirement of prorenin receptor and vacuolar $H$ +-ATPase-mediated acidification for Wnt signaling. Science 327:459-463.

Daneman R, Agalliu D, Zhou L, Kuhnert F, Kuo CJ, and Barres BA (2009) Wnt/betacatenin signaling is required for CNS, but not non-CNS, angiogenesis. Proc Nat Acad Sci USA 106:641-646.

Daniels DL and Weis WI (2002) ICAT inhibits beta-catenin binding to Tcf/Lef-family transcription factors and the general coactivator p300 using independent structural modules. Mol Cell 10:573-584.

Dann CE, Hsieh JC, Rattner A, Sharma D, Nathans J, and Leahy DJ (2001) Insights into Wnt binding and signalling from the structures of two Frizzled cysteine-rich domains. Nature 412:86-90.

Daskalopoulos EP, Janssen BJ, and Blankesteijn WM (2012) Myofibroblasts in the infarct area: concepts and challenges. Microsc Microanal 18:35-49.

Davidson G, Wu W, Shen J, Bilic J, Fenger U, Stannek P, Glinka A, and Niehrs C (2005) Casein kinase 1 gamma couples Wnt receptor activation to cytoplasmic signal transduction. Nature 438:867-872. 
Dawson K, Aflaki M, and Nattel S (2013) Role of the Wnt-Frizzled system in cardiac pathophysiology: a rapidly developing, poorly understood area with enormous potential. J Physiol 591:1409-1432.

de Gasparo M, Catt KJ, Inagami T, Wright JW, and Unger T (2000) International union of pharmacology. XXIII. The angiotensin II receptors. Pharmacol Rev 52: $415-472$

de Jesus Perez V, Yuan K, Alastalo TP, Spiekerkoetter E, and Rabinovitch M (2014) Targeting the Wnt signaling pathways in pulmonary arterial hypertension. Drug Discov Today 19:1270-1276.

de Jesus Perez VA, Alastalo TP, Wu JC, Axelrod JD, Cooke JP, Amieva M, and Rabinovitch M (2009) Bone morphogenetic protein 2 induces pulmonary angiogenesis via Wnt-beta-catenin and Wnt-RhoA-Rac1 pathways. J Cell Biol 184: $83-99$

de la Roche M, Rutherford TJ, Gupta D, Veprintsev DB, Saxty B, Freund SM, and Bienz M (2012) An intrinsically labile $\alpha$-helix abutting the BCL9-binding site of $\beta$-catenin is required for its inhibition by carnosic acid. Nat Commun 3:680.

de Lau W, Barker N, Low TY, Koo BK, Li VS, Teunissen H, Kujala P, Haegebarth A Peters PJ, van de Wetering M, et al. (2011) Lgr5 homologues associate with Wnt receptors and mediate R-spondin signalling. Nature 476:293-297.

de Lau W, Peng WC, Gros P, and Clevers H (2014) The R-spondin/Lgr5/Rnf43 module: regulator of Wnt signal strength. Genes Dev 28:305-316.

De Robertis A, Mennillo F, Rossi M, Valensin S, Tunici P, Mori E, Caradonna N, Varrone M, and Salerno M (2014) Human sarcoma growth is sensitive to smallmolecule mediated AXIN stabilization. PLoS One 9:e97847.

De Robertis A, Valensin S, Rossi M, Tunici P, Verani M, De Rosa A, Giordano C, Varrone M, Nencini A, Pratelli C, et al. (2013) Identification and characterization of a small-molecule inhibitor of Wnt signaling in glioblastoma cells. Mol Cancer Ther 12:1180-1189.

Deb A (2014) Cell-cell interaction in the heart via Wnt/ $\beta$-catenin pathway after cardiac injury. Cardiovasc Res 102:214-223.

Deb A, Davis BH, Guo J, Ni A, Huang J, Zhang Z, Mu H, and Dzau VJ (2008) SFRP2 regulates cardiomyogenic differentiation by inhibiting a positive transcriptional autofeedback loop of Wnt3a. Stem Cells 26:35-44.

Dejana E (2010) The role of wnt signaling in physiological and pathological angiogenesis. Circ Res 107:943-952.

Del Valle-Pérez B, Arqués O, Vinyoles M, de Herreros AG, and Duñach M (2011) Coordinated action of CK1 isoforms in canonical Wnt signaling. Mol Cell Biol 31: $2877-2888$

Delgado ER, Yang J, So J, Leimgruber S, Kahn M, Ishitani T, Shin D, Mustata Wilson G, and Monga SP (2014) Identification and characterization of a novel small-molecule inhibitor of $\beta$-catenin signaling. Am J Pathol 184:2111-2122.

Demer LL and Tintut Y (2008) Vascular calcification: pathobiology of a multifaceted disease. Circulation 117:2938-2948.

Descamps B, Sewduth R, Ferreira Tojais N, Jaspard B, Reynaud A, Sohet F, Lacolley $\mathrm{P}$, Allières $\mathrm{C}$, Lamazière $\mathrm{JM}$, Moreau $\mathrm{C}$, et al. (2012) Frizzled 4 regulates arterial network organization through noncanonical Wnt/planar cell polarity signaling. Circ Res 110:47-58.

Dhore CR, Cleutjens JP, Lutgens E, Cleutjens KB, Geusens PP, Kitslaar PJ, Tordoir JH, Spronk HM, Vermeer C, and Daemen MJ (2001) Differential expression of bone matrix regulatory proteins in human atherosclerotic plaques. Arterioscler Thromb Vasc Biol 21:1998-2003.

Dihlmann S, Siermann A, and von Knebel Doeberitz M (2001) The nonsteroidal antiinflammatory drugs aspirin and indomethacin attenuate beta-catenin/TCF-4 signaling. Oncogene 20:645-653.

Dijksterhuis JP, Petersen J, and Schulte G (2014) WNT/Frizzled signalling: receptorligand selectivity with focus on FZD-G protein signalling and its physiological relevance: IUPHAR Review 3. Br J Pharmacol 171:1195-1209.

DiRenzo DM, Chaudhary MA, Shi X, Franco SR, Zent J, Wang K, Guo LW, and Kent KC (2016) A crosstalk between TGF- $\beta /$ Smad3 and Wnt/ $\beta$-catenin pathways promotes vascular smooth muscle cell proliferation. Cell Signal 28:498-505.

Dravid G, Ye Z, Hammond H, Chen G, Pyle A, Donovan P, Yu X, and Cheng L (2005) Defining the role of Wnt/beta-catenin signaling in the survival, proliferation, and self-renewal of human embryonic stem cells. Stem Cells 23:1489-1501.

Duan J, Gherghe C, Liu D, Hamlett E, Srikantha L, Rodgers L, Regan JN, Rojas M, Willis M, Leask A, et al. (2012) Wnt1/Bcatenin injury response activates the epicardium and cardiac fibroblasts to promote cardiac repair. $E M B O J \mathbf{3 1}$ : $429-442$

Egashira I, Takahashi-Yanaga F, Nishida R, Arioka M, Igawa K, Tomooka K Nakatsu Y, Tsuzuki T, Nakabeppu Y, Kitazono T, et al. (2017) Celecoxib and 2,5-dimethylcelecoxib inhibit intestinal cancer growth by suppressing the Wnt/ $\beta$-catenin signaling pathway. Cancer Sci 108:108-115.

Egger-Adam D and Katanaev VL (2010) The trimeric G protein Go inflicts a double impact on axin in the Wnt/frizzled signaling pathway. Dev Dyn 239:168-183.

Eken SM, Jin H, Chernogubova E, Li Y, Simon N, Sun C, Korzunowicz G, Busch A Bäcklund A, Österholm C, et al. (2017) MicroRNA-210 enhances fibrous cap stability in advanced atherosclerotic lesions. Circ Res 120:633-644.

El-Khoueiry AB, Ning Y, Yang D, Cole S, Kahn M, Zoghbi M, Berg J, Fujimori M Inada T, Kouji H, et al. (2013) A phase I first-in-human study of PRI-724 in patients (pts) with advanced solid tumors. J Clin Oncol 31 (Suppl):2501.

El Jamali A, Freund C, Rechner C, Scheidereit C, Dietz R, and Bergmann MW (2004) Reoxygenation after severe hypoxia induces cardiomyocyte hypertrophy in vitro: activation of CREB downstream of GSK3beta. FASEB $J$ 18:1096-1098

Emami KH, Nguyen C, Ma H, Kim DH, Jeong KW, Eguchi M, Moon RT, Teo JL, Kim HY, Moon SH, et al. (2004) A small molecule inhibitor of beta-catenin/CREB binding protein transcription [corrected]. Proc Natl Acad Sci USA 101: 12682-12687.

Etheridge SL, Ray S, Li S, Hamblet NS, Lijam N, Tsang M, Greer J, Kardos N, Wang J, Sussman DJ, et al. (2008) Murine dishevelled 3 functions in redundant pathways with dishevelled 1 and 2 in normal cardiac outflow tract, cochlea, and neural tube development. PLoS Genet 4:e1000259.
Ettenberg SA, Charlat O, Daley MP, Liu S, Vincent KJ, Stuart DD, Schuller AG, Yuan J, Ospina B, Green J, et al (2010) Inhibition of tumorigenesis driven by different Wnt proteins requires blockade of distinct ligand-binding regions by LRP6 antibodies. Proc Natl Acad Sci USA 107:15473-15478.

Ezan J, Leroux L, Barandon L, Dufourcq P, Jaspard B, Moreau C, Allières C, Daret D, Couffinhal T, and Duplàa C (2004) FrzA/sFRP-1, a secreted antagonist of the Wnt-Frizzled pathway, controls vascular cell proliferation in vitro and in vivo. Cardiovasc Res 63:731-738.

Fagiani E and Christofori G (2013) Angiopoietins in angiogenesis. Cancer Lett 328: $18-26$

Fan X, Takahashi-Yanaga F, Morimoto S, Zhan DY, Igawa K, Tomooka K, and Sasaguri T (2011) Celecoxib and 2,5-dimethyl-celecoxib prevent cardiac remodeling inhibiting Akt-mediated signal transduction in an inherited dilated cardiomyopathy mouse model. J Pharmacol Exp Ther 338:2-11.

Fang L, Zhu Q, Neuenschwander M, Specker E, Wulf-Goldenberg A, Weis WI, von Kries JP, and Birchmeier W (2016) A small-molecule antagonist of the $\beta$-catenin TCF4 interaction blocks the self-renewal of cancer stem cells and suppresses tumorigenesis. Cancer Res 76:891-901.

Fang M, Alfieri CM, Hulin A, Conway SJ, and Yutzey KE (2014) Loss of $\beta$-catenin promotes chondrogenic differentiation of aortic valve interstitial cells. Arterioscler Thromb Vasc Biol 34:2601-2608.

Farías GG, Godoy JA, Vázquez MC, Adani R, Meshulam H, Avila J, Amitai G, and Inestrosa NC (2005) The anti-inflammatory and cholinesterase inhibitor bifunctional compound IBU-PO protects from beta-amyloid neurotoxicity by acting on Wnt signaling components. Neurobiol Dis 18:176-183.

Felkin LE, Lara-Pezzi EA, Hall JL, Birks EJ, and Barton PJ (2011) Reverse remodelling and recovery from heart failure are associated with complex patterns of gene expression. J Cardiovasc Transl Res 4:321-331.

Feng W, Yang CX, Zhang L, Fang Y, and Yan M (2014) Curcumin promotes the apoptosis of human endometrial carcinoma cells by downregulating the expression of androgen receptor through Wnt signal pathway. Eur J Gynaecol Oncol 35: $718-723$

Ferry DR, Smith A, Malkhandi J, Fyfe DW, deTakats PG, Anderson D, Baker J, and Kerr DJ (1996) Phase I clinical trial of the flavonoid quercetin: pharmacokinetics and evidence for in vivo tyrosine kinase inhibition. Clin Cancer Res 2 659-668.

Fischer MM, Yen W-C, Zheng C, Henner R, Cattaruzza F, Tang T, Yeung P, Biswas T, Lewicki J, Gurney A, et al. (2015) Wnt pathway antagonist ipafricept (FZD8-Fc, OMP-54F28) inhibits tumor growth and reduces tumor-initiating cell frequency in ovarian patient-derived xenograft models, in Proceedings of the 106th Annual Meeting of the American Association for Cancer Research; 2015 April 18-22; Philadelphia, PA.

Foord SM, Bonner TI, Neubig RR, Rosser EM, Pin JP, Davenport AP, Spedding M, and Harmar AJ (2005) International Union of Pharmacology. XLVI. G proteincoupled receptor list. Pharmacol Rev 57:279-288.

Franco CA, Liebner S, and Gerhardt H (2009) Vascular morphogenesis: a Wnt for every vessel? Curr Opin Genet Dev 19:476-483.

Franzeck FC, Hof D, Spescha RD, Hasun M, Akhmedov A, Steffel J, Shi Y, Cosentino F, Tanner FC, von Eckardstein A, et al. (2012) Expression of the aging gene p66Shc is increased in peripheral blood monocytes of patients with acute coronary syndrome but not with stable coronary artery disease. Atherosclerosis 220:282-286.

Freise C, Kretzschmar N, and Querfeld U (2016) Wnt signaling contributes to vascular calcification by induction of matrix metalloproteinases. BMC Cardiovasc Disord 16:185

Fujii N, You L, Xu Z, Uematsu K, Shan J, He B, Mikami I, Edmondson LR, Neale G, Zheng J, et al. (2007) An antagonist of dishevelled protein-protein interaction suppresses beta-catenin-dependent tumor cell growth. Cancer Res 67:573-579.

Fukukawa C, Hanaoka H, Nagayama S, Tsunoda T, Toguchida J, Endo K, Nakamura $\mathrm{Y}$, and Katagiri T (2008) Radioimmunotherapy of human synovial sarcoma using a monoclonal antibody against FZD10. Cancer Sci 99:432-440.

Fulciniti M, Tassone P, Hideshima T, Vallet S, Nanjappa P, Ettenberg SA, Shen Z Patel N, Tai YT, Chauhan D, et al. (2009) Anti-DKK1 mAb (BHQ880) as a potential therapeutic agent for multiple myeloma. Blood 114:371-379.

Galli LM, Barnes T, Cheng T, Acosta L, Anglade A, Willert K, Nusse R, and Burrus LW (2006) Differential inhibition of Wnt-3a by Sfrp-1, Sfrp-2, and Sfrp-3. Dev Dyn 235:681-690.

Gammons MV, Renko M, Johnson CM, Rutherford TJ, and Bienz M (2016) Wnt signalosome assembly by DEP domain swapping of dishevelled. Mol Cell 64 92-104.

Gang EJ, Hsieh YT, Pham J, Zhao Y, Nguyen C, Huantes S, Park E, Naing K, Klemm L, Swaminathan S, et al. (2014) Small-molecule inhibition of CBP/catenin interactions eliminates drug-resistant clones in acute lymphoblastic leukemia. Oncogene 33:2169-2178.

Garcia-Gras E, Lombardi R, Giocondo MJ, Willerson JT, Schneider MD, Khoury DS, and Marian AJ (2006) Suppression of canonical Wnt/beta-catenin signaling by nuclear plakoglobin recapitulates phenotype of arrhythmogenic right ventricular cardiomyopathy. J Clin Invest 116:2012-2021.

Garriock RJ, D'Agostino SL, Pilcher KC, and Krieg PA (2005) Wnt11-R, a protein closely related to mammalian Wnt11, is required for heart morphogenesis in Xenopus. Dev Biol 279:179-192.

Generoso SF, Giustiniano M, La Regina G, Bottone S, Passacantilli S, Di Maro S, Cassese H, Bruno A, Mallardo M, Dentice M, et al. (2015) Pharmacological folding chaperones act as allosteric ligands of Frizzled4. Nat Chem Biol 11:280-286.

Gessert S and Kühl M (2010) The multiple phases and faces of wnt signaling during cardiac differentiation and development. Circ Res 107:186-199.

Gessert S, Maurus D, Brade T, Walther P, Pandur P, and Kühl M (2008) DM-GRASP/ ALCAM/CD166 is required for cardiac morphogenesis and maintenance of cardiac identity in first heart field derived cells. Dev Biol 321:150-161.

Gillers BS, Chiplunkar A, Aly H, Valenta T, Basler K, Christoffels VM, Efimov IR, Boukens BJ, and Rentschler S (2015) Canonical wnt signaling regulates 
atrioventricular junction programming and electrophysiological properties. Circ Res 116:398-406.

Gilmour DF (2015) Familial exudative vitreoretinopathy and related retinopathies. Eye (Lond) 29:1-14.

Gitler AD, Lu MM, Jiang YQ, Epstein JA, and Gruber PJ (2003) Molecular markers of cardiac endocardial cushion development. Dev Dyn 228:643-650.

Glinka A, Wu W, Delius H, Monaghan AP, Blumenstock C, and Niehrs C (1998) Dickkopf- 1 is a member of a new family of secreted proteins and functions in head induction. Nature 391:357-362.

Gong Y, Bourhis E, Chiu C, Stawicki S, DeAlmeida VI, Liu BY, Phamluong K, Cao TC, Carano RA, Ernst JA, et al. (2010) Wnt isoform-specific interactions with coreceptor specify inhibition or potentiation of signaling by LRP6 antibodies. PLoS One 5:e12682.

Gong Y, Slee RB, Fukai N, Rawadi G, Roman-Roman S, Reginato AM, Wang H, Cundy T, Glorieux FH, Lev D, et al.; Osteoporosis-Pseudoglioma Syndrome Collaborative Group (2001) LDL receptor-related protein 5 (LRP5) affects bone accrual and eye development. Cell 107:513-523.

Gonsalves FC, Klein K, Carson BB, Katz S, Ekas LA, Evans S, Nagourney R, Cardozo T, Brown AM, and DasGupta R (2011) An RNAi-based chemical genetic screen identifies three small-molecule inhibitors of the Wnt/wingless signaling pathway. Proc Natl Acad Sci USA 108:5954-5963.

Goodwin AM, Kitajewski J, and D'Amore PA (2007) Wnt1 and Wnt5a affect endothelial proliferation and capillary length; Wnt2 does not. Growth Factors 25:25-32

Gore AV, Swift MR, Cha YR, Lo B, McKinney MC, Li W, Castranova D, Davis A, Mukouyama YS, and Weinstein BM (2011) Rspo1/Wnt signaling promotes angiogenesis via Vegfc/Vegfr3. Development 138:4875-4886.

Grandy D, Shan J, Zhang X, Rao S, Akunuru S, Li H, Zhang Y, Alpatov I, Zhang XA, Lang RA, et al. (2009) Discovery and characterization of a small molecule inhibitor of the PDZ domain of dishevelled. J Biol Chem 284:16256-16263.

Green J, Nusse R, and van Amerongen R (2014) The role of Ryk and Ror receptor tyrosine kinases in Wnt signal transduction. Cold Spring Harb Perspect Biol 6: a009175

Greenspan EJ, Madigan JP, Boardman LA, and Rosenberg DW (2011) Ibuprofen inhibits activation of nuclear beta-catenin in human colon adenomas and induces the phosphorylation of GSK-3beta. Cancer Prev Res (Phila) 4:161-171.

Griffiths EA, Golding MC, Srivastava P, Povinelli BJ, James SR, Ford LA, Wetzler M, Wang ES, and Nemeth MJ (2015) Pharmacological targeting of $\beta$-catenin in normal karyotype acute myeloid leukemia blasts. Haematologica 100:e49-e52.

Grigoryan T, Wend P, Klaus A, and Birchmeier W (2008) Deciphering the function of canonical Wnt signals in development and disease: conditional loss- and gain-offunction mutations of beta-catenin in mice. Genes Dev 22:2308-2341.

Grigson ER, Ozerova M, Pisklakova A, Liu H, Sullivan DM, and Nefedova Y (2015) Canonical Wnt pathway inhibitor ICG-001 induces cytotoxicity of multiple myeloma cells in Wnt-independent manner. PLoS One 10:e117693.

Gross JC, Chaudhary V, Bartscherer K, and Boutros M (2012) Active Wnt proteins are secreted on exosomes. Nat Cell Biol 14:1036-1045.

Guan S, Wang Z, Xin F, and Xin H (2014) Wnt5a is associated with the differentiation of bone marrow mesenchymal stem cells in vascular calcification by connecting with different receptors. Mol Med Rep 10:1985-1991.

Gubb D and García-Bellido A (1982) A genetic analysis of the determination of cuticular polarity during development in Drosophila melanogaster. J Embryol Exp Morphol 68:37-57.

Guettler S (2016) AXIN shapes tankyrase architecture. Structure 24:1625-1627.

Guo X, Ramirez A, Waddell DS, Li Z, Liu X, and Wang XF (2008) Axin and GSK3control Smad3 protein stability and modulate TGF- signaling. Genes Dev 22 $106-120$

Guo X and Wang XF (2009) Signaling cross-talk between TGF-beta/BMP and other pathways. Cell Res 19:71-88.

Gurney A, Axelrod F, Bond CJ, Cain J, Chartier C, Donigan L, Fischer M, Chaudhari A, Ji M, Kapoun AM, et al. (2012) Wnt pathway inhibition via the targeting of Frizzled receptors results in decreased growth and tumorigenicity of human tumors. Proc Natl Acad Sci USA 109:11717-11722.

Gwak J, Hwang SG, Park HS, Choi SR, Park SH, Kim H, Ha NC, Bae SJ, Han JK, Kim DE, et al. (2012) Small molecule-based disruption of the Axin/ $\beta$-catenin protein complex regulates mesenchymal stem cell differentiation. Cell Res 22 237-247.

Haegel H, Larue L, Ohsugi M, Fedorov L, Herrenknecht K, and Kemler R (1995) Lack of beta-catenin affects mouse development at gastrulation. Development 121 3529-3537.

Hagenmueller M, Riffel JH, Bernhold E, Fan J, Katus HA, and Hardt SE (2014) Dapper-1 is essential for Wnt5a induced cardiomyocyte hypertrophy by regulating the Wnt/PCP pathway. FEBS Lett 588:2230-2237.

Hagenmueller M, Riffel JH, Bernhold E, Fan J, Zhang M, Ochs M, Steinbeisser H, Katus HA, and Hardt SE (2013) Dapper-1 induces myocardial remodeling through activation of canonical Wnt signaling in cardiomyocytes. Hypertension 61: 1177-1183.

Hahn JY, Cho HJ, Bae JW, Yuk HS, Kim KI, Park KW, Koo BK, Chae IH, Shin CS, $\mathrm{Oh} \mathrm{BH}$, et al. (2006) Beta-catenin overexpression reduces myocardial infarct size through differential effects on cardiomyocytes and cardiac fibroblasts. J Biol Chem 281:30979-30989.

Hall AC, Brennan A, Goold RG, Cleverley K, Lucas FR, Gordon-Weeks PR, and Salinas PC (2002) Valproate regulates GSK-3-mediated axonal remodeling and synapsin I clustering in developing neurons. Mol Cell Neurosci 20:257-270.

Halleskog C and Schulte G (2013) Pertussis toxin-sensitive heterotrimeric G( $\alpha \mathrm{i} / \mathrm{o})$ proteins mediate WNT/ $\beta$-catenin and WNT/ERK1/2 signaling in mouse primary microglia stimulated with purified WNT-3A. Cell Signal 25:822-828.

Hallett RM, Kondratyev MK, Giacomelli AO, Nixon AM, Girgis-Gabardo A, Ilieva D, and Hassell JA (2012) Small molecule antagonists of the Wnt/ $\beta$-catenin signaling pathway target breast tumor-initiating cells in a Her2/Neu mouse model of breast cancer. PLoS One 7:e33976.
Hamblet NS, Lijam N, Ruiz-Lozano P, Wang J, Yang Y, Luo Z, Mei L, Chien KR, Sussman DJ, and Wynshaw-Boris A (2002) Dishevelled 2 is essential for cardiac outflow tract development, somite segmentation and neural tube closure. Development 129:5827-5838.

Hao HX, Xie Y, Zhang Y, Charlat O, Oster E, Avello M, Lei H, Mickanin C, Liu D, Ruffner H, et al. (2012) ZNRF3 promotes Wnt receptor turnover in an R-spondinsensitive manner. Nature 485:195-200.

Hao J, Ao A, Zhou L, Murphy CK, Frist AY, Keel JJ, Thorne CA, Kim K, Lee E, and Hong CC (2013) Selective small molecule targeting $\beta$-catenin function discovered by in vivo chemical genetic screen. Cell Reports 4:898-904.

Haq S, Choukroun G, Lim H, Tymitz KM, del Monte F, Gwathmey J, Grazette L, Michael A, Hajjar R, Force T, et al. (2001) Differential activation of signal transduction pathways in human hearts with hypertrophy versus advanced heart failure. Circulation 103:670-677.

Haq S, Michael A, Andreucci M, Bhattacharya K, Dotto P, Walters B, Woodgett J, Kilter H, and Force T (2003) Stabilization of beta-catenin by a Wnt-independent mechanism regulates cardiomyocyte growth. Proc Natl Acad Sci USA 100 $4610-4615$

Harada Y, Ishii I, Hatake K, and Kasahara T (2012) Pyrvinium pamoate inhibits proliferation of myeloma/erythroleukemia cells by suppressing mitochondrial respiratory complex I and STAT3. Cancer Lett 319:83-88.

Hardt SE and Sadoshima J (2002) Glycogen synthase kinase-3beta: a novel regulator of cardiac hypertrophy and development. Circ Res 90:1055-1063.

Harris ES and Nelson WJ (2010) Adenomatous polyposis coli regulates endothelial cell migration independent of roles in beta-catenin signaling and cell-cell adhesion. Mol Biol Cell 21:2611-2623.

Harris TJ and Peifer M (2005) Decisions, decisions: beta-catenin chooses between adhesion and transcription. Trends Cell Biol 15:234-237.

Harterink M and Korswagen HC (2012) Dissecting the Wnt secretion pathway: key questions on the modification and intracellular trafficking of Wnt proteins. Acta Physiol (Oxf) 204:8-16.

Hartmann C (2006) A Wnt canon orchestrating osteoblastogenesis. Trends Cell Biol 16:151-158.

Haÿ E, Buczkowski T, Marty C, Da Nascimento S, Sonnet P, and Marie PJ (2012) Peptide-based mediated disruption of $\mathrm{N}$-cadherin-LRP5/6 interaction promotes Wnt signaling and bone formation. J Bone Miner Res 27:1852-1863.

He J, Cai Y, Luo LM, and Wang R (2015) Expression of Wnt and NCX1 and its correlation with cardiomyocyte apoptosis in mouse with myocardial hypertrophy. Asian Pac J Trop Med 8:930-936.

He W, Zhang L, Ni A, Zhang Z, Mirotsou M, Mao L, Pratt RE, and Dzau VJ (2010) Exogenously administered secreted frizzled related protein 2 (Sfrp2) reduces fibrosis and improves cardiac function in a rat model of myocardial infarction. Proc Natl Acad Sci USA 107:21110-21115.

He Z, Li H, Zuo S, Pasha Z, Wang Y, Yang Y, Jiang W, Ashraf M, and Xu M (2011) Transduction of Wnt11 promotes mesenchymal stem cell transdifferentiation into cardiac phenotypes. Stem Cells Dev 20:1771-1778.

Hecht E, Freise C, Websky KV, Nasser H, Kretzschmar N, Stawowy P, Hocher B, and Querfeld U (2016) The matrix metalloproteinases 2 and 9 initiate uraemic vascular calcifications. Nephrol Dial Transplant 31:789-797.

Henderson WR Jr, Chi EY, Ye X, Nguyen C, Tien YT, Zhou B, Borok Z, Knight DA and Kahn M (2010) Inhibition of Wnt/beta-catenin/CREB binding protein (CBP) signaling reverses pulmonary fibrosis. Proc Natl Acad Sci USA 107:14309-14314.

Heo J, Ahn EK, Jeong HG, Kim YH, Leem SH, Lee SJ, Park EK, and Yang M (2013) Transcriptional characterization of Wnt pathway during sequential hepatic differentiation of human embryonic stem cells and adipose tissue-derived stem cells. Biochem Biophys Res Commun 434:235-240.

Herbst A, Jurinovic V, Krebs S, Thieme SE, Blum H, Göke B, and Kolligs FT (2014) Comprehensive analysis of $\beta$-catenin target genes in colorectal carcinoma cell lines with deregulated Wnt/ $\beta$-catenin signaling. BMC Genomics 15:74.

Hermans KC and Blankesteijn WM (2015) Wnt signaling in cardiac disease. Compr Physiol 5:1183-1209.

Hermans KC, Daskalopoulos EP, and Blankesteijn WM (2016) The Janus face of myofibroblasts in the remodeling heart. J Mol Cell Cardiol 91:35-41.

Hermle T, Saltukoglu D, Grünewald J, Walz G, and Simons M (2010) Regulation of Frizzled-dependent planar polarity signaling by a V-ATPase subunit. Curr Biol 20: $1269-1276$

Herrington W, Lacey B, Sherliker P, Armitage J, and Lewington S (2016) Epidemiology of atherosclerosis and the potential to reduce the global burden of atherothrombotic disease. Circ Res 118:535-546.

Hirabayashi Y, Itoh Y, Tabata H, Nakajima K, Akiyama T, Masuyama N, and Gotoh $\mathrm{Y}$ (2004) The Wnt/beta-catenin pathway directs neuronal differentiation of cortical neural precursor cells. Development 131:2791-2801.

Hirotani S, Zhai P, Tomita H, Galeotti J, Marquez JP, Gao S, Hong C, Yatani A, Avila $J$, and Sadoshima $J$ (2007) Inhibition of glycogen synthase kinase 3beta during heart failure is protective. Circ Res 101:1164-1174.

Hoa L, Kulaberoglu Y, Gundogdu R, Cook D, Mavis M, Gomez M, Gomez V, and Hergovich A (2016) The characterisation of LATS2 kinase regulation in HippoYAP signalling. Cell Signal 28:488-497.

Hoang B, Moos M Jr, Vukicevic S, and Luyten FP (1996) Primary structure and tissue distribution of FRZB, a novel protein related to Drosophila frizzled, suggest a role in skeletal morphogenesis. J Biol Chem 271:26131-26137.

Hoggard LR, Zhang Y, Zhang M, Panic V, Wisniewski JA, and Ji H (2015) Rational design of selective small-molecule inhibitors for $\beta$-catenin/B-cell lymphoma 9 protein-protein interactions. J Am Chem Soc 137:12249-12260.

Holderfield MT and Hughes CC (2008) Crosstalk between vascular endothelial growth factor, notch, and transforming growth factor-beta in vascular morphogenesis. Circ Res 102:637-652.

Hou N, Ye B, Li X, Margulies KB, Xu H, Wang X, and Li F (2016) Transcription factor 7-like 2 mediates canonical Wnt/ $\beta$-catenin signaling and c-myc upregulation in heart failure. Circ Heart Fail 9:e003010. 
Hou P, Li Y, Zhang X, Liu C, Guan J, Li H, Zhao T, Ye J, Yang W, Liu K, et al. (2013) Pluripotent stem cells induced from mouse somatic cells by small-molecule compounds. Science 341:651-654

Hsieh JC, Kodjabachian L, Rebbert ML, Rattner A, Smallwood PM, Samos CH, Nusse R, Dawid IB, and Nathans J (1999) A new secreted protein that binds to Wnt proteins and inhibits their activities. Nature 398:431-436.

Hsieh M, Boerboom D, Shimada M, Lo Y, Parlow AF, Luhmann UF, Berger W, and Richards JS (2005) Mice null for Frizzled4 (Fzd4-/-) are infertile and exhibit impaired corpora lutea formation and function. Biol Reprod 73:1135-1146.

Huang SM, Mishina YM, Liu S, Cheung A, Stegmeier F, Michaud GA, Charlat O, Wiellette E, Zhang Y, Wiessner S, et al. (2009) Tankyrase inhibition stabilizes axin and antagonizes Wnt signalling. Nature 461:614-620.

Huang YS and Shih HM (2009) Daxx positively modulates beta-catenin/TCF4mediated transcriptional potential. Biochem Biophys Res Commun 386:762-768.

Huelsken J, Vogel R, Brinkmann V, Erdmann B, Birchmeier C, and Birchmeier W (2000) Requirement for beta-catenin in anterior-posterior axis formation in mice. $J$ Cell Biol 148:567-578

Hulin A, Moore V, James JM, and Yutzey KE (2017) Loss of Axin2 results in impaired heart valve maturation and subsequent myxomatous valve disease. Cardiovasc Res 113:40-51

Hunter JJ and Chien KR (1999) Signaling pathways for cardiac hypertrophy and failure. $N$ Engl J Med 341:1276-1283.

Imajo M, Miyatake K, Iimura A, Miyamoto A, and Nishida E (2012) A molecular mechanism that links Hippo signalling to the inhibition of Wnt/ $\beta$-catenin signalling. EMBO J 31:1109-1122.

Iyer SP, Beck JT, Stewart AK, Shah J, Kelly KR, Isaacs R, Bilic S, Sen S, and Munshi NC (2014) A Phase IB multicentre dose-determination study of BHQ880 in combination with anti-myeloma therapy and zoledronic acid in patients with relapsed or refractory multiple myeloma and prior skeletal-related events. $\mathrm{Br}$ J Haematol 167:366-375.

Jaiswal AS, Marlow BP, Gupta N, and Narayan S (2002) Beta-catenin-mediated transactivation and cell-cell adhesion pathways are important in curcumin (diferuylmethane)-induced growth arrest and apoptosis in colon cancer cells. Oncogene 21:8414-8427.

James RG, Davidson KC, Bosch KA, Biechele TL, Robin NC, Taylor RJ, Major MB, Camp ND, Fowler K, Martins TJ, et al. (2012) WIKI4, a novel inhibitor of tankyrase and Wnt/ß-catenin signaling. PLoS One 7:e50457.

Jamieson C, Sharma M, and Henderson BR (2014) Targeting the $\beta$-catenin nuclear transport pathway in cancer. Semin Cancer Biol 27:20-29.

Janda CY, Waghray D, Levin AM, Thomas C, and Garcia KC (2012) Structural basis of Wnt recognition by Frizzled. Science 337:59-64.

Jang S, Park JS, and Jeong HS (2015) Neural differentiation of human adipose tissue-derived stem cells involves activation of the Wnt5a/JNK signalling. Stem Cells Int 2015:178618.

Jenei V, Sherwood V, Howlin J, Linnskog R, Säfholm A, Axelsson L, and Andersson T (2009) A t-butyloxycarbonyl-modified Wnt5a-derived hexapeptide functions as a potent antagonist of Wnt5a-dependent melanoma cell invasion. Proc Natl Acad Sci USA 106:19473-19478.

Jian H, Shen X, Liu I, Semenov M, He X, and Wang XF (2006) Smad3-dependent nuclear translocation of beta-catenin is required for TGF-beta1-induced proliferation of bone marrow-derived adult human mesenchymal stem cells. Genes Dev 20:666-674.

Jimeno A, Gordon M, Messersmith WA, Chugh R, Mendelson D, Dupont J, Stagg R, Kapoun AM, Xu L, Brachmann RK, et al. (2014). A first-in-human Phase 1 study of anti-cancer stem cell (CSC) agent OMP-54F28 (FZD8-Fc) targeting the WNT pathway in patients with advanced solid tumors, in Proceedings of American Society of Clinical Oncology (ASCO) Annual Meeting 2014; 2014 May 30-June 3; Chicago, IL. American Society of Clinical Oncology.

Jin X, Guo B, Yan J, Yang R, Chang L, Wang Y, Miao C, Liu S, Zhang H, and Li Y (2015a) Angiotensin II increases secreted frizzled-related protein 5 (sFRP5) expression through AT1 receptor/Rho/ROCK1/JNK signaling in cardiomyocytes. $\mathrm{Mol}$ Cell Biochem 408:215-222.

Jin Y, Wang W, Chai S, Liu J, Yang T, and Wang J (2015b) Wnt5a attenuates hypoxia-induced pulmonary arteriolar remodeling and right ventricular hypertrophy in mice. Exp Biol Med (Maywood) 240:1742-1751.

Johannes JW, Almeida L, Barlaam B, Boriack-Sjodin PA, Casella R, Croft RA, Dishington AP, Gingipalli L, Gu C, Hawkins JL, et al. (2015) Pyrimidinone nicotinamide mimetics as selective tankyrase and wnt pathway inhibitors suitable for in vivo pharmacology. ACS Med Chem Lett 6:254-259.

Joiner DM, Ke J, Zhong Z, Xu HE, and Williams BO (2013) LRP5 and LRP6 in development and disease. Trends Endocrinol Metab 24:31-39.

Jones SE and Jomary C (2002) Secreted frizzled-related proteins: searching for relationships and patterns. BioEssays 24:811-820.

Kadari A, Mekala S, Wagner N, Malan D, Köth J, Doll K, Stappert L, Eckert D, Peitz M, Matthes J, et al. (2015) Robust generation of cardiomyocytes from human iPS cells requires precise modulation of BMP and WNT signaling. Stem Cell Rev 11: $560-569$.

Kaemmerer E and Gassler N (2016) Wnt lipidation and modifiers in intestinal carcinogenesis and cancer. Cancers (Basel) 8:69

Käfer R, Usanova S, Montermann E, Loquai C, Reske-Kunz AB, and Bros M (2016) Inhibitors of $\beta$-catenin affect the immuno-phenotype and functions of dendritic cells in an inhibitor-specific manner. Int Immunopharmacol 32:118-124.

Kaidanovich-Beilin O, Milman A, Weizman A, Pick CG, and Eldar-Finkelman H (2004) Rapid antidepressive-like activity of specific glycogen synthase kinase-3 inhibitor and its effect on beta-catenin in mouse hippocampus. Biol Psychiatry 55:781-784.

Kakugawa S, Langton PF, Zebisch M, Howell S, Chang TH, Liu Y, Feizi T, Bineva G, O'Reilly N, Snijders AP, et al. (2015) Notum deacylates Wnt proteins to suppress signalling activity. Nature 519:187-192.

Kasi PD, Tamilselvam R, Skalicka-Woźniak K, Nabavi SF, Daglia M, Bishayee A, Pazoki-Toroudi H, and Nabavi SM (2016) Molecular targets of curcumin for cancer therapy: an updated review. Tumour Biol 37:13017-13028.
Kato M, Patel MS, Levasseur R, Lobov I, Chang BH, Glass DA II, Hartmann C, Li L Hwang TH, Brayton CF, et al. (2002) Cbfa1-independent decrease in osteoblast proliferation, osteopenia, and persistent embryonic eye vascularization in mice deficient in Lrp5, a Wnt coreceptor. J Cell Biol 157:303-314.

Kazanskaya O, Ohkawara B, Heroult M, Wu W, Maltry N, Augustin HG, and Niehrs C (2008) The Wnt signaling regulator R-spondin 3 promotes angioblast and vascular development. Development 135:3655-3664.

Kerkela R, Kockeritz L, Macaulay K, Zhou J, Doble BW, Beahm C, Greytak S, Woulfe K, Trivedi CM, Woodgett JR, et al. (2008) Deletion of GSK-3beta in mice leads to hypertrophic cardiomyopathy secondary to cardiomyoblast hyperproliferation. $J$ Clin Invest 118:3609-3618.

Kerkelä R, Woulfe K, and Force T (2007) Glycogen synthase kinase-3beta - actively inhibiting hypertrophy. Trends Cardiovasc Med 17:91-96

Kibar Z, Torban E, McDearmid JR, Reynolds A, Berghout J, Mathieu M, Kirillova I, De Marco P, Merello E, Hayes JM, et al. (2007) Mutations in VANGL1 associated with neural-tube defects. N Engl J Med 356:1432-1437.

Kikuchi K and Poss KD (2012) Cardiac regenerative capacity and mechanisms. Annu Rev Cell Dev Biol 28:719-741.

Kim HM, Kim CS, Lee JH, Jang SJ, Hwang JJ, Ro S, and Choi J (2013) CG0009, a novel glycogen synthase kinase 3 inhibitor, induces cell death through cyclin D1 depletion in breast cancer cells. PLoS One 8:e60383.

Kim HY, Choi S, Yoon JH, Lim HJ, Lee H, Choi J, Ro EJ, Heo JN, Lee W, No KT, et al. (2016) Small molecule inhibitors of the dishevelled-CXXC5 interaction are new drug candidates for bone anabolic osteoporosis therapy. EMBO Mol Med 8: 375-387.

Kim HY, Yoon JY, Yun JH, Cho KW, Lee SH, Rhee YM, Jung HS, Lim HJ, Lee H, Choi J, et al. (2015) CXXC5 is a negative-feedback regulator of the Wnt/ $\beta$-catenin pathway involved in osteoblast differentiation. Cell Death Differ 22:912-920.

Kim J, Kim J, Kim DW, Ha Y, Ihm MH, Kim H, Song K, and Lee I (2010a) Wnt5a induces endothelial inflammation via beta-catenin-independent signaling. $J$ Immunol 185:1274-1282.

Kim SY, Dunn IF, Firestein R, Gupta P, Wardwell L, Repich K, Schinzel AC, Wittner B, Silver SJ, Root DE, et al. (2010b) CK1epsilon is required for breast cancers dependent on beta-catenin activity. PLoS One 5:e8979.

Kim YJ, Kim JT, Bae YC, Suh KT, and Jung JS (2008) ICAT participates in proliferation and osteogenic differentiation of human adipose tissue-derived mesenchymal stem cell. Life Sci 83:851-858.

Kim YR, Kim CS, Naqvi A, Kumar A, Kumar S, Hoffman TA, and Irani K (2012) Epigenetic upregulation of p66shc mediates low-density lipoprotein cholesterolinduced endothelial cell dysfunction. Am J Physiol Heart Circ Physiol 303: H189-H196

Kinzler KW and Vogelstein B (1996) Lessons from hereditary colorectal cancer. Cell 87:159-170.

Kirk JA, Holewinski RJ, Kooij V, Agnetti G, Tunin RS, Witayavanitkul N, de Tombe PP, Gao WD, Van Eyk J, and Kass DA (2014) Cardiac resynchronization sensitizes the sarcomere to calcium by reactivating GSK-3 $\beta$. J Clin Invest 124:129-138.

Kizil C, Otto GW, Geisler R, Nüsslein-Volhard C, and Antos CL (2009) Simplet controls cell proliferation and gene transcription during zebrafish caudal fin regeneration. Dev Biol 325:329-340.

Klaus A, Saga Y, Taketo MM, Tzahor E, and Birchmeier W (2007) Distinct roles of Wnt/beta-catenin and Bmp signaling during early cardiogenesis. Proc Natl Acad Sci USA 104:18531-18536.

Klein PS and Melton DA (1996) A molecular mechanism for the effect of lithium on development. Proc Natl Acad Sci USA 93:8455-8459.

Klingensmith J, Nusse R, and Perrimon N (1994) The Drosophila segment polarity gene dishevelled encodes a novel protein required for response to the wingless signal. Genes Dev 8:118-130.

Kobayashi K, Luo M, Zhang Y, Wilkes DC, Ge G, Grieskamp T, Yamada C, Liu TC, Huang G, Basson CT, et al. (2009) Secreted Frizzled-related protein 2 is a procollagen $\mathrm{C}$ proteinase enhancer with a role in fibrosis associated with myocardia infarction. Nat Cell Biol 11:46-55.

Kohler EE, Baruah J, Urao N, Ushio-Fukai M, Fukai T, Chatterjee I, and Wary KK (2014) Low-dose 6-bromoindirubin-3'-oxime induces partial dedifferentiation of endothelial cells to promote increased neovascularization. Stem Cells $\mathbf{3 2}$ 1538-1552.

Komekado H, Yamamoto H, Chiba T, and Kikuchi A (2007) Glycosylation and palmitoylation of Wnt-3a are coupled to produce an active form of Wnt-3a. Genes Cells 12:521-534. Koo BK, Spit M, Jordens I, Low TY, Stange DE, van de Wetering M, van Es JH, Mohammed S, Heck AJ, Maurice MM, et al. (2012) Tumour suppressor RNF43 is a stem-cell E3 ligase that induces endocytosis of Wnt receptors. Nature 488:665-669.

Koopmans T, Crutzen S, Menzen MH, Halayko AJ, Hackett TL, Knight DA, and Gosens R (2016) Selective targeting of CREB-binding protein/ $\beta$-catenin inhibits growth of and extracellular matrix remodelling by airway smooth muscle. $B r$ $J$ Pharmacol 173:3327-3341.

Korinek V, Barker N, Morin PJ, van Wichen D, de Weger R, Kinzler KW, Vogelstein $\mathrm{B}$, and Clevers H (1997) Constitutive transcriptional activation by a beta-cateninTcf complex in APC-/- colon carcinoma. Science 275:1784-1787.

Korn C, Scholz B, Hu J, Srivastava K, Wojtarowicz J, Arnsperger T, Adams RH, Boutros M, Augustin HG, and Augustin I (2014) Endothelial cell-derived noncanonical Wnt ligands control vascular pruning in angiogenesis. Development 141: 1757-1766

Koval A and Katanaev VL (2012) Platforms for high-throughput screening of Wnt/ Frizzled antagonists. Drug Discov Today 17:1316-1322.

Koyanagi M, Haendeler J, Badorff C, Brandes RP, Hoffmann J, Pandur P, Zeiher AM Kühl M, and Dimmeler S (2005) Non-canonical Wnt signaling enhances differentiation of human circulating progenitor cells to cardiomyogenic cells. J Biol Chem 280:16838-16842.

Krause U, Harris S, Green A, Ylostalo J, Zeitouni S, Lee N, and Gregory CA (2010) Pharmaceutical modulation of canonical Wnt signaling in multipotent stromal cells for improved osteoinductive therapy. Proc Natl Acad Sci USA 107:4147-4152. 
Krishna SM, Seto SW, Jose RJ, Li J, Morton SK, Biros E, Wang Y, Nsengiyumva V, Lindeman JH, Loots GG, et al. (2017) Wnt signaling pathway inhibitor sclerostin inhibits angiotensin II-induced aortic aneurysm and atherosclerosis. Arterioscler Thromb Vasc Biol 37:553-566.

Kumawat K, Koopmans T, Menzen MH, Prins A, Smit M, Halayko AJ, and Gosens R (2016) Cooperative signaling by TGF- $\beta 1$ and WNT-11 drives sm- $\alpha$-actin expression in smooth muscle via Rho kinase-actin-MRTF-A signaling. Am J Physiol Lung Cell Mol Physiol 311:L529-L537.

Kurayoshi M, Yamamoto H, Izumi S, and Kikuchi A (2007) Post-translational palmitoylation and glycosylation of Wnt-5a are necessary for its signalling. Biochem $J$ 402:515-523.

Kusserow A, Pang K, Sturm C, Hrouda M, Lentfer J, Schmidt HA, Technau U, von Haeseler A, Hobmayer B, Martindale MQ, et al. (2005) Unexpected complexity of the Wnt gene family in a sea anemone. Nature 433:156-160.

Kuwahara K and Nakao K (2011) New molecular mechanisms for cardiovascular disease:transcriptional pathways and novel therapeutic targets in heart failure. $J$ Pharmacol Sci 116:337-342.

Kwon C, Arnold J, Hsiao EC, Taketo MM, Conklin BR, and Srivastava D (2007) Canonical Wnt signaling is a positive regulator of mammalian cardiac progenitors. Proc Natl Acad Sci USA 104:10894-10899.

Laco F, Low JL, Seow J, Woo TL, Zhong Q, Seayad J, Liu Z, Wei H, Reuveny S, Elliott $\mathrm{DA}$, et al. (2015) Cardiomyocyte differentiation of pluripotent stem cells with SB203580 analogues correlates with Wnt pathway CK1 inhibition independent of p38 MAPK signaling. $J$ Mol Cell Cardiol 80:56-70.

Laeremans H, Hackeng TM, van Zandvoort MA, Thijssen VL, Janssen BJ, Ottenheijm HC, Smits JF, and Blankesteijn WM (2011) Blocking of frizzled signaling with a homologous peptide fragment of wnt3a/wnt5a reduces infarct expansion and prevents the development of heart failure after myocardial infarction. Circulation 124:1626-1635.

Laflamme MA and Murry CE (2011) Heart regeneration. Nature 473:326-335.

Lal H, Ahmad F, Woodgett J, and Force T (2015) The GSK-3 family as therapeutic target for myocardial diseases. Circ Res 116:138-149.

Lal H, Ahmad F, Zhou J, Yu JE, Vagnozzi RJ, Guo Y, Yu D, Tsai EJ, Woodgett J, Gao E, et al. (2014) Cardiac fibroblast glycogen synthase kinase-3ß regulates ventricular remodeling and dysfunction in ischemic heart. Circulation 130 419-430.

Lal H, Zhou J, Ahmad F, Zaka R, Vagnozzi RJ, Decaul M, Woodgett J, Gao E, and Force $\mathrm{T}$ (2012) Glycogen synthase kinase-3 $\alpha$ limits ischemic injury, cardiac rupture, post-myocardial infarction remodeling and death. Circulation 125:65-75

Landman EB, Miclea RL, van Blitterswijk CA, and Karperien M (2013) Small molecule inhibitors of WNT/ $\beta$-catenin signaling block IL- $1 \beta$ - and TNF $\alpha$-induced cartilage degradation. Arthritis Res Ther 15:R93.

Lange C, Mix E, Frahm J, Glass A, Müller J, Schmitt O, Schmöle AC, Klemm K, Ortinau S, Hübner R, et al. (2011) Small molecule GSK-3 inhibitors increase neurogenesis of human neural progenitor cells. Neurosci Lett 488:36-40.

Langton PF, Kakugawa S, and Vincent JP (2016) Making, exporting, and modulating Wnts. Trends Cell Biol 26:756-765.

Lau T, Chan E, Callow M, Waaler J, Boggs J, Blake RA, Magnuson S, Sambrone A Schutten M, Firestein R, et al. (2013) A novel tankyrase small-molecule inhibitor suppresses APC mutation-driven colorectal tumor growth. Cancer Res 73:3132-3144.

Laumanns IP, Fink L, Wilhelm J, Wolff JC, Mitnacht-Kraus R, Graef-Hoechst S, Stein MM, Bohle RM, Klepetko W, Hoda MA, et al. (2009) The noncanonical WNT pathway is operative in idiopathic pulmonary arterial hypertension. Am J Respir Cell Mol Biol 40:683-691.

Le PN, McDermott JD, and Jimeno A (2015) Targeting the Wnt pathway in human cancers: therapeutic targeting with a focus on OMP-54F28. Pharmacol Ther 146:1-11.

Le Dour C, Macquart C, Sera F, Homma S, Bonne G, Morrow JP, Worman HJ, and Muchir A (2017) Decreased WNT/ $\beta$-catenin signalling contributes to the pathogenesis of dilated cardiomyopathy caused by mutations in the lamin $\mathrm{a} / \mathrm{C}$ gene. Hum Mol Genet 26:333-343.

Leal LF, Bueno AC, Gomes DC, Abduch R, de Castro M, and Antonini SR (2015) Inhibition of the Tcf/beta-catenin complex increases apoptosis and impairs adrenocortical tumor cell proliferation and adrenal steroidogenesis. Oncotarget 6: 43016-43032.

Lee HJ, Bao J, Miller A, Zhang C, Wu J, Baday YC, Guibao C, Li L, Wu D, and Zheng JJ (2015) Structure-based discovery of novel small molecule Wnt signaling inhibitors by targeting the cysteine-rich domain of frizzled. J Biol Chem 290: 30596-30606.

Lee HJ, Wang NX, Shi DL, and Zheng JJ (2009) Sulindac inhibits canonical Wnt signaling by blocking the PDZ domain of the protein dishevelled. Angew Chem Int Ed Engl 48:6448-6452.

Lee SY, Lim TG, Chen H, Jung SK, Lee HJ, Lee MH, Kim DJ, Shin A, Lee KW, Bode AM, et al. (2013) Esculetin suppresses proliferation of human colon cancer cells by directly targeting $\beta$-catenin. Cancer Prev Res (Phila) 6:1356-1364.

Lehtiö L, Chi NW, and Krauss S (2013) Tankyrases as drug targets. FEBS J 280 3576-3593.

Lepourcelet M, Chen YN, France DS, Wang H, Crews P, Petersen F, Bruseo C, Wood AW, and Shivdasani RA (2004) Small-molecule antagonists of the oncogenic Tcf/ beta-catenin protein complex. Cancer Cell 5:91-102.

Li H, Jiao S, Li X, Banu H, Hamal S, and Wang X (2015) Therapeutic effects of antibiotic drug tigecycline against cervical squamous cell carcinoma by inhibiting Wnt/ß-catenin signaling. Biochem Biophys Res Commun 467:14-20.

Li J, Chen X, Ding X, Cheng Y, Zhao B, Lai ZC, Al Hezaimi K, Hakem R, Guan KL, and Wang CY (2013a) LATS2 suppresses oncogenic Wnt signaling by disrupting $\beta$-catenin/BCL9 interaction. Cell Reports 5:1650-1663.

Li M, Hu X, Zhu J, Zhu C, Zhu S, Liu X, Xu J, Han S, and Yu Z (2014a) Overexpression of miR-19b impairs cardiac development in zebrafish by targeting ctnnb1. Cell Physiol Biochem 33:1988-2002.

Li N, Xi Y, Tinsley HN, Gurpinar E, Gary BD, Zhu B, Li Y, Chen X, Keeton AB, Abadi $\mathrm{AH}$, et al. (2013b) Sulindac selectively inhibits colon tumor cell growth by activating the cGMP/PKG pathway to suppress Wnt/ $\beta$-catenin signaling. Mol Cancer Ther 12:1848-1859.

Li R, Beebe T, Jen N, Yu F, Takabe W, Harrison M, Cao H, Lee J, Yang H, Han P, et al. (2014b) Shear stress-activated Wnt-angiopoietin-2 signaling recapitulates vascular repair in zebrafish embryos. Arterioscler Thromb Vasc Biol 34: $2268-2275$

Li X, Pu J, Jiang S, Su J, Kong L, Mao B, Sun H, and Li Y (2013c) Henryin, an entkaurane diterpenoid, inhibits Wnt signaling through interference with $\beta$-catenin/ TCF4 interaction in colorectal cancer cells. PLoS One 8:e68525

Li X, Zhang Y, Kang H, Liu W, Liu P, Zhang J, Harris SE, and Wu D (2005) Sclerostin binds to LRP5/6 and antagonizes canonical Wnt signaling. $J$ Biol Chem 280: 19883-19887.

Li Y, Li PK, Roberts MJ, Arend RC, Samant RS, and Buchsbaum DJ (2014c) Multitargeted therapy of cancer by niclosamide: a new application for an old drug. Cancer Lett 349:8-14.

Liang MH and Chuang DM (2007) Regulation and function of glycogen synthase kinase-3 isoforms in neuronal survival. J Biol Chem 282:3904-3917.

Liang Q and Molkentin JD (2002) Divergent signaling pathways converge on GATA4 to regulate cardiac hypertrophic gene expression. J Mol Cell Cardiol 34:611-616. Libby P (2002) Inflammation in atherosclerosis. Nature 420:868-874.

Lickert H, Kutsch S, Kanzler B, Tamai Y, Taketo MM, and Kemler R (2002) Formation of multiple hearts in mice following deletion of beta-catenin in the embryonic endoderm. Dev Cell 3:171-181.

Liebner S, Cattelino A, Gallini R, Rudini N, Iurlaro M, Piccolo S, and Dejana E (2004) Beta-catenin is required for endothelial-mesenchymal transformation during heart cushion development in the mouse. J Cell Biol 166:359-367.

Liebner S, Corada M, Bangsow T, Babbage J, Taddei A, Czupalla CJ, Reis M, Felici A, Wolburg H, Fruttiger M, et al. (2008) Wnt/beta-catenin signaling controls development of the blood-brain barrier. J Cell Biol 183:409-417.

Lieu C, Heymach J, Overman M, Tran H, and Kopetz S (2011) Beyond VEGF: inhibition of the fibroblast growth factor pathway and antiangiogenesis. Clin Cancer Res 17:6130-6139.

Lijam N, Paylor R, McDonald MP, Crawley JN, Deng CX, Herrup K, Stevens KE, Maccaferri G, McBain C.J, Sussman DJ, et al. (1997) Social interaction and sensorimotor gating abnormalities in mice lacking Dvl1. Cell 90:895-905.

Lin H, Angeli M, Chung KJ, Ejimadu C, Rosa AR, and Lee T (2016) sFRP2 activates Wnt/ $\beta$-catenin signaling in cardiac fibroblasts: differential roles in cell growth energy metabolism, and extracellular matrix remodeling. Am J Physiol Cell Physiol 311:C710-C719.

Liu J, Pan S, Hsieh MH, Ng N, Sun F, Wang T, Kasibhatla S, Schuller AG, Li AG, Cheng D, et al. (2013) Targeting Wnt-driven cancer through the inhibition of Porcupine by LGK974. Proc Natl Acad Sci USA 110:20224-20229.

Liu P, Wakamiya M, Shea MJ, Albrecht U, Behringer RR, and Bradley A (1999) Requirement for Wnt3 in vertebrate axis formation. Nat Genet 22:361-365.

Liu X and Xu Z (2016) Osteogenesis in calcified aortic valve disease: from histopathological observation towards molecular understanding. Prog Biophys Mol Biol 122:156-161.

Liu Y, Wang WM, Zhang XL, He HQ, Sun XL, Zeng H, Xu XF, Huang L, Zhu Z, Zhang $\mathrm{L}$, et al. (2016) AGE/RAGE promotes thecalcification of human aortic smooth muscle cells via the Wnt/ $\beta$-catenin axis. Am J Transl Res 8:4644-4656.

Liu YC, Zou XB, Chai YF, and Yao YM (2014) Macrophage polarization in inflammatory diseases. Int J Biol Sci 10:520-529.

Lo CW (2000) Role of gap junctions in cardiac conduction and development: insights from the connexin knockout mice. Circ Res 87:346-348.

Loh KM, van Amerongen R, and Nusse R (2016) Generating cellular diversity and spatial form: Wnt signaling and the evolution of multicellular animals. Dev Cell 38: $643-655$

Lombardi R, Dong J, Rodriguez G, Bell A, Leung TK, Schwartz RJ, Willerson JT, Brugada R, and Marian AJ (2009) Genetic fate mapping identifies second heart field progenitor cells as a source of adipocytes in arrhythmogenic right ventricular cardiomyopathy. Circ Res 104:1076-1084.

Lorenowicz MJ and Korswagen HC (2009) Sailing with the Wnt: charting the Wnt processing and secretion route. Exp Cell Res 315:2683-2689.

Lu D, Bao D, Dong W, Liu N, Zhang X, Gao S, Ge W, Gao X, and Zhang L (2016) Dkk3 prevents familial dilated cardiomyopathy development through Wnt pathway. Lab Invest 96:239-248.

Lu D, Choi MY, Yu J, Castro JE, Kipps TJ, and Carson DA (2011a) Salinomycin inhibits Wnt signaling and selectively induces apoptosis in chronic lymphocytic leukemia cells. Proc Natl Acad Sci USA 108:13253-13257.

$\mathrm{Lu} \mathrm{W}$ and Li Y (2014) Salinomycin suppresses LRP6 expression and inhibits both Wnt/ $\beta$-catenin and mTORC1 signaling in breast and prostate cancer cells. J Cell Biochem 115:1799-1807.

Lu W, Lin C, King TD, Chen H, Reynolds RC, and Li Y (2012) Silibinin inhibits Wnt/ $\beta$-catenin signaling by suppressing Wnt co-receptor LRP6 expression in human prostate and breast cancer cells. Cell Signal 24:2291-2296.

Lu W, Lin C, Roberts MJ, Waud WR, Piazza GA, and Li Y (2011b) Niclosamide suppresses cancer cell growth by inducing Wnt co-receptor LRP6 degradation and inhibiting the Wnt/ $\beta$-catenin pathway. PLoS One 6:e29290.

Luhmann UF, Meunier D, Shi W, Lüttges A, Pfarrer C, Fundele R, and Berger W (2005) Fetal loss in homozygous mutant Norrie disease mice: a new role of Norrin in reproduction. Genesis 42:253-262.

Lund SA, Giachelli CM, and Scatena M (2009) The role of osteopontin in inflammatory processes. J Cell Commun Signal 3:311-322.

Lusis AJ (2000) Atherosclerosis. Nature 407:233-241.

Lustig B, Jerchow B, Sachs M, Weiler S, Pietsch T, Karsten U, van de Wetering M, Clevers H, Schlag PM, Birchmeier W, et al. (2002) Negative feedback loop of Wnt signaling through upregulation of conductin/axin2 in colorectal and liver tumors. Mol Cell Biol 22:1184-1193.

Ma L and Wang HY (2007) Mitogen-activated protein kinase p38 regulates the Wnt/ cyclic GMP/Ca2+ non-canonical pathway. J Biol Chem 282:28980-28990. 
Ma L, Wang X, Jia T, Wei W, Chua MS, and So S (2015) Tankyrase inhibitors attenuate WNT/ $\beta$-catenin signaling and inhibit growth of hepatocellular carcinoma cells. Oncotarget 6:25390-25401.

MacDonald BT and He X (2012) Frizzled and LRP5/6 receptors for Wnt/ $\beta$-catenin signaling. Cold Spring Harb Perspect Biol 4:a007880.

MacDonald BT, Tamai K, and He X (2009) Wnt/beta-catenin signaling: components, mechanisms, and diseases. Dev Cell 17:9-26.

Madan B, Ke Z, Harmston N, Ho SY, Frois AO, Alam J, Jeyaraj DA, Pendharkar V, Ghosh K, Virshup IH, et al. (2016a) Wnt addiction of genetically defined cancers reversed by PORCN inhibition. Oncogene 35:2197-2207.

Madan B, Patel MB, Zhang J, Bunte RM, Rudemiller NP, Griffiths R, Virshup DM, and Crowley SD (2016b) Experimental inhibition of porcupine-mediated Wnt O-acylation attenuates kidney fibrosis. Kidney Int 89:1062-1074.

Magoori K, Kang MJ, Ito MR, Kakuuchi H, Ioka RX, Kamataki A, Kim DH, Asaba H Iwasaki S, Takei YA, et al. (2003) Severe hypercholesterolemia, impaired fat tolerance, and advanced atherosclerosis in mice lacking both low density lipoprotein receptor-related protein 5 and apolipoprotein E. J Biol Chem 278:11331-11336.

Malekar P, Hagenmueller M, Anyanwu A, Buss S, Streit MR, Weiss CS, Wolf D, Riffel J, Bauer A, Katus HA, et al. (2010) Wnt signaling is critical for maladaptive cardiac hypertrophy and accelerates myocardial remodeling. Hypertension 55: 939-945

Malgor R, Bhatt PM, Connolly BA, Jacoby DL, Feldmann KJ, Silver MJ, Nakazawa M, McCall KD, and Goetz DJ (2014) Wnt5a, TLR2 and TLR4 are elevated in advanced human atherosclerotic lesions. Inflamm Res 63:277-285.

Mani A, Radhakrishnan J, Wang H, Mani A, Mani MA, Nelson-Williams C, Carew KS, Mane S, Najmabadi H, Wu D, et al. (2007) LRP6 mutation in a family with early coronary disease and metabolic risk factors. Science 315:1278-1282.

Mao B, Wu W, Davidson G, Marhold J, Li M, Mechler BM, Delius H, Hoppe D, Stannek P, Walter C, et al. (2002) Kremen proteins are Dickkopf receptors that regulate Wnt/beta-catenin signalling. Nature 417:664-667.

Mao B, Wu W, Li Y, Hoppe D, Stannek P, Glinka A, and Niehrs C (2001) LDLreceptor-related protein 6 is a receptor for Dickkopf proteins. Nature 411:321-325.

Marchand A, Atassi F, Gaaya A, Leprince P, Le Feuvre C, Soubrier F, Lompré AM, and Nadaud S (2011) The Wnt/beta-catenin pathway is activated during advanced arterial aging in humans. Aging Cell 10:220-232.

Martin-Padura I, de Nigris F, Migliaccio E, Mansueto G, Minardi S, Rienzo M, Lerman LO, Stendardo M, Giorgio M, De Rosa G, et al. (2008) p66Shc deletion confers vascular protection in advanced atherosclerosis in hypercholesterolemic apolipoprotein E knockout mice. Endothelium 15:276-287.

Marvin MJ, Di Rocco G, Gardiner A, Bush SM, and Lassar AB (2001) Inhibition of Wnt activity induces heart formation from posterior mesoderm. Genes Dev $\mathbf{1 5}$ 316-327.

Masckauchán TN, Agalliu D, Vorontchikhina M, Ahn A, Parmalee NL, Li CM, Khoo A, Tycko B, Brown AM, and Kitajewski J (2006) Wnt5a signaling induces proliferation and survival of endothelial cells in vitro and expression of MMP-1 and Tie-2. Mol Biol Cell 17:5163-5172.

Mastri M, Shah Z, Hsieh K, Wang X, Wooldridge B, Martin S, Suzuki G, and Lee T (2014) Secreted frizzled-related protein 2 as a target in antifibrotic therapeutic intervention. Am J Physiol Cell Physiol 306:C531-C539.

Masuelli L, Bei R, Sacchetti P, Scappaticci I, Francalanci P, Albonici L, Coletti A Palumbo C, Minieri M, Fiaccavento R, et al. (2003) Beta-catenin accumulates in intercalated disks of hypertrophic cardiomyopathic hearts. Cardiovasc Res 60 : $376-387$.

Mathieu P, Boulanger MC, and Bouchareb R (2014) Molecular biology of calcific aortic valve disease: towards new pharmacological therapies. Expert Rev Cardiovasc Ther 12:851-862.

Matsuda T, Zhai P, Maejima Y, Hong C, Gao S, Tian B, Goto K, Takagi H, TamamoriAdachi M, Kitajima S, et al. (2008) Distinct roles of GSK-3alpha and GSK-3beta phosphorylation in the heart under pressure overload. Proc Natl Acad Sci USA 105:20900-20905.

Matsukawa Y, Nishino H, Okuyama Y, Matsui T, Matsumoto T, Matsumura S, Shimizu Y, Sowa Y, and Sakai T (1997) Effects of quercetin and/or restraint stress on formation of aberrant crypt foci induced by azoxymethane in rat colons. Oncology 54:118-121.

Matteucci M, Casieri V, Gabisonia K, Aquaro GD, Agostini S, Pollio G, Diamanti D, Rossi M, Travagli M, Porcari V, et al. (2016) Magnetic resonance imaging of infarctinduced canonical wingless/integrated (Wnt)/ $\beta$-catenin/T-cell factor pathway activation, in vivo. Cardiovasc Res 112:645-655.

Matthijs Blankesteijn W and Hermans KC (2015) Wnt signaling in atherosclerosis. Eur J Pharmacol 763 (Pt A):122-130.

McCrea PD and Gottardi CJ (2016) Beyond $\beta$-catenin: prospects for a larger catenin network in the nucleus. Nat Rev Mol Cell Biol 17:55-64.

McGonigle S, Chen Z, Wu J, Chang P, Kolber-Simonds D, Ackermann K, Twine NC, Shie JL, Miu JT, Huang KC, et al. (2015) E7449: a dual inhibitor of PARP1/2 and tankyrase $1 / 2$ inhibits growth of DNA repair deficient tumors and antagonizes Wnt signaling. Oncotarget 6:41307-41323.

Michael A, Haq S, Chen X, Hsich E, Cui L, Walters B, Shao Z, Bhattacharya K, Kilter $\mathrm{H}$, Huggins G, et al. (2004) Glycogen synthase kinase-3beta regulates growth, calcium homeostasis, and diastolic function in the heart. $J$ Biol Chem 279: 21383-21393.

Migliaccio E, Giorgio M, Mele S, Pelicci G, Reboldi P, Pandolfi PP, Lanfrancone L, and Pelicci PG (1999) The p66shc adaptor protein controls oxidative stress response and life span in mammals. Nature 402:309-313.

Mill C and George SJ (2012) Wnt signalling in smooth muscle cells and its role in cardiovascular disorders. Cardiovasc Res 95:233-240.

Milligan G (2009) G protein-coupled receptor hetero-dimerization: contribution to pharmacology and function. Br J Pharmacol 158:5-14.

Min JK, Park H, Choi HJ, Kim Y, Pyun BJ, Agrawal V, Song BW, Jeon J, Maeng YS, Rho SS, et al. (2011) The WNT antagonist Dickkopf2 promotes angiogenesis in rodent and human endothelial cells. J Clin Invest 121:1882-1893.
Mirotsou M, Zhang Z, Deb A, Zhang L, Gnecchi M, Noiseux N, Mu H, Pachori A and Dzau V (2007) Secreted frizzled related protein 2 (Sfrp2) is the key Aktmesenchymal stem cell-released paracrine factor mediating myocardial survival and repair. Proc Natl Acad Sci USA 104:1643-1648.

Miyabayashi T, Teo JL, Yamamoto M, McMillan M, Nguyen C, and Kahn M (2007) Wnt/beta-catenin/CBP signaling maintains long-term murine embryonic stem cell pluripotency. Proc Natl Acad Sci USA 104:5668-5673.

Mizutani M, Wu JC, and Nusse R (2016) Fibrosis of the neonatal mouse heart after cryoinjury is accompanied by Wnt signaling activation and epicardial-tomesenchymal transition. J Am Heart Assoc 5:e002457.

Mlodzik M (2016) The dishevelled protein family: still rather a mystery after over 20 years of molecular studies. Curr Top Dev Biol 117:75-91.

Mohamed TM, Abou-Leisa R, Stafford N, Maqsood A, Zi M, Prehar S, BaudoinStanley F, Wang X, Neyses L, Cartwright EJ, et al. (2016) The plasma membrane calcium ATPase 4 signalling in cardiac fibroblasts mediates cardiomyocyte hypertrophy. Nat Commun 7:11074.

Molenaar M, van de Wetering M, Oosterwegel M, Peterson-Maduro J, Godsave S, Korinek V, Roose J, Destrée O, and Clevers H (1996) XTcf-3 transcription factor mediates beta-catenin-induced axis formation in Xenopus embryos. Cell 86 391-399.

Moon J, Zhou H, Zhang LS, Tan W, Liu Y, Zhang S, Morlock LK, Bao X, Palecek SP Feng JQ, et al. (2017) Blockade to pathological remodeling of infarcted heart tissue using a porcupine antagonist. Proc Natl Acad Sci USA 114:1649-1654.

Moore WJ, Kern JC, Bhat R, Bodine PV, Fukyama S, Krishnamurthy G, Magolda RL, Pitts K, Stauffer B, and Trybulski EJ (2010) Modulation of Wnt signaling through inhibition of secreted frizzled-related protein I (sFRP-1) with N-substituted piperidinyl diphenylsulfonyl sulfonamides: part II. Bioorg Med Chem 18:190-201.

Moorman AF and Christoffels VM (2003) Cardiac chamber formation: development, genes, and evolution. Physiol Rev 83:1223-1267.

Morini MF and Dejana E (2014) Transcriptional regulation of arterial differentiation via Wnt, Sox and Notch. Curr Opin Hematol 21:229-234

Morisco C, Zebrowski D, Condorelli G, Tsichlis P, Vatner SF, and Sadoshima J (2000) The Akt-glycogen synthase kinase 3beta pathway regulates transcription of atrial natriuretic factor induced by beta-adrenergic receptor stimulation in cardiac myocytes. J Biol Chem 275:14466-14475.

Morishita Y, Kobayashi K, Klyachko E, Jujo K, Maeda K, Losordo DW, and Murohara T (2016) Wnt11 gene therapy with adeno-associated virus 9 improves recovery from myocardial infarction by modulating the inflammatory response. Sci Rep 6:21705.

Motiwala SR, Szymonifka J, Belcher A, Weiner RB, Baggish AL, Gaggin HK, Bhardwaj A, and Januzzi JL Jr (2014) Measurement of novel biomarkers to predict chronic heart failure outcomes and left ventricular remodeling. J Cardiovasc Transl Res 7:250-261.

Motovska Z, Vichova T, Doktorova M, Labos M, Maly M, and Widimsky P (2015) Serum Dickkopf-1 signaling and calcium deposition in aortic valve are significantly related to the presence of concomitant coronary atherosclerosis in patients with symptomatic calcified aortic stenosis. J Transl Med 13:63.

Mottet D, Dumont V, Deccache Y, Demazy C, Ninane N, Raes M, and Michiels C (2003) Regulation of hypoxia-inducible factor-1alpha protein level during hypoxic conditions by the phosphatidylinositol 3-kinase/Akt/glycogen synthase kinase 3beta pathway in HepG2 cells. J Biol Chem 278:31277-31285.

Mulligan KA, Fuerer C, Ching W, Fish M, Willert K, and Nusse R (2012) Secreted Wingless-interacting molecule (Swim) promotes long-range signaling by maintaining Wingless solubility. Proc Natl Acad Sci USA 109:370-377.

Murakoshi M, Saiki K, Urayama K, and Sato TN (2013) An anthelmintic drug, pyrvinium pamoate, thwarts fibrosis and ameliorates myocardial contractile dysfunction in a mouse model of myocardial infarction. PLoS One 8:e79374.

Murray PJ and Wynn TA (2011) Protective and pathogenic functions of macrophage subsets. Nat Rev Immunol 11:723-737.

Nagayama S, Fukukawa C, Katagiri T, Okamoto T, Aoyama T, Oyaizu N, Imamura M, Toguchida J, and Nakamura Y (2005) Therapeutic potential of antibodies against FZD 10, a cell-surface protein, for synovial sarcomas. Oncogene 24 $6201-6212$

Naik S, Dothager RS, Marasa J, Lewis CL, and Piwnica-Worms D (2009) Vascular endothelial growth factor receptor-1 is synthetic lethal to aberrant beta-catenin activation in colon cancer. Clin Cancer Res 15:7529-7537.

Naito AT, Shiojima I, Akazawa H, Hidaka K, Morisaki T, Kikuchi A, and Komuro I (2006) Developmental stage-specific biphasic roles of Wnt/beta-catenin signaling in cardiomyogenesis and hematopoiesis. Proc Natl Acad Sci USA 103:19812-19817.

Nakagawa A, Naito AT, Sumida T, Nomura S, Shibamoto M, Higo T, Okada K, Sakai T, Hashimoto A, Kuramoto Y, et al. (2016) Activation of endothelial $\beta$-catenin signaling induces heart failure. Sci Rep 6:25009.

Nakamura K, Sano S, Fuster JJ, Kikuchi R, Shimizu I, Ohshima K, Katanasaka Y, Ouchi N, and Walsh K (2016) Secreted frizzled-related protein 5 diminishes cardiac inflammation and protects the heart from ischemia/reperfusion injury. J Biol Chem 291:2566-2575.

Nakamura T, Sano M, Songyang Z, and Schneider MD (2003) A Wnt- and beta -catenin-dependent pathway for mammalian cardiac myogenesis. Proc Natl Acad Sci USA 100:5834-5839.

Nakashima T, Ohkusa T, Okamoto Y, Yoshida M, Lee JK, Mizukami Y, and Yano M (2014) Rapid electrical stimulation causes alterations in cardiac intercellular junction proteins of cardiomyocytes. Am J Physiol Heart Circ Physiol 306: H1324-H1333.

Napoli C, Martin-Padura I, de Nigris F, Giorgio M, Mansueto G, Somma P, Condorelli M, Sica G, De Rosa G, and Pelicci P (2003) Deletion of the p66Shc longevity gene reduces systemic and tissue oxidative stress, vascular cell apoptosis, and early atherogenesis in mice fed a high-fat diet. Proc Natl Acad Sci USA 100 $2112-2116$

Narayanan BA, Doudican NA, Park J, Xu D, Narayanan NK, Dasgupta R, and Mazumder A (2012) Antagonistic effect of small-molecule inhibitors of Wnt/ $\beta$-catenin in multiple myeloma. Anticancer Res 32:4697-4707. 
Nathubhai A, Haikarainen T, Koivunen J, Murthy S, Koumanov F, Lloyd MD, Holman GD, Pihlajaniemi T, Tosh D, Lehtiö L, et al. (2017) Highly potent and isoform selective dual site binding Tankyrase/Wnt signaling inhibitors that increase cellular glucose uptake and have antiproliferative activity. $J$ Med Chem 60:814-820.

Naujok O, Lentes J, Diekmann U, Davenport C, and Lenzen S (2014) Cytotoxicity and activation of the Wnt/beta-catenin pathway in mouse embryonic stem cells treated with four GSK3 inhibitors. BMC Res Notes 7:273.

Ngo D, Sinha S, Shen D, Kuhn EW, Keyes MJ, Shi X, Benson MD, O’Sullivan JF, Keshishian H, Farrell LA, et al. (2016) Aptamer-based proteomic profiling reveals novel candidate biomarkers and pathways in cardiovascular disease. Circulation 134:270-285.

Nguyen G (2011) Renin, (pro)renin and receptor: an update. Clin Sci (Lond) 120 $169-178$.

Niehrs C and Shen J (2010) Regulation of Lrp6 phosphorylation. Cell Mol Life Sci 67: $2551-2562$.

Noda M, Vallon M, and Kuo CJ (2016) The Wnt7's tale: a story of an orphan who finds her tie to a famous family. Cancer Sci 107:576-582.

Nomi M, Oishi I, Kani S, Suzuki H, Matsuda T, Yoda A, Kitamura M, Itoh K, Takeuchi S, Takeda K, et al. (2001) Loss of mRor1 enhances the heart and skeletal abnormalities in mRor2-deficient mice: redundant and pleiotropic functions of mRor1 and mRor2 receptor tyrosine kinases. Mol Cell Biol 21:8329-8335.

Noordermeer J, Klingensmith J, Perrimon N, and Nusse R (1994) dishevelled and armadillo act in the wingless signalling pathway in Drosophila. Nature 367:80-83.

Norden J and Kispert A (2012) Wnt/Ctnnb1 signaling and the mesenchymal precursor pools of the heart. Trends Cardiovasc Med 22:118-122.

Nusse R and Varmus H (2012) Three decades of Wnts: a personal perspective on how a scientific field developed. EMBO J 31:2670-2684.

Nusse R and Varmus HE (1982) Many tumors induced by the mouse mammary tumor virus contain a provirus integrated in the same region of the host genome. Cell 31:99-109.

Nüsslein-Volhard C and Wieschaus E (1980) Mutations affecting segment number and polarity in Drosophila. Nature 287:795-801.

Oerlemans MI, Goumans MJ, van Middelaar B, Clevers H, Doevendans PA, and Sluijter JP (2010) Active Wnt signaling in response to cardiac injury. Basic Res Cardiol 105:631-641.

Ohishi K, Toume K, Arai MA, Koyano T, Kowithayakorn T, Mizoguchi T, Itoh M, and Ishibashi M (2015) 9-Hydroxycanthin-6-one, a $\beta$-carboline alkaloid from Eurycoma longifolia, is the first Wnt signal inhibitor through activation of glycogen synthase kinase $3 \beta$ without depending on casein kinase $1 \alpha . J$ Nat Prod 78 $1139-1146$.

Ohlmann A and Tamm ER (2012) Norrin: molecular and functional properties of an angiogenic and neuroprotective growth factor. Prog Retin Eye Res 31:243-257.

Oikonomopoulos A, Sereti KI, Conyers F, Bauer M, Liao A, Guan J, Crapps D, Han JK, Dong H, Bayomy AF, et al. (2011) Wnt signaling exerts an antiproliferative effect on adult cardiac progenitor cells through IGFBP3. Circ Res 109:1363-1374.

Oishi I, Suzuki H, Onishi N, Takada R, Kani S, Ohkawara B, Koshida I, Suzuki K, Yamada G, Schwabe GC, et al. (2003) The receptor tyrosine kinase Ror2 is involved in non-canonical Wnt5a/JNK signalling pathway. Genes Cells 8:645-654.

Ono M, Yin P, Navarro A, Moravek MB, Coon V JS, Druschitz SA, Gottardi CJ, and Bulun SE (2014) Inhibition of canonical WNT signaling attenuates human leiomyoma cell growth. Fertil Steril 101:1441-1449.

Orton EC, Lacerda CM, and MacLea HB (2012) Signaling pathways in mitral valve degeneration. $J$ Vet Cardiol 14:7-17.

Osada T, Chen M, Yang XY, Spasojevic I, Vandeusen JB, Hsu D, Clary BM, Clay TM Chen W, Morse MA, et al. (2011) Antihelminth compound niclosamide downregulates Wnt signaling and elicits antitumor responses in tumors with activating APC mutations. Cancer Res 71:4172-4182.

Osawa Y, Oboki K, Imamura J, Kojika E, Hayashi Y, Hishima T, Saibara T, Shibasaki F, Kohara M, and Kimura K (2015) Inhibition of cyclic adenosine monophosphate (cAMP)-response element-binding protein (CREB)-binding protein $(\mathrm{CBP}) / \beta$-catenin reduces liver fibrosis in mice. EBioMedicine 2:1751-1758.

Owens GK, Kumar MS, and Wamhoff BR (2004) Molecular regulation of vascular smooth muscle cell differentiation in development and disease. Physiol Rev 84 767-801.

Paes KT, Wang E, Henze K, Vogel P, Read R, Suwanichkul A, Kirkpatrick LL, Potter D, Newhouse MM, and Rice DS (2011) Frizzled 4 is required for retinal angiogenesis and maintenance of the blood-retina barrier. Invest Ophthalmol Vis Sci $\mathbf{5 2}$ 6452-6461.

Pahnke A, Conant G, Huyer LD, Zhao Y, Feric N, and Radisic M (2016) The role of Wnt regulation in heart development, cardiac repair and disease: a tissue engineering perspective. Biochem Biophys Res Commun 473:698-703.

Paik DT, Rai M, Ryzhov S, Sanders LN, Aisagbonhi O, Funke MJ, Feoktistov I, and Hatzopoulos AK (2015) Wnt10b gain-of-function improves cardiac repair by arteriole formation and attenuation of fibrosis. Circ Res 117:804-816.

Palevski D, Levin-Kotler LP, Kain D, Naftali-Shani N, Landa N, Ben-Mordechai T, Konfino T, Holbova R, Molotski N, Rosin-Arbesfeld R, et al. (2017) Loss of macrophage Wnt secretion improves remodeling and function after myocardial infarction in mice. J Am Heart Assoc 6:e04387.

Panáková D, Sprong H, Marois E, Thiele C, and Eaton S (2005) Lipoprotein particles are required for Hedgehog and Wingless signalling. Nature 435:58-65.

Pandur P, Läsche M, Eisenberg LM, and Kühl M (2002) Wnt-11 activation of a noncanonical Wnt signalling pathway is required for cardiogenesis. Nature 418: 636-641.

Park CH, Chang JY, Hahm ER, Park S, Kim HK, and Yang CH (2005) Quercetin, a potent inhibitor against beta-catenin/Tcf signaling in SW480 colon cancer cells. Biochem Biophys Res Commun 328:227-234.

Park M, Wu X, Golden K, Axelrod JD, and Bodmer R (1996) The wingless signaling pathway is directly involved in Drosophila heart development. Dev Biol 177: 104-116.
Patel DM and Green KJ (2014) Desmosomes in the heart: a review of clinical and mechanistic analyses. Cell Commun Adhes 21:109-128.

Peng Y, Zhang X, Feng X, Fan X, and Jin Z (2017) The crosstalk between microRNAs and the Wnt/ $\beta$-catenin signaling pathway in cancer. Oncotarget 8:14089-14106.

Peters JM, McKay RM, McKay JP, and Graff JM (1999) Casein kinase I transduces Wnt signals. Nature 401:345-350.

Phillips HM, Murdoch JN, Chaudhry B, Copp AJ, and Henderson DJ (2005) Vangl2 acts via RhoA signaling to regulate polarized cell movements during development of the proximal outflow tract. Circ Res 96:292-299.

Phillips MD, Mukhopadhyay M, Poscablo C, and Westphal H (2011) Dkk1 and Dkk2 regulate epicardial specification during mouse heart development. Int $J$ Cardiol 150:186-192.

Phng LK, Potente M, Leslie JD, Babbage J, Nyqvist D, Lobov I, Ondr JK, Rao S, Lang RA, Thurston G, et al. (2009) Nrarp coordinates endothelial Notch and Wnt signaling to control vessel density in angiogenesis. Dev Cell 16:70-82.

Phukan S, Babu VS, Kannoji A, Hariharan R, and Balaji VN (2010) GSK3beta: role in therapeutic landscape and development of modulators. Br J Pharmacol 160:1-19. Piersma B, Bank RA, and Boersema M (2015) Signaling in fibrosis: TGF- $\beta$, WNT, and YAP/TAZ Converge. Front Med (Lausanne) 2:59.

Pilichou K, Thiene G, Bauce B, Rigato I, Lazzarini E, Migliore F, Perazzolo Marra M, Rizzo S, Zorzi A, Daliento L, et al. (2016) Arrhythmogenic cardiomyopathy. Orphanet J Rare Dis 11:33.

Pinson KI, Brennan J, Monkley S, Avery BJ, and Skarnes WC (2000) An LDLreceptor-related protein mediates Wnt signalling in mice. Nature 407:535-538.

Plotkin B, Kaidanovich O, Talior I, and Eldar-Finkelman H (2003) Insulin mimetic action of synthetic phosphorylated peptide inhibitors of glycogen synthase kinase3. J Pharmacol Exp Ther 305:974-980.

Pode-Shakked N, Harari-Steinberg O, Haberman-Ziv Y, Rom-Gross E, Bahar S, Omer D, Metsuyanim S, Buzhor E, Jacob-Hirsch J, Goldstein RS, et al. (2011) Resistance or sensitivity of Wilms' tumor to anti-FZD7 antibody highlights the Wnt pathway as a possible therapeutic target. Oncogene 30:1664-1680.

Pongracz JE, Parnell SM, Jones T, Anderson G, and Jenkinson EJ (2006) Overexpression of ICAT highlights a role for catenin-mediated canonical Wnt signalling in early T cell development. Eur J Immunol 36:2376-2383.

Porrello ER and Olson EN (2014) A neonatal blueprint for cardiac regeneration. Stem Cell Res (Amst) 13 (3 Pt B):556-570.

Poss KD, Wilson LG, and Keating MT (2002) Heart regeneration in zebrafish. Science 298:2188-2190.

Proffitt KD, Madan B, Ke Z, Pendharkar V, Ding L, Lee MA, Hannoush RN, and Virshup DM (2013) Pharmacological inhibition of the Wnt acyltransferase PORCN prevents growth of WNT-driven mammary cancer. Cancer Res 73: 502-507.

Qian J, Zheng Y, Zheng C, Wang L, Qin H, Hong S, Li H, Lu Y, He J, Yang J, et al. (2012) Active vaccination with Dickkopf-1 induces protective and therapeutic antitumor immunity in murine multiple myeloma. Blood 119:161-169.

Qin L, Hu R, Zhu N, Yao HL, Lei XY, Li SX, Liao DF, and Zheng XL (2014) The nove role and underlying mechanism of Wnt5a in regulating cellular cholesterol accumulation. Clin Exp Pharmacol Physiol 41:671-678.

Qu J, Zhou J, Yi XP, Dong B, Zheng H, Miller LM, Wang X, Schneider MD, and Li F (2007) Cardiac-specific haploinsufficiency of beta-catenin attenuates cardiac hypertrophy but enhances fetal gene expression in response to aortic constriction. $J$ Mol Cell Cardiol 43:319-326.

Quackenbush KS, Bagby S, Tai WM, Messersmith WA, Schreiber A, Greene J, Kim J, Wang G, Purkey A, Pitts TM, et al. (2016) The novel tankyrase inhibitor (AZ1366) enhances irinotecan activity in tumors that exhibit elevated tankyrase and irinotecan resistance. Oncotarget 7:28273-28285.

Quasnichka H, Slater SC, Beeching CA, Boehm M, Sala-Newby GB, and George S. (2006) Regulation of smooth muscle cell proliferation by beta-catenin/T-cell factor signaling involves modulation of cyclin D1 and p21 expression. Circ Res 99:1329-1337. Rabinovitch M (2012) Molecular pathogenesis of pulmonary arterial hypertension. $J$ Clin Invest 122:4306-4313.

Rajamannan NM (2010) Mechanisms of aortic valve calcification: the LDL-densityradius theory: a translation from cell signaling to physiology. Am J Physiol Heart Circ Physiol 298:H5-H15.

Rajamannan NM (2011) The role of Lrp5/6 in cardiac valve disease: LDL-densitypressure theory. J Cell Biochem 112:2222-2229.

Rajamannan NM (2014) Myxomatous mitral valve disease bench to bedside: LDLdensity-pressure regulates Lrp5. Expert Rev Cardiovasc Ther 12:383-392.

Ramírez de Molina A, Vargas T, Molina S, Sánchez J, Martínez-Romero J, GonzálezVallinas M, Martín-Hernández R, Sánchez-Martínez R, Gómez de Cedrón M, Dávalos A, et al. (2015) The ellagic acid derivative 4,4' ${ }^{\prime}$-di-O-methylellagic acid efficiently inhibits colon cancer cell growth through a mechanism involving WNT16. J Pharmacol Exp Ther 353:433-444

Ramsey SA, Vengrenyuk Y, Menon P, Podolsky I, Feig JE, Aderem A, Fisher EA, and Gold ES (2014) Epigenome-guided analysis of the transcriptome of plaque macrophages during atherosclerosis regression reveals activation of the Wnt signaling pathway. PLoS Genet 10:e1004828.

Regitz-Zagrosek V, Oertelt-Prigione S, Prescott E, Franconi F, Gerdts E, ForystLudwig A, Maas AH, Kautzky-Willer A, Knappe-Wegner D, Kintscher U, et al.; EUGenMed Cardiovascular Clinical Study Group (2016) Gender in cardiovascular diseases: impact on clinical manifestations, management, and outcomes. Eur Heart $J$ 37:24-34.

Reis M, Czupalla CJ, Ziegler N, Devraj K, Zinke J, Seidel S, Heck R, Thom S, Macas J, Bockamp E, et al. (2012) Endothelial Wnt/ß-catenin signaling inhibits glioma angiogenesis and normalizes tumor blood vessels by inducing PDGF-B expression. $J$ Exp Med 209:1611-1627.

Rezvani M and Liew CC (2000) Role of the adenomatous polyposis coli gene product in human cardiac development and disease. J Biol Chem 275:18470-18475.

Rhodes CJ, Wharton J, Boon RA, Roexe T, Tsang H, Wojciak-Stothard B, Chakrabarti A, Howard LS, Gibbs JS, Lawrie A, et al. (2013) Reduced microRNA-150 is 
associated with poor survival in pulmonary arterial hypertension. Am J Respir Crit Care Med 187:294-302.

Richards MH, Seaton MS, Wallace J, and Al-Harthi L (2014) Porcupine is not required for the production of the majority of Wnts from primary human astrocytes and CD8+ T cells. PLoS One 9:e92159.

Rijsewijk F, Schuermann M, Wagenaar E, Parren P, Weigel D, and Nusse R (1987) The Drosophila homolog of the mouse mammary oncogene int-1 is identical to the segment polarity gene wingless. Cell 50:649-657.

Rong S, Zhao X, Jin X, Zhang Z, Chen L, Zhu Y, and Yuan W (2014) Vascular calcification in chronic kidney disease is induced by bone morphogenetic protein-2 via a mechanism involving the Wnt/ $\beta$-catenin pathway. Cell Physiol Biochem 34: 2049-2060.

Rosenberg LH, Lafitte M, Quereda V, Grant W, Chen W, Bibian M, Noguchi Y, Fallahi M, Yang C, Chang JC, et al. (2015) Therapeutic targeting of casein kinase $1 \delta$ in breast cancer. Sci Transl Med 7:318ra202.

Ruiz-Villalba A, Hoppler S, and van den Hoff MJ (2016) Wnt signaling in the heart fields: variations on a common theme. Dev Dyn 245:294-306.

Rumyantsev PP (1966) Autoradiographic study on the synthesis of DNA, RNA, and proteins in normal cardiac muscle cells and those changed by experimental injury. Folia Histochem Cytochem (Krakow) 4:397-424.

Rumyantsev PP and Marakjan VO (1968) Reactive synthesis of DNA and mitotiv division in atrial heart muscle cells following ventricle infarction. Experientia 24: 1234-1235

Ryu MJ, Cho M, Song JY, Yun YS, Choi IW, Kim DE, Park BS, and Oh S (2008) Natural derivatives of curcumin attenuate the Wnt/beta-catenin pathway through down-regulation of the transcriptional coactivator p300. Biochem Biophys Res Commun 377:1304-1308

Ryves WJ and Harwood AJ (2001) Lithium inhibits glycogen synthase kinase-3 by competition for magnesium. Biochem Biophys Res Commun 280:720-725.

Säfholm A, Leandersson K, Dejmek J, Nielsen CK, Villoutreix BO, and Andersson T (2006) A formylated hexapeptide ligand mimics the ability of Wnt-5a to impair migration of human breast epithelial cells. J Biol Chem 281:2740-2749.

Säfholm A, Tuomela J, Rosenkvist J, Dejmek J, Härkönen P, and Andersson T (2008) The Wnt-5a-derived hexapeptide Foxy- 5 inhibits breast cancer metastasis in vivo by targeting cell motility. Clin Cancer Res 14:6556-6563.

Saldanha J, Singh J, and Mahadevan D (1998) Identification of a Frizzled-like cysteine rich domain in the extracellular region of developmental receptor tyrosine kinases. Protein Sci 7:1632-1635.

Samarzija I, Sini P, Schlange T, Macdonald G, and Hynes NE (2009) Wnt3a regulates proliferation and migration of HUVEC via canonical and non-canonical Wnt signaling pathways. Biochem Biophys Res Commun 386:449-454.

Sanbe A, Gulick J, Hanks MC, Liang Q, Osinska H, and Robbins J (2003) Reengineering inducible cardiac-specific transgenesis with an attenuated myosin heavy chain promoter. Circ Res 92:609-616.

Saraswati S, Alfaro MP, Thorne CA, Atkinson J, Lee E, and Young PP (2010) Pyrvinium, a potent small molecule Wnt inhibitor, promotes wound repair and post-MI cardiac remodeling. PLoS One 5:e15521.

Sarzani R, Salvi F, Bordicchia M, Guerra F, Battistoni I, Pagliariccio G, Carbonari L, Dessì-Fulgheri P, and Rappelli A (2011) Carotid artery atherosclerosis in hypertensive patients with a functional LDL receptor-related protein 6 gene variant. Nutr Metab Cardiovasc Dis 21:150-156.

Sasaki T, Hwang H, Nguyen C, Kloner RA, and Kahn M (2013) The small molecule Wnt signaling modulator ICG-001 improves contractile function in chronically infarcted rat myocardium. PLoS One 8:e75010.

Sasaki T and Kahn M (2014) Inhibition of $\beta$-catenin/p300 interaction proximalizes mouse embryonic lung epithelium. Transl Respir Med 2:8.

Sato A, Yamamoto H, Sakane H, Koyama H, and Kikuchi A (2010a) Wnt5a regulates distinct signalling pathways by binding to Frizzled2. EMBO J 29:41-54.

Sato N, Meijer L, Skaltsounis L, Greengard P, and Brivanlou AH (2004) Maintenance of pluripotency in human and mouse embryonic stem cells through activation of Wnt signaling by a pharmacological GSK-3-specific inhibitor. Nat Med 10:55-63.

Sato N, Yamabuki T, Takano A, Koinuma J, Aragaki M, Masuda K, Ishikawa N, Kohno N, Ito H, Miyamoto M, et al. (2010b) Wnt inhibitor Dickkopf-1 as a target for passive cancer immunotherapy. Cancer Res 70:5326-5336.

Scarborough HA, Helfrich BA, Casás-Selves M, Schuller AG, Grosskurth SE, Kim J, Tan AC, Chan DC, Zhang Z, Zaberezhnyy V, et al. (2017) AZ1366: an inhibitor of Tankyrase and the canonical Wnt pathway that limits the persistence of nonsmall cell lung cancer cells following EGFR inhibition. Clin Cancer Res 23 1531-1541.

Schaale K, Neumann J, Schneider D, Ehlers S, and Reiling N (2011) Wnt signaling in macrophages: augmenting and inhibiting mycobacteria-induced inflammatory responses. Eur J Cell Biol 90:553-559.

Schleiffarth JR, Person AD, Martinsen BJ, Sukovich DJ, Neumann A, Baker CV, Lohr JL, Cornfield DN, Ekker SC, and Petryk A (2007) Wnt5a is required for cardiac outflow tract septation in mice. Pediatr Res 61:386-391.

Schneider VA and Mercola M (2001) Wnt antagonism initiates cardiogenesis in Xenopus laevis. Genes Dev 15:304-315.

Schulte G (2010) International Union of Basic and Clinical Pharmacology. LXXX. The class Frizzled receptors. Pharmacol Rev 62:632-667.

Schulte G (2015) Frizzleds and WNT/ $\beta$-catenin signaling-The black box of ligandreceptor selectivity, complex stoichiometry and activation kinetics. Eur J Pharmacol 763 (Pt B):191-195.

Schumann H, Holtz J, Zerkowski HR, and Hatzfeld M (2000) Expression of secreted frizzled related proteins 3 and 4 in human ventricular myocardium correlates with apoptosis related gene expression. Cardiovasc Res 45:720-728.

Sen-Chowdhry S, Syrris P, and McKenna WJ (2005) Genetics of right ventricular cardiomyopathy. J Cardiovasc Electrophysiol 16:927-935.

Sen M, Chamorro M, Reifert J, Corr M, and Carson DA (2001) Blockade of Wnt-5A frizzled 5 signaling inhibits rheumatoid synoviocyte activation. Arthritis Rheum 44:772-781.
Shan J, Shi DL, Wang J, and Zheng J (2005) Identification of a specific inhibitor of the dishevelled PDZ domain. Biochemistry 44:15495-15503.

Shan J, Zhang X, Bao J, Cassell R, and Zheng JJ (2012) Synthesis of potent dishevelled PDZ domain inhibitors guided by virtual screening and NMR studies. Chem Biol Drug Des 79:376-383.

Shao JS, Aly ZA, Lai CF, Cheng SL, Cai J, Huang E, Behrmann A, and Towler DA (2007) Vascular Bmp Msx2 Wnt signaling and oxidative stress in arterial calcification. Ann N Y Acad Sci 1117:40-50.

Shao JS, Cai J, and Towler DA (2006) Molecular mechanisms of vascular calcification: lessons learned from the aorta. Arterioscler Thromb Vasc Biol 26:1423-1430.

Shao JS, Cheng SL, Pingsterhaus JM, Charlton-Kachigian N, Loewy AP, and Towler DA (2005) Msx2 promotes cardiovascular calcification by activating paracrine Wnt signals. J Clin Invest 115:1210-1220.

Sharma M, Jamieson C, Lui C, and Henderson BR (2016) Distinct hydrophobic "patches" in the N- and C-tails of beta-catenin contribute to nuclear transport. Exp Cell Res 348:132-145.

Shi M, Stauffer B, Bhat R, Billiard J, Ponce-de-Leon H, Seestaller-Wehr L, Fukayama S, Mangine A, Moran R, Krishnamurthy G, et al. (2009) Identification of iminooxothiazolidines as secreted frizzled related protein-1 inhibitors. Bioorg Med Chem Lett 19:6337-6339.

Shi Y, He B, You L, and Jablons DM (2007) Roles of secreted frizzled-related proteins in cancer. Acta Pharmacol Sin 28:1499-1504.

Shultz MD, Cheung AK, Kirby CA, Firestone B, Fan J, Chen CH, Chen Z, Chin DN, Dipietro L, Fazal A, et al. (2013) Identification of NVP-TNKS656: the use of structure-efficiency relationships to generate a highly potent, selective, and orally active tankyrase inhibitor. J Med Chem 56:6495-6511.

Skaria T, Bachli E, and Schoedon G (2017) Wnt5A/Ryk signaling critically affects barrier function in human vascular endothelial cells. Cell Adhes Migr 11:24-38.

Skaria T, Burgener J, Bachli E, and Schoedon G (2016) IL-4 causes hyperpermeability of vascular endothelial cells through Wnt5A signaling. PLoS One 11: e0156002.

Sklepkiewicz P, Shiomi T, Kaur R, Sun J, Kwon S, Mercer B, Bodine P, Schermuly RT, George I, Schulze PC, et al. (2015) Loss of secreted frizzled-related protein-1 leads to deterioration of cardiac function in mice and plays a role in human cardiomyopathy. Circ Heart Fail 8:362-372.

Slater SC, Koutsouki E, Jackson CL, Bush RC, Angelini GD, Newby AC, and George SJ (2004) R-cadherin:beta-catenin complex and its association with vascular smooth muscle cell proliferation. Arterioscler Thromb Vasc Biol 24:1204-1210.

Slusarski DC, Corces VG, and Moon RT (1997) Interaction of Wnt and a Frizzled homologue triggers G-protein-linked phosphatidylinositol signalling. Nature 390: 410-413.

Smallwood PM, Williams J, Xu Q, Leahy DJ, and Nathans J (2007) Mutational analysis of Norrin-Frizzled4 recognition. $J$ Biol Chem $\mathbf{2 8 2}: 4057-4068$.

Snead AN and Insel PA (2012) Defining the cellular repertoire of GPCRs identifies a profibrotic role for the most highly expressed receptor, protease-activated receptor 1, in cardiac fibroblasts. FASEB J 26:4540-4547.

Song JL, Nigam P, Tektas SS, and Selva E (2015) microRNA regulation of Wnt signaling pathways in development and disease. Cell Signal 27:1380-1391.

Stanganello E, Hagemann AI, Mattes B, Sinner C, Meyen D, Weber S, Schug A, Raz E, and Scholpp S (2015) Filopodia-based Wnt transport during vertebrate tissue patterning. Nat Commun 6:5846.

Stanganello E and Scholpp S (2016) Role of cytonemes in Wnt transport. J Cell Sci 129:665-672.

Stenman JM, Rajagopal J, Carroll TJ, Ishibashi M, McMahon J, and McMahon AP (2008) Canonical Wnt signaling regulates organ-specific assembly and differentiation of CNS vasculature. Science 322:1247-1250.

Stoick-Cooper CL, Weidinger G, Riehle KJ, Hubbert C, Major MB, Fausto N, and Moon RT (2007) Distinct Wnt signaling pathways have opposing roles in appendage regeneration. Development 134:479-489.

Sun LY, Bie ZD, Zhang CH, Li H, Li LD, and Yang J (2016) MiR-154 directly suppresses DKK2 to activate Wnt signaling pathway and enhance activation of cardiac fibroblasts. Cell Biol Int 40:1271-1279.

Sun Y, Liang X, Najafi N, Cass M, Lin L, Cai CL, Chen J, and Evans SM (2007) Islet 1 is expressed in distinct cardiovascular lineages, including pacemaker and coronary vascular cells. Dev Biol 304:286-296.

Sutherland C, Leighton IA, and Cohen P (1993) Inactivation of glycogen synthase kinase- 3 beta by phosphorylation: new kinase connections in insulin and growthfactor signalling. Biochem J 296:15-19.

Swope D, Cheng L, Gao E, Li J, and Radice GL (2012) Loss of cadherin-binding proteins $\beta$-catenin and plakoglobin in the heart leads to gap junction remodeling and arrhythmogenesis. Mol Cell Biol 32:1056-1067.

Sylva M, van den Hoff MJ, and Moorman AF (2014) Development of the human heart. Am J Med Genet A 164A:1347-1371.

Tago K, Nakamura T, Nishita M, Hyodo J, Nagai S, Murata Y, Adachi S, Ohwada S, Morishita Y, Shibuya H, et al. (2000) Inhibition of Wnt signaling by ICAT, a novel beta-catenin-interacting protein. Genes Dev 14:1741-1749.

Takada K, Zhu D, Bird GH, Sukhdeo K, Zhao JJ, Mani M, Lemieux M, Carrasco DE, Ryan J, Horst D, et al. (2012) Targeted disruption of the BCL9/ß-catenin complex inhibits oncogenic Wnt signaling. Sci Transl Med 4:148ra117.

Takahashi-Yanaga F (2013) Activator or inhibitor? GSK-3 as a new drug target. Biochem Pharmacol 86:191-199.

Takahashi J, Orcholski M, Yuan K, and de Jesus Perez V (2016) PDGF-dependent $\beta$-catenin activation is associated with abnormal pulmonary artery smooth muscle cell proliferation in pulmonary arterial hypertension. FEBS Lett 590:101-109.

Takeuchi S, Takeda K, Oishi I, Nomi M, Ikeya M, Itoh K, Tamura S, Ueda T, Hatta T, Otani H, et al. (2000) Mouse Ror2 receptor tyrosine kinase is required for the heart development and limb formation. Genes Cells 5:71-78.

Tamai K, Semenov M, Kato Y, Spokony R, Liu C, Katsuyama Y, Hess F, SaintJeannet JP, and He X (2000) LDL-receptor-related proteins in Wnt signal transduction. Nature 407:530-535. 
Tao J, Wang YT, Abudoukelimu M, Yang YN, Li XM, Xie X, Chen BD, Liu F, He CH, $\mathrm{Li} \mathrm{HY}$, et al. (2016) Association of genetic variations in the Wnt signaling pathway genes with myocardial infarction susceptibility in Chinese Han population. Oncotarget 7:52740-52750.

Tateishi A, Matsushita M, Asai T, Masuda Z, Kuriyama M, Kanki K, Ishino K, Kawada M, and Sano S (2010) Effect of inhibition of glycogen synthase kinase-3 on cardiac hypertrophy during acute pressure overload. Gen Thorac Cardiovasc Surg 58:265-270.

Teiten MH, Gaascht F, Cronauer M, Henry E, Dicato M, and Diederich M (2011) Anti-proliferative potential of curcumin in androgen-dependent prostate cance cells occurs through modulation of the Wingless signaling pathway. Int J Oncol 38: 603-611.

ter Horst P, Smits JF, and Blankesteijn WM (2012) The Wnt/Frizzled pathway as a therapeutic target for cardiac hypertrophy: where do we stand? Acta Physiol $(\mathrm{Oxf})$ 204:110-117.

Terami H, Hidaka K, Katsumata T, Iio A, and Morisaki T (2004) Wnt11 facilitates embryonic stem cell differentiation to Nkx2.5-positive cardiomyocytes. Biochem Biophys Res Commun 325:968-975.

Thorne CA, Hanson AJ, Schneider J, Tahinci E, Orton D, Cselenyi CS, Jernigan KK, Meyers KC, Hang BI, Waterson AG, et al. (2010) Small-molecule inhibition of Wnt signaling through activation of casein kinase 1 $\alpha$. Nat Chem Biol 6:829-836.

Tian W, Han X, Yan M, Xu Y, Duggineni S, Lin N, Luo G, Li YM, Han X, Huang Z, et al. (2012) Structure-based discovery of a novel inhibitor targeting the $\beta$-catenin Tcf4 interaction. Biochemistry 51:724-731.

Tian X, Jee WS, Li X, Paszty C, and Ke HZ (2011) Sclerostin antibody increases bone mass by stimulating bone formation and inhibiting bone resorption in a hindlimbimmobilization rat model. Bone 48:197-201.

Tian Y, Yuan L, Goss AM, Wang T, Yang J, Lepore JJ, Zhou D, Schwartz RJ, Patel V, Cohen ED, et al. (2010) Characterization and in vivo pharmacological rescue of a Wnt2-Gata6 pathway required for cardiac inflow tract development. Dev Cell 18 275-287.

Tinsley HN, Gary BD, Thaiparambil J, Li N, Lu W, Li Y, Maxuitenko YY, Keeton AB, and Piazza GA (2010) Colon tumor cell growth-inhibitory activity of sulindac sulfide and other nonsteroidal anti-inflammatory drugs is associated with phosphodiesterase 5 inhibition. Cancer Prev Res (Phila) 3:1303-1313.

Tiwari SK, Agarwal S, Tripathi A, and Chaturvedi RK (2016) Bisphenol-A mediated inhibition of hippocampal neurogenesis attenuated by curcumin via canonical Wnt pathway. Mol Neurobiol 53:3010-3029.

Toomes C, Bottomley HM, Jackson RM, Towns KV, Scott S, Mackey DA, Craig JE, Jiang L, Yang Z, Trembath R, et al. (2004) Mutations in LRP5 or FZD4 underlie the common familial exudative vitreoretinopathy locus on chromosome 11q. Am J Hum Genet 74:721-730.

Tsaousi A, Mill C, and George SJ (2011a) The Wnt pathways in vascular disease: lessons from vascular development. Curr Opin Lipidol 22:350-357.

Tsaousi A, Williams H, Lyon CA, Taylor V, Swain A, Johnson JL, and George SJ (2011b) Wnt4/ß-catenin signaling induces VSMC proliferation and is associated with intimal thickening. Circ Res 108:427-436.

Tseng AS, Engel FB, and Keating MT (2006) The GSK-3 inhibitor BIO promotes proliferation in mammalian cardiomyocytes. Chem Biol 13:957-963

Tsukamoto AS, Grosschedl R, Guzman RC, Parslow T, and Varmus HE (1988) Expression of the int-1 gene in transgenic mice is associated with mammary gland hyperplasia and adenocarcinomas in male and female mice. Cell 55:619-625.

Tzeng HE, Yang L, Chen K, Wang Y, Liu YR, Pan SL, Gaur S, Hu S, and Yen Y (2015) The pan-PI3K inhibitor GDC-0941 activates canonical WNT signaling to confer resistance in TNBC cells: resistance reversal with WNT inhibitor. Oncotarget 6 11061-11073.

Ueland T, Otterdal K, Lekva T, Halvorsen B, Gabrielsen A, Sandberg WJ Paulsson-Berne G, Pedersen TM, Folkersen L, Gullestad L, et al. (2009) Dickkopf-1 enhances inflammatory interaction between platelets and endothelial cells and shows increased expression in atherosclerosis. Arterioscler Thromb Vasc Biol 29:1228-1234.

Ueno S, Weidinger G, Osugi T, Kohn AD, Golob JL, Pabon L, Reinecke H, Moon RT, and Murry CE (2007) Biphasic role for Wnt/beta-catenin signaling in cardiac specification in zebrafish and embryonic stem cells. Proc Natl Acad Sci USA 104 9685-9690.

Uglow EB, Slater S, Sala-Newby GB, Aguilera-Garcia CM, Angelini GD, Newby AC, and George SJ (2003) Dismantling of cadherin-mediated cell-cell contacts modulates smooth muscle cell proliferation. Circ Res 92:1314-1321.

Uitterdijk A, Hermans KC, de Wijs-Meijler DP, Daskalopoulos EP, Reiss IK, Duncker DJ, Matthijs Blankesteijn W, and Merkus D (2016) UM206, a selective Frizzled antagonist, attenuates adverse remodeling after myocardial infarction in swine. Lab Invest 96:168-176.

Vaduganathan M, Greene SJ, Ambrosy AP, Gheorghiade M, and Butler J (2013) The disconnect between phase II and phase III trials of drugs for heart failure. Nat Rev Cardiol 10:85-97.

Valenta T, Hausmann G, and Basler K (2012) The many faces and functions of ß-catenin. EMBO J 31:2714-2736.

van Amerongen R and Nusse R (2009) Towards an integrated view of Wnt signaling in development. Development 136:3205-3214.

van de Schans VA, van den Borne SW, Strzelecka AE, Janssen BJ, van der Velden JL, Langen RC, Wynshaw-Boris A, Smits JF, and Blankesteijn WM (2007) Interruption of Wnt signaling attenuates the onset of pressure overload-induced cardiac hypertrophy. Hypertension 49:473-480.

van de Wetering M, Cavallo R, Dooijes D, van Beest M, van Es J, Loureiro J, Ypma A Hursh D, Jones T, Bejsovec A, et al. (1997) Armadillo coactivates transcription driven by the product of the Drosophila segment polarity gene dTCF. Cell 88 789-799

van den Borne SW, Diez J, Blankesteijn WM, Verjans J, Hofstra L, and Narula J (2010) Myocardial remodeling after infarction: the role of myofibroblasts. Nat Rev Cardiol 7:30-37. van den Bosch $\mathrm{MH}$, Gleissl TA, Blom $\mathrm{AB}$, van den Berg WB, van Lent PL, and van der Kraan PM (2016) Wnts talking with the TGF- $\beta$ superfamily: WISPers about modulation of osteoarthritis. Rheumatology (Oxford) 55:1536-1547.

van den Hoff MJ and Moorman AF (2000) Cardiac neural crest: the holy grail of cardiac abnormalities? Cardiovasc Res 47:212-216.

van Dinther M, Zhang J, Weidauer SE, Boschert V, Muth EM, Knappik A, de Gorter DJ, van Kasteren PB, Frisch C, Mueller TD, et al. (2013) Anti-Sclerostin antibody inhibits internalization of Sclerostin and Sclerostin-mediated antagonism of Wnt/ LRP6 signaling. PLoS One 8:e62295.

Vanhollebeke B, Stone OA, Bostaille N, Cho C, Zhou Y, Maquet E, Gauquier A Cabochette P, Fukuhara S, Mochizuki N, et al. (2015) Tip cell-specific requirement for an atypical Gpr124- and Reck-dependent Wnt/ $\beta$-catenin pathway during brain angiogenesis. eLife 4:e6489.

Venerando A, Girardi C, Ruzzene M, and Pinna LA (2013) Pyrvinium pamoate does not activate protein kinase CK1, but promotes Akt/PKB down-regulation and GSK3 activation. Biochem $J$ 452:131-137.

Vermij SH, Abriel H, and van Veen TA (2017) Refining the molecular organization of the cardiac intercalated disc. Cardiovasc Res 113:259-275.

Vervloet M and Cozzolino M (2017) Vascular calcification in chronic kidney disease: different bricks in the wall? Kidney Int 91:808-817.

Vikram A, Kim YR, Kumar S, Naqvi A, Hoffman TA, Kumar A, Miller FJ Jr, Kim CS, and Irani K (2014) Canonical Wnt signaling induces vascular endothelial dysfunction via p66Shc-regulated reactive oxygen species. Arterioscler Thromb Vasc Biol 34:2301-2309.

Visweswaran M, Pohl S, Arfuso F, Newsholme P, Dilley R, Pervaiz S, and Dharmarajan A (2015) Multi-lineage differentiation of mesenchymal stem cells To Wnt, or not Wnt. Int J Biochem Cell Biol 68:139-147.

Waaler J, Machon O, Tumova L, Dinh H, Korinek V, Wilson SR, Paulsen JE, Pedersen NM, Eide TJ, Machonova O, et al. (2012) A novel tankyrase inhibitor decreases canonical Wnt signaling in colon carcinoma cells and reduces tumor growth in conditional APC mutant mice. Cancer Res 72:2822-2832

Waaler J, Machon O, von Kries JP, Wilson SR, Lundenes E, Wedlich D, Gradl D, Paulsen JE, Machonova O, Dembinski JL, et al. (2011) Novel synthetic antagonists of canonical Wnt signaling inhibit colorectal cancer cell growth. Cancer Res 71:197-205.

Wang C, Zhu H, Sun Z, Xiang Z, Ge Y, Ni C, Luo Z, Qian W, and Han X (2014a) Inhibition of Wnt/ $\beta$-catenin signaling promotes epithelial differentiation of mesenchymal stem cells and repairs bleomycin-induced lung injury. Am J Physiol Cell Physiol 307:C234-C244.

Wang H, Charles PC, Wu Y, Ren R, Pi X, Moser M, Barshishat-Kupper M, Rubin JS, Perou C, Bautch V, et al. (2006) Gene expression profile signatures indicate a role for Wnt signaling in endothelial commitment from embryonic stem cells. Circ Res 98:1331-1339.

Wang H, Gilner JB, Bautch VL, Wang DZ, Wainwright BJ, Kirby SL, and Patterson C (2007) Wnt2 coordinates the commitment of mesoderm to hematopoietic, endothelial, and cardiac lineages in embryoid bodies. J Biol Chem 282:782-791.

Wang J, Panáková D, Kikuchi K, Holdway JE, Gemberling M, Burris JS, Singh SP, Dickson AL, Lin YF, Sabeh MK, et al. (2011a) The regenerative capacity of zebrafish reverses cardiac failure caused by genetic cardiomyocyte depletion. $D e-$ velopment 138:3421-3430.

Wang L, Hu XB, Zhang W, Wu LD, Liu YS, Hu B, Bi CL, Chen YF, Liu XX, Ge C, et al. (2013a) Dickkopf-1 as a novel predictor is associated with risk stratification by GRACE risk scores for predictive value in patients with acute coronary syndrome: a retrospective research. PLoS One 8:e54731.

Wang W, Liu H, Wang S, Hao X, and Li L (2011b) A diterpenoid derivative 15 -oxospiramilactone inhibits Wnt/ $\beta$-catenin signaling and colon cancer cell tumorigenesis. Cell Res 21:730-740.

Wang X, Adhikari N, Li Q, and Hall JL (2004) LDL receptor-related protein LRP6 regulates proliferation and survival through the Wnt cascade in vascular smooth muscle cells. Am J Physiol Heart Circ Physiol 287:H2376-H2383.

Wang X, Moon J, Dodge ME, Pan X, Zhang L, Hanson JM, Tuladhar R, Ma Z, Shi H, Williams NS, et al. (2013b) The development of highly potent inhibitors for porcupine. J Med Chem 56:2700-2704.

Wang X, Xiao Y, Mou Y, Zhao Y, Blankesteijn WM, and Hall JL (2002) A role for the beta-catenin/T-cell factor signaling cascade in vascular remodeling. Circ Res $\mathbf{9 0}$ 340-347.

Wang Y, Chang H, Rattner A, and Nathans J (2016a) Frizzled receptors in development and disease. Curr Top Dev Biol 117:113-139.

Wang Y, Huso D, Cahill H, Ryugo D, and Nathans J (2001) Progressive cerebellar, auditory, and esophageal dysfunction caused by targeted disruption of the frizzled4 gene. $J$ Neurosci 21:4761-4771.

Wang Y, Li YP, Paulson C, Shao JZ, Zhang X, Wu M, and Chen W (2014b) Wnt and the Wnt signaling pathway in bone development and disease. Front Biosci (Landmark Ed) 19:379-407.

Wang Y, Liu J, Cui J, Sun M, Du W, Chen T, Ming X, Zhang L, Tian J, Li J, et al. (2016b) MiR218 modulates Wnt signaling in mouse cardiac stem cells by promoting proliferation and inhibiting differentiation through a positive feedback loop. Sci Rep 6:20968.

Wang Y, Rattner A, Zhou Y, Williams J, Smallwood PM, and Nathans J (2012) Norrin/Frizzled4 signaling in retinal vascular development and blood brain barrier plasticity. Cell 151:1332-1344.

Wang Y, Sang A, Zhu M, Zhang G, Guan H, Ji M, and Chen H (2016c) Tissue factor induces VEGF expression via activation of the Wnt/ $\beta$-catenin signaling pathway in ARPE-19 cells. Mol Vis 22:886-897.

Warden SM, Andreoli CM, and Mukai S (2007) The Wnt signaling pathway in familial exudative vitreoretinopathy and Norrie disease. Semin Ophthalmol 22: 211-217.

Webb IG, Nishino Y, Clark JE, Murdoch C, Walker SJ, Makowski MR, Botnar RM, Redwood SR, Shah AM, and Marber MS (2010) Constitutive glycogen synthase kinase-3alpha/beta activity protects against chronic beta-adrenergic remodelling of the heart. Cardiovasc Res 87:494-503. 
Wehrli M, Dougan ST, Caldwell K, O'Keefe L, Schwartz S, Vaizel-Ohayon D, Schejter E, Tomlinson A, and DiNardo S (2000) arrow encodes an LDL-receptor-related protein essential for Wingless signalling. Nature 407:527-530.

Wen J, Liu J, Song G, Liu L, Tang B, and Li Z (2010) Effects of 6-bromoindirubin-3'oxime on the maintenance of pluripotency of porcine embryonic germ cells in combination with stem cell factor, leukemia inhibitory factor and fibroblast growth factor. Reproduction 139:1039-1046.

West JD, Austin ED, Gaskill C, Marriott S, Baskir R, Bilousova G, Jean JC, Hemnes AR, Menon S, Bloodworth NC, et al. (2014) Identification of a common Wntassociated genetic signature across multiple cell types in pulmonary arterial hypertension. Am J Physiol Cell Physiol 307:C415-C430.

Wexler EM, Geschwind DH, and Palmer TD (2008) Lithium regulates adult hippocampal progenitor development through canonical Wnt pathway activation. Mol Psychiatry 13:285-292.

Willems E, Spiering S, Davidovics H, Lanier M, Xia Z, Dawson M, Cashman J, and Mercola M (2011) Small-molecule inhibitors of the Wnt pathway potently promote cardiomyocytes from human embryonic stem cell-derived mesoderm. Circ Res 109:360-364.

Willert K, Brown JD, Danenberg E, Duncan AW, Weissman IL, Reya T, Yates JR III, and Nusse R (2003) Wnt proteins are lipid-modified and can act as stem cell growth factors. Nature 423:448-452.

Willert K and Nusse R (2012) Wnt proteins. Cold Spring Harb Perspect Biol 4: a007864.

Wisniewski JA, Yin J, Teuscher KB, Zhang M, and Ji H (2016) Structure-based design of 1,4-dibenzoylpiperazines as $\beta$-catenin/B-cell lymphoma 9 protein-protein interaction inhibitors. ACS Med Chem Lett 7:508-513.

Witman N, Murtuza B, Davis B, Arner A, and Morrison JI (2011) Recapitulation of developmental cardiogenesis governs the morphological and functional regeneration of adult newt hearts following injury. Dev Biol 354:67-76.

Wo D, Peng J, Ren DN, Qiu L, Chen J, Zhu Y, Yan Y, Yan H, Wu J, Ma E, et al. (2016) Opposing roles of Wnt inhibitors IGFBP-4 and Dkk1 in cardiac ischemia by differential targeting of LRP5/6 and $\beta$-catenin. Circulation 134:1991-2007.

Wolf J, Palmby TR, Gavard J, Williams BO, and Gutkind JS (2008) Multiple PPPS/ TP motifs act in a combinatorial fashion to transduce Wnt signaling through LRP6. FEBS Lett 582:255-261.

Wong HC, Bourdelas A, Krauss A, Lee HJ, Shao Y, Wu D, Mlodzik M, Shi DL, and Zheng J (2003) Direct binding of the PDZ domain of dishevelled to a conserved internal sequence in the C-terminal region of frizzled. Mol Cell 12:1251-1260.

Woulfe KC, Gao E, Lal H, Harris D, Fan Q, Vagnozzi R, DeCaul M, Shang X, Patel S, Woodgett JR, et al. (2010) Glycogen synthase kinase-3beta regulates postmyocardial infarction remodeling and stress-induced cardiomyocyte proliferation in vivo. Circ Res 106:1635-1645.

Wu D, Talbot CC Jr, Liu Q, Jing ZC, Damico RL, Tuder R, Barnes KC, Hassoun PM, and Gao L (2016a) Identifying microRNAs targeting Wnt/ $\beta$-catenin pathway in end-stage idiopathic pulmonary arterial hypertension. J Mol Med (Berl) 94 $875-885$.

Wu J, Roman AC, Carvajal-Gonzalez JM, and Mlodzik M (2013) Wg and Wnt4 provide long-range directional input to planar cell polarity orientation in Drosophila. Nat Cell Biol 15:1045-1055.

Wu SM (2008) Mesp1 at the heart of mesoderm lineage specification. Cell Stem Cell $\mathbf{3}$ $1-2$.

Wu X, Luo F, Li J, Zhong X, and Liu K (2016b) Tankyrase 1 inhibitior XAV939 increases chemosensitivity in colon cancer cell lines via inhibition of the Wnt signaling pathway. Int $J$ Oncol 48:1333-1340.

Wynshaw-Boris A (2012) Dishevelled: in vivo roles of a multifunctional gene family during development. Curr Top Dev Biol 101:213-235.

Xiao Y, Leach J, Wang J, and Martin JF (2016) Hippo/Yap signaling in cardiac development and regeneration. Curr Treat Options Cardiovasc Med 18:38.

Xin H, Xin F, Zhou S, and Guan S (2013) The Wnt5a/Ror2 pathway is associated with determination of the differentiation fate of bone marrow mesenchymal stem cells in vascular calcification. Int $J$ Mol Med 31:583-588.

Xu Q, Wang Y, Dabdoub A, Smallwood PM, Williams J, Woods C, Kelley MW, Jiang L, Tasman W, Zhang K, et al. (2004) Vascular development in the retina and inner ear: control by Norrin and Frizzled-4, a high-affinity ligand-receptor pair. Cell 116: $883-895$

Xu YK and Nusse R (1998) The Frizzled CRD domain is conserved in diverse proteins including several receptor tyrosine kinases. Curr Biol 8:R405-R406.

Xu Z, Xu X, O’Laoi R, Ma H, Zheng J, Chen S, Luo L, Hu Z, He S, Li J, et al. (2016 Design, synthesis, and evaluation of novel porcupine inhibitors featuring a fused 3-ring system based on the 'reversed' amide scaffold. Bioorg Med Chem 24: $5861-5872$

Yan C, Jamaluddin MS, Aggarwal B, Myers J, and Boyd DD (2005) Gene expression profiling identifies activating transcription factor 3 as a novel contributor to the proapoptotic effect of curcumin. Mol Cancer Ther 4:233-241.

Yang DH, Yoon JY, Lee SH, Bryja V, Andersson ER, Arenas E, Kwon YG, and Choi $\mathrm{KY}$ (2009) Wnt5a is required for endothelial differentiation of embryonic stem cells and vascularization via pathways involving both Wnt/beta-catenin and protein kinase Calpha. Circ Res 104:372-379.

Yang Y and Mlodzik M (2015) Wnt-Frizzled/planar cell polarity signaling: cellular orientation by facing the wind (Wnt). Annu Rev Cell Dev Biol 31:623-646.

Yao YY, Yin H, Shen B, Smith RS Jr, Liu Y, Gao L, Chao L, and Chao J (2008) Tissue kallikrein promotes neovascularization and improves cardiac function by the Aktglycogen synthase kinase-3beta pathway. Cardiovasc Res 80:354-364.

Ye B, Ge Y, Perens G, Hong L, Xu H, Fishbein MC, and Li F (2013) Canonical Wnt/ $\beta$-catenin signaling in epicardial fibrosis of failed pediatric heart allografts with diastolic dysfunction. Cardiovasc Pathol 22:54-57.

Ye Q, Yao G, Zhang M, Guo G, Hu Y, Jiang J, Cheng L, Shi J, Li H, Zhang Y, et al. (2015) A novel ent-kaurane diterpenoid executes antitumor function in colorectal cancer cells by inhibiting Wnt/ $\beta$-catenin signaling. Carcinogenesis 36 318-326.
Ye S, Tan L, Yang R, Fang B, Qu S, Schulze EN, Song H, Ying Q, and Li P (2012) Pleiotropy of glycogen synthase kinase-3 inhibition by CHIR99021 promotes selfrenewal of embryonic stem cells from refractory mouse strains. PLoS One 7: e35892.

Ye X, Wang Y, Cahill H, Yu M, Badea TC, Smallwood PM, Peachey NS, and Nathans J (2009) Norrin, frizzled-4, and Lrp5 signaling in endothelial cells controls a genetic program for retinal vascularization. Cell 139:285-298.

Ye X, Wang Y, and Nathans J (2010) The Norrin/Frizzled4 signaling pathway in retinal vascular development and disease. Trends Mol Med 16:417-425.

Yu H, Smallwood PM, Wang Y, Vidaltamayo R, Reed R, and Nathans J (2010) Frizzled 1 and frizzled 2 genes function in palate, ventricular septum and neural tube closure: general implications for tissue fusion processes. Development 137 3707-3717.

Yu H, Ye X, Guo N, and Nathans J (2012) Frizzled 2 and frizzled 7 function redundantly in convergent extension and closure of the ventricular septum and palate: evidence for a network of interacting genes. Development 139: 4383-4394.

Yuan K, Orcholski ME, Panaroni C, Shuffle EM, Huang NF, Jiang X, Tian W, Vladar EK, Wang L, Nicolls MR, et al. (2015) Activation of the Wnt/planar cell polarity pathway is required for pericyte recruitment during pulmonary angiogenesis. $A m J$ Pathol 185:69-84.

Zamora M, Männer J, and Ruiz-Lozano P (2007) Epicardium-derived progenitor cells require beta-catenin for coronary artery formation. Proc Natl Acad Sci USA 104 18109-18114.

Zelarayán LC, Noack C, Sekkali B, Kmecova J, Gehrke C, Renger A, Zafiriou MP, van der Nagel R, Dietz R, de Windt LJ, et al. (2008) Beta-Catenin downregulation attenuates ischemic cardiac remodeling through enhanced resident precursor cell differentiation. Proc Natl Acad Sci USA 105:19762-19767.

Zerlin M, Julius MA, and Kitajewski J (2008) Wnt/Frizzled signaling in angiogenesis. Angiogenesis 11:63-69.

Zhai P, Gao S, Holle E, Yu X, Yatani A, Wagner T, and Sadoshima J (2007) Glycogen synthase kinase-3alpha reduces cardiac growth and pressure overload-induced cardiac hypertrophy by inhibition of extracellular signal-regulated kinases. J Biol Chem 282:33181-33191.

Zhang CG, Jia ZQ, Li BH, Zhang H, Liu YN, Chen P, Ma KT, and Zhou CY (2009a) beta-Catenin/TCF/LEF1 can directly regulate phenylephrine-induced cell hypertrophy and Anf transcription in cardiomyocytes. Biochem Biophys Res Commun 390:258-262.

Zhang K, Guo X, Zhao W, Niu G, Mo X, and Fu Q (2015a) Application of Wnt pathway inhibitor delivering Scaffold for inhibiting fibrosis in urethra strictures: in vitro and in vivo study. Int $J$ Mol Sci 16:27659-27676.

Zhang K, Harada Y, Wei X, Shukla D, Rajendran A, Tawansy K, Bedell M, Lim S, Shaw PX, He X, et al. (2011) An essential role of the cysteine-rich domain of FZD4 in Norrin/Wnt signaling and familial exudative vitreoretinopathy. $J$ Biol Chem 286:10210-10215.

Zhang K, Zhu S, Liu Y, Dong X, Shi Z, Zhang A, Liu C, Chen L, Wei J, Pu P, et al. (2015b) ICAT inhibits glioblastoma cell proliferation by suppressing Wnt/ $\beta$-catenin activity. Cancer Lett 357:404-411.

Zhang LL, Liu JJ, Liu F, Liu WH, Wang YS, Zhu B, and Yu B (2012) MiR-499 induces cardiac differentiation of rat mesenchymal stem cells through wnt/ $\beta$-catenin signaling pathway. Biochem Biophys Res Commun 420:875-881.

Zhang M, Hagenmueller M, Riffel JH, Kreusser MM, Bernhold E, Fan J, Katus HA Backs J, and Hardt SE (2015c) Calcium/calmodulin-dependent protein kinase II couples Wnt signaling with histone deacetylase 4 and mediates dishevelledinduced cardiomyopathy. Hypertension 65:335-344.

Zhang Q, Gao M, Luo G, Han X, Bao W, Cheng Y, Tian W, Yan M, Yang G, and An J (2016a) Enhancement of radiation sensitivity in lung cancer cells by a novel small molecule inhibitor that targets the $\beta$-Catenin/Tcf4 interaction. PLoS One 11: e0152407.

Zhang X, Gaspard JP, and Chung DC (2001) Regulation of vascular endothelial growth factor by the Wnt and K-ras pathways in colonic neoplasia. Cancer Res 61 : $6050-6054$

Zhang X, MacDonald BT, Gao H, Shamashkin M, Coyle AJ, Martinez RV, and He X (2016b) Characterization of tiki, a new family of Wnt-specific metalloproteases. $J$ Biol Chem 291:2435-2443.

Zhang Y, Appleton BA, Wiesmann C, Lau T, Costa M, Hannoush RN, and Sidhu SS (2009b) Inhibition of Wnt signaling by dishevelled PDZ peptides. Nat Chem Biol 5: $217-219$

Zhang Y, Liu Y, Zhu XH, Zhang XD, Jiang DS, Bian ZY, Zhang XF, Chen K, Wei X, Gao L, et al. (2014) Dickkopf-3 attenuates pressure overload-induced cardiac remodelling. Cardiovasc Res 102:35-45.

Zhang Z, Nör F, Oh M, Cucco C, Shi S, and Nör JE (2016c) Wnt/ß-catenin signaling determines the vasculogenic fate of postnatal mesenchymal stem cells. Stem Cells 34:1576-1587.

Zheng Q, Chen P, Xu Z, Li F, and Yi XP (2013) Expression and redistribution of $\beta$-catenin in the cardiac myocytes of left ventricle of spontaneously hypertensive rat. J Mol Histol 44:565-573.

Zheng R, Deng Q, Liu Y, and Zhao P (2017) Curcumin inhibits gastric carcinoma cell growth and induces apoptosis by suppressing the Wnt/ $\beta$-catenin signaling pathway. Med Sci Monit 23:163-171.

Zhong X, Zhao E, Tang C, Zhang W, Tan J, Dong Z, Ding HF, and Cui H (2016) Antibiotic drug tigecycline reduces neuroblastoma cells proliferation by inhibiting Akt activation in vitro and in vivo. Tumour Biol 37:7615-7623.

Zhou J, Ahmad F, Parikh S, Hoffman NE, Rajan S, Verma VK, Song J, Yuan A Shanmughapriya S, Guo Y, et al. (2016) Loss of adult cardiac myocyte GSK-3 leads to mitotic catastrophe resulting in fatal dilated cardiomyopathy. Circ Res 118: 1208-1222.

Zhou L, Li Y, Hao S, Zhou D, Tan RJ, Nie J, Hou FF, Kahn M, and Liu Y (2015) Multiple genes of the renin-angiotensin system are novel targets of Wnt/ $\beta$-catenin signaling. J Am Soc Nephrol 26:107-120. 
Zhou MW, Yin WT, Jiang RH, Lee JH, Kim CD, Lee JH, Zhu MJ, and Yoon TJ (2017) Inhibition of collagen synthesis by IWR-1 in normal and keloid-derived skin fibroblasts. Life Sci 173:86-93.

Zhou W, Lin L, Majumdar A, Li X, Zhang X, Liu W, Etheridge L, Shi Y, Martin J, Van de Ven W, et al. (2007) Modulation of morphogenesis by noncanonical Wnt signaling requires ATF/CREB family-mediated transcriptional activation of TGFbeta2. Nat Genet 39:1225-1234.

Zhou Y, Wang Y, Tischfield M, Williams J, Smallwood PM, Rattner A, Taketo MM, and Nathans J (2014) Canonical WNT signaling components in vascular development and barrier formation. J Clin Invest 124:3825-3846.
Zhu X, Wang W, Zhang X, Bai J, Chen G, Li L, and Li M (2015) All-trans retinoic acid-induced deficiency of the Wnt/ $\beta$-catenin pathway enhances hepatic carcinoma stem cell differentiation. PLoS One 10:e0143255.

Zi X, Guo Y, Simoneau AR, Hope C, Xie J, Holcombe RF, and Hoang BH (2005) Expression of Frzb/secreted Frizzled-related protein 3, a secreted Wnt antagonist, in human androgen-independent prostate cancer PC-3 cells suppresses tumor growth and cellular invasiveness. Cancer Res 65:9762-9770.

Zuercher J, Fritzsche M, Feil S, Mohn L, and Berger W (2012) Norrin stimulates cell proliferation in the superficial retinal vascular plexus and is pivotal for the recruitment of mural cells. Hum Mol Genet 21:2619-2630. 